\title{
Chronic low back pain : assessment and treatment from a behavioral rehabilitation perspective
}

Citation for published version (APA):

Vlaeyen, J. W. S. (1991). Chronic low back pain : assessment and treatment from a behavioral rehabilitation perspective. [Doctoral Thesis, Maastricht University]. Swets \& Zeitlinger. https://doi.org/10.26481/dis.19910321jv

Document status and date:

Published: 01/01/1991

DOI:

10.26481/dis.19910321jv

Document Version:

Publisher's PDF, also known as Version of record

\section{Please check the document version of this publication:}

- A submitted manuscript is the version of the article upon submission and before peer-review. There can be important differences between the submitted version and the official published version of record.

People interested in the research are advised to contact the author for the final version of the publication, or visit the DOI to the publisher's website.

- The final author version and the galley proof are versions of the publication after peer review.

- The final published version features the final layout of the paper including the volume, issue and page numbers.

Link to publication

\footnotetext{
General rights rights.

- You may freely distribute the URL identifying the publication in the public portal. please follow below link for the End User Agreement:

www.umlib.nl/taverne-license

Take down policy

If you believe that this document breaches copyright please contact us at:

repository@maastrichtuniversity.nl

providing details and we will investigate your claim.
}

Copyright and moral rights for the publications made accessible in the public portal are retained by the authors and/or other copyright owners and it is a condition of accessing publications that users recognise and abide by the legal requirements associated with these

- Users may download and print one copy of any publication from the public portal for the purpose of private study or research.

- You may not further distribute the material or use it for any profit-making activity or commercial gain

If the publication is distributed under the terms of Article $25 \mathrm{fa}$ of the Dutch Copyright Act, indicated by the "Taverne" license above, 
Cip-gegevens Koninklijk Bibliotheek, Den Haag

Vlaeyen, Johannes Wolfgang Silvain

Chronic low back pain : assessment and treatment from a behavioral rehabilitation perspective / Johannes Wolfgang Silvain Vlaeyen. - Ansterdam [etc.] :

Swets \& Zeitlinger- (IRV Series in rehabilitation research; 1) proefschrift Maastricht -Met llt. opg. - Met samenvatting in het Nederlands.

ISBN $90-265-1172-8$

NUGI 742

Trefw.: chronische rugklachten ; revalidatie

Omslagontwerp: Rob Molthoff

Druk omslag: Casparie, IJsselstein

Druk: Offsetdrukkerij Kanters B.V., Alblasserdam

C 1991 Swets \& Zeitlinger B.V., Amsterdam/Lisse

All rights reserved. No part of this publication nay be reproduced, stored in a retrieval system, or transwitted, in awy form or by any means, electromic, mechanical, photocopying, recording, or otherwise, without the prior written permission of the publisher.

ISBN 9026511728

NUGI 742 


\title{
CHRONIC LOW BACK PAIN
}

\section{ASSESSMENT AND TREATMENT FROM A BEHAVIORAL REHABILITATION PERSPECTIVE}

\author{
PROEFSCHRIFT
}

ter verkrijging van de graad van

Doctor aan de Rijksuniversiteit Limburg te Maastricht op gezag van de Rector Magnificus,

Prof. Mr. M.J. Cohen, volgens het besluit van het College van Dekanen, in het openbaar te verdedigen op donderdag, 21 maart 1991 om 16.00 uur

door

Johannes Wolfgang Silvain Vlaeyen

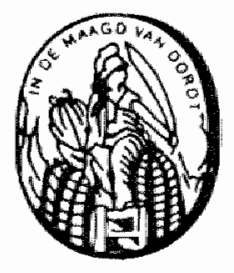

SWETS \& ZEITLINGER B.V. AMSTERDAM / LISSE 


\section{Promotor:}

Prof. dr. J.J.C.B. Bremer

\section{Co-promotores:}

Dr. N.H. Groenman

Dr. ing. J.A. Schuerman

Beoordelingscommissie:

Prof. dr. M.A. van den Hout (voorzitter)

Prof. drs. W.H. Eisma (Rijksuniversiteit Groningen)

Prof. dr. W.E. Fordyce (University of Washington, Seattle, USA) Prof. dr. J.M.J.P. van der Linden

Prof. dr. M.E. Sluijter 
Aan:

joris en margriet, nicole 



\section{CONTENTS}

PART I: CHRONIC PAIN AND THE BEHAVIORAL REHABILITATION APPROACH

1. Introduction

1.1. Pain as an emotional experience

1.2. Acute pain versus chronic pain: a fruitful distinction?

1.3. Historical land marks

1.3.1. The Gate Control Theory of pain

1.3.2. The Operant Paradigm of chronic pain

1.4. The behavioral versus the traditional disease model

1.5. Chronic pain and rehabilitation medicine: A combined Behavioral Rehabilitation approach

1.6. Chronic low back pain

1.7. Conclusions

2. Chronic pain and the three systems-model of emotions. A critical examination

2.1. Introduction

2.2. The three-systems model of emotions

2.3. The three response systems of chronic pain

2.3.1. The overt-motoric response system

2.3.2. The physiological response system

2.3.3. The verbal-cognitive response system 37

2.4. Concordance or discordance?

2.5. Synchrony or desynchrony? 44

2.6. Consequences for chronic pain assessment 46

2.7. Consequences for chronic pain treatment 48

2.8. Discussion

PART II: BEHAVIORAL ASSESSMENT OF CHRONIC PAIN IN REHABILITATION

3. The dimensions and components of observed chronic pain behavior

3.1. Introduction

3.2. Subjects

3.3. Procedure 
3.4. Results

4. Assessing the components of chronic pain behavior: The development of a Checklist for Interpersonal Pain Behavior (CHIP)

4.1. Introduction

4.2. Study I: Factor structure 66

4.2.1. Subjects

4.2.2. Procedure

4.2.3. Results and discussion 67

$\begin{array}{ll}\text { 4.3. Study II: Intra-rater reliability } & 71\end{array}$

4.3.1. Subjects

71

4.3.2. Procedure

71

4.3.3. Results and discussion $\quad 72$

$\begin{array}{ll}\text { 4.4. Study III: Inter-rater reliability } & 72\end{array}$

$\begin{array}{ll}\text { 4.4.1. Subjects } & 72\end{array}$

$\begin{array}{ll}\text { 4.4.2. Procedure } & 72\end{array}$

4.4.3. Results and discussion $\quad 72$

4.5. Study IV: Validity of CHIP-DM and CHIP-NC $\quad 73$

$\begin{array}{ll}\text { 4.5.1. Subjects } & 73 \\ 4.5 .2 . & 73\end{array}$

$\begin{array}{ll}\text { 4.5.2. Procedure } & 73\end{array}$

4.5.3. Results and discussion $\quad 74$

4.6. Study V: Validity of CHIP-DM, CHIP-VC, and CHIP-NC 75

$\begin{array}{ll}\text { 4.6.1. Subjects } & 75 \\ \text { 4.6.2. } & 75\end{array}$

$\begin{array}{ll}\text { 4.6.2. Procedure } & 75\end{array}$

4.6.3. Results and discussion $\quad 76$

4.7. Study VI: Validity of CHIP-N and CHIP-D 77

$\begin{array}{ll}\text { 4.7.1. Subjects } & 77\end{array}$

$\begin{array}{ll}\text { 4.7.2. } & \text { Procedure } \\ & 77\end{array}$

$\begin{array}{ll}\text { 4.7.3. Results and discussion } & 77\end{array}$

$\begin{array}{ll}\text { 4.8. General discussion } & 78\end{array}$

5. Validity of a Dutch version of the Pain Behavior Scalle (PBS) 81

5.1. Introduction 81

5.2. Characteristics of the Dutch version (PBS) 82

5.2.1. Method 82

5.2.2. Results 85

5.3. Intra-rater reliability: Study I 85

5.3.1. Method 85 
5.4. Intra-rater reliability: Study II 85

5.4.1. Method 85

5.4.2. Results 86

5.5. Inter-rater reliability 86

5.5.1. Method " 86

5.5.2. Results 86

5.6. Validity 86

5.6.1. Method 87

$\begin{array}{lll}\text { 5.6.2. Results } & 87\end{array}$

5.7. Discussion 87

6. Behavioral assessment of residual health behavior in chronic low back pain patients through the pain-rest contingency $\begin{array}{ll}\text { principle } & 89\end{array}$

6.1. Introduction 89

6.2. Pilot study: the Motoric Skills Observation Scale (MOSOS) 92

6.3. Development of MOSOS-I 92

6.4. Development of MOSOS-II 93

6.5. Development of MOSOS-III 93

6.5.1. Subjects 94

6.5.2. Procedure 94

6.5.3. Results 95

6.6. Characteristics of MOSOS scores 97

$\begin{array}{ll}\text { 6.6.1. Subjects } & 97\end{array}$

$\begin{array}{ll}\text { 6.6.2. Procedure } & 97\end{array}$

6.6.3. Results 97

6.7. Reliability of MOSOS 100

$\begin{array}{ll}\text { 6.7.1. Subjects } & 100\end{array}$

6.7.2. Procedure 101

6.7.3. Results 101

6.8. Discriminative value of MOSOS 101

$\begin{array}{ll}\text { 6.8.1. Subjects } & 101\end{array}$

6.8.2. Procedure 101

6.8.3. Results 102

6.9. Construct validity of MOSOS 104

$\begin{array}{ll}\text { 6.9.1. Subjects } & 104\end{array}$

$\begin{array}{ll}\text { 6.9.2. Procedure } & 104\end{array}$

6.9.3. Results 104

6.10. Discussion 106 
7. Development of a pain cognition questionnaire

7.1. Introduction

7.2. Development of the Pain Cognition List (PCL)

7.2.1. Pilot study

7.2.2. Subjects

7.7. Procedure

7.7. Results

7.3. Examination of the relationship between factor scores and individual differences

7.4. Reliability

7.5. Validity

7.5.1. Criterion-related validity

7.5.2. Predictive validity:

7.6. Discussion

PART III: INTERDISCIPLINARY TREATMENT OF CLBP IN REHABILITATION

8. A behavioral treatment for sitting and standing intolerance in a patient with chronic low back pain

8.1. Introduction

125

8.2. Subject

8.3. Clinical procedure

8.4. Behavioral program

8.5. Statistical analysis

8.6. Results

8.7. Discussion

9. Effectiveness of inpatient operant and operant-cognitive treatment for chronic low back pain I:

Statistical significane

9.1. Introduction

9.2. Method

9.2.1. Subjects

9.2.2. Drop-outs

9.2.3. Biographical data

9.2.4. Personality characteristics

9.2.5. Outcome measures

9.2.6. Statistical procedures

9.2.6.1. Variable reduction technique

9.2.6.2. Testing the hypotheses 
9.3.1. Variable reduction

9.3.2. Immediate treatment effects: Comparison between the $O P$ and OC groups

9.3.3. Immediate treatment effects: Comparisons among the three groups under study

9.3.4. Long-term treatment effects: Six month follow-up

9.3.5. Long-term treatment effects: One year follow-up

9.4. Discussion

10. Effectiveness of inpatient operant and operant-cognitive treatment for chronic low back pain II: Clinical significance 161

10.1. Introduction

10.2. Examination of clinically significant pre-treatment-posttreatment change

10.2.1. Method

10.2.2. Results

10.3. Examination of clinically significant post-treatment level

10.3.1. Method

10.3.2. Results

10.4. Examination of clinically significant follow-up levels

10.4.1. Method

10.4.2. Results

10.5. Discussion

11. Effectiveness of inpatient operant and operant-cognitive treatment for chronic low back pain III:

Broad-spectrum analysis

11.1. Introduction

11.2. Method

11.2.1. Measures

11.2.2. Statistical analyses

11.3. Results

11.3.1. Broad-spectrum analysis: clinical picture of the sample

11.3.2. Immediate treatment effects; comparison between the $O P$ and $\mathrm{OC}$ groups

11.3.3. Immediate treatment effects: comparison among the three groups under study

11.3.4. Long-term treatment effects: 6 -months and 12-months follow-up 
11.3.5. Relationship among problem areas and classes of clinically significant change, and post-treatment level

11.3.6. Relationship among problem areas and change in health behavior

11.4. Discussion

12. Desynchrony following operant and operant-cognitive treatment for chonic low back pain

12.1. Introduction

12.2. Method

12.3. Results

12.4. Discussion

13. Methodological justification

13.1. Introduction

13.2. Internal validity

13.3. External validity

13.4. Statistical analyses

201

13.4.1. Variable reduction

201

13.4.2. Power

202

13.5. Conclusion

204

PART IV: GENERAL DISCUSSION AND CONCLUSIONS

APPENDIX A. AN OPERANT TREATMENT PROGRAM FOR CLBP PATIENTS

APPENDIX B. A COGNITIVE TREATMENT PROGRAM FOR CLBP PATIENTS

LITERATURE

LIST OF FREQUENTLY USED ABBREVIATIONS

SUMMARY

SAMENVATTING

DANKWOORD 
PART I

\section{CHRONIC PAIN AND}

THE BEHAVIORAL REHABILITATION APPROACH 



\section{CHAPTER 1}

\section{Introduction}

\subsection{Pain as an emotional experience}

The International Association for the Study of Pain (IASP, 1986) defined pain as "an unpleasant sensory and emotional experience associated with actual or potential tissue damage, or described in terms of such damage." An important conceptual feature of this definition is that pain consists of both sensory and emotional components. Moreover, the notes on term usage of the same IASP taxonomy (1986) clearly state that pain must be viewed as a psychological experience and not as the physiological activity occurring simultaneously: "Activity induced in the nociceptor and nociceptive pathways by a noxious stimulus is not pain, which is always a psychological state, even though we may well appreciate that pain most often has a proximate physical cause" (p. S217).

Quite rightly, Weisemberg (1987) stated that this worldwide accepted definition lays down a challenge to psychologists who supposedly are the experts at dealing with psychological phenomena. Almost a century ago, the relation between pain and psychology was already described by Strong (1895) who studied the psychic reaction of pain and suffering. Since this publication relative little progress has been made. A comprehensive psy. chological theory is still lacking (Weisemberg, 1987).

This study has no pretensions to provide such a theory. It is an attempt to summarize recent clinical research findings and bring them together into the heuristics of the three-systems model of emotions. Chronic pain is conceptualized as a pathological emotion that can be approached following three relatively independent response systems: overt-motoric behavior, verbal-cognitive responses, and psychophysiological reactions. This framework will subsequently be used in chronic pain assessment and treatment. Arguments in favor of the triple response mode framework are provided in chapter 2 . In this introductory chapter, common issues the clinical researcher interested in pain and chronic low back pain (CLBP) has to face are briefly highlighted. They include the distinction between acute 
and chronic pain, the emergence of the behavioral model, historical landmarks in the behavioral approach to chronic pain, the implementation of pain treatment in rehabilitation medicine, and the problem of CLBP.

\subsection{Acute pain versus chronic pain: a fruitful distinction?}

In contrast to acute pain, which almost always occurs after tissue damage, chronic pain is defined as pain that persists beyond the usual course of an acute disease or a reasonable time for an injury to heal or pain that recurs at intervals for months or years. Furthermore, acute and chronic pain are not only different in time scale but are fundamentally different in kind. The pathophysiological, psychological and affective responses to chronic pain are quite different from those to acute pain. For example, in most chronic pain patients the autonomic reflex responses decrease progressively and eventually disappear. In contrast, vegetative signs increase over time (Bonica, 1985). Although some clinicians use the arbitrary figure of 6 months to designate pain as chronic, some authors do not agree. They choose the assumed healing time as criterion for chronicity. Many acute diseases or injuries heal in 2 to 4 weeks. In such conditions, if pain is still present some weeks after cure should have been achieved, it can be considered chronic pain.

In the context of persisting pain complaints also the terms 'chronic benign pain", "idiopathic pain syndrome', "chronic intractable benign pain syndrome' and 'chronic pain syndrome' are used (Karoly \& Jensen, 1987). The first three terms emphazise the discrepancy between the organic findings and the pain complaints. We prefer not to use the term "benign' as chronic pain is never benign. It always has a disruptive effect on a person's life. "Idiopathic" means "peculiar to the individual, arising spontaneously or from an obscure or unknown cause" (Websters New Collegiate Dictionnary, 1981) and suggests the absence of an organic cause. 'Intractable' means "not easily relieved or cured" and points to failure to find a suitable medical treatment. All three terms refer to the mind-body dualism as if the presence of nociception would be a guarantee that no emotional factors might be responsible for the existing pain complaints. A distinction should be made between the factors that have initiated the pain problem and those that are maintaining it. In the course of time, a shift may occur from nociception to environmental factors as the controlling event. The term 'Chronic Pain Syndrome (CPS)' is the most neutral, reflects the multidimensional character of chronic pain problems, and will be the term of choice in the following chapters. Table I shows the general characteristics and the epiphenomena of CPS. 
A. General characteristics

The Chronic Pain Syndrome (CPS) is defined as an ongoing problem with pain that:

1. Cannot be shown to be causally related in the here and now with any active pathophysiologic or pathoanatomic process.

2. Has a history of generally ineffective medical and surgical intervention in the pain problem.

3. Has come to be accompanied by disturbed psychosocial functioning that includes the pain complaint and the epiphenomena that accompany it.

\section{B. Epiphenomena}

1. Substance use disorders of varying severity with their attendant central nervous system side effects.

2. Multiple operations or pharmacological treatments with their own morbid side effects separate from those related in 1.

3. Escalating physical incapacity secondary to pain, anticipating pain, and fear this pain is a signal of increasing bodily harm and damage.

4. Increasing hopelessness and helplessness as increasing dysphoria does not give way in the face of mounting numbers of 'newer' or different therapies.

5. Conflicts with medical care delivery personnel (doctors, nurses, therapists, and technicians) with resulting dissatisfaction and/or different therapies.

6. Interpersonal conflicts with significant others.

7. Unpleasant and lasting mood and affect changes.

8. Decrease in feelings of self-esteem, self-worth, and self-confidence.

9. Escalating social withdrawal and increased loss of gratification from these interactional inputs.

10. Decreased ability to obtain pleasure from the life process, contributing to profound demoralization and, at times, significant depression.

From: J.J. Pinsky and B.L. Crue, (1984).

Based on a series of epidemiological studies, Schmidt (1986) proposes to change the time criterion for CLBP from 6 months to 2 months. He states that after this period $95 \%$ of the acute low back pain patients are cured. Moreover, "such an advancement of the chronicity criterion could be advantageous for the study of the psychological and social mechanismas that lead to prolonged chronicity which in turn could result in a policy more directed towards prevention." (Schmidt, 1986, p.10).

The present author believes that too much attention is paid to a classification based on pain duration and the presumed role of pathological bodily states. At least two observations lead to the conclusion that such classification is not a fruitful one, and distract from more essential issues. First, the internationally accepted definition says that pain is a sensory and 
emotional experience, and not only chronic pain. At any time in the pain history, pathological organic states have to be carefully looked at, but they poorly contribute to the chronicity process. Second, the group of pain patients receiving the label 'chronic pain syndrome' is far from homogeneous. An illustration of the limited usefulness of the term is provided by Crook \& Tunks (1985). Using an epidemiological study, they compared two groups of randomly selected chronic pain patients, attending a university pain center and a private family practice, respectively. In agreement with earlier findings (Chapman et al., 1979), the investigators found considerable more emotional disturbance in patients attending a university pain center:

"They report a greater intensity and constancy of pain, with more difficulty in activities of daily living. They experience somatic symptoms such as loss of appetite, reduced energy, and diminished libido along with more typical depressive findings. They frequently choose social withdrawal as a response to pain. The long-term consequences of their problem include job loss, litigation, occupational disability, and alcohol or drug abuse. They are frequent users of health services including family physician as well as other health care personnel" (p. 162).

These results suggest that the 'chronic pain syndrome' has not the same meaning for pain sufferers attending pain centers as for individuals who suffer persistent pain but who are not referred to such centers. Moreover, the authors also found that the pain clinic patients scored higher on indices tapping self-statements of physical, emotional, and social dysfunctioning, but they also found a lack of correlations between the pain-related and the psycho-social and functional indices. The authors suggest that the individuals who suffer the coincidence of both psycho-social disturbance and painful symptoms, are more likely to become chronic pain clinic patients. These patients probably have greater adaptive problems. These findings receive support from a study reported by Deyo et al. (1988) who compared chronic pain patients recruited through publicity in the lay media with patients who already received care in a pain clinic. The findings suggested that the latter were less often employed, had less favorable personality profiles, and were more likely to have had surgery or prescriptions for analgesics. These differences existed despite similarly long durations of pain and equivalent sites of pain (all low back) for the two groups. The just mentioned studies suggest that it is insufficient to simply describe patients as having "chronic pain' since the label includes subjects with widely varying traits and prognoses. 
As prevention will increasingly become an important part of the work of health care providers, research aimed at identifying factors that lead to chronicity will need more attention than it has received so far. Murphy \& Cornish (1984), for example, examined whether low back pain patients who develop chronic pain are measurably different from those who recover from an acuie episode. They conclude that compared to an acute pain patient, a prechronic patient complains of pain at numerous body sites, more often has deep musculoskeletal rather than peripheral pain, is highly anxious in the acute stage, and does not have an activity level sufficient to preclude a preoccupation with pain. The generalizability of the study, however, is restricted by the relative small sample size $(\mathrm{N}=48)$ and the inclusion of only male veterans as subjects.

Some authors have introduced a pain classification based on patient coping style and significant other reaction patterns in addition to duration of complaint and putative causal agent (Crue, 1985; Keefe \& Brown, 1982; Pinsky \& Crue, 1984). This classification includes 6 categories: acute, recurrent acute (intermittent), ongoing acute (progressive), prechronic, chronic benign (persistent) and chronic intractable benign pain syndrome. Although such a classification presents a significant improvement, the IASP Subcommittee on Taxonomy (1986) did not follow this classification.

Interestingly, psychology has dealt with chronic pain states but rarely with acute pain. This might be explained by the fact that in acute pain states, enough physical pathology exists for physicians to deal with. In that case, however, environmental, behavioral or emotional factors that may lead to chronicity are overlooked. Fordyce et al. (1986) suggested an acute pain approach based on a behavioral model. In their prospective, controlled study they examined the effect of behavioral methods developed for evaluating and treating chronic pain on acute back pain. Patients coming for treatment in a medical clinic were randomly assigned to a traditional treatment regimen or to a treatment procedure based on behavioral approaches to clinical pain. In the traditional regimen, a given level of activity limit was prescribed. The end of this activity constraint was to be defined by the patient, according to how she/ he felt: i.e. "let pain be your guide". In the behavioral approach, a fixed number days of rest was prescribed, and exercices were set to begin on a pre-determined day, with the number of repetitions and rate of increments specified, e.g.: "start on day five with 6 repetitions of each exercise and increment 2 repetitions each session.". The data of the 9-12 month follow-up period suggest that the traditional regimen resulted in people describing themselves as less well off, whereas the behavioral approach subject group had returned to their pre-injury level of functioning. 


\subsection{Historical landmarks}

It was only during the past 25 years that psychologists have been increasingly involved in the area of pain control and research. At least two important landmarks can be distinguished. 1. The Gate Control Theory (1965) and 2. Fordyce's operant paradigm (1976).

\subsubsection{The Gate Control Theory of pain}

In 1965, Melzack \& Wall formulated the Gate Control Theory of pain in its first version. Basically, the theory proposes that a neural mechanism in the dorsal horns of the spinal cord acts like a gate which can increase and decrease the nervous conduction from peripheral fibres to the central nervous system. When the amount of information that passes through the gate exceeds a critical level, it activates the neural areas responsible for pain experience and response. The Gate Control Theory has some interesting implications. First, it emphasizes parallel processing systems of sensory, affective and cognitive information. Second, it assumes descending pathways from higher order nervous systems (reticular and limbic system) towards the gate. Although its validity has not totally been proven yet, the gate-control model provides physiologic justification for considering psychological processes such as conditioning, attentional focus, expectancy, and emotionality (fear and depression) as important determinants of the pain problem.

\subsubsection{The operant paradigm of chronic pain}

About a decade later, Fordyce (1976) introduced his influential book 'Behavioral methods for chronic pain and illness'. His work stemmed from the obvious shortcomings of traditional health care approaches for resolving adequately many problems of chronic pain. Fordyce was the first to apply the operant conditioning principle originated by Skinner (1953) to the problem of chronic pain and to introduce the concept of 'pain behavior', referring to observable communication of pain and suffering. The basic assumption of his approach to chronic pain is that pain behaviors (as any kind of behavior) are operantly controlled by their consequences.

\subsection{The behavioral versus the traditional disease model}

If pain is viewed as an emotional experience, what can psychologists contribute to the conceptualization, diagnosis, and treatment of pain? Most people, including the majority of health care professionals, however, will 
search for a physical reason if a patient complains of pain. This perspective reflects the traditional disease model, and views pain behaviors as symptoms of an assumed underlying cause. The consequence of such a notion is that the alleged 'underlying cause' must first be corrected before the symptom, in this case pain behavior can diminish. This model is derived from the writings of René Descartes (1596-1650), philosopher of the French Enlightment. Descartes divided human existence into 'mind' and 'body'. For him, pain was a reflex response to a physical stimulus, predictable if the characteristics of the stimulus were known. In the clinical situation, the disease model claims the attention of the physician be it an anesthaesiologist, orthopedic surgeon, neurosurgeon or reumatologist on one side, and the psychiatrist on the other. This 'dualistic' model does reasonably well in cases of acute or chronic malignant pain. It begins to fail when chronicity is reached. By then, the spectrum of phenomena influencing the occurrence of pain will inevitably have broadened.

An alternative view is a behavioral or learning-based model. In contrast to the disease model, which evolved from clinical work and philosophical considerations of the nature of man, the behavioral model grew out of laboratory studies of behavior development and change. The behavioral model focuses on 'behaviors', and is based on learning paradigms. It emphasizes controlled manipulations of observable events, careful measurements, and the resolution of complex events into simpler components. Initially, the behavioral model focused on simple learning in animals, but gradually it expanded toward experimental approaches to the analysis of many complex human behaviors, including complex social and verbal interactions, and cognitive processes. Kanfer \& Philips (1970) distinguished 6 common assumptions of the behavioral model which we have summarized as follows:

1. The main focus is on behavior.

2. All behaviors are considered as being subject to the same learning principles.

3. The behavioral model attacks behaviors directly, and not the underlying disease processes that are said to cause symptomatic behaviors.

4. The behavioral model shares its methods of inquiry into human behavior with all other sciences.

5. The behavioral model requires the ability to make appropriate measurements.

6. Although programs for behavior modification always deal with current behaviors, the behavioral model recognizes the importance of past events in the formation of learned behaviors. 
Ad. 1.

According to Reynolds (1968), "Behavior is everything that organisms do." (p.5). From a pure 'behavioristic" point of view behavior included only observable motoric responses. The term 'pain behavior' therefore often refers to the overt-motoric responses of patients suffering pain. Private events such as 'thoughts', 'ideas', and 'images" that are not observable to anyone other than the person for whom they are occurring have been considered as 'covert' behaviors (Meichembaum, 1977, Cautela \& Kearny, 1986). Psychophysiological responses such as heart rate, muscle tension, galvanic skin response etc. have also been considered as behaviors, although their assessment is only possible by means of electronic devices. In their statement on behavioral assessment, Nelson \& Hayes (1979) comment: "behavioral assessment also includes (in addition to overt motor behavior) the measurement of physiological-emotional behavior and cognitive-verbal behavior.". Mischel (1968) writes: "we must consider what people do cognitively and affectively, not just motorically.". The three-systems model, originated by Lang (1968), refers to these behavioral components (overt-motoric, physiological, and verbal-cognitive) as partially independent, with little covariation. Chapter 2. provides an elaboration of this model in relation to chronic pain.

Ad. 2.

Three types of learning are considered important in developing and altering behavior: Operant conditioning, classical or respondent conditioning, and observational or social learning (Kazdin, 1980).

The operant learning principle refers to Thorndike's 'Law of effect' (Thorndike, 1932). Behavior which is followed by a positive reinforcement has a greater probability to increase in frequency, while behaviors that are not followed by this reinforcement will probably decrease in frequency (extinction). Fordyce (1976) was the first to apply the operant learning principle to chronic pain behavior. Many investigators have demonstrated that pain behaviors can be increased through operant conditioning (Cairns \& Pasino, 1977; Block et al., 1980; Fordyce et al., 1973; White \& Sanders, 1985).

The classical conditioning paradigm, originated by Pavlov (1927), is concerned with stimuli which automatically evoke responses. Through classical conditioning, a stimulus which is neutral, that is, does not automatically elicit a particular reflex, is made to elicit a reflex response. This process occurs by repeatedly pairing the neutral stimulus with a stimulus that does automatically elicit the reflex response. In classical conditioning, events or stimuli which precede behavior may control that behavior. Despite 
the importance of the classical conditioning paradigm, there is relatively little research carried out that examined the relation between classical conditioning and (chronic) pain behavior.

Observational or vicarious learning through modeling occurs when an individual observes a model's behavior but performs no overt responses nor receives direct consequences him/herself. By observing a model, a response may be learned without actually being performed (Kazd in, 1980). The performance, and maintenance of this learned behavior, however, is dependent on the consequences to the model and the observer. Observational learning is considered to play an important role in the acquisition of pain behaviors during child hood (Craig, 1978). A study of Turkat et al. (1983), for example, showed that subjects exposed to a high-avoidant model tolerated a pain stimulus for significantly fewer seconds and did significantly less work, compared to subjects who observed low-avoidant models.

Bandura (1977) has developed a social learning theory that is aimed at integrating aspects of different learning paradigms taking into account cognitive processes. In this theory, cognitive processes largely determine what environmental influences are attended to, how they are perceived, and whether they might affect future action. Causal attributions and selfefficacy expectations are the most common cognitive mediational processes. Although a sound 'cognitive' learning paradigm is still lacking, social learning theory has been applied to chronic pain (Turk et al., 1983).

\section{Ad. 3.}

The behavioral treatment of pain deals directly with pain behaviors conceptualized in the heuristic of the triple response mode. Operant treatments (e.g. Fordyce, 1976) focus on the modification of motoric pain behaviors, cognitive treatment programs (e.g. Turk et al., 1983) are aimed at altering the way pain patients think of their pain, and respondent techniques (Grzesiak, 1977, Linton \& Melin, 1983) are developed to reduce the elevated physiological arousal level which occurs in particular situations.

\section{Ad. 4.}

The traditional intra-psychic approach (such as Psycho-analytic theory and Trait theory) has proposed that the rules of theory construction and the methods common to natural sciences are inappropriate for collection of psychological data and verification of psychological principles. The behavioral model disagrees with this point of view, and emphasizes the importance of empirical examination of human behavior. This means that knowledge is acquired through systematic observation, formulation of hy- 
potheses, deduction and formulation of predictions, testing, and evaluation (Kerlinger, 1986).

One of the consequences of the empirical approach of the behavioral model is that communication with other human sciences has been facilitated. During the last 10 years, the application of behavioral procedures to the treatment of physical illness has proliferated. Many medical centers and professional schools of dentistry now call in the help of behavioral psychologists to develop treatment programs. Additional reasons supposedly are: 1 . the growing appreciation for the psychological variables affecting 'physical health', 2. the realization that health is influenced by life styles and habits, 3 . the recognition that behavioral techniques can produce dramatic changes in a persons functioning, and last but not least, 4 . the observation that behavioral procedures are far less expensive than traditional alternatives, with far less side effects (Redd \& Rusch, 1985).

\section{Ad. 5.}

In contrast with traditional assessment, which typically emphasizes the intra-organismic causes of behavior, behavioral assessors hold either a situational (e.g. Mischel, 1968) or an interactionist point of view that behavior is a result of environmental factors or of environmental factors interacting with organismic variables (Nelson \& Hayes, 1986). The focus of assessment is on what a person does, rather than on what he or she has (Mischel, 1968). Not only overt motoric behaviors, but also thoughts and feelings are products of environmental variables. In behavioral assessment, motor behavior, thoughts, and feelings are assessed as separate but equally important behaviors within the heuristic of the triple response mode. Behavioral assessment has the following three goals: 1 . the identification of the problematic responses, 2. the selection of treatment, and 3. the evaluation of treatment. The functional analysis is the classic strategy that links behavioral assessment and treatment: "It specifies the causes of behavior in terms of explicit environmental events that can be objectively identified and that are potentially manipulable (Ferster, 1965, p. 11).
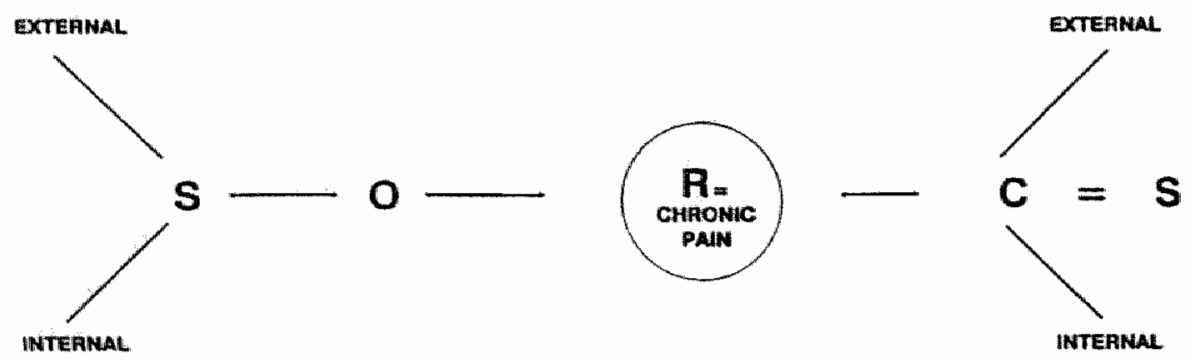

Figure 1.1: The SORC equation. 
The functional analysis is based on the behavioral equation SOR(K)C (figure 1.1) or Stimulus-Organism-Response-Contingency-Consequences (Kanfer \& Philips, 1970). $\mathrm{R}$ is the problematic behavior that is identified for modification. The other components represent the controlling variables of which this response is a function. The immediate environmental variables are represented as $S$ (theinternal or external stimuli that precede $R$ ) and $C$ (the consequences that follow R). A closer look to both stimulus conditions and consequences brings us to the distinction between internal and external events. Internal events are those that occur inside the organism and that can be detected through the process of proprioception. External events occur outside the organism and can be detected through perception. For example, in a patient suffering from rheumatoid arthritis different stimuli occur during flare-ups as compared to periods of relative stability. Consequently, his response may be different in those two circumstances. The same distinction can be made for the consequences. Taking pain medication as a motoric response may change the internal milieu, thereby affecting internal stimuli. At the same time, increased attention from the spouse may occur, which is an external consequence. The equation represents not a static but a dynamic, spiral system, in which consequences provide new stimuli for subsequent $S O R(K) C$ chains. The organismic variables are represented by $O$ and include both physiological status and past learning history. $K$ refers to the particular arrangement between the problematic behavior ( $R$ ) and its consequences ( $C$ ), also called contingency. $K$ is often omitted in the behavioral equation because it is situated on a higher level of abstraction than $S, O, R$, and $C$. The functional analysis in terms of the SORC-equation has been applied to the area of chronic pain (Fordyce, 1976; Groenman et al, 1986; Vlaeyen, 1985; Vlaeyen et al., 1987).

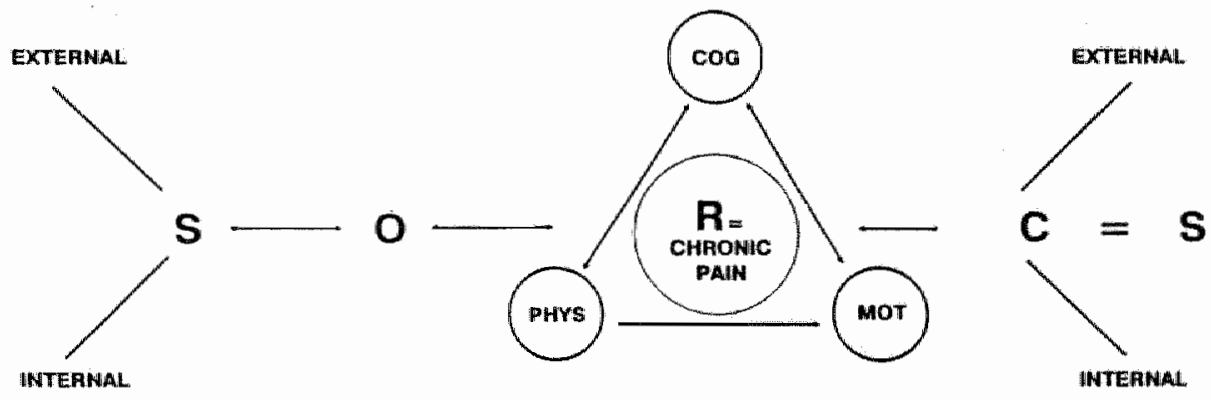

Figure 1.2: The extended SORC equation. 
Table 1. II: Response excesses and deficits.

\begin{tabular}{llll}
\hline & Motoric & Cognitive & Physiological \\
\hline Excesses & Pain behaviors & Catrastrophizing & $\begin{array}{l}\text { Stress-related } \\
\text { muscle tension }\end{array}$ \\
Deficits & $\begin{array}{l}\text { Lack of motoric health } \\
\text { behaviors }\end{array}$ & $\begin{array}{l}\text { Lack of Self-efficacy } \\
\text { expectations }\end{array}$ & $\begin{array}{l}\text { Lack of } \\
\text { muscle tone } \\
\text { (Disuse syndrom) }\end{array}$ \\
\hline
\end{tabular}

As mentioned in Ad.1., and further elaborated in chapter 2, the response R has at least three components or response systems (motoric, cognitive, and physiological). Figure 1.2 shows the extension of the SORC equation with the response systems. Each of these response systems can be subdivided in response excesses and response deficits (Table 1.II). Response excesses refer to behaviors that occur too frequently or too strongly. Response deficits refer to the responses which do not belong to the person's response repertoire anymore, or less frequently than needed, and most of which appear in healthy people.

To identify a response as excessive or deficient the context (S) in which it occurs must be considered. A person in a wheelchair might have to emit behaviors that healthy people would not do, but that would still be beneficial to that person.

Ad. 6.

The functional analysis focuses on a particular problem behavior or respons (such as pain), but is always to be situated in the context of a patients past learning history. In the functional analysis, the clinician tries to assess all the response excesses and deficits in the context of their antecedents and consequences.In addition, the broad spectrum analysis* presents the totality of problem behaviors and the assumed causal relationships among them. A broad spectrum analysis of pain consists of different levels of assessment and places the chronic pain problem in the broader context of other pathological emotions or problem areas, the environment, personality characteristics, and the patient's past history.

"Dutch behavior therapists use the term "holistische theorie", literally translated: 'holistic theory'. Because the English term 'holistic' has a wider connotation than the one used by Dutch behavior therapists, preference is given to "broad-spectrum analysis". 


\subsection{Chronic pain and rehabilitation medicine: $A$ combined Behavioral- Rehabilitation approach.}

Although most of the work of chronic pain assessment occurs at specialized university pain clinics, the behavioral management of chronic pain takes place in so-calied pain management centers. In most European countries, such as the Netherlands, however, experimental pain management programs have recently emerged in existing rehabilitation centers that are sometimes affiliated with a university hospital. As these programs appear to be very promising, National Health Councils are advising ministers of health to grant official accreditation to these programs. As Rehabilitation Medicine increasingly deals with behavioral pain management programs, a closer look at the integration between the rehabilitation and behavioral models, in relation to the problem of chronic pain, is desirable.

As mentioned before, the traditional "disease" model is considered to be limited to identification and the treatment of diseases. The restricted utility of this model appears to be due to the higher priorities given to 'curing' patients compared to 'caring' for patients. Caring requires a different problem-solving approach to clinical practice, its goals should be seen as restoring and/or maintaining independence. As Granger (1984) states:

"The unintended consequence of persisting with the disease model is likely to be the deterioration of social role competencies for persons with disabilities. That is, in the medical model the person with disabilities is often encouraged to maintain the sick-role" (p. 15).

The combined behavioral-rehabilitation approach gives the opportunity of blending medical and behavioral aspects of care. This approach means identifying how people improve, or learn skills through graded training, which is adapted to the learners' physical status. The skills are then used to cope better with demands of the environment and to reach the desirable level of independence.

The World Health Organization developed the International Classification of Impairments, Disabilities and Handicap (ICIDH) as a logical complement to the International Classification of Diseases. Rehabilitation Medicine is defined as "The combined and coordinated use of medical, social, educational and vocational measures for training or retraining the individual to the highest possible level of functional ability" (WHO, 1980). In the ICIDH, three concepts are distinguished: Impairment, Disability, and Handicap which are respectively defined as follows: 
Impairment: "any loss or abnormality of psychological, physiological or anatomical structure or function".

Disability: "any restriction or lack of ability (resulting from an impairment) to perform an activity in a manner or within the range which is considered normal."

Handicap: "a disadvantage for a given individual, resulting from an impairment or a disability, that limits or prevents the fulfillment of a role that is normal (depending on age, sex, social, and cultural factors) for that individual."

In a patient with low back pain, the impairment may be the 'herniated disc', the disability may include the inability to walk properly, and the handicap represents the social consequence of the disabilities, e.g. the dependence on social security benefits.

The definitions of the ICIDH concepts are not always followed consistently, and the concepts are used in different ways in different publications. Some authors use 'disability' instead of 'handicap', and others prefer 'functional limitation' rather than 'disability'. Even the term 'social security disability' has been used as the inability to engage in any substantial gainful activity (Wilson, 1990).

Often a causal relation among the concepts of Impairment, Disability, and Handicap is suggested. However, as Granger (1984) points out, impairment by itself should not be considered a necessary and sufficient cause for disability. In this chapter, the position is taken that the behavioral model provides alternative explanations as to why disability occurs, even when at the impairment level, little can be found. Similarly, the fairly measurable degree of disability may not be equated with the more relative degree of handicap. Not only is there variation in severity of impairment among individuals with the same diagnostic condition, but even among those with the same level of severity, individuals differ as to how well social role expectations are met. Rather than representing impairment-disability-handicap elements in a causal chain, they can be seen as "different planes of experience" (Wood \& Badley, 1981).

Figure 1.3 shows the relationships of factors including condition, assessment and interventions to the Impairment, Disability, and Handicap model. Figure 1.4 shows the integration of the rehabilitation model (IDH) and the behavioral model (SORC). Both figures will be discussed simultaneously. There are basically three levels of concern: the organ level, the person level, and the societal level. Clinicians working at the organ level 
LEVEL:

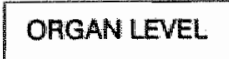

CONDITIONS:

IDH-MODEL:

INTERVENTIONS:

Medical and Restorative Therapy
PERSON LEVEL

SOCIETAL LEVEL

ROLE ASSIGNMENT

Enwironmental

Socletal

Cognittive

Psycho-

physilotogical

Deficits and

Excesses

contribute to
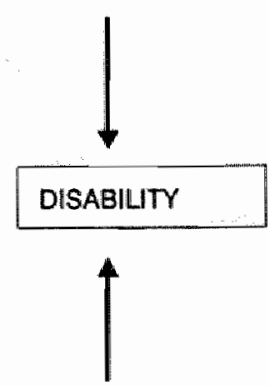

Behavloral

Management

Rules and Norms create
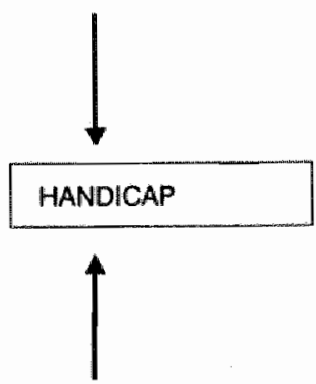

Supportive

Services and

Soclal Policy

Changes

Figure 1.3: Relationships of factors including condition, assessment, and interventions to the Impairment, Disability, and Handicap model (Based on Granger, 1984).

are chiefly concerned with pathology and its manifestations in impairments. Within the behavioral model, the organ level is situated in the Organism (O).

The concept of Disability embodies the idea of restrictions in tasks and activities at the person level. The corresponding concept in the behavioral model is the Response (R). It is the result of impairment, other characteristics of the organism (O), and the environment (C) in a specific situation (S). For example, a person with back pain who relies on a wheelchair for mobility, and remains in bed most of the time, may be disabled because this behavior (R) provides him a way of receiving extra attention from the environment $\left(C_{1}\right)$, or it avoids immediate pain increase $\left(C_{2}\right)$. 


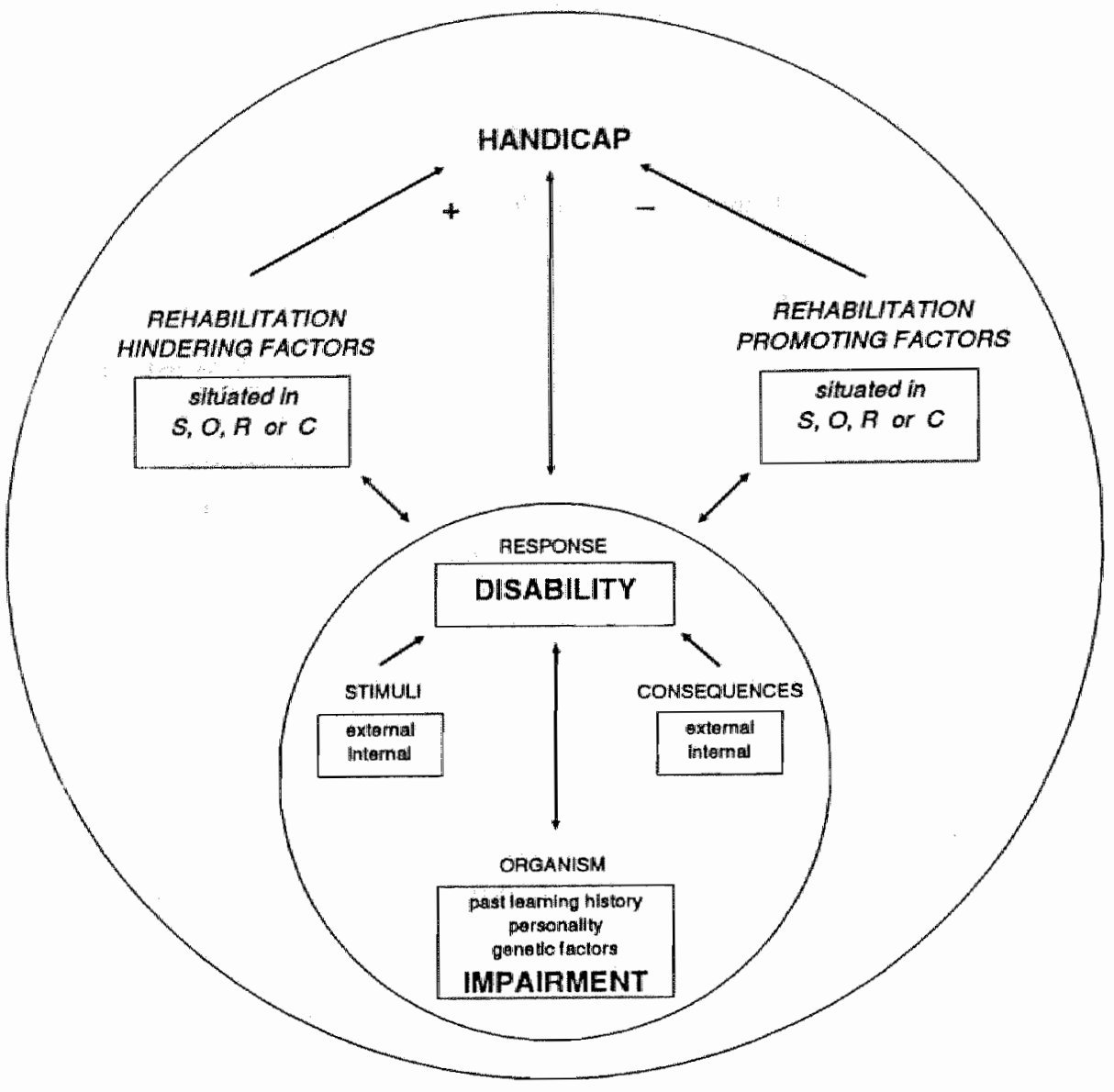

Figure 1.4: An integration of the rehabilitation model (IDH) and the behavioral model (SORC). The inver circel refers to the "functional analysis", the outer circel to the 'broad-spectrum analysis'.

Handicap accrues by virtue of being unable to perform according to social roles that are expected within the individual's context. From the behavioral perspective, handicap is one of the (sometimes long-term) negative social consequences $(C)$ of the disability. Handicap can also exist without disability. When a salesman is denied the opportunity for work due to facial disfigurement from severe burns, handicap results directly from im- 
pairment, without disability. On the other hand, disability is not always a sufficient condition for a handicap.

Two new hypothetical concepts will be introduced that, in interaction with disabilities, may determine the occurence of a handicap. These are called Rehabilitation-Promoting* (RP) factors, and Rehabilitation-Hindering $(\mathrm{RH})$ factors. As the terms suggest, they can respectively promote or hinder the rehabilitation process. RP factors are those factors which predict succesfull rehabilitation outcome, while RH factors are predictive for unsuccesful rehabilitation. From the behavioral perspective, both factors can be situated in the Stimulus, the Organism, the Response and the Consequences, and are assessed in the broad-spectrum analysis. A chronic low back pain patient who also has a social phobia, is likely to relapse after treatment focused on the pain complaints only. The phobia (which can be presented in a SORC equation) is a hindering factor that need to be assessed and treated as well. If not, the phobic complaint can increase because of increased confrontation with the phobic stimuli, in this case the people the patient meets when undertaking more activities outside the home. It is also possible that the pain complaints, rather than the phobia, are likely to increase as a way to avoid the phobic stimuli. Most chronic pain patients will resist treatment for complaints other than the pain. However, in our experience many patients agree to such treatment when it is presented as a logical continuation of care immediately following a rehabilitation pain management program. More stable personality characteristics (situated in O) can hinder the rehabilitation process as well. The Minnesota Multiphasic Personality Inventory (MMPI) has been used by several authors as a predictor for treatment outcome (Moore et al. 1986, Love \& Peck, 1987). The results, however, are unconclusive.

So far, little attention has been given to Rehabilitation-Promoting factors. One can think of cooperative family members, creativity, sense of humor (DeAngelis, 1989; Lefcourt \& Martin, 1986), self-efficacy (Bandura, 1981), or sense of coherence. Creativity has sometimes been cited as an area of human endeavor that is beyond the scope of behavior modification. However, a number of studies have examined creativity as behavior that can be developed with behavior modification techniques (Winston \& Baker, 1985). Self-efficacy is the belief that one will accomplish one's goals. Sense of coherence is defined as a personal orientation related to effective coping and good health. Persons with a high level of sense of coherence see their

* The term Rehabilitation is used here in the broadest possible way. Rehabilitationpromoting factors also operate outside the Rehabilitation Center. The terms Healthpromoting and Health-hindering factors might be used interchangeably then. 
life as meaningful, see themselves as having the resources to cope with demands placed on them, and see the world as being understandable and making sense to them. Both self-efficacy and sense of coherence are shown to be good predictors of response to a pain management program (Dolce et al., 1986; Petrie \& Azariah, 1990).

Hindering and promoting factors can also be situated in the social environment $(S$ and $C)$. The willingness of spouses to cooperate with the rehabilitation program, is an essential factor as to the success of treatment. Therefore many multidisciplinary pain management programs strictly require the participation of the spouse.

Finally, interrelationships exist among impairment, disability, and handicap to the extent that not only do disability and handicap result from impairment but the latter may at times result from disability and handicap. For example, a wheelchair reliant individual who does not perform the usual range of activities because of avoidance of pain or responsibilities, may be more impaired than necessary by physiological deconditioning as well as by psychological deficits.

\subsection{Chronic low back pain (CLBP)}

This study deals with CLBP which is low back pain (LBP) presented by people who suffer from the chronic pain syndrome (see paragraph 1.2.). The diagnosis and the treatment of low back pain is among the most difficult, demanding and discouraging problem in medicine today. LBP is pain which is felt in the lumbosacral region of the spinal column. Nerves, periosteum, periosteal tendon insertions, spinal ligaments, articulations, and muscles of the low back are all endowed with sensory fibres and can cause pain in response to mechanical or inflammatory irritation. LBP can occur with or without a clear somatic etiology. Congenital disorders, traumatic injuries, inflammatory diseases, circulatory disorders, degenerative diseases as well as lesions in other organs such as a kidney infection, bowel obstruction and uterine fibroids can cause LBP. These conditions are relatively rare and their exclusion can usually be made by careful clinical examination. The term 'sciatica' is reserved for a radiating lower extremity pain which extends below the knee. Sciatica implies compression or injury to a spinal nerve or the sciatic nerve itself (Belkin, 1985). Although the majority of low back pain arises from a mechanical dysfunction of the lumbosacral spine, there are numerous etiologies which can be allocated to one of the following categories: viscerogenic, vasculogenic, neurogenic and spondylogenic (McNab, 1977). The category 'psychogenic' is also used, but 
unfortunately, only in the absence of any significant evidence of organic pathology. The term 'psychogenic' also refers to the dualistic 'disease' model, and is not being used by behaviorally oriented clinicians and scientists.

In the current health care system, the treatment of acute low back pain may be the logical consequence of the diagnosis. In many cases, or when the exact etiology is unknown, conservative and non-invasive medical treatment, such as bedrest and analgesics, is initiated. When these fail, more invasive kinds of medical treatment may follow, some of the most commonly used are: mobilization and manipulation, percutaneous and epidural injections with local anesthetics and steroids, chemonucleolysis, and surgery including laminectomy, disc excision, decompression, and fusion of the vertebra.

Our study only deals with CLBP in which the aforementioned conditions are absent or in which they do not totally account adequately for the pain problem. Without denying the value of the medical treatments in many cases, this study will focus on another approach, Behavioral Rehabilitation, which is not only based on the diverse medical etiologies, but departs from the learning theory as it can be applied to CLBP problems. Behavioral Rehabilitation is considerate of medical etiologies which are considered part of the 'organism' $(\mathrm{O})$ possibly leading to specific internal stimuli (S).

As mentioned before, CLBP is not a disease, and impairments are not always found (Nachemson, 1979; Flor \& Turk, 1984). Studies by Lindequist et al. (1984) and Mellin et al. (1984) have shown that purely somatically oriented treatments for CLBP may be discouraging. Instead of a somatic etiologic model, the behavioral rehabilitation model often can be applied. For example, a CLBP patient can be disabled because of his long lasting overt motoric pain behaviors. In the acute phase, distorted gait may result from a structural or a mechanical defect, or as a way to reduce the pain which is caused by nociception. After healing time has passed, however, the distorted gait may persist by virtue of the immediate consequences to which they lead: diminished pain, increased attention from others, time out from unpleasant situations, etc. After a certain time, disuse of the musculature may lead to a substantial skill deficit for normal gait. The patient may no longer know how to walk properly. In this case a disability occurs, without serious impairments. In some cases, this disability may cause a serious handicap. The focus of treatment is on the reduction of disability and the handicap. This is also the area in which rehabilitation medicine operates.

Based on his epidemiological study on the natural history of LBP, the Scottish orthopaedist Gordon Waddell, winner of the 1987 Volvo Award in 
Clinical Sciences, has formulated interesting findings some of which are briefly summarized:

1. Low back pain by its natural history appear to be a universal, benign, self-limiting condition. At some stage in their life, $80 \%$ of the human race will experience LBP. As many as $60 \%$ of the normal population have experienced some degree of LBP in the past year, although they will not necessarily have seen a physician or lost time from work. A total of 80 to $90 \%$ of attacks of LBP recover in about 6 weeks, irrespective of the type of treatment. These observations correspond with results of other epidemiological studies (Haanen, 1984; Flor and Turk, 1984; Nachemson, 1979; Vällfors, 1985) which also indicate that the chance of recidivism after the first LBP attack is about $80-90 \%$.

2. Low back disability, as opposed to LBP, appears to be a recent Western epidemic that is not explained by any demonstrable change in physical disorder. Low back pain causes the greatest problem in the middle working years of life, does not progressively increase with age, and does not correspond to age-related changes of disc-degeneration.

3. Conventional medical treatment for LBP has failed, and the role of medicine in the present epidemic must be critically examined.

\subsection{Conclusions}

Chronic pain is considered an emotional experience, and a psychological state for which a sound psychological model is still lacking. A considerable bulk of literature, however, suggest that the behavioral model offers the foundations for a better insight in how pain can become a persisting problem. Moreover, two historical land marks underscore the importance of the behavioral model as an integrating model linking both the concepts of "mind" and "body". From this perspective, the duration of the pain complaint, and the limited contribution of organic anomalies to the explanation of the pain problem, appear to be weak and unsatisfactory criteria to differentiate between acute and chronic pain. The question has even been raised whether such a distinction is fruitful. Attempts to look at the way patients and their environment are dealing with the pain, irrespective of pain duration and presence of objective physical findings, appear to be far more promising. 
Unfortunately, some clinicians still attune their treatment to the distinction between chronic pain due to underlying tissue pathology and chronic pain without such pathology. Very recently, Yanagida (1990), for example, proposed to use the term "chronic pain" only for the second type of pain, for which behaviorally oriented therapies should be the treatment of choice. Yanagida's view has been criticized by several authors including Cicala (1990), Gallagher (1990), Vlaeyen (1990) and Fishbein \& Rosomoff (1990).

Although definition issues are important, the question "do pain patients receive the treatment that is most beneficial for them?" is more relevant. The range of treatments for pain has been expanded significantly and yet the disability epidemic has not diminished (Aronoff, 1989). Our patient care can be improved with a better match between the pain profile and the treatment provided. Such a pain profile, designed from a Behavioral Rehabilitation perspective, provides a more comprehensive picture of the pain than a diagnosis based on the presence of tissue pathology only.

The case of CLBP is prototypic for people with CPS. CLBP has become an epidemic in western society that causes the greatest problems in the working middle years of life. Because of its magnitude of subjective suffering, as well as its gigantic expenditures, modern medicine has to find a way to cope with this disability. Once pain has become a chronic problem, Behavioral Rehabilitation, the action of which is focused on diminishing pain behavior and related disabilities, and restoring well behavior and functional abilities through behavioral management programs, offers the best conditions that are needed for the pain problem to be brought back to reasonable proportions. One study estimated that for every dollar spent in rehabilitation 10 dollars are saved on other costs (i.e. future medical costs and disability; Brown \& Sons, 1987). It is expected that what has been learned from inpatient treatment evaluation research can be applied to outpatient settings. Similarly, and already illustrated by Fordyce et al. (1986), behavioral principles can be succesfully applied in the treatment of acute pain states. In the future, when succesful actions for prevention have been developed as well, the role of the first line care will increasingly come into the picture. This situation has not been reached yet, at least not in the Netherlands. 



\section{Chronic pain and the three-systems model of emotions: a critical examination*}

\subsection{Introduction}

The purpose of the present chapter is to examine the applicability of the three-systems model, thus far applied exclusively to fear and anxiety, to the chronic pain syndrome. In this chapter, a rough map of the terrain will be sketched in order to locate the paths that connect chronic pain to emotion.

The notion that pain, be it acute or chronic, can be considered an emotion, or that it is at least influenced by emotional factors, is not new at all. Marschall (1894), inspired by Aristoteles' writings, introduced the so-called 'affect theory of pain', in which pain was the accompanying affective dimension of different kinds of sensory input. Von Frey (1896), by contrast, considered affect only as a secondary reaction to pain. Sherrington (1906) took an intermediate position and viewed pain as an experience with both sensory and affective factors being present simultaneously. After the beginning of the twentieth century, scientific attention to the affective dimension of pain stagnated in favor of rapid neurophysiological development. It was only in the 1950 's that the relationship between chronic pain and emotional experiences regained attention. Psychological explanations were formulated by Kolb (1954) for phantom pain, by Szasz (1957), who stressed the role of perceived impairment of the physical integrity for all types of pain, and by Engel (1959), who associated pain with feelings of aggression, guilt, loss and depression. These formulations were hypothetical and only supported

\footnotetext{
"This chapter is a modified version of Vlaeyen, J.W.S., Snijders, A.M.J., Schuerman, J.A., van Eek, H., Groenman, N.H., \& Bremer, J.J.C.B. (1989). Chronic pain and the three-systems model of emotions. A critical examination. Critical Revieus in Physical and Rehabilitation Medicine, 2, 67-76.
} 
by casuistic studies. The most important explanatory model after 1959 is Melzack and Wall's gate control theory (1965). The assumption of these researchers is that pain can be characterized by three dimensions: a sensory-discriminative dimension, a motivational-affective dimension and a cognitive-evaluative dimension. They also assumed that higher cortical connections exist (for example, between the thalamus and the limbic system) which are involved in both discriminative and motivational aspects of the pain experience. The gate control theory has hereby figuratively opened the gate for research on the role of psychological variables modulating and influencing pain. The gate-control theory, however, provides an inadequate account of the overt-motoric dimension of chronic pain. According to Melzack \& Wall, overt behavior is closely associated with the physiological component of pain. The absence of close association between its different component parts appears to be a typical feature in chronic pain states. This will be outlined in the section on discordance and desynchrony.

Today, pain is defined not only as a sensory experience but also as an emotional one. The International Association for the Study of Pain (IASP, 1986) proposed the following definition of pain: "an unpleasant sensory and emotional experience associated with actual or potential tissue damage, or described in terms of such damage." Swanson (1984) took this idea a step further and introduced the conceptualization of chronic pain as a third pathologic emotion, in addition to anxiety and depression. Based on the systems theory and the use of the analogy model, Swanson compared acute pain to fear and grief, and chronic pain to anxiety and depression. The analogies refer to the transitional changes that occur when acute becomes chronic, that is when fear (acute) becomes anxiety (chronic) and when grief (acute) becomes depression (chronic): the absence of an external cause, physiological changes and the increasing complexity. Moreover Swanson referred to similarities between psychological treatments applied to anxiety, depression and chronic pain. Based on these analogies, the existence of three "pathological" emotions is suggested: anxiety, depression and pain, which can occur in both an acute and a chronic form.

In the literature on chronic pain conceptualization, pain is often approached as a trimodal condition (Bakal et al., 1984; Cautela, 1977; Keefe, 1982; Mayer et al., 1986; Melamed, 1984; Philips \& Jahanshahi, 1986; Sanders, 1979; Vlaeyen et al., 1987). Sanders (1979), for example, proposed to conceptualize pain as "an interacting cluster of gross motor, cognitive and physiological responses".

The same trichotomy is employed with regard to psychological interventions for chronic pain (Turner \& Romano, 1984). Such interventions include operant conditioning (Fordyce, 1976, 1983), cognitive-behavioral 
approaches (Turk et al., 1983), and psycho-physiological methods (Gessel \& Alderman, 1978; Grzesiak, 1977) such as relaxation.

Finally, clinical pain assessment procedures can be classified according to the same three response systems approach (Keefe, 1982; Sanders, 1979b). Objective, observational measures are increasingly being used to assess gross motor pain behaviors (Fredericksen et al., 1978). The most frequently used measures, however, are based on self-report and focused on verbalcognitive aspects: pain intensity (Huskisson, 1983), temporal and qualitative characteristics of the pain experience (Melzack, 1975), and pain-coping strategies (Rosenstiel \& Keefe, 1983; Keefe \& Dolan, 1986). Measures for the physiological component include electromyography (EMG), skin conductance, and evoked potentials. These measures are difficult to apply in a clinical setting and their use has been limited to the laboratory (Flor et al., 1985).

The trichotomy described above is, in fact, similar to the three-systems model of emotions initially introduced by Lang $(1968,1978)$ and thus far almost exclusively applied to fear and anxiety. Recently, the three-systems model has been applied to the management of chronic insomnia (Espie \& Brooks, 1989) and hypochondrias (Warwick \& Salkovskis, 1990). As far as we know, this model has never been explicitly referred to in the pain literature. Based on (a) the analogies between anxiety, depression and pain described by Swanson, (b) the international recognition that pain is to be considered not just a sensory, but also an emotional experience, and (c) the implicit references to a three-dimensional conceptualization in the pain literature $e_{x}$ it is worth examining the applicability of the three-systems model of emotions as a heuristic framework in chronic pain research, assessment, and therapy. Quite rightly, Philips (1987) suggested that psychologists working on the relatively new and novel area of pain processing and pain control might benefit from the large and succesful work on fear. Theoretical notions that have been developed to explain pathological fears may prove useful in explaining the characteristics of chronic pain.

\subsection{The three-systems model of emotions}

In this section, the major elements of the concept 'emotion' are identified and their probable relations to each other are specified within the context of the three-systems model of emotions.

Within the behavior therapies, the three-systems model of emotions, featuring the concept of partially independent systems of emotional functioning, has, received considerable attention. According to this model 
emotions are thought to be action sets expressed by three different response systems: verbal report, overt motor responses, and expressive physiology (Hodgson \& Rachman, 1984; Hughdahl, 1981; Lang \& Lazovik, 1963; Öhman et al, 1978; Rachman, 1977, 1978, 1981). Emotions can, therefore, best be studied through these three response systems, by something which is referred to as a three-systems analysis. In the experimental work of Lang \& Lazovik (1963), which in fact gave birth to the formulation of this model, subjects with a snake phobia reacted in a very characteristic way to a systematic desensitization procedure. Some subjects showed a rapid decrease in overt behavior (decrease in phobic avoidance of the feared object) but insisted that they were just as anxious after treatment as before. However, later on, the cognitive response system would often 'catch up'. This cognitive lag has been noted in a variety of other studies (e.g. Borkovec 1972; Mathews et al, 1976). Other subjects showed a decrease in the selfreported level of anxiety yet appeared physiologically as aroused as before treatment.

Similar findings were later supported by Matthews (1978) and Schroeder \& Rich (1976), who concluded that changes in one system do not necessarily cause changes in the other two systems. In their research, response system changes occurred not only desynchronously but also idiosyncratically. Unfortunately, no follow-up measures were included in their study, so possible delayed covariance could not be examined. The conclusion was that anxiety can no longer be considered a sole entity that can be examined in three alternative ways. Rather, it should be considered as "a set of loosely coupled components..." (Rachman, 1978). The assumption is that anxiety can be generated by three different response systems or "channels' which act partially independently from one another.

In an attempt to describe the kind of relationship that exists among the three systems, Rachman \& Hodgson (1974) introduced the concepts of 'concordance' versus 'discordance', and 'synchrony' versus 'desynchrony". Concordance refers to the intercorrelation between any two of the three response systems. Discordance refers to the absence of high intercorrelations. Synchrony refers to covariance of change of the response systems over time. Desynchrony refers to the absence of such a covariance, illustrated both in Lang \& Lazovik's experiment, and in Schroeder \& Rich's research.

Hodgson \& Rachman (1974) also argued that concordance between responses systems was more likely to occur during strong emotional arousal. Conversely, discordance would be more evident when emotional responses were relatively mild. Their reasoning was that during high arousal, physiological reactions would probably be interpreted by the individual as causally related with the emotion. Low arousal, on the other hand, would not pro- 
mote such attributions. The latter assumption was not supported by Kaloupec \& Lewis (1983) and Lande (1982) who found, on the contrary, greater concordance during low arousal.

The model is complicated by the observation that desynchrony not only exists among the three response systems, but that it may also apply to variables within one response system as well. A study with height phobics by Abelson \& Curtis (1989) suggested that the physiological response system is not a single synchronized system but has multiple components which may change in different directions as change occurs in the verbal-cognitive and the overt-motoric systems.

The general framework of the three-systems model of emotions is illustrated in figure 2.1., which shows how emotion is inferred from a situational context in concert with information provided by the three response systems. This implies that emotions are best seen as hypothetical constructs, which are impossible to define exhaustively by any particular operation.

"As hypothetical constructs, 'emotions' have theoretical meaning above and beyond that inherent in any particular observation. Like other scientific constructs, such as "atom", they are not directly observable in themselves, but can be inferred only by their effects at

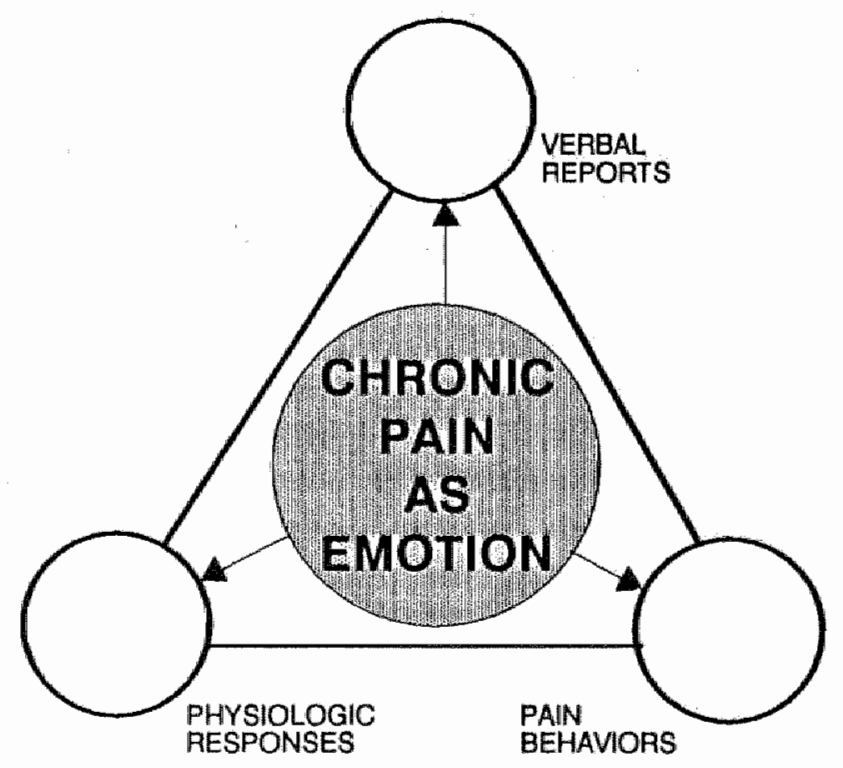

Figure 2.1. The inferential basis of chronic pain. Thick lines represent observable response systems and their relations. Arches represent inferred constructs (based on Öhman, 1987). 
some observable level. Thus, emotions are indicated by a range of observable events, none of which have an unchallenged status as a necessary or sufficient condition. In particular, the everyday practice of taking emotions to be isomorphic to phenomenal experience, or verbal reports of such experience, must be firmly rejected in a scientific analysis [italics added]." (Öhman, 1987, p. 82).

This view is consistent with the proposition of Lazarus et al. (1970) that emotions can best be understood as response 'syndromes', analogous to the diagnosis of medical disease syndromes:

"An emotion, like a disease, is not defined by any single symptom or set of symptoms, nor has it one center or locus (e.g., in the hypothalamus). For example, subjective experience may serve an important function in emotion, bridging informational gaps for the individual ... and providing diagnostic signs for assessment by others (e.g., in self-reports). But subjective experience cannot define emotion any more than it can define disease. Thus, headache is an important diagnostic symptom of brain tumor, yet many brain tumors do not produce headache and many headaches do not indicate brain tumor. Similar considerations apply to physiological changes during emotion ... and to behavioral manifestations. Although a disease syndrome is not defined by any single sign or symptom, or any fixed set of symptoms, it remains an extremely important concept. Indeed, a whole branch of medical science, nosology, is devoted to the identification and classification of disease syndromes. A similar nosology for emotional phenomena is needed in psychology. (p. 211)"

Some emotion theorists have pronounced divergent views upon the sequence in which the three response systems occur in the generation of emotions. The major protagonists in this debate are Zajonc $(1980,1984)$ and Lazarus (1984). In fact, this debate is not new at all. More then a century ago, in the 1880's, the eminent American psychologist William James, and the Danish psychologist Carl Lange independently presented similar theories of emotion. Their doctrine is opposed to the common sense view that emotions are the result of the evaluation or the appraisal of the stimulus. The differences are well expressed by James (1890):

"Common-sense says, we lose our fortune, are sorry and weep; we meet a bear, are frightened and run; we are insulted by a rival, are angry and strike ... My theory ... is that the bodily changes follow 
directly the perception of the existing fact, and that our feeling of the same changes as they occur is the emotion ... that we feel sorry because we cry, angry because we strike, afraid because we tremble; and not that we cry, or tremble, because we are sorry, angry, or fearful, as the case may be." (cited in Krech et al., 1974)

The James-Lange theory thus asserts that when an emotion-provoking stimulus is presented, we first react with the muscular and glandular responses. It is only when the feedback from these responses arrives at the cortex that we feel or experience the emotion. In the current debate, a similar view is shared by Zajone $(1980,1984)$ who suggested that the physiological response is immediately and automatically activated by the stimulus, preceding slower and more deliberate cognitive inferences (primacy of affect). Another influential proponent of this view is Schachter (Schachter \& Singer, 1962).

The alternative view is put forward in Lazarus' cognitive theory of emotion (Lazarus, 1970, 1984). He postulates that an emotion results from a series of cognitive appraisals of environmental events in a continuous sequence between 'primary appraisal' ("What is the significance of the stimulus?"), 'secundary appraisal" ("What can I do about it?"), and 'reappraisal' ("What effects do my actions have?"). The outcome of this sequence of appraisals determines emotional, and as part of $i t_{,}$physiological activation (primacy of cognition).

The debate on primacy of affect or cognition is hampered by a lack of sound definitions of what is cognitive and what is not cognitive. But even when such definitions are provided, we share Plutchik's (1985) view that the debate on the issue of primacy is not a fruitful one. An emotion can best be defined as a chain of events that has certain loosely coupled elements in a complex feedback loop system. The chain contains the elements of cognition, physiological arousal, and motoric behavior, is preceded by a specific environmental or internal stimulus, and is followed by certain consequences. From this point of view it is irrelevant to speak of primacies:

"When emotions are conceptualized as complex chains of events with feedback loops, it is obviously possible to focus attention on any of the elements of the chain. One can then produce theories that emphasize, for example, the primacy of arousal, or the primacy of expressive behavior." (Plutchik, 1985, p. 199)

The three-systems model of emotions has distinct consequences for the assessment and treatment of so-called pathological emotions. These conse- 
quences will be adressed later in this chapter. In the next section, the applicability of the three-systems model of emotions to chronic pain will be examined.

\subsection{The three response systems of chronic pain}

\subsubsection{The overt-motoric response system}

The overt-motoric system covers gross motor behaviors, referred to in pain research as pain behaviors. Fordyce $(1968,1976,1983)$ particularly demonstrated the clinical and theoretical relevance of observable pain behaviors. Chapter 3 is devoted to an empirical examination of the dimensions and components of overt pain behavior. The most commonly drawn conclusion is that overt pain behavior is a multi-dimensional construct. The study in chapter 3 revealed three dimensions that characterize the overt pain behavior: "withdrawal-approach", "high arousal-low arousal" and "visible behaviors-audible behaviors".

What is the working mechanism within this overt-motoric response system? Fordyce not only introduced the concept of pain behavior, he also introduced the basic conditioning concepts in chronic pain and described the operant conditioning paradigm (originated by Skinner, 1953) in particular. Pain behavior is considered to be under consequent control. When pain behavior is expressed by a patient, good things can happen (positive reinforcement) and unpleasant events can be avoided (negative reinforcement). By means of these environmental contingencies, pain behavior, and the chronic pain problem, can be maintained long after healing has occurred. Many investigators have demonstrated that pain behaviors can be increased through systematic positive reinforcement (Cairns \& Pasino, 1977; Block et al., 1980, Ford yce et al., 1973; White \& Sanders, 1985).

When the emittance of a certain pain behavior leads to the possibility to avoid unpleasant events, the probability of occurrence of this behavior will be strenghtened. In elderly people, for example, pain behaviors can be negatively reinforced when they lead to the avoidance of responsibilities or situations which have become difficult to cope with because of cognitive deficits. In a case study described by Cosyns \& Vlaeyen (1983) pain behavior was maintained because marital conflicts could be avoided.

In order to specify reinforcing events, behavioral theorists have distinguished among enduring setting events, temporary setting events, and pinpointed events:

"The end uring setting events represent the more permanent and more inclusive collection of events. Ongoing activities can be regarded as 
a more temporary subset of interrelated events, and pinpointed events may be regarded as a highly specific subset within some temporary activity" (Moxley, 1982. p. 48).

These distinctions point to the variety of sources for operant control that exists in the natural environment. These are not limited to consequences that immediately follow pain behavior. In laboratory experiments only pinpointed events can be manipulated, thereby complicating the empirical validation of the operant paradigm in chronic pain. In the case study presented by Cosyns \& Vlaeyen, pain behaviors were assumed to be maintained not by pinpointed events, but by a longstanding, enduring setting event being the marital conflict.

The terms "secondary gain" and "tertiary gain", are sometimes used in this context. Secondary gain refers to the positive consequences that the pain patients experiences when pain behaviors are displayed and which otherwise would not occur. Tertiary gain refers to the positive consequences that the spouse or other family members experience when the patient has a pain problem. Tertiary gain may hamper the positive reinforcement of healthy behaviors by these family members during treatment.

As an extension of the heuristics of the three-systems model, Lethem et al. (1983) introduced a fear-avoidance model applicable to the cases of chronic pain in which a discrepancy is observed between the objective physical findings and pain behaviors. The central concept of their model is fear of pain and consequent pain avoidance. When in an acute pain situation, two kinds of responses are hypothesized: "avoidance" and "confrontation" (=approach). Avoidance of pain-eliciting (and thus fear-eliciting) activities leads to a diminishing repertoire of physical and social activities. This results in a lack of exposure to sensory stimuli, fewer opportunities to test the reality of pain experienced, and an increased likelihood of the occurrence of reinforcing consequences. Extreme behavioral avoidance in the long run can lead to a number of disabling physical consequences (loss of mobility, muscle strenght, and fittness) possibly resulting in the "disuse syndrome" (Bortz, 1984), and psychological consequences (depression, loss of self-esteem, and pain focusing). In the case of approach, however, the individual can resume an increasing range of physical and social activities, possibly resulting in fear reduction.

The reasons that have been hypothesized in relation to why some pain patients avoid and others do not avoid physical and social activities when experiencing pain can be summarized as follows:

1. According to the model of Lethem et al. (1983), chronic pain patients 
may limit activity because of fear of anticipated nociceptive stimuli and consequent pain. Moreover, cues other than pain can, through the process of stimulus generalization, become capable of eliciting avoidance behaviors. These cues may be internal (e.g. proprioceptive information, such as fatigue) or external (e.g. certain demands or responsibilities).

2. Avoidance may be related to cognitive factors. Proprioceptive signals that accompany activity may be misinterpreted as a consequence of which a pain patient may decide to stop the activity (Schmidt, 1986). Recent research in both experimentally induced pain and dental pain shows that when inaccurate predictions of pain lead to unpleasant disconfirmations, subsequent avoidance behavior increases as does pain report (Arntz, 1989; Arntz et al., 1990). Pain intensity, however, is shown not to be related to actual activity levels (Linton, 1985). Dolce et al (1986) showed that tolerance time (approach) of chronic pain sufferers did not correlate with current pain ratings, but with self-efficacy expectancy ratings.

3. For people having experienced stressful life-events, avoidance of activities also means reduction of responsibilities and life stresses. In the acute phase the avoidance behaviors are considered pain-contingent. Avoidance is effective in reducing pain or suffering from nociception. As the pain problem persists; the patient may learn that suffering, due to experiences other than pain, can be reduced through the same avoidance behaviors.

4. Personality characteristics may influence the probability by which avoidance may occur. Pain patients who are concerned about physical health, or patients who like to be the centre of attention might experience essential reinforcers when avoiding activity.

5. A poor physical condition, as a consequence of avoidance, may in turn be a reason to further avoid activities. However, avoidance cannot always be explained by poorer physical condition (Schmidt, 1986).

\subsubsection{The physiological response system}

So far, little is known about the physiological response system of chronic pain. The yet unanswered question is: What are the parameters we are looking for, and which are relevant to the chronic pain syndrome?

Emotions are said to be initiated by the so-called "orienting reflex" (OR). The OR consists of an unspecific ensemble of responses elicited by novel or significant environmental stimuli (Maltzman, 1987). The OR can 
be observed through the physiological response system and can, through classical conditioning, be elicited by previously "neutral" stimuli. The OR is considered that part of the emotion that is responsible for the elicitation and initiation of emotions.

We are not aware of studies which have examined the relationship between the OR and chronic pain. The persistence of the OR-related physiological responses, when the external stimulus is over, is what appears to happen with chronic pain. Initially, increased nociception might serve as the OR eliciting stimulus. Later on, bodily sensations, initially accompanying nociception, or even events in the environment, can elicit the OR and the physiological arousal by means of classical conditioning, thus maintaining the pain.

In contrast to psycho-physiological research on fear and anxiety, in which cardiovascular responses (such as heart rate) are the focus of attention, psycho-physiological research on chronic pain has been mainly focused on muscular responses through electromyography (EMG). Although EMG activity in back pain patients has been studied since 1966 (e.g. Arroyo, 1966; DeVries, 1966), only a brief review of the most recent studies is given. For a more extensive review, the reader is referred to Dolce \& Raczynski, 1985).

Kravitz et al., (1981) compared levels of paralumbal muscle activity for chronic low back pain (CLBP) patients and normal subjects during periods of rest and voluntary muscle contraction. Although the resting state EMG measure did not differ in the two groups, the CLBP patients exhibited higher mean levels than the non-pain group during muscle contraction. Collins et al., (1982) examined physiological arousal levels in CLBP by measuring paraspinal and frontalis EMG, heart rate and skin resistance during movement. In contrast to the previous study, the CLBP group showed similar or significantly less paraspinal muscle activity than the control group. Frontalis EMG and skin conductance were higher in the CLBP group. These elevations occurred both in the resting state and with the laboratory induced stressors. Unfortunately, the chosen stressors were not checked against personal relevance. Cram and Steger (1983) compared EMG activity in four diagnostic groups: headache only, neck/shoulder/upper back only, low back pain only and mixed pain states. The authors found that elevated bilateral levels of frontalis and masseter EMG distinguished headache patients, while the discrepancy between right and left paraspinal EMG distinguished the CLBP group. As an extension of Collins et al.'s work, Flor et al., (1985) investigated the relationship between paraspinal EMG and personally relevant stress situations in three groups: a chronic back pain (CBP) group, hospital patients without back pain, and subjects with- 
out pain or any other complaints. The physiological assessment (paraspinal and frontalis $\mathrm{EMG}$, heart frequency and skin resistance) took place under four conditions: discussion of personally relevant stress, talk about pain, mental arithmetic and recitation of the alphabet. The results indicated that the CBP group displayed elevations and delayed return to baseline only in their paravertebral musculature and only when discussing personal stress. Furthermore, the results were more pronounced in the left paraspinal EMG than in the right. This finding supports the assumption that emotions are basically processed by the right cerebral hemisphere and thus expressed more clearly in the left side of the body (Tucker, 1981). In a more recent review, Flor \& Turk (1989) concluded that psycho-physiological responses are not only stress-related, but symptom-specific as well.

In sum, ample research is needed to reveal the relevant parameters representing the physiological response system in chronic pain. Results of present research suggest that these parameters may be different in different pain locus groups. For headache, frontalis EMG might be relevant (Bakal et al., 1984). For CBP, left paraspinal EMG appears to be relevant (Flor et al., 1985). Not only is the elevation important, but the delayed return to baseline and the condition in which the assessment takes place also appear to be important.

Can the operant and respondent learning paradigms be applied to the physiological response system? Three general etiological approaches have been described that account for what is called 'myogenic back pain': the physical stressor model, the psychosocial stressor model, and the biomechanical model.

The physical stressor model argues that some physical stress (trauma or structural damage) to the musculoskeletal system results in reflexive and protective muscle spasm and its associated pain. Pain and muscular spasm occur together as the result of some physical pathology. Pain caused by the initial spasms may act as an additional stressor leading to further muscular tensing. This then elicits more pain until finally a pain-spasm-pain cycle has been established (Simons, 1976).

The psychosocial stressor model is the second muscular tension model of back pain. This model also argues that back pain can result from increased paraspinal muscle activity. However, the increased tension is believed to result from ineffective coping with environmental and emotional stress. In this context the disorder 'tension myositis' has been introduced by Sarno (1978), referring to the process during which increased psycho-social stress results in increasing muscle tension and associated pain.

The biomechanical model proposes that faulty patterns of muscular activity adversely affect the biomechanics of natural movement and result 
in mechanically induced pain. This model differs from the previous models in the believe that abnormally low levels of paraspinal muscle tension or muscular asymmetries lead to back pain through biomechanical mechanisms rather than increased muscle tension (e.g. Wolf et al, 1982).

These models may not necessarily be mutually exclusive. In our view the models are valuable at different times within the transfer from acute to chronic back pain states. The classical conditioning paradigm provides the link between the physical and psychosocial stress models. Suppose that, in accordance with the biomechanical model, nociception occurs as the result of nerve root irritation. Following the physical stress model, an acute pain state is associated with both nociception and muscle tension. Nociception is the unconditioned stimulus (US) and muscle tension the unconditioned response (UR). Stimuli (both internal as external) associated with the original nociception can, through classical conditioning, become conditioned stimuli (CS) capable of eliciting tension as the yet conditioned response (CR). Through the process of stimulus generalization, other stimuli associated with pain and nociception act as a CS (Gentry and Bernal, 1977). In this phase, the psycho-social model can be applied. Empirical support for the psycho-social model can be found in the studies of Collins et al. (1982) and Flor et al. (1985).

Physiological arousal can also be intensified by conditioned fear of movement, leading to avoidance of activities and mobility (Lethem et al., 1983). Although there is some evidence that autonomic and physiological reactions can be conditioned in an operant way (Miller, 1969), it is generally assumed that they are more sensitive to classical conditioning (van Olst et al., 1980).

In this context, the concept of "respondent pain" needs clarification. In his exposition on the role of learning principles in the maintenance of pain, Fordyce (1976) distinguishes operant pain from respondent pain. Operant pain is said to be under the control of social contingencies, while respondent pain is said to be the result of antecendent nociceptive stimuli related to tissue damage. From the three-systems model perspective, respondent pain (i.e. chronic pain which came about by classical conditioning) can exist without any tissue damage.

\subsubsection{The verbal-cognitive response system}

In the literature of the last decade, the role of cognitive mediational processes in the regulation of pain is emphasized (Tan, 1982; Turk et al., 1983). Paradoxically, it is hard to find any sound definition at all of cognition. Several attempts to conceptualize this verbal-cognitive system have led to problems similar to those regarding anxiety, problems which have resulted 
in unresolved scientific discussions. Hugdahl (1981) examines the applicability of three alternative conceptualizations of cognition; cognitive labeling of physiological arousal, anticipation of a stressful event by worrying, and thinking style characterized by the internal dialogue or self-talk.

Kendall and Korgeski (1979) distinguish cognitive content (beliefs, attributions, and self-efficacy) from methods for assessing cognitive phenomena (in vivo thought sampling, self-statements, and imagery). The clinical relevance of cognitions is said to be related to their differential accessibility. Beliefs are considered to be difficult for patients to report, mainly because of a lack of awareness. In addition to paradigm-relatedness, Kendall \& Korgeski (1979) underscore the importance of choosing (causal) attributions and (self-efficacy) expectations as relevant and accessible cognitive processes.

Yet the question remains: what has to be understood by pain cognitions? The focus is directed towards the pain experience or how people think about their pain. Many authors refer to them as covert pain behaviors. Some authors apparently assume that every reader knows the meaning of the term and they do not give any definition (Harris \& Rollman, 1985; Spanos et al., 1975). Most authors do, however, give some examples to illustrate the meaning of cognition, among them "thoughts, feelings and images" (Sanders, 1979a), "things inside the head, private events" (Jaremko, 1978), "expectancies and self-statements" (Hackett \& Horan, 1980), "attitudes, beliefs, expectations" (Turner \& Chapman, 1982), "appraisal of the environment" expectancies and ideas" (Tan, 1982), and "information processing" (McCaul \& Mallot, 1984).

It is clear that further research is needed to establish a uniform definition of the verbal-cognitive response system of pain and one that is most likely probably not limited to pain. Cognitively oriented emotion theorists such as Lazarus (1979) and Bandura (1977) have introduced cognitive concepts that have recieved widespread acceptance.

After the OR has redirected the attention of the individual, another process, referred to as 'primary appraisal" (Lazarus et al., 1979) is hypothesized. Primary appraisal refers to the cognitive proces of analyzing the stimulus to further assess its relevance or potential danger. In contrast to the OR, primary appraisal would be slower and more deliberate. Primary appraisal concerns the question asked: "Am I okay or in trouble?", and the answer lays in the meaning or the interpretation of the stimulus to the individual. These interpretations are also called 'causal attributions'. A causal attribution answers a 'why' question such as "Why do I (still) have pain?". Although there are numerous possible attributions, they can all be characterized by three dimensions; locus (whether the cause is located 
inside or outside the person), stability (whether the cause is lasting or not), and controllability (whether the cause is subject to volitional control or not) (Weiner \& Graham, 1984).

Beecher (1956) was one of the first who illustrated the effect of the meaning attached to a pain. In a comparative study of (acute) wound pain, a group of male civilian patients undergoing major surgery was compared to a group of wounded soldiers. Of the soldiers, about one-third wanted medication to relieve their pain and two-thirds did not. Of the civilians, suffering from far less tissue trauma, four-fifth requested analgesics and one-fifth did not. As Beecher (1959, p. 165) stated:

"The important difference in the two groups seemed to lie in their responses to the wounds. In the wounded soldier it was relief, thankfulness for his escape alive from the battlefield, even euphoria (his wound was a good thing). To the civilian his major surgery, even though essential, was a depressing, calamitous event."

Spanos et al. (1971) reported that subjects who display catastrophizing attributions (presumably externally localized, unstable, and uncontrollable) reported higher pain levels to a cold pressor task than non-catastrophizing subjects. Leventhal et al. (1979) showed how negative affect can be changed by the way the stimulus is labeled. Announcement of a form of arousal resulted in more intense pain experience and subjective report compared to the condition in which subjects received sensory information. Lefebvre (1981) studied cognitive errors in depressed and non depressed low back pain patients using the General and Low Back Pain Cognitive Error Questionnaire (CEQ). Cognitive errors are in this study defined as distorted or illogical inferences based on available information. Empirical categorization of typical causal attributions used by different chronic pain patients is still to be done.

After the interpretation or primary appraisal of the stimulus is made, the individual will, based on these causal inferences, try to reduce his suffering. However, before action can be undertaken, the person must be aware of the possible alternatives (if they exist) among which to choose. This process of evaluating an encounter with respect to coping resources and options is what Lazarus calls 'secondary appraisal'. Secondary appraisal gives an answer to the question: "What can I do about the pain?". Bandura introduced the concept of "outcome expectations" which has a similar meaning. Outcome expectations consist of beliefs about whether a given behavior will lead to given outcomes. However, the presence of certain resources does not mean that the appropriate resource will be available or used at the time. 
There will often be occasions when the required skills are simply not mastered, or when some psychological deficit will prevent effective use of the resource. An example is that a pain patient may believe that exercise in general is a good thing to do, but not in his / her particular case because of the immediate negative consequences (e.g. increased pain).

This leads us to a third cognitive process which is referred to by Lazarus as 'reappraisal' and by Bandura as 'self-efficacy' or 'efficacy expectations'. Reappraisal provides an answer to the question "What effects do my actions have?", and occurs as new information is obtained from perceived changes in the environment. A self-efficacy expectation is defined as a personal conviction that one can successfully perform certain required behaviors in a given situation or, in the case of pain, that one will find an answer to the question: Am I effective in dealing with my pain or not? That self-efficacy expectations might play an important role in health management is illustrated by O'Leary (1985).

Dolce et al. (1986a) examined the effect of an exercise quota system on self-efficacy in chronic pain patients. The results demonstrate the efficacy of exercise quota systems to reverse not only patterns of inactivity, but also faulty beliefs related to avoid ance. The quota system, based on the operant conditioning principle, was shown to increase self-efficacy expectations. In another study (Dolce et al, 1986b) the same authors examined the role of self-efficacy expectations as outcome predictors for chronic pain patients. Although post-treatment self-efficacy was observed to significantly correlate with medication use, exercise levels and work status at follow-up, it failed to consistently account for a significant amount of the variance when other variables are considered. Of interest in this study is the relatively low correlation among post-treatment efficacy ratings and behavioral measures of exercise tolerance and medication use. This finding appears to indicate desynchrony among the verbal-cognitive and the overt-motoric response systems of pain. The authors, however, suggested that behavioral improvements will have little impact on self-efficacy expectations if they are attributed to external factors (such as the therapists) rather than personal skills or abilities. An additional cognitive therapy, focusing on the importance of the role of the patient's own possibilities to control pain might be expected to further increase self-efficacy.

Although the self-efficacy theory has received widespread acceptance, it has not gone uncriticized (Wilson, 1984, Lee, 1989). Lee (1989), for example, argued that the theory has strenghts as a metaphor for describing human behavior. Its weakness, however, lies in its failure to explain behavior. The problem is that expectations, like other cognitive processes, are only accessible to the scientist through self-report. It is well known that self-report is 
influenced by a host of distorting factors, such as social desirability, underand over-reporting. Moreover, cognitivists start from the assumption that behavior is the result of conscious processes that can be made verbal through self-report. Nisbett \& Wilson (1977) have made an argument that cognitive mediating processes are not detectable by mere introspection. As Taylor and Fiske (1978, p. 283) state:

"[Attribution processes] seem to occur automatically and substantially without awareness, and as such, they differ qualitatively from the intentional, conscious, controlled kind of search which we like to think characterizes all our behaviour ${ }^{\prime \prime}$

The same might also be true for expectations. Therefore, Lee proposes to consider expectations of any kind reflections of motoric behavior, not as causes.

One of the major shortcomings of cognitive therapy is the lack of a satisfactory theoretical framework (Wilson, 1982). What is worse, there seems to be no widely accepted paradigm covering the verbal-cognitive response system. The principle of parsimony (Feigle, 1956) holds that an explanatory theory should be based on the smallest possible number of basic concepts. The history of science suggests that knowledge accumulates most rapidly when the investigations of different scientists can be integrated by their relationship to a shared paradigm (Kuhn, 1962). A paradigm involves more than a theory. A scientific paradigm provides an organizational focus around which, by concentrating on certain key experimental situations, a body of related and integrated knowledge and a methodology of investigation and measurement can be developed. Taking this principle into account, one might examine the applicability of both the operant and respondent conditioning paradigms to the verbal-cognitive response system. Although lenghty discussion of this topic is outside the scope of this study, we will briefly describe such a model. The assumption then would be that the application of these paradigms results in two theories: the attribution theory (Weiner \& Graham, 1984) and Bandura's self-efficacy theory (Bandura, 1981; Dolce, 1987) (see Figure 2.2).

The attributional process, or providing a signal value to a set of external and internal stimuli is, according to the contiguity principle, controlled by antecedent factors. A link between attribution theory and classical conditioning has been described in more detail by Eelen (1982).

Self-efficacy expectations are believed to be controlled by four main sources of information: performance accomplishments, vicarious experiences, verbal persuasion, and emotional arousal. Mastery experiences gained 
through performance accomplishments are hypothesized to have the greatest impact on self-efficacy expectations because they provide the most information about actual capabilities. Therefore, self-efficacy expectations seem to be mainly under consequent control. Woods (1985), showing the feasability and value of analyzing complex human behavior into basic instrumental conditioning paradigms, proposed to expand a taxonomy of operant conditioning so as to include prior expectations regarding the consequences of such behavior. He argued that if, for example, an individual expects his behavior to lead to a reward, and it does, then the probability of maintaining both the expectacion and the behavior is increased. Woods" scheme is an interesting and potentially useful merging of a cognitive perspective with a traditionally non-cognitive behavioral approach.

As both classical and operant conditioning processes occur predominantly outside awareness, it is very likely that the forming of attributions and expectations also lie in the field of unconscious cognitions. An important consequence is that researchers who solely rely on clients introspections of their cognitions limit their efforts to those cognitions that the individual is aware of and is able to report. Nevertheless, both the attribution theory and the self-efficacy theory are helpful to provide new labels that help us conceptualize the verbal-cognitive response system of pain. The question remains whether they really add novel rules and new scientific content.

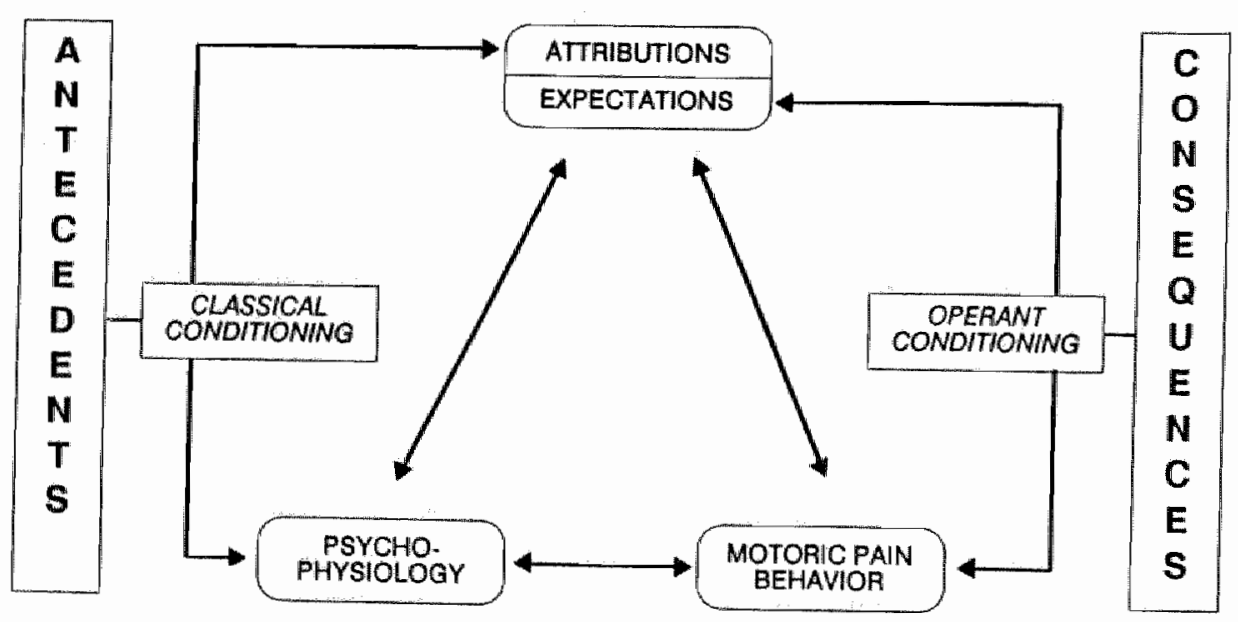

Figure 2.2. A hypothetical model in which both the classical and operant conditioning paradigm are applied to the three response systems of pain. 


\subsection{Concordance or discordance?}

Gannon \& Haynes (1986) emphasize the importance of discordance in the etiology of psychophysiological disorders. Based on a series of studies, they conclude that discordance between the cognitive and physiological response systems in patients with headaches, sexual dysfunctions, and insomnia is higher than in people not suffering from these complaints. Syrjala and Chapman (1984) consider the lack of congruence between behavioral and self-report measures a fact, and argue in favor of an increased use of behavioral measures. Several studies have demonstrated significant discrepancies between what pain patients report they do and what is actually observed (e.g. Kremer et al., 1981; Sanders, 1983).

A systematic study of the relationship between the three response systems in chronic pain has not yet been carried out. There are no studies available that provide data on the concordance between the motoric and the physiological system. Correlations between measures representing the three response systems of chronic pain are calculated but in different contexts. We will review some of them to illustrate that in chronic pain, as in anxiety, discordance exists. Table 2.I summarizes the results of this review.

Several authors reported that most clinicians find little concordance between non-verbal pain behavior and pain ratings. Discrepancies between nurses' observations and self-reported pain measures were more pronounced in chronic pain patients than in acute pain patients (Teske et al., 1983). In an attempt to validate their UAB-Pain Behavior Scale, Richards et al. (1982) correlated the results of the scale with results of the visual analog scale. Correlations were positive but not significant. By contrast, Keefe \& Block (1982) report significant positive correlations between self-report and carefully made observations: Concordance is found instead of discordance. One reason may be that in this study measures were taken in a laboratory setting where the subjects might have tried to deliberately let their verbal responses correspond with their actual behavior.

In a recent study by Romano et al. (1988), who used the Keefe \& Block (1982) pain observation protocol, only moderate correlations were found between pain behaviors and pain intensity scores. Correlations between observed pain behaviors and VAS ratings of average pain over the previous week were inexistent. These findings are in accordance with those of Kleinke \& Spangler (1988) who found low correlations between observed pain behavior and McGill Pain Questionnaire scores. Of interest is that the correlations were higher when measures were obtained at the end of a multimodal pain management program ( $\mathrm{r}$ ranging between $0.25-0.33$ ) as compared to the start of the program ( $\mathrm{r}$ ranging between $0.08-0.24$ ). 
The relation between back pain and paraspinal EMG is found to be far from unequivocal (Dolce \& Raczynski, 1985). Some studies have examined the relationship between pain report and EMG. Data are substracted from studies evaluating the effect of EMG biofeedback. Keefe et al. (1981) found that subjective ratings of pain and tension were significantly correlated but EMG was not significantly correlated with pain or subjective tension. On the other hand, Large \& Lamb (1983) found a positive and significant correlation between pre-session pain ratings and pre-session EMG for each session.

From the three-systems model of emotions perspective that the three systems are only "loosely coupled", it is expected that positive but low correlations will be found between measures representing different response systems. In the case in which plenty of overt pain behaviors are maintained through operant conditioning, pain problems should not be expected to correlate strongly with abnormal EMG levels.

A question that remains is whether the low correlations found are not due to 'apparent $t^{t}$ discordance, discordance that is attributable to methodological errors. One frequently noted methodological source of discordance is method variance. The level of apparent discordance is increased when different methods (e.g. self-report vs. observation) are used to measure the same construct. A thorough discussion of this and other errors is given by Gannon \& Haynes (1986).

\subsection{Synchrony or desynchrony?}

Only few studies have examined synchrony and desynchrony among the response systems during a pain treatment program. One of the main reasons is probably the lack of a multiple response assessment in clinical evaluation research. One study that is worth citing, however, is the component analysis of a multi-modal treatment program done by Sanders (1983). Unfortunately, very few subjects were involved. The treatment components covered all three systems (cognitive restructuring, relaxation, social reinforcement of activities) and also included assertiveness training. The employed assessment techniques covers only two systems (self-report and observation). Although no correlations were calculated, the graphical representation of the course of the measures during treatment suggests synchrony. This finding suggests that synchrony is reached when the treatment focuses on all three pain response systems. More research is needed, however, to determine whether changes in one system will lead to changes in the two other systems and whether clinically meaningful differences exist between pain patients 


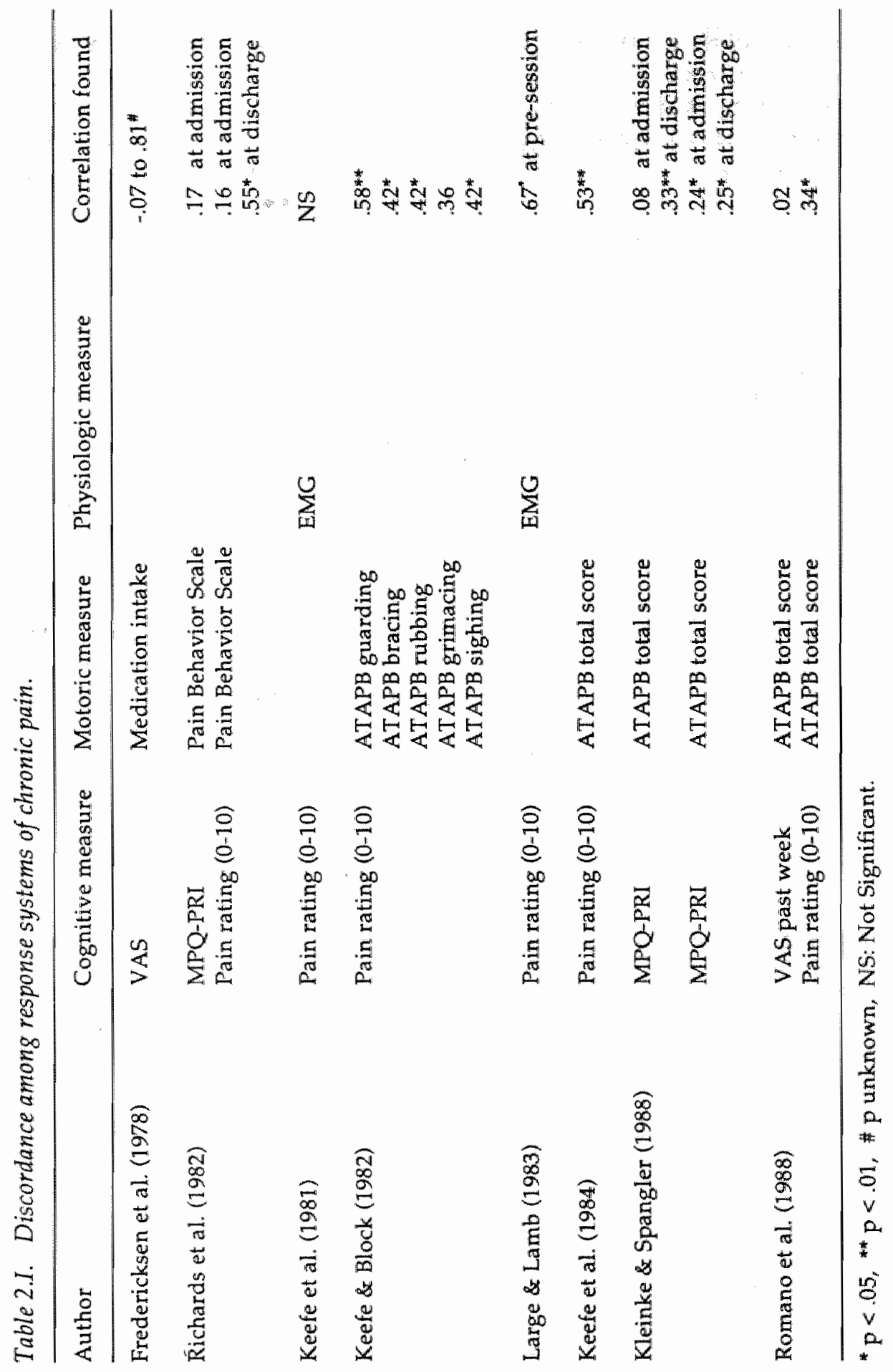


who do and those who do not demonstrate desynchronous responses to a certain treatment modality.

Kincey \& Benjamin (1984) examined the relationship of measures of subjective pain experience, negative mood state and activity levels of severely disabled chronic pain patients receiving an operant treatment program. Prior to treatment, there was a high positive correlation between pain intensity and negative mood state, and a negative correlation between pain intensity and levels of activity. Following treatment both these correlations were reduced. Only the correlation of activity and mood state showed little change. The data showed that although activity levels increased significantly for almost all patients, pain intensity levels decreased for only $50 \%$ of the subjects. Desynchrony occurred, probably because the treatment was focused only on the overt-motoric dimension of the pain, and not on the verbal-cognitive.

Romano et al. (1988) examined the extend to which overt pain behaviors change following an inpatient multidisciplinary treatment program, and whether such differences are associated with changes in self-reported pain behaviors, depression and pain. Although they found significant decreases in pain behaviors and self-report measures, the decreases in observed pain were significantly associated with decreases in depression $(r=0.48, p<0.01)$, but not with changes in self-reported pain or self-reported pain behaviors. Although different measures were used, these findings are in accordance with the results of Kincey \& Benjamin, and also suggest desynchrony.

Desynchrony between the cognitive and physiological response system is shown by Nouwen (1983) who was able to decrease EMG levels with EMG biofeedback, but the reported pain did not decrease.

\subsection{Consequences for chronic pain assessment}

Describing pain as a subjective feeling accompanied by physiologic changes and instrumental behaviors points to an enduring dilemma: On the one hand, pain would only be accessible for observation by the person concerned. On the other hand, the transformation of pain into an objective status as verbal reports is fraught with difficulties. One potential trap in the enterprise of relying only on subjective pain report is the notion that subjects have the potential for reporting something that might be defined as "true" pain. This has been referred to in classical measurement theory as the "Platonic true score". When we think of pain as a construct, such as anxiety and depression, we must recognize that it is best measured in terms of the dimensions that it is supposed to affect, only one of which is subjective report. In agreement with Lang's (1968) caution about emotional responses 
as loosely related systems, it is a mistaken notion that self-ratings of pain and observations of pain behavior can be used substitutively. Self-report questionnaires have received the most attention in the past, probably because the other forms of assessment are more difficult to carry out in a clinical setting. Furthermore, given the possible discordance among the systems, concurrent validity of the assessment instruments can only be established with measures chosen from the response system to which the validated instrument belongs.

The assumptions that the three response systems relate to each other in a discordlant way and that measures of the three systems probably change idiosyncratically lead to a model in which a chronic pain patient can be ideally characterized as a motoric, cognitive, or physiological pain patient (or combinations). Indeed, a patient can have high or low scores on each system. In order to empirically support this assumption and to be able to determine a patient's score, adequate, reliable, and valid measuring instruments are needed for the systems: observation scales for the motoric system, self-report questionnaires for the cognitive system, and apparatus for the physiological system.

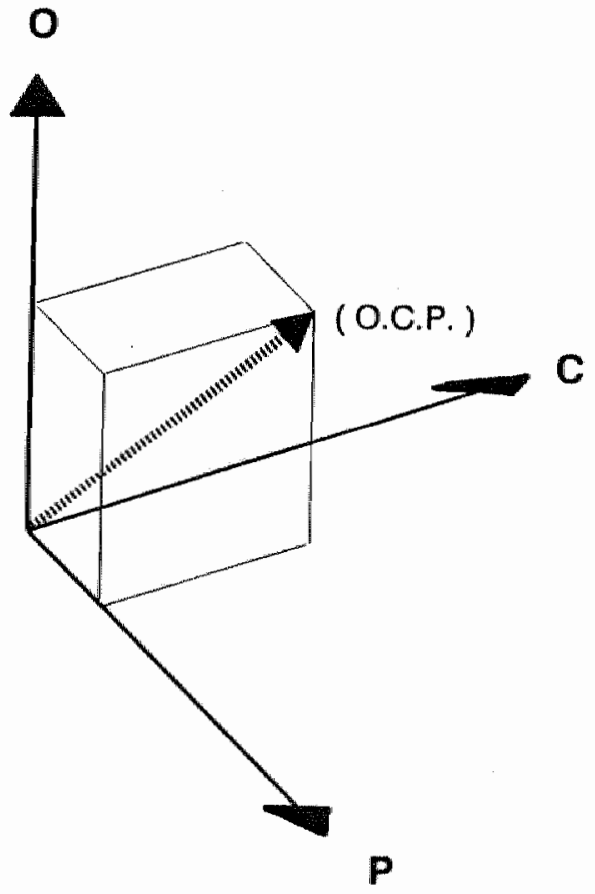

Figure 2.3. Chronic pain represented as a vector in a three-dimensonal space. $\mathrm{O}=$ overtmotoric response system, $C=$ verbal-cognitive response system, $P=$ physiological response system. 
We agree with Syrjala and Chapman (1984) that a pain profile similar to the Minnesota Multiphasic Personality Inventory (MMPI) would be ideal. Such a profile should be based upon observational, self-report and physiological data, possibly expressed in T-scores. The next step would be to represent chronic pain as a vector in a three-dimensional space (Figure 2.3.). A similar representation has been proposed by Van Egeren (1978) for fear and anxiety. The length of the vector would be the resultant of the portion of the three systems and a reasonable measure for the gamut of the chronic pain problem.

At least three obstacles need to be surmounted in order for one to be able to create such a multi-system profile. First, clarity and a consensus are needed with regard to the conceptualization of the three systems in chronic pain. Second, for each of the three systems, reliable and valid measurement instruments need to be developed. Finally, comparable standard scores need to be created so that the relative disturbance of the three systems can be calculated for each pain patient.

Such a three-systems analysis would focus on the pain complaint as such and tell nothing about possible physical findings, psychosocial information or other complaints in which the pain problem can be embedded. A complete assessment, however, should also include these. Recently, Turk \& Rudy (1987) have developed such a broader assessment system in which physical, psychosocial and behavioral information is integrated.

The presence of desynchrony among different pain measures implies that clinical treatment programmes will need to evaluate the effectiveness of their component parts against different criteria in order to establish which components, if any, are effective on which measures.

\subsection{Consequences for chronic pain treatment}

If a three-systems analysis were to reveal that, for each chronic pain patient, one of the three systems is the most "disturbed", would the treatment then need to be chosen according to the results of this analysis and to the abovementioned profile? In anxiety research, a similar question is answered positively (Hugdahl, 1981; Rachman, 1981). The assumption is that the type of treatment that focuses on the main response system would prove to be the most successful. This implies that treatment focused on modifying overt pain behaviors will be the most successful for "motoric" pain patients, a cognitive treatment the most successful for "cognitive" pain patients, and a respondent treatment, such as relaxation or systematic desensitization the most successful for "psycho-physiologic" patients. 
What are these treatments? Generally, treatments can be defined according to their pursued effects, or according to their point of application. The first criterion probably is too vage. All therapies which incorporate some kind of successful performance will result in cognitive changes. This would mean that almost every treatment can be called cognitive. In order to be able to evaluate the relative effects of various treatments, they must be defined more sharply. The answer to the question: "what is the mechanism I want to interfere in?" more clearly delineates different treatments. According to the three-systems model of pain, treatments can aim at the modification of each response system. Motoric treatments are aimed at diminishing pain behaviors and augmenting healthy behaviors. The operant treatment à la Fordyce (1976) is probably the prototype of a 'motoric' treatment module. Respondent treatments during which the patient learns the relaxation response, possibly supported with biofeedback can be considered the prototype of the 'physiological' treatment. An important condition is that such a treatment includes a form of exposure (such as in systematic desensitization) during which clients are gradually exposed to arousaleliciting stimuli.

Similarly, the prototype 'cognitive' treatment should have pain cognitions as their point of application. Because of the limited agreement among researchers regarding the concept of pain cognition, the group of treatments which receive the label "cognitive" consists of a host of different techniques, with all the ensuing lack of clarity. In this chapter we highlighted the role of attributions and expectations as cognitions. Expectations were hypothesized as being linked with operant conditioning and thus to be modified by consequent factors, such as actual performance. Any technique which involves performance might be expected to alter expectations. Attributions were thought of as being linked with classical conditioning, possibly taking place beyond awareness, and formed through antecedent factors. The prototype 'cognitive' treatment thus must incorporate concepts of unawareness, classical conditioning, and attribution. Some authors (Tosi \& Baisden, 1984) use therapeutic procedures that rely on hypnosis to affect non-conscious processes. In agreement with Greenberg \& Safran (1984), these authors believe that the evoking of emotional states is necessary in order to achieve changes in cognitions that take place in these states. The purpose of their evocation (e.g. through hypnosis) would not be to relieve an emotional experience, nor to promote catharsis. Rather, they would be evoked in order to elicit the attributions that are associated with the emotion. Of interest is that hypnosis is one of the oldest forms of pain management (Hilgard \& Hilgard, 1975). In the definition of hypnosis (British Medical Association report, 1955) anesthesia is explicitely noted as an effect of 
hypnosis. Finally, hypnosis also has been defined in terms of classical conditioning (Salter, 1949). Davies (1987) refers to the literature on hypnosis full of examples of redefining perceptions. These examples vary from the redefining of phobic stimull to neutral, to the redefining of pain to a healing sensation. It is remarkable that hypnosis is absent from reviews of cognitive treatments of pain. Tan (1982) excludes hypnosis, Turner \& Chapman (1982) and Turk et al. (1983) present a separate review of hypnosis and pain control, beside a review of what they call cognitive(-behavioral) treatments of pain. The latter might be hypothesized to alter expectations, while hypnosis would affect attributions.

In our view, application of the three-systems model would contribute to clarification of a discussion well described by Schmidt (1987). This author argues against the idea that a successful operant treatment implies that the chronic pain problem consequently has mainly been under operant control. Fordyce (1976) already mentioned that

"...reduction in the rates of these pain behaviors (by operant methods) does not establish conclusively that they had been occurring on an operant basis" (p. 109).

It would be interesting to investigate what effects treating one system would have on the other systems.

\subsection{Discussion}

The application of the three-systems model of emotions as a heuristic model to the study of chronic pain offers new perspectives. First, it provides a useful frame of reference for our current knowledge. Indeed, many authors have already made use of a trimodal conceptualization, although they have done so without making explicit reference to this model. Furthermore, it can help in generating new hypotheses. The concept of discordance, theoretically based on the relative interdependence of the three systems, forces us to sharpen our pain assessment methodology. A three-systems analysis may result in a pain profile, possibly leading to three types of chronic pain patients. Finally, this model offers a way of reducing the confusion that exists with regard to pain treatments and may guide future therapy evaluation research.

However, the adoption of the thee-systems model also points to gaps in our current knowledge. There are still many areas that need further investigation. Some of the most critical can be summarized as follows: 
1. A thorough analysis and consensus about the three response systems, overt-motoric behavior, pain cognitions and psychophysiology- is essential for further application of the model. The question of whether the three systems are different in chronic pain vs. acute pain, back pain vs. headache, etc. should also be addressed.

2. Reliable and valid assessment instruments for each of the three systems, and for the physiological system in particular, need to be developed.

3. Guided by the principle of parsimony, application of both the operant and classical conditioning paradigms is examined for each system, but this needs further empirical support.

4. Little is known about the etiological significance of discordance in chronic pain and about the clinical relevance of the desynchronous pain treatment course.

5. Pain treatment programs can focus on one or more of the three systems. Further research is needed to examine the relationship between a threesystem analysis and therapy outcome. This calls for the implementation of treatments in their "purest" form (operant vs. cognitive vs. respondent), as opposed to multi-modal treatment programs. 



\section{PART II:}

\section{BEHAVIORAL ASSESSMENT OF CHRONIC PAIN IN REHABILITATION}

In current behavior therapy practice, it is common use to make a functional analysis and a broad-spectrum analysis. The functional analysis (some authors use the term 'behavioral analysis') is considered the classic strategy that focuses on the complaint for which treatment is sought, and which links assessment with treatment. Simultaneously, the different response classes are identified that are believed to contribute nomothetically to a particular disorder. In accordance with the three-systems model, the functional analysis aims at mapping out the extent to which overt pain behaviors, covert pain cognitions, and physiological responses are present. Subsequently, one (or more) of these response systems would be idiographically selected for treatment in each patient. In terms of the IDH-model, the functional analysis focuses at the disability.

The broad-spectrum analysis (holistic theory) focuses on the context in which the three responses operate, and tries to identify the characteristics of the situations in which the responses occur (S), other characteristics of the patient $(\mathrm{O})$, and the short-term and long-term consequences of the responses (C). For example, the typical work environment, and possible conflicts with colleagues (S), the partner's usual response to the patients pain behavior $(C)$, and other complaints such as depression and anxiety (O) can be the subject of a broad-spectrum analysis. Examination of the patient's past history also belongs to this analysis. He/she may have had several occasions in his/ her life in which the display of suffering has resulted in a reduction of tension or stress, or in the increase of attention and reward. 
In terms of the IDH-model, the broad-spectrum analysis focuses on the Rehabilitation-Promoting, and Rehabilitation-Hindering factors, that in interaction with the impairment and disability, determine the extent to which the patient is handicapped.

This part of this dissertation only deals with the functional analysis, or chronic pain disability. For two of the three response systems of the CPS (the overt-motoric and the verbal-cognitive), valid and reliable assessment instruments are described. Chapter 3 describes the empirical examination of the dimensions and components of the "pain behavior" construct. Starting point is the broad definition of pain behavior. Chapter 4 is a logical extension of chapter 3 , and describes the development of an observational instrument for the components of pain behavior. Chapter 5 examines the validity of a Dutch version of a short American observation scale for pain behavior. One way of assessing residual health behavior in patients with CPS is through the pain-rest contingency principle. During exercise tolerance tests, patients decide themselves when to stop a certain activity. The reliabillity and validity of such a method is described in chapter 6. Finally, chapter 7 describes the development of the Pain Cognition List a questionnaire that aims to identify pain cognitions.

Chapters 3 to 5 deal with behavioral excesses of the motoric response system. In contrast, chapter 6 aims to quantify behavioral deficits, or residual motoric health behaviors. Chapter 7 focuses on both cognitive excesses (pain attributions) and deficits (residual efficacy expectations). 


\section{CHAPTER 3}

\section{Dimensions and components of observed chronic pain behavior*}

\subsection{Introduction}

Fordyce (1976), who introduced the concept of 'pain behavior', makes a distinction between a narrow and a broad definition of pain behavior. $\mathrm{He}$ states that pain behavior, narrowly defined, includes:

(1) Autonomically mediated pain indicators.

(2) Visible and audible non-verbal signals that pain is experienced

(3) Verball reports of pain.

(4) Requests for ministrations or assistance because of pain.

(5) Functional limitation or restricted movement because of pain (p. 109).

In this publication, however, Fordyce remains unclear about the broader definition of pain behaviors, stating that it is not of great importance. In a more recent publication (Loeser \& Fordyce, 1983), a broader definition of pain behavior is given, followed by a specification:

'... pain behavior [which] is the interaction between the individual and the surrounding world. Pain behavior is defined as any and all outputs of the individual that a reasonable observer would characterize as suggesting pain, such as (but not limited to) posture, facial expression, verbalizing, lying down, taking medicines, seeking medical assistance, and receiving compensation (p. 334).

The pain behavior construct according to the narrow definition conceptualized by Fordyce has been supported by empirical research. Turk et al. (1985)

\footnotetext{
* This chapter is a slightly modified version of Vlaeyen, J.W.S., van Eek, H., Groenman, N.H., \& Schuerman, J.A. (1987). Dimensions and components of observed chronic pain behavior Pain, $_{31}, 65-75$.
} 
employed multidimensional scaling and hierarchical clustering statistical techniques to identify the latent structure of pain behavior. They found that pain behavior can be characterized by 2 dimensions, namely 'audiblevisible" and "affective-behavioral'. Four clusters of pain behavior were identified and labeled: distorted ambulation or posture, negative affect, facial/audible expressions of distress, and avoidance of activity (Table 3.I).

Although Turk's study presents an interesting design, the question is whether the results have not been influenced by circularity. The original list of behaviors was selected from the literature, including Fordyce (1976) and Sanders (1979) and was thus influenced by Fordyce's ideas. After a first selection, the behaviors were sorted by pain specialists. They were members of the International Association for the Study of Pain (IASP) and were probably familiar with this conceptualization. Therefore, it is not surprising that the latent structure turns out to be generally congruent with Fordyce's original conceptualization.

Another empirical study in which the components of pain behavior are examined was done by Philips and Jahanshahi (1985). These authors presented a checklist consisting of 49 items to 267 headache patients. A factor analysis resulted in 13 factors (Table $3 . \mathrm{I}$ ). Based on these results the authors constructed an assessment instrument, in the form of a pain behavior checklist, giving information about the presence of the components of headache behavior. In this study no raters or observers were employed. The results are based on self-reported material and therefore subject to distorting factors, as discussed earlier. The possibility of transforming the checklist into an observation scale was not considered by the authors. Philips and Jahanshahi, however, conclude that the checklist has to be completed with other, more objective material.

Our study is designed to be a replication of the study of Turk et al. (1985), but with changed conditions:

1. In contrast to Turk, our starting point is the broader definition of pain behavior, namely, the interaction between the pain patient and his direct environment.

2. The original behavior items are not selected from the existing literature, but collected by nurses. These nurses are, at least in the clinical setting, members of the direct environment and able to observe the patient carefully.

3. In order to a void the problem of circularity we chose not to work with IASP members as raters, but with a more neutral group of behavioral scientists. 
Table 3.I. Empirical examinations of the components of pain behavior:

$\begin{array}{lll}\text { Authors } & \text { Turk et al. (1985) } & \text { Philips and Jahanshahi (1985) } \\ \text { Source } & \text { Literature } & \text { Self-report by headache patients } \\ \text { Procedure } & \text { Cluster analysis } & \text { Factor analysis }\end{array}$

Components 1. Distorted ambulation of posture 1. Social avoidance

2. Negative affect

3. Facial/audible expression of

2. Avoidance of housework activities distress

4. Avoidance of activity

3. Daily mobility avoidance

4. Activities avoidance

5. Daily exercise avoidance

6. Stimulation avoidance

7. Non-verbal complaint

8. Verbal complaint

9. Self-help strategies $A$

10. Self-help strategies B

11. Medication

12. Crying

13. Distraction

\subsection{Subjects}

Information gathered by 12 nurses from the Department of Rheumatology of the Lucas Foundation for Rehabilitation who participated in an earlier study (Vlaeyen et al., 1985) was used in the first phase of the study. Ninetysix psychologists and members of the Flemish and Dutch Associations for Behavior Therapy served as subjects for the second phase of the study.

\subsection{Procedure}

During the first phase of the study data from the earlier study were used. In this study 12 nurses were asked to carefully observe 21 male and 26 female inpatients, admitted to the Department of Rheumatology of the Lucas Foundation for Rehabilitation, for chronic back pain problems. Patients' mean ages were 39.1 and 52.0 years respectively. Nineteen patients $(40.4 \%)$ were diagnosed as having chronic low back pain, $25.5 \%$ suffered from spondylitis ankylopoetica, $25.5 \%$ had rheumatoid arthritis and $8.5 \%$ received 
the diagnosis fibromyalgia. The systematic recording of these observations resulted in a list of 232 behavior items. For the present study only items indicating behavior which hampers the development of well-behavior were selected. Observations with great similarity were reformulated into 1 item. The final list consisted of 78 behavior items.

In the second phase, the 78 items, printed on small filings cards, were presented to the 96 psychologists with the following instruction:

You receive 78 examples of behaviors of chronic pain patients reported by nurses in a rehabilitation clinic. Please cluster these behaviors into categories based on whatever criteria that you believe are relevant. Do this by sorting the cards on which the behavior descriptions are printed. Behaviors that you feel belong together should be assigned to the same group. You can make as many groups as you feel are necessary'.

Every psychologist produced a certain number of groups with a certain number of items. For all psychologists, the frequency of occurrence of 2 items in the same group was calculated. This information allows the construction of a $78 \times 78$ frequency matrix. Each cell represents the frequency with which 2 items coincide in the same group. These frequencies varied between 0 (pairs of items never placed together) and 96 (pairs of items always placed in the same group).

\subsection{Results}

The statistical techniques used were the same as in the Turk study, namely multidimensional scaling (MDS) and hierarchical cluster analysis (HCA). MDS is a procedure aimed at the discovery of the latent structure of a data pool, when the degree of similarity between the items is known. MDS differentiates the items in various dimensions. The result is a multidimensional representation of the items in the form of a geometrical configuration of points. In this configuration, items with greater similarity are put closer to each other. Larger distances correspond to less similarity (Kruskal \& Wish, 1978). The dimensions on the basis of which the 96 psychologists sorted the 78 items were analyzed by means of the statistical MDS package Minissa, version 3.10 (Davies \& Coxon, 1983). Solutions in 1-8 dimensions were obtained. On the basis of the respective stress values (Fig. 3.1) we concluded that 3 dimensions were most appropriate for the interpretation of the results. 
The assignment of labels to the dimensions was based on the examination of the behavior items falling at the extreme points of each dimension.

For dimension 1, behaviors lying at one pole include 'behaves passively' and 'withdraws from other people', while at the other pole behaviors such as 'cries' and 'requests pain medication' are found. We felt that we could assign the label 'withdrawal-approach' to this dimension.

Behaviors lying at one extreme of dimension 2 include 'is obtrusive', "is restless and nervous' and at the other extreme we find 'walks guardedly and carefully', 'takes a nap after lunch'. This dimension is labeled 'high arousal-low arousal'. When comparing extreme items of this dimension with those of Turk's dimension 'affective-behavioral', a high degree of similarity can be seen. However, we chose to label this dimension differently.

Finally, examination of the third dimension reveals that 'changes position frequently while sitting' and 'walks with abnormal gait' are found on one extreme and "asks for lighter therapy program", 'mentions having more pain after therapy' on the other. This dimension shows much similarity with the dimension 'visible-audible' in the study of Turk and his colleagues. Therefore, the same label is given to this dimension.

The next step consisted of looking at the different behavior components. The original similarity matrix was transformed into a distance matrix and subsequently submitted to HCA. HCA is aimed at grouping items with a relatively high degree of similarity (Everrit, 1974), in contrast to MDS, which helps to differentiate items.

HCA resulted in 9 different clusters of behaviors. Fig. 3.2 shows these clusters embedded in the geographical MDS representation. Based on the inspection of the items of each cluster, listed in Table 3.II, the clusters were

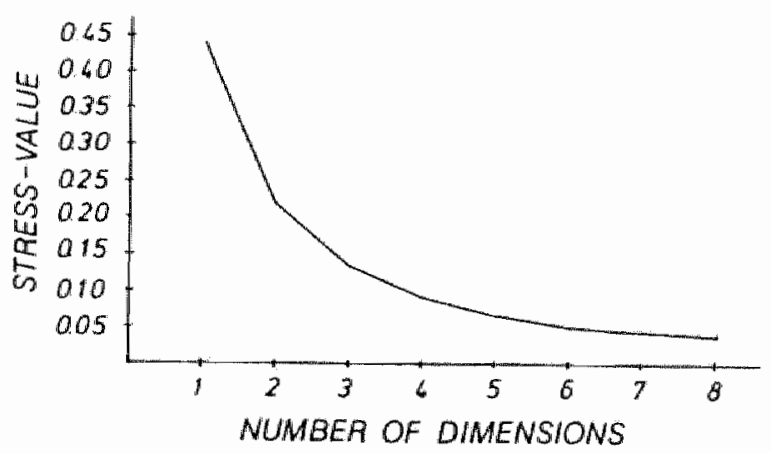

Figure 3.1. MDS stress values at each of the 8 dimensions. 
Table 3.II. Hievarchical cluster analysis of pain behaviors. Items are translated from the Dutch. Letters in parentheses correspond to letters in figure

3.2 .

Cluster

1: Anxiety

2: Altention seeking

3: Verbal pain complaints

4: Medication use
Item

1. Tense (A)

15. Restless and nervous

27. Is in panic (B)

41. Nervous when having difficulties doing something

19. Mentions feeling anxious and tense

73. Blushes and sweats

16. Interrupts and disturbs others' activities

49. Talks continuously

69. Is obtrusive (C)

60. Claims the attention of nurses and other patients (D)

5. Mentions having more pain after therapy

26. Gives lively descriptions of the pain (E)

36. Says that she/he cannot stand the pain anymore

65. Complains about pain attacks

71. Complains abou" pain

76. Says the pain has not diminished

18. Rubs painful parts of the body (F)

75. Cries

24. Groans, moans of sighs (G)

25. Takes pain medication $(\mathrm{H})$

38. Takes more pain medication than prescribed (I)

55. Requests pain medication (J)

31. Takes sleep medication $(\mathrm{K})$

66. Takes antidepressants (L)

5: General verbal complaints 6. Complains about stiffness after therapy

48. Complains about stiffness after sitting (M)

61. Complains about morning stiffness

8. Asks for further specialized medical treatment

42. Asks for a consultation with the psychologist

39. Asks for help

12. Asks for a lighter therapy program

22. Says that the program is too hard (N)

35. Is querulous

32. Mentions being bored (O)

6: Distorted mobility and posture
9. Needs help rising after sitting for a while

10. Needs help leaving the bed 
20. Wears a brace $(P)$

37. Walks with crutches from room to the common room

45. Uses a wheelchair to go to therapy

52. Has problems with activities of daily living

17. Moves rigidly and stiffly, with stretched limbs

43. Stands in an unusual posture

33. Walks with abnormal gait

56. Has bad mobility

64. Walks guardedly and carefully

63. Walks with dragging steps (Q)

21. Changes from one buttock to the other while sitting

78. Changes position frequently while sitting

53. Grimaces $(R)$

58. Moves rigidly and shyly (S)

7: Fatigue

2. Falls asleep during the day

34. Lies in bed during the weekend

28. Lies down between therapy sessions

14. Takes a nap after lunch ( $T$ )

30. Sits between therapy sessions

70. Rests on the way to therapy sessions

72. Asks for a resting pause (U)

74. Sleeps between therapy sessions

8: Insomnia

40. Falls asleep early in the morning

57. Has difficulties falling asleep

59. Wakes up at night (V)

47. Awakes early in the morning $(W)$

9. Depression

3. Behaves passively

50. Does not take any initiative

46. Does only what has been asked

51. Misses therapy sessions if not reminded

68. Undertakes nothing outside therapy time

4. Is quiet and withdrawn

29. Isolates her/himself

44. Is listless

67. Is blue, down $(X)$

54. Worries

7. Talks about suicide

62. Talks in a pessimistic way

23. Says things are not working out well $(\gamma)$

77. Mentions not being interested when encouraged to undertake something

11. Appears drowsy and sleepy

13. Says that she/he is tired $(Z)$ 
labeled as: (1) anxiety; (2) attention seeking; (3) verbal pain complaints; (4) medication use; (5) general verbal complaints; (6) distorted mobility and posture; (7) fatigue; (8) insomnia; and (9) depression.

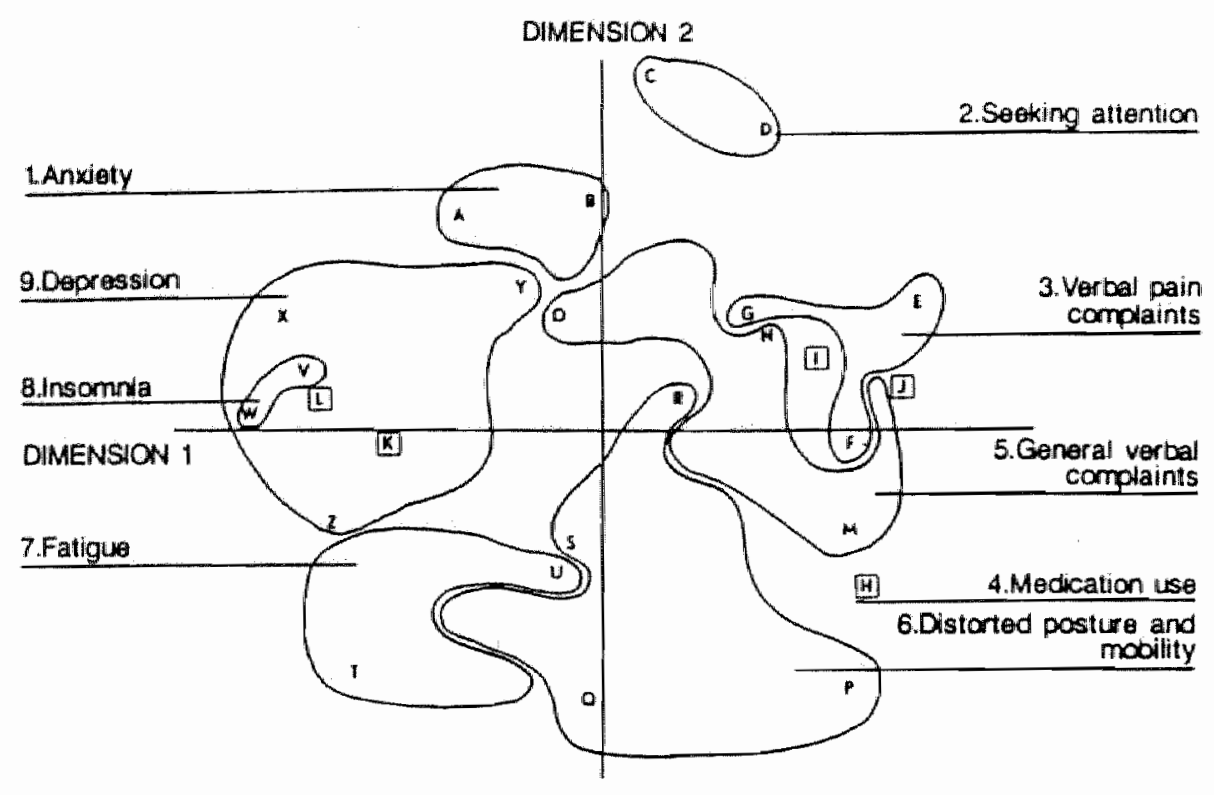

Figure 3.2. Graphical MDS 2-dimensional representation with clusters embedded.

\subsection{Discussion}

Chronic pain behavior, considered as the overt-motoric factor of the chronic pain syndrome and defined as the interaction between the chronic pain patient and his/her direct environment, seems to be composed of 9 components and to be characterized by 3 dimensions.

The dimensions and components found in our study appear to differ slightly from those found by Turk and his colleagues (1985). We opted for a broader definition of pain behavior than Turk, and found one more dimension and about twice as many components. The results of our study 
suggest that chronic pain patients present a behavior pattern which is more extensive than the typical pain behaviors found in the literature. Anxiety, depression and insomnia are not only reported in relation to chronic pain (Savitz, 1985; Sternbach, 1974; Crook \& Tunks, 1985), these complaints have been empirically confirmed in our study.

The differences with the study of Philips and Jahanshahi (1986) are more significant. Only 4 of their components (medication use, verbal complaints, non-verbal complaints and avoidance of mobility) are confirmed in our study. One of the reasons might be the different conditions of the experiment. Philips and Jahanshahi used self-reported behavior items including items indicating well-behavior. Of interest is that their results, compared to ours, suggest that headache patients display another pain behavior pattern than our chronic pain patient population.

The results not only give insight into the latent structure of the 'broader' pain behavior construct as introduced by Fordyce (1976) and Loeser \& Fordyce (1983), they also provide a basis for the construction on an observation scale for the instrumental factor of chronic pain, or overt pain behavior. The validity of the clusters found could be assessed by means of a checklist in which the 78 items are provided with a rating scale. This checklist could then be used by nurses or trained observers. Moreover, such a checklist would provide the possibility of comparing pain patients with different diagnoses, and thus contribute to the assessment of chronic pain in general.

For each patient an observation profile could be formed, which could provide information about at least 2 aspects of the observed chronic pain behavior. First, the position of the patients' pain behavior in relation to the 3 dimensions can be calculated, as MDS indicates a loading for each item with respect to each of the 3 dimensions. Second, as every item belongs to 1 of the 9 clusters, a score for each cluster can also be calculated, based on the ratings given to the items of the respective clusters.

Assessment should be related to treatment. Although answers are premature at this moment, the question arises whether on the basis of the profile proposed above, conclusions could be drawn about the therapeutic intervention to be chosen. It is not impossible that patients scoring higher on the dimension "high arousal" can benefit more from respondent techniques such as relaxation. High scorers in the direction of "approach' are probably sensitive to an operant approach, aimed at abolishing excessive behavior and shaping more adequate well-behavior. Finally, pain behavior merely characterized by 'withdrawal' might be more controlled by cognitive factors or the pain experience. In this case cognitive-behavioral interventions might be more appropriate. 
More clinical evaluation research, including research towards the predictive value of specific assessment procedures, is essential in order to test these tentatively formulated hypotheses. 


\section{Assessment of the components of observed chronic pain behavior: the Checklist for Interpersonal Pain Behavior (CHIP)*}

\subsection{Introduction}

Under the influence of Fordyce's theory of operant conditioning of pain behaviors (Fordyce, 1976), a great deal of clinical research has dealt with the modification of overt expressions of pain and suffering (Linton, 1986). The overall result is that pain behaviors can be extinguished successfully and that healthy behaviors can be shaped through operant conditioning programs. However, in order to evaluate the effectiveness of these programs, reliable and valid instruments are needed to measure pain behaviors. One recurrent problem that characterizes attempts to assess pain behaviors involves the characteristics of the 'pain behavior' construct. There is little agreement about the range of behaviors that should be assessed (Turk et al., 1985). This problem has stimulated researchers to empirically examine the dimensions and components of the pain behavior construct (Philips \& Jahanshahi, 1986; Turk et al., 1986; Vlaeyen et al., 1987). These studies provide an empirically derived and methodologically sound basis for the construction of a pain behavior measure.

In the previous chapter, we replicated the study of Turk et al. (1986) and found that pain behavior -in the broad sense of the term-can be characterized along three dimensions: 'withdrawal-approach', 'high arousal-Low arousal', and 'visible-audible". In addition, nine components were identified:

* This chapter is a slightly modified version of Vlaeyen, J.W.S., Pernot, D.F.M., Kole-Snijders, A.M.J., Schuerman, J.A., van Eek, H., \& Groenman, N.H. (1990). Assessment of the components of observed chronic pain behavior: The Checklist for Interpersonal Pain Behavior (CHUP). Pain, 43, 337-347. 
1. anxiety

2. attention seeking

3. verbal pain complaints

4. medication use

5. general verbal complaints

6. distorted posture and mobility

7. fatigue

8. insomnia

9. depressive mood

Pain behaviors were generated by nurses who carefully observed chronic back pain patients. The 78 items found were subsequently sorted by 96 psychologists. It was suggested that the same items could provide a useful basis for the development of an observational measurement technique for chronic pain.

The present study is designed to be an extension of the above-mentioned study, and is an attempt to transform the original pool of 78 items into a reliable and valid observation scale for pain behavior. A device which directly measures pain behavior in the broad sense, as identified in the above mentioned study would represent a useful addition to the diagnostic protocol of pain management centers. For the purpose of clarity, and in order to differentiate between the narrow and broad definition of 'pain behavior', the term 'interpersonal pain behavior' is introduced, referring to the broad definition.

Six studies will be presented. The first study examines the factor structure of this new observational instrument. Subsequently, two studies are described which focus on the reliability of the CHIP. Finally, three studies examine the validity of the instrument. All of the studies were carried out with patients admitted to an inpatient rehabilitation program at the Department of Rheumatology of the Lucas Foundation for Rehabilitation in Hoensbroek, The Netherlands.

\subsection{Study I: Factor structure*}

The 78 items derived from the previous study were transformed into a checklist in which they are followed by a 5 -point Likert scale. By choosing one of the five alternatives ("never observed', 'seldomly observed", 'occasionally observed', 'frequently observed' and 'almost always observed')

"Copies and norms of the CHIP can be obtained from the author. 
the frequency of occurrence of each item could be rated. This new observational method was named ' Checklist for Interpersonal Pain behavior (CHIP) The purpose of study I was to analyze the factor structure of the CHIP and to evaluate the characteristics of the factors found.

\subsubsection{Subjects}

Eighty two female and 70 male chronic pain patients consecutively admitted to an inpatient rehabilitation program were carefully observed during their first two weeks of admission by one of 12 nurses. The patients' mean ages were $47.4(\mathrm{SD}=14.9)$ and $39.6(\mathrm{SD}=13.6)$ years, respectively. The sample was diagnostically heterogeneous. Seventy-two patients $(47.4 \%)$ were diagnosed as having chronic back pain (CBP), $58(38.2 \%)$ suffered from rheumatoid arthritis, $12(7.8 \%)$ suffered from spondylitis ankylopoetica, and $10(6.6 \%)$ were diagnosed as having fibromyalgia. One hundred and twenty-four patients $(78 \%)$ were admitted for chronic back pain problems.

\subsubsection{Procedure}

The CHIP was used by one of a group of 12 nurses to quantify observed pain behaviors. The observation took place during the first week of admission, after which the checklist was filled in for each patient. The 152 checklists were subsequently subjected to frequency counts and the frequency distribution was analyzed. Only those items with a frequency distribution approximating the normal distribution were accepted. Items with a skewness $<-1$ and $>1$, and items which were not observed in more than $10 \%$ of the cases were rejected. A principal component factor analysis with Varimax rotation was applied to the remaining items. Items with a communality < 0.30 and items with a factor loading $<0.40$ on all of the factors were also rejected.

Finally, an analysis of variance (ANOVA) was carried out in order to examine the relationship between factor scores and individual differences including sex, age (under versus over the median $=43.5$ years), and diagnosis (CBP versus other diagnoses group).

\subsubsection{Results and discussion}

Analysis of the frequency distribution of the 78 items revealed that only 34 items were quasi-normally distributed. Six items were not observed in more than $10 \%$ of the cases. A principal component factor analysis was carried out on the remaining 28 items. Using the criterion of factors with eigenvalues $>1$ to determine the number of factors to be extracted, 6 factors were obtained which cumulatively accounted for $67.1 \%$ of the total variance. To maximize the variance of the squared factor loadings, the original 
6-factor solution was subsequently subjected to an orthogonal Varimax rotation. Item loadings $\geq 0.40$ were considered significant. Using this criterion, item 67 showed high loadings on both factor 4 and factor 5 . Therefore, Pearson correlation coefficients between item 67 and factors 4 and 5 were calculated $(0.72$ and 0.81 , respectively, both $\mathrm{p}<0.001)$. As the correlation with factor 5 was greater, item 67 was assigned to this factor. Factors were labelled following the common procedure of giving the greatest consideration to items with high loadings on each factor. The following labels were assigned to the factors: 'Distorted Mobility' (CHIP-DM), 'Verbal Complaints' (CHIP-VC), 'Non-verbal Complaints' (CHIP-NC), 'Nervousness' (CHIPN), 'Depression' (CHIP-D), and 'Day-sleeping' (CHIP-DS). Table 4.I shows the items with their respective factor loadings. Taking into account the reduced item pool -only $36 \%$ of the original items could be included in the factor-analysis- the factor structure does correspond fairly well with the clusters found in the earlier study.

Characteristics of the CHIP factors including factor label, number of items per factor, score range, skewness, factor means and standard deviations, and Cronbach's alpha are presented in Table 4.II. All CHIP factors appeared to be quasi normally distributed (skewness $<1$ ). For factors 1 to 5 Cronbach's alpha, the widely accepted measure for internal consistency of a test, ranged from 0.83 to 0.89 , suggesting excellent internal consistency. Because of its poor internal consistency and its limited number of items, CHIP factor 6 is excluded from further analyses.

The ANOVA, testing the relationship between factor scores and individual differences, showed significant differences between the male and the female patients for all factors $(p<0.01)$. This finding suggests that female pain patients showed more interpersonal pain behavior than the male pain patients. For factors 3 and 5 significant differences emerged between the two age groups $(F=4.86, p=0.03$ and $F=4.15, p=0.04$ respectively), suggesting that older pain patients displayed more non-verbal complaints and showed more depressed behavior than younger pain patients. For factors 2 and 3, ANOVA revealed significant differences between the diagnosis groups $(F=4.01, p=0.048$ and $F=5.84, p=0.02$ respectively), suggesting that $C B P$ patients displayed more verbal and non-verbal pain behavior than the other diagnoses group. Interestingly, for factors 1, 4, and 5, significant 2way interactions emerged between sex and diagnosis.

A post hoc t-test revealed that within the female group, the CBP patients scored significantly higher than the other diagnoses group on these factors $(t=2.54, p<0.01 ; t=1.75, p<0.05$; and $t=3.72, p<0.001$ respectively). This was not the case for the male patient group. For factor 1, male CBP 
Table 4.I. Varimax rotated factor matrix and reliability coefficients for the individual items (translated from the Dutch). Numbers refer to the original behavior list. A: intra-rater reliability. B: inter-rater reliability (Pearson product-moment correlation coefficients for both $A$ en $B$.)

\begin{tabular}{|c|c|c|c|c|c|c|c|c|}
\hline \multirow{2}{*}{ Item } & \multicolumn{6}{|c|}{ CHIP Factor } & \multirow{2}{*}{ A } & \multirow{2}{*}{ B } \\
\hline & 1 & 2 & 3 & 4 & 5 & 6 & & \\
\hline 33. Walks with abnormal gait & \multicolumn{5}{|l|}{0.83} & & \multicolumn{2}{|c|}{$.60^{* *} .85^{* *}$} \\
\hline 43. Stands in unusual posture & \multirow{2}{*}{\multicolumn{5}{|c|}{0.80}} & & \multicolumn{2}{|c|}{$.57^{* *} .53^{* *}$} \\
\hline 17. Moves rigidly and stiffly, & & \multicolumn{4}{|c|}{0.75} & & \multicolumn{2}{|c|}{$.62^{* *} .50^{* *}$} \\
\hline 64. Walks guardedly and carefully & \multicolumn{5}{|c|}{0.61} & & \multicolumn{2}{|c|}{$.70^{* *} .65^{* *}$} \\
\hline 56. Has bad mobility & \multicolumn{5}{|c|}{0.60} & & \multicolumn{2}{|c|}{$.49^{* *} .51^{* 4}$} \\
\hline 63. Drags feet when walking & \multicolumn{5}{|l|}{0.44} & & \multicolumn{2}{|c|}{$.30 .43^{*}$} \\
\hline \multicolumn{2}{|l|}{ 71. Complains about pain } & \multicolumn{4}{|l|}{0.70} & & \multicolumn{2}{|c|}{$.57^{* *} .73^{* *}$} \\
\hline \multicolumn{6}{|c|}{ 26. Gives lively descriptions of the pain 0.63} & & \multicolumn{2}{|l|}{$.55^{* *}$} \\
\hline \multicolumn{6}{|c|}{ 76. Says that the pain has not diminished 0.59} & & \multicolumn{2}{|c|}{$.72^{* *} .74^{* * 4}$} \\
\hline \multicolumn{9}{|c|}{ 5. Mentions having more pain after } \\
\hline therapy & & \multicolumn{4}{|l|}{0.55} & & \multicolumn{2}{|c|}{$.67^{3+4} .22$} \\
\hline \multicolumn{2}{|l|}{ 13. Says that she/he is tired } & \multicolumn{2}{|l|}{0.47} & & & & \multicolumn{2}{|c|}{$.48^{* *} .48^{* *}$} \\
\hline \multicolumn{9}{|c|}{$\begin{array}{l}\text { 21. Changes from one buttock to the other } \\
\text { while sitting }\end{array}$} \\
\hline while sitting & & & 0.79 & & & & .12 & $.43^{*}$ \\
\hline 78. Changes position frequently & & & & & & & & \\
\hline while sitting & & & 0.77 & & & & $.42^{*}$ & $.58 *$ \\
\hline 18. Rubs painful parts of the body & & & 0.56 & & & & $.47^{* *}$ & $.60^{* 4}$ \\
\hline 24. Groans, moans or sighs & & & 0.51 & & & & $.40^{*}$ & $.59 * *$ \\
\hline 15. Restless and nervous & & & & 0.75 & & & $.48^{* *}$ & $.80^{* * *}$ \\
\hline 1. Tense & & & & 0.70 & & & $.57^{* *}$ & $.57 * *$ \\
\hline 54. Worries & & & & 0.54 & & & $.36^{*}=$ & .08 \\
\hline 4. Is quiet and withdrawn & & & & & 0.84 & & $.51^{* *}$ & $.60^{* 4}$ \\
\hline 29. Isolates her/himself & & & & & 0.73 & & $.72^{* 4}$ & $.63^{4 * 4}$ \\
\hline 11. Appears drowsy and sleepy & & & & & 0.42 & & $.49^{* *}$ & $.50^{4 *}$ \\
\hline 67. Appears blue, down & & & & 0.49 & 0.41 & & .33 & $.38^{*}$ \\
\hline 2. Falls asleep during the day & & & & & & 0.89 & & \\
\hline 14. Takes a nap after lunch & & & & & & 0.61 & & \\
\hline
\end{tabular}

${ }^{*} \mathrm{p}<.01$, ** $\mathrm{p}<.001$ 


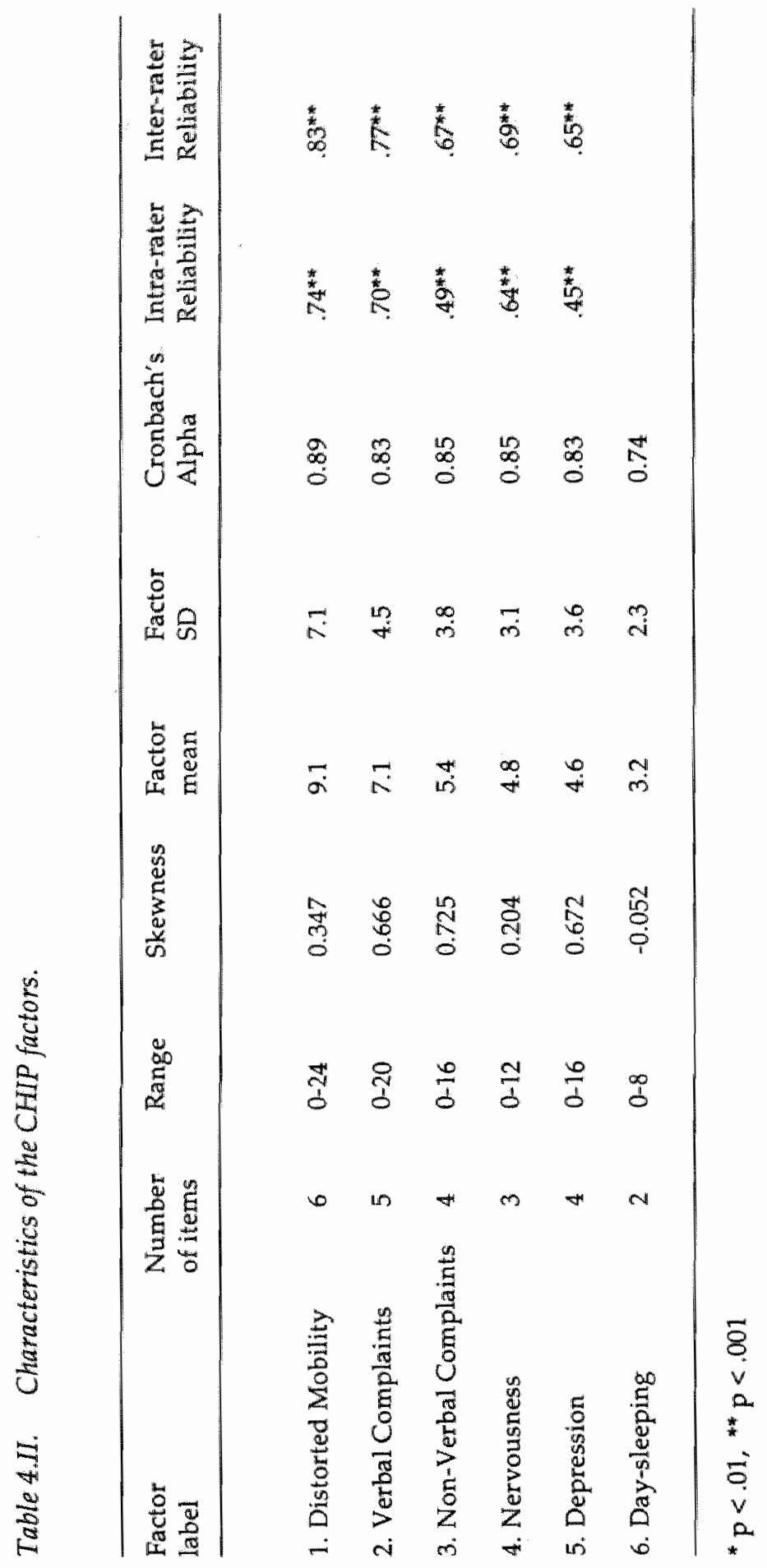


patients even scored significantly lower than the male other diagnoses group ( $t=-2.23, \mathrm{p}<0.025)$.

These results suggest that, in general, female pain patients display more interpersonal pain behavior than male pain patients, and that this is more pronounced for female CBP patients who appear to display more distorted mobility, nervousness and depressive behaviors than female patients with other diagnoses. Moreover, CBP patients show more verbal and non-verbal pain behavior than patients with other pain diagnoses. This means that if norms are to be developed, different norm groups including both sexes, age groups and diagnosis groups will have to be taken into consideration.

In order to utilize the 22-item CHIP as an observational measurement instrument, the following studies examined both reliability and validity of the CHIP factors. The patients serving as subjects of these studies were classified by their physicians as either having minimal organic findings or displaying pain complaints disproportionate to the demonstrable organic basis of their pain.

\subsection{STUDY II: Intra-rater reliability}

Reliability analysis of the CHIP means assessing internal consistency, intrarater reliability, and inter-rater reliability for all internally consistent CHIP factors. Study II assessed the intra-rater reliability of the individual CHIP items and CHIP factors by examining correlations between two successive observations.

\subsubsection{Subjects}

Thirteen male and 33 female chronic low back pain patients, admitted for an inpatient rehabilitation program, were observed at two different points in time. Their mean ages were 40 years (range, 28 to 52 years) and 44 years (range, 24 to 60 years) respectively. The mean duration of their pain complaints was reported to be 11.9 years, ranging between 1 and 37 years.

\subsubsection{Procedure}

The observation took place during two pain screening days being held before admission to the rehabilitation center. The time interval between the two observations was 2 weeks. During the screening days, a trained research assistant scored the CHIP while exercise tolerance levels were assessed by means of a standardized instruction which was based on the pain-rest contingency principle: "Carry on until pain or physical discomfort makes you stop the activity.". Both dynamic activities (walking, riding on 
a bicycle ergometer, arm and leg rowing, and shifting objects from one place to another) and static activities (sitting and standing) were requested from the patients. Pearson product-moment correlation coefficients were calculated between the two successive administrations for the individual CHIP items and for the 5 CHIP factor scores.

As the subjects in this study also participated in study V and study VI, the level of significance was conservatively set at .01.

\subsubsection{Results and discussion}

As can be seen in Table $4 . I$ (column A), only three items (21, 63 and 67) appear to be insufficiently reliable. Table 4 .II displays the reliability coefficients for the 5 CHIP factors, which are all significant. These results suggest that the CHIP is stable across time, at least when being used by trained observers who are able to carefully observe pain patients who are engaged in physical activities.

\subsection{Study III: Inter-rater reliability.}

Study III evaluated the inter-rater reliability of the CHIP, by examining the correlations between scores of the individual CHIP items and CHIP factors obtained by two independent observers.

\subsubsection{Subjects}

The sample of this study consisted of 37 female and 10 male chronic low back pain patients with mean ages 44.8 years (range, 24 to 64 years) and 35.3 years (range, 27 to 52 years) respectively. The mean duration of their pain complaints was reported to be 12.2 years ranging between 6 months and 31 years.

\subsubsection{Procedure}

The procedure was similar to the one in study II. The inter-rater reliability analysis was carried out with two trained research assistants who independently observed the patients during a measurement day on which exercise tolerance levels were assessed as described in the previous study. The observation took place two weeks after admission.

\subsubsection{Results and discussion}

Pearson product-moment correlation coefficients between the scores for the individual CHIP items revealed significant inter-rater reliability for all items except items 5 and 54 (Table 4.I, column B). Correlations for items 63, 
21 and 67 are significant but low. For the 5 CHIP factor scores, significant correlations are found (Table 4.II). These results suggest that the inter-rater reliability of the CHIP is acceptable. However, because of their weak intraand interrater reliability, removal of items $21,54,63$, and 67 from the CHIP might be considered in future research.*

\subsection{Study IV: Validity of CHIP-DM and CHIP-NC}

The heterogeneous character of the CHIP factors makes it difficult to examine validity in a simple way. In fact, validity for each individual factor should be assessed. Nevertheless, one form of validity, content validity, is assumed to be sufficient for all CHIP factors. Content validity is determined by the adequacy with which an instrument samples the behavioral domain of interest (Cronbach, 1971). As the CHIP is directly derived from an empirical study that examined the dimensions and components of pain behavior, it may be concluded that content validity has already been proven.

Study IV assessed the criterion-related validity of CHIP factors DM and NC. Criterion-related validity, also referred to as concurrent validity, refers to the usefulness or accuracy of scores in predicting some performance criterion or in substituting for some other, more costly, measurement procedure (Barrios \& Hartman, 1986; Mischel, 1986). In study IV, the extent to which the CHIP scores correspond with the number of pain behaviors counted on videotapes is examined. Correlations between the CHIP scores and another existing observational instrument are also analyzed.

\subsubsection{Subjects}

In this study, the subject sample consisted of 15 female and 4 male chronic low back pain patients with mean ages 43.7 (range, 24 to 55 years) and 38.7 years (range, 29 to 52 years) respectively. Thirty video recordings of these patients were made while their functional skills were being assessed using the pain-rest contingency principle as described in study II.

\subsubsection{Procedure}

The Audiovisual Taxonomy for Assessing Pain Behavior (ATAPB), developed by Keefe et al. (1987), and a Dutch version of Richards et al.'s (1982) UAB Pain Behavior Scale (PGS; Vlaeyen et al., 1990), were chosen as con-

\footnotetext{
* Removal of items 21,54,63 and 67 affects internal consistency of CHIP factors 1, 3, 4 and 5. Cronbach's Alpha changes from $.89, .85, .85$, and .83 to $.88, .77, .85$, and 79 respectively.
} 
current measures for CHIP factors "distorted mobility' and "non-werbal pain complaints'.

The ATAPB allows five behavior categories to be observed, namely sighing, bracing, guarding, rubbing, grimacing, and a total score. Psychometric analysis of the ATAPB (Kleinke \& Spangler, 1988) showed that the total score has an acceptable reliability, and that the individual pain behaviors do not occur at sufficient frequencies to be considered alone. In this study, it was decided to compute both the individual behavior scores and the total scores.

The PGS originally consists of 10 target pain behaviors assumed by Richards et al. (1982) to be reliably observed in the chronic pain population. In agreement with the study of Feuerstein et al. (1985) we found that only 8 of the 10 original behaviors can be reliably observed when having the disposal over limited observation time (see chapter 5).

Both CHIP and PGS ratings were obtained on the same days that video recordings took place. The audiovisual taping was done individually and patients were aware of the videotaping. According to Keefe et al.'s study, chronic pain patients were videotaped while involved in physical activities as described in study III. Each videotaped session was divided into segments of 30 seconds. To ensure consistency in the intervals, a recorded audio signal was dubbed onto each videotape. Depending on the length of the video recording, a different number of segments was obtained. After each segment, 10 seconds were used to rate the ATAPB. Scores were derived for each category by adding the total number of occurrences of each coding category over the observation session. In order to obtain comparable values, this score was divided by the number of segments and then multiplied by 100. A total pain behavior score consisted of the sum of each category score. For the total pain behavior score inter-observer agreement between two trained observers who both scored 15 video recordings was 0.94 ( $\mathrm{p}<$ 0.001 ). One of them completed the 30 recordings, and the data derived from her observations were included in further analyses. Because of the relatively small sample size, Kendall correlation coefficient Tau is chosen in this study.

\subsubsection{Results and discussion}

Correlations between scores for CHIP factors "distorted mobility' and 'nonverbal complaints', on the one hand, and ATAPB scores and PGS scores on the other, were calculated and are summarized in Table 4.III. It should be noted that similar to the study of Kleinke and Spangler (1988), 'sighing' and 'rubbing' occurred at very low frequencies. The two CHIP factors 'distorted mobility' and 'non-verbal complaints' correlate significantly with 
both the ATAPB total scores and the PGS scores, indicating a good criterion-related validity. Of interest is that the PGS displays a pattern of sigrificant correlations with the ATAPB scores similar to the CHIP factors DM and NC.

Table 4.III. Criterion-related validity for CHIP factors DM and NC: Kendall correlation coefficient Tau with ATAPB: Audiovisual Taxonomy for Assessing Pain Behavior and PBS: Pain Behavior Scale.

\begin{tabular}{llllllll}
\hline CHIP-factors & \multicolumn{5}{c}{ ATAPB categories } & PGS \\
\hline & sighing & bracing & guarding & rubbing & grimacing & total \\
CHIP-DM & 0.09 & $0.65^{* * *}$ & $0.50^{* * *}$ & 0.21 & 0.11 & $0.43^{* *}$ & $0.52^{* * *}$ \\
CHIP-NC & 0.27 & $0.79^{* * *}$ & $0.75^{* * *}$ & 0.25 & $0.53^{* *}$ & $0.81^{* * *}$ & $0.81^{* * *}$ \\
PGS & 0.22 & $0.58^{* * *}$ & $0.56^{* * *}$ & $0.28^{*}$ & $0.41^{* *}$ & $0.62^{* * *}$ & \\
\hline
\end{tabular}

${ }^{*} \mathrm{p}<0.05,{ }^{* *} \mathrm{p}<0.01,{ }^{* * *} \mathrm{p}<0.001$ (one-tailed)

\subsection{Study V: Validity of CHIP-DM, CHIP-VC and CHIP-NC}

This study assessed the construct validity of $\mathrm{CHIP}$ factors DM, VC, and NC by examining the relationship between the CHIP scores and measures of health behavior. Pain behaviors are conceptualized to be incompatible with health behaviors such as physical performance (Fordyce et al., 1982). Therefore, it was hypothesized that negative correlations are to be found among CHIP factor-scores and measures of physical performance.

\subsubsection{Subjects}

In study $V$, the subjects were the same as those involved in study II.

\subsubsection{Procedure}

The procedure is similar to the one described in study II, but on the second observation day, exercise tolerance levels were recorded. The patients were asked to walk, to ride a stationary bicycle, and to perform desk work in both the standing and sitting position. To assess exercise tolerance, patients were asked to perform the target activity "...until pain or physical discomfort causes you to wish to stop.". The distance walked, and the time 
spent riding the bicycle, standing and sitting were recorded. Pearson product-moment correlations were calculated among these measures of health behavior and scores on CHIP factors DM, VC, and NC.

\subsubsection{Results and discussion}

As displayed in table 4.IV, an interesting differential pattern of significant correlations emerged among CHIP factor scores and exercise tolerance measures. All of the correlations are significant, except for CHIP factor VC. Correlations with walking and bicycle riding are non- existent. This finding suggests that verbal complaints may also occur with pain patients who walk long distances and spend a longer time on the bicycle. In accordance with the hypothesis, and with the item content of CHIP factor DM, correlations with this factor are more pronounced for the exercises involving mobility (walking and bicycle riding) as compared to the other, more stationary exercises $(-.51,-.60$ versus $-.37,-.31)$. A similar pattern is seen for CHIP factor NC. Correlations are greater for activities involving sitting (bicycle riding and sitting), as compared to the exercises involving walking and standing $(-.38,-.62$ versus $-.19,-.27$ respectively). This finding is also in accordance with the item content of CHIP factor NC.

The findings clearly support the construct validity of CHIP factors DM and NC. For factor VC, further validity assessment will be necessary.

Table 4.IV. Criterion-related validity for CHIP factors DM, VC and NC: Pearson product-moment correlation coefficient with measures of health behaviors 'walking', 'bicycle riding', 'standing', and 'sitting'.

\begin{tabular}{lllll}
\hline CHIP-factors & walking & bicycle & standing & sitting \\
\hline CHIP-DM & $-.51^{* *}$ & $-.60^{* *}$ & $-.37^{*}$ & -.31 \\
CHIP-VC & .07 & -.11 & -.30 & $-.38^{*}$ \\
CHIP-NC & -.19 & $-.38^{*}$ & -.27 & $-.62^{* *}$ \\
\hline
\end{tabular}

${ }^{*} \mathrm{p}<0.01,{ }^{* *} \mathrm{p}<0.001$ (one-tailed) 


\subsection{Study VI: validity of CHIP-N and CHIP-D}

Study VI examined the relationship between CHIP factors $N$ and $D$, and relevant self-report measures. Although there are many studies in the pain assessment literature in which intercorrelations are investigated between self-ratings and observations, such correlations do not "validate" any of the measures. Nevertheless, if significant correlations emerge, they might support the labeling of the CHIP factors.

\subsubsection{Subjects}

In this study, the subjects were the same as those involved in study III.

\subsubsection{Procedure}

As no reliable Dutch observational instruments were available as concurrent measures for CHIP factors $N$ and $D$, it was decided to choose relevant selfreport measures. Following measures were selected:

a. The MMPI-Depression Scale (MMPI-D; Hathaway \& McKinley, 1967) is considered a valid self-rating of depression.

b. The Welsh Anxiety Scale (WAS; Welsh, 1965), derived from the MMPI was considered as a general measure of nervousness.

The observation occurred as described in study II. For this study, however, only the data of the second observation day are used. At this moment the MMPI was also available, of which the MMPI-D scale and the WAS raw scores were calculated. Correlations were calculated among these self-report measures and the CHIP factors.

\subsubsection{Results and discussion}

The differential pattern of significant correlations is shown in Table $4 . \mathrm{V}$. Significance emerged only for CHIP factors $N$ and $D$, suggesting that these factors indeed capture the observational component of depression and nervousness. The significant correlation between CHIP factor N and MMPID might also be due to the existing correlation between MMPI-D and WAS. Therefore partial correlations were calculated, controlling for WAS and MMPI-D respectively. Significance of the correlations emerged only in the hypothesized direction, thereby confirming the validity of $\mathrm{CHIP}$ factors $\mathrm{N}$ and D.

These results, however, should be regarded with caution. As a different kind of assessment is used (observation versus self-report), lower corrrelations might be expected than in the case where only self-report or only observational measures are correlated (Gannon \& Haynes, 1986). 
Table 4.V. Criterion-related validity for CHIP factors $N$ and D: Pearson product-moment correlation coefficients $\left(r_{x y}\right)$ and partial correlations $\left(r_{x y z}\right)$ among CHIP factors and self-report measures MMPI-Depression and WAS.

\begin{tabular}{lllll}
\hline & MMPI-D & \multicolumn{3}{l}{ WAS } \\
& $\mathrm{r}_{x y}$ & $\mathrm{r}_{\mathrm{xy}-\mathrm{z}}$ & $\mathrm{r}_{\mathrm{xy}}$ & $\mathrm{r}_{\mathrm{xy} \cdot \mathrm{z}}$ \\
\hline CHIP-DM & .23 & .22 & .07 & -.01 \\
CHIP-VC & .23 & .09 & $.37^{*}$ & .31 \\
CHIP-NC & .24 & .13 & .30 & .25 \\
CHIP-N & $.45^{* *}$ & .32 & $.49^{* *}$ & $.41^{*}$ \\
CHIP-D & $.47^{* *}$ & $.42^{*}$ & .27 & .07 \\
WAS & & & & \\
\hline
\end{tabular}

" $\mathrm{p}<0.01,{ }^{* *} \mathrm{p}<.0 .001$ (one-tailed)

\subsection{General discussion}

Based on the results of the study described in the previous chapter, in which the components of observed chronic pain behavior were identified, the present study offered an operationalization of the broad interpersonal pain behavior' construct in the form of the CHIP. This paper also examined the psychometric properties of this new observational instrument. Both reliability and validity of the main factors appear promising.

Unlike self-reports of the pain experience, which rely on the patient him/herself to provide data, the CHIP represents a global rating scale that provides data resulting from direct observation. The observation occurs in a therapeutic environment and can be made by nurses who frequently interact with the patient outside structured therapy sessions. The advantages of the CHIP are that it is based on empirically derived items, that it is relatively short, and easy to use. Because of its simplicity, the CHIP might be suitable for its use by the patient's spouse in the home environment as well.

Compared to existing observation methods, the CHIP might provide a more comprehensive picture of the pain patient. A recent meta-analysis of non-medical treatments for chronic pain indicated that psychological treatments reliably affect mood and subjective symptom ratings (Malone \& Strube, 1988). The CHIP offers two observational measures related to mood (nervousness and depression). 
One disadvantage is that a certain degree of inference is required to complete a rating. The rater must observe the patient and, through inference, determine which rating is most appropriate. Moreover, the CHIP, like most global rating scales, may be susceptible to observer bias. As a consequence, explicit training, during which a consensus must be reached with regard to the specific meaning of the individual items, is a necessary prerequisite for its application. Finally, the CHIP has not yet been examined with regard to its sensitivity as a measure of patient progress during a pain management program. A weakness of this study concerns the results of study $\mathrm{V}$, which do not fully support the validity of the factors 'nervousness' and 'depression'. A similar study, but based solely on observational concurrent measures (when they become available) is recommended. Furthermore, CHIP factor 'verbal complaints' awaits further validation.

In recent publications (Syrjala \& Chapman, 1984; Turk \& Flor, 1987; Vlaeyen et al., 1989) researchers have stated that it is necessary to establish the presence or absence of respondent, operant, and cognitive contributions to the pain problem, and that treatments should be based on these 'patient profiles'. In spite of its disadvantages, the CHIP may be a useful instrument in identifying chronic pain patients whose pain problem can be characterized as being mainly overt-motoric, as compared to more cognitive or physiological pain problems. During a pre-treatment baseline observation period, high scores on the CHIP may indicate that the patient is a good candidate for an operant pain management program aimed at reducing pain behaviors and restoring healthy behaviors. Furthermore, many disadvantages can be offset and the advantages retained if multiple measures are employed. 



\section{CHAPTER 5}

\section{Reliability and validity of a Dutch version of the pain behavior scale}

\subsection{Introduction}

Until now only a limited number of observation scales have been developed that map the observable pain behavior. These are the Audiovisual Taxonomy of Assessing Pain Behavior (ATAPB, Keefe et al.,1982,1987), the Checklist for Interpersonal Pain behavior (CHIP, Vlaeyen et al.,1989) and the UAB Pain Behavior Scale (PBS, Richards et al,1982). The ATAPB employs video-recordings of patients performing a number of motor activities. The video sessions are divided into segments of 20 seconds and using five categories the frequency of occurrence of pain behaviors is determined. The disadvantage of this accurate method is that it is difficult to bring into therapeutic practice. The CHIP is based on empirically derived components of observed chronic pain behavior (Vlaeyen et al., 1987).

The PBS is possibly the observation scale that is most rapidly completed and consists of a list of 10 pain behaviors of which the frequency or intensity of occurrence can be registered using a 3-point assessment scale (Table 5.1). The total PBS score is the sum of the ratings of the individual items. Eight items pertain to pain behavior in a communicative sense. These are observed during a 5-minute observation procedure during which patients are asked to walk, stand up, move from a sitting to a standing position and vice versa. The other two items pertain to the use of medication and to the amount of time lying down because of pain. The study by Richards et al. (1982) indicates that both the inter-rater reliability $(0.95, \mathrm{p}<0.01)$ and the intra-rater reliability of the total score over two consecutive days $(0.89$

* This chapter is a translated and slightly modified version of Vlaeyen, J.W.S., Pernot, D.F.M., Kole-Snijders, A.M.J., Schuerman, J.A., Van Eek, H., \& Groenman, N.H. (1990). Betrouwbaarheid en validiteit van een Nederlandse versie van de Pain Behavior Scale (PBS). Nederlands Tijdschrift voor de Psychologie, 45, 184-189. 
$, \mathrm{p}<0.01)$ is amply sufficient. The internal consistency was not calculated. The validity study was limited to the study of the relationship with selfreport measures of the pain experience and with measures of healthy behavior.

Nonsignificant correlations were found with the pain-intensity score of the McGill Pain Questionnaire (Melzack,1975) and the Visual Analog Scale $(0.17$ and 0.16 respectively). Some significant, but moderate negative correlations were found between the PBS and activities such as walking, cycling, sitting and standing $(-.30,-.10,-.29$ and -.38 respectively).

The PBS' main merit is possibly its practical utility, the quick scoring and the reasonable reliability which makes it a fitting instrument for conducting repeated measurements in an outpatient setting (Feuerstein et al.,1985). Disadvantages of the PBS are that the choice of pain behaviors is not based on empirically derived taxonomy, and that its validity has not yet been fully determined. Moreover, the total score consists of the sum total of the individual item scores in which scales are used that are not the same for all items. Finally, the validity of the Dutch version has not been studied yet.

Five studies will be presented. The first study examines the characterisctics of the Dutch version of the PBS (Pijngedrag Schaal, PGS), including the internal consistency. Two studies focus on the intra-rater reliability and the third study on the inter-rater reliability. Study IV examines the validity of the PGS. All of the studies were carried out with chronic low back pain patients admitted to an in patient rehabilitation program at the Department of Rheumatology of the Lucas Foundation for Rehabilitation in Hoensbroek, The Netherlands. In those instances where correlations have been calculated, Kendall's correlation coefficient Tau has been used because of the relatively small sample sizes. An exception is made for the calculation of the internal consistency, for which the SPSS-PC+ V2.0 Reliability Analysis package was used (SPSS Inc,1988).

\subsection{Characteristics of the Dutch version (PGS).}

Table 5.I shows the original American version of the PBS and the translated Dutch version PGS. The translation was based on the meaning of the individual items described by Richards et al. (1982).

\subsubsection{Method}

The observations were made during a standardized procedure for the assessment of a number of motoric skills: standing, sitting, walking, cycling 
Table 5.I. De original PBS and the Dutch version PGS.

\section{PBS}

1. Vocal complaints: Verbal none occasional frequent

2. Vocall complaints: Non-verbal.

(moans, groans, gasps, etc.)

none

occasional

frequent

3. Down-time:

(time spent lying down per day

because of pain: 8 a.m. -8 p.m.)

none

$0-60 \mathrm{~min}$.

$>60 \mathrm{~min}$.

4. Facial grimaces

none

mild and/or infrequent

severe and/or frequent

5. Standing posture

normal

milldy impaired

distorted

6. Mobility

no visible impairment

mild limp and/or mildly

impaired walking

marked limp and/or labored walking

7. Body language

(elutching, rubbing site of pain) none

occasional

frequent

8. Use of visual supportive equipment

(braces, crutches, cane, leaning

on furniture, TENS, etc.)

Do mot score if equipment prescribed.

none

occasional

dependent: constant use

9. Stationary movement

sits or stands still

occasional shifts of position

constant movement, position shifts

10. Medication

none

non-narcotic analgesic and/or

psychogenic medications

as prescribed.

demands for increased dosage or frequency, and/or medication abuse.
PGS

1. Verbaal pijngedrag

geen

incidenteel

frekwent

2. Niet-werbale wocale klachten

(grommen, steunen, kreunen, zuchten)

geen

incidenteel

frekwent

3. Liggen wegens pijn

niet

$0-60 \mathrm{~min}$.

$>60 \mathrm{~min}$.

4. Pijnlijke gezichtsuitdrukkingen

geen

mild/weinig

sterk/frekwent

5. Staande houding

normaal

mild gestoord

gestoord

6. Mobiliteit

niet gestoord

letwat hinkend, gestoord

duidelijk gestoorde gang

7. Lichaamstaal

(wrijven over pijnlijke plekken)

geen

incidenteel

frekwent

8. Gebruik van ondersteuning

(krukken, stok, leunen op meubilair,

TENS, etc.)

Niet scoren als steun is voorgeschreven.

geen

incidenteel

afhankelijk: continue

ondersteuning

9. Zitten

zit stil

incidentele positieverandering

constante positieverandering

10. Medicatie

geen

als voorgeschreven

wraagt verhoging dosering of frekwentie 
and performing rowing movements with arms and legs. During this procedure, the pain-rest contingency principle was used in the instruction: "Continue for as long as you can. Only stop when pain prevents you from continuing or when we give you a sign that you can terminate the activity." A trained research assistant observed pain behaviors during the performance of these motor tasks and filled in the PGS upon completion thereof, without being aware of the results of the motor tasks. For the 12 men, the mean age was 40.25 years (SD 7.7), the mean duration of pain complaints was 12.4 years $(S D=6.5)$ and they spent an average of 608 minutes lying down daily $(S D=170)$. The 23 women were, on average, 43.52 years old $(S D=7.2)$, had been troubled by pain complaints for 11.56 years $(S D=9.5)$ on average, and spent 703 minutes daily lying down. The differences between the men and the women for these variables are not significant. The PGS scores varied between 0.5 and 8.0, with an average of 3.97 and a standard deviation of 1.89. Although the average score for the women $(4.35, \mathrm{SD}=1.99)$ is higher than that of the men $(3.25, \mathrm{SD}=1.51)$, this difference is not significant. The PGS total score correlates significantly with age (Kendall's correlation coefficient $\mathrm{Tau}=.21, \mathrm{p}<0.05$ ).

Table 5.II. Characteristics of the PGS.

\begin{tabular}{|c|c|c|c|c|c|}
\hline \multirow[t]{2}{*}{$\begin{array}{l}\text { PGS } \\
\text { Items }\end{array}$} & \multicolumn{2}{|c|}{$\begin{array}{l}\text { Intra-rater } \\
\text { reliability }\end{array}$} & \multirow{2}{*}{$\begin{array}{l}\begin{array}{l}\text { inter-rater } \\
\text { reliability }\end{array} \\
\mathbb{I I I}\end{array}$} & \multirow[t]{2}{*}{$\begin{array}{l}\text { item-total } \\
\text { correlation }\end{array}$} & \multirow[t]{2}{*}{$\begin{array}{l}\text { alpha when } \\
\text { item deleted }\end{array}$} \\
\hline & $I$ & III & & & \\
\hline 1 & $0.72^{* * *}$ & $0.50^{* * * *}$ & $0.62^{* * *}$ & 0.45 & 0.73 \\
\hline 2 & $0.61^{* *}$ & $0.37 *$ & $0.61^{* * *}$ & 0.33 & 0.74 \\
\hline 3 & 0.27 & & & 0.41 & 0.73 \\
\hline 4 & $0.66^{* * *}$ & $0.59 * *$ & $0.64 * * *$ & 0.63 & 0.70 \\
\hline 5 & $0.80^{* * *}$ & $0.64^{* * *}$ & $0.56^{* * * *}$ & 0.58 & 0.71 \\
\hline 6 & $0.80^{* * *}$ & $0.64 * *$ & 0.71 ** & 0.50 & 0.72 \\
\hline 7 & $0.74^{* * * *}$ & $0.43^{* *}$ & $0.43^{* * 4 *}$ & 0.36 & 0.74 \\
\hline 8 & $0.97^{* * * * * *}$ & $0.83^{*+* 4 *}$ & $0.44^{* * *}$ & 0.51 & 0.72 \\
\hline 9 & 0.71 *** & $0.29 *$ & 0.17 & 0.36 & 0.74 \\
\hline 10 & $0.38^{*}$ & & & -0.01 & 0.78 \\
\hline tot & $0.83^{* * *}$ & $0.64 * * *$ & $0.74^{* * *}$ & & \\
\hline
\end{tabular}

${ }^{*} p<0.05,{ }^{* *} p<0.01, * * * p<0.001$

I, II, III: data from study I, study II, and study III (Kendall's correlation coefficient Tau). 


\subsubsection{Results}

Table 5:II shows the characteristics of the PGS. The internal consistency of the PGS (Cronbach's alpha) equals 0.75. The item-total correlation coefficients and Cronbach's alpha if one of the items is omitted, are shown in Table 5.II. It is noteworthy that item 10 does not correlate with the total score. Upon omission of this item the internal consistency increases. Alpha then becomes 0.78 .

\subsection{Intra-rater reliability: Study I}

The intra-rater reliability was studied using the test-retest method. In study I the PGS was rated by nurses. In study II the observation was done by research assistants.

\subsubsection{Method.}

The group consisted of 14 male and 6 female chronic low back pain patients. The observation was performed twice by one of three trained nurses and took place at two moments during the first and second week of admittance, respectively. During the first two weeks of admittance baseline data were obtained and no treatment occurred. Kendall's correlation coefficient Tau between the two moments was calculated for each separate PGS item and for the PGS total score.

\subsubsection{Results.}

As can be seen in Table 5.II, significant correlations were found for most PGS items, except for item 3. Among the other items, item 10 demonstrated the lowest correlation. These results suggest that the PGS total score has sufficient temporal stability. For item 3 the temporal stability is insufficient. Based on this fact and the absent item-total correlation for items 10, the decision was made to remove items 3 and 10 from the PGS. As was mentioned before, both items concern pain behavior that is impossible to observe over a brief period of time. Therefore, their omission does not produce an essential loss of information. From hereon, PGS denotes the observation scale consisting of the eight remaining items.

\subsection{Intra-rater reliability: Study II}

\subsubsection{Method}

This study concerned the group $(n=35)$ of patients described in paragraph 
5.2. The procedure for the collection of baseline data was repeated 2 weeks later. Kendall's correlation coefficient Tau was calculated between the PGS scores of the two measuring points.

\subsubsection{Results}

Table 5.II shows significant, but moderate correlations for all items and for the total score, which ind icates that the reliability can be deemed reasonable.

\subsection{Inter-rater reliability: Study III}

\subsubsection{Method}

In this study, the subject sample consisted of 37 female and 10 male chronic low back pain patients. The working schedule of the nurses did not allow the same pair of nurses to observe the patients. Therefore the inter-rater reliability was performed by two trained research assistants who, independently of each other, observed the patients during an approximately 30-minute period and then completed the PGS. The observation took place while the patients carried out standardized activities as described in paragraph 5.2. Kendall's correlation coefficient Tau was calculated for the PGS scores of both observers.

\subsubsection{Results.}

The data in Table 5.II demonstrate that significant correlations for the individual PGS items are found, except for item 9 . Items 7 and 8 demonstrate insufficient correlation. For the total score however, a fairly high, significant correlation is found. The conclusion can be drawn that the inter-rater reliability of the PGS is sufficient. Only the total score was involved in the further analyses.

\subsection{Validity: Study IV}

In the previous chapter, it was shown that all correlations among the PGS and ATAPB categories (Keefe et al., 1987) were significant (Table 4.III, p. 77). This supports the strong association between the PGS and the number of pain behaviors that can be counted using video recordings, underscoring the validity of the PGS. To further examine the validity of the PGS, this study investigated the relationship between PGS scores and the frequency of a number of healthy behaviors such as the motor activities of standing, sitting, walking, cycling and leg-rowing. Because healthy behavior 
is known to be incompatible with pain behavior, negative correlations were expected.

\subsubsection{Method}

This study concerned the patient group $(n=35)$ as described in paragraph 5.2. Kendall's correlation coefficient Tau was calculated between the PGS total score and the scores on the motor tasks.

\subsubsection{Results}

Table 5.III demonstrates a significant negative correlation between the PGS score and the scores on the motor tasks. These data confirm the validity of the PGS.

Table 5.III. Kendall's correlation coefficient Tau among PGS total score and the motoric activities standing, sitting, walking, swimming, bicycling. and leg-rowing.

\begin{tabular}{llllll}
\hline & Standing & Sitting & Walking & Bicycling & Leg-rowing \\
\hline PGS total & $-.41^{* * *}$ & $-.38^{* *}$ & $-.46^{* * *}$ & $-.42^{* * *}$ & $-.26^{*}$ \\
\hline
\end{tabular}

${ }^{*} \mathrm{p}<0.05,{ }^{* *} \mathrm{p}<0.01,{ }^{* * *} \mathrm{p}<0.001$

\subsection{Discussion}

The PGS proves to be a quickly and simply scored observation scale for pain behavior which is easily applicable to clinical therapeutic practice. The PGS can be used by both nurses and research assistants. Scoring of 8 items is possible after a relatively brief observation period in which the patient performs a number of motor activities. For the two remaining items (lying down due to pain and use of medication) a longer observation period is necessary. The reliability of these 2 items is insufficient. Removal of these items from the scale improved the internal consistency (Cronbach's alpha increased from .75 to .77 ). These results led to the use of the PGS total score only, based on 8 reliable items, for the validity studies. These items were the same ones that Feuerstein et al. (1985) maintained in their version of the PBS that had been modified for outpatient use. The validity of the PGS can be deemed satisfactory. In accordance with expectations the PGS correlates positively with the precise counts of pain behaviors using the 
ATAPB, and negatively with the frequency of a number of healthy behaviors, in casu motoric activities.

The PGS is, as all observation scales, sensitive to 'observer bias". Explicit training during which the observers attain a consensus upon the meaning of the items and the scoring alternatives must precede the use of the PGS. The conclusion can be drawn that the PGS, based on the 8 reliable items, is a valuable instrument that fills a gap in the currently available pain measuring instruments. 


\section{Behavioral assessment of residual health behavior in chronic low back pain patients through the pain-rest contingency principle ${ }^{*}$}

\subsection{Introduction}

This chapter focuses on assessment within the overt-motoric response system of chronic pain. Ideally, this assessment includes measurement of both behavior excesses and behavior deficits. Behavior excesses refer to behaviors that occur too frequently or too strongly, and that hamper the occurrence or the maintenance of healthy behavior. Overt pain behavior, of which the components and dimensions have been described in chapter 3 is considered a behavior excess. An instrument to assess these components is described in chapter 4.

Behavioral deficits refer to those behaviors which do not belong to the person's behavioral repertoire anymore, or less frequently as needed, and which appear in healthy and pain-free people. With regard to behavioral deficits, at least two possibilities should be considered:

a. The pain problem, and the avoidance of physical activities, may have existed so long that a substantial skill deficit for normal physical and social activities has developed. This process is also referred to as the 'disuse syndrome' (Bortz, 1984).

b. Although the patient is physically able to perform healthy behaviors, he or she has learned that avoidance of physical and social activities reduces the amount of suffering. The reasons that have been hypothesized in

* Parts of this chapter have been presented by Snijders, A.M.J., Vlaeyen, J.W.S., Van Eek, H., Groenman, N.H., \& Schuerman, J.A. (1990) at the IASP VIth World congress on Pain, Adelaide, Australia, and sponsered by the Stichting Wetenschappelijk Onderwijs Limburg. Abstract: Pain, supplement 5, S 394. 
relation to why some pain patients avoid and others do not avoid physical and social activities when experiencing pain have been discussed in paragraph 2.3.1.

Consequently, the assessment of behavioral deficits should take both processes into account. For the extent to which deficits can be explained as a result of avoidance, the assessment procedure can best be conceptualized as a Behavioral Avoidance Test (BAT). A BAT is a behavioral measure, where a fear-eliciting (or pain-eliciting) stimulus is placed in a standardized environment and a person is instructed to approach the stimulus and engage in progressively more bold interactions with it (Bellack \& Hersen, 1988). Although BATs have been extensively used in fear and anxiety assessment, their application in the area of chronic pain has not been described yet. $A$ BAT for pain patients would be based on the pain-rest contingency principle: patients are asked to perform an activity until pain causes him or her to wish to stop.

Second, for the extend the behavioral deficits are the result of a substantial skill dleficit, their assessment is similar to the assessment of residual healthy behaviors, or residual motoric skills.

Instruments that have been developed to assess residual healthy behaviors in CLBP are those that examine motoric functional status or disability. These instruments can be divided into four groups: 1 . self-report measures, 2. quantitative measures of strength, 3. a combination of subjective and objective measures, and 4 . comprehensive functional evaluation.

1. The first group is composed of self-report measures, questionnaires in which the patient describes his limitations in performing various activities of daily living. An example of this type of measure is the Oswestry Low Back Pain Disability Questionnaire (Fairbank, 1980) and the recently developed Functional Assessment Screening Questionnaire (Millard, 1989). Visual Analog Scales (VAS) also belong in this group; on these scales, patients mark a point on a straight line whose extremes represent the extreme answers to questions concerning functional status. Measuring the distance of a patient's mark along the line gives an index of the severity of the disability. An important limitation of these methods concerns the use of the self-report format. The drawbacks of assessing pain behavior through the use of self-report instruments have been documented by Kremer et al. (1981). The findings resulting from self-report measures may correlate more closely with general distress than with the activities a person actually performs, not to mention the effects of social desirability and defensiveness. 
2. The second group of studies uses quantitative measurements of the maximum strength and fatigue behavior of the abdominal and paraspinal muscles (Langrana \& Lee, 1984). People with weak trunk muscles are supposed to be prone to back pain. Other investigators (Mayer et al., 1984, 1986) measure spinal range-of-motion, dynamic trunk strength, cardiovascular fitness, gait speed, obstacle course, static lifting, lifting underload and global effort to assess disability and to measure progress in a rehabilitation program. These measures are quite extensive and are not based on the pain-rest contingency principle.

3. The third group of studies (Million et al., 1981) uses a combination of subjective measurements (VAS for the severity of the complaint and interference with activities of daily living) and objective measurements such as straight leg raising, extension and flexion of the back. The drawbacks of the subjective measures have already been mentioned.

4. The fourth group of studies uses a comprehensive functional evaluation, including self-report questionnaires, objective measures, and observation scales. Naliboff et al. (1985) have developed the Physical Abilities Test (PAT), which includes observation scales for walking, lifting, and executing daily tasks. The PAT items were designed to measure a subject's maximum performance during which pain behavior and hesitancy in performance were specifically ignored. These tests are designed to assess maximum physical abilities, and not avoidance behavior.

It is interesting that none of the existing instruments includes swimming tasks even though swimming is considered to be a recommended back exercise (Krämer, 1981). Swimming is considered a healthy behavior that is being encouraged or shaped in many pain rehabilitation centers as an important part of the treatment program. So far, no instrument has been developed that can both assess swimming performance in chronic low back pain patients and be used to evaluate treatment outcome.

This chapter describes the development of an assessment instrument, designed as a BAT, which focuses on quantifying relevant behavioral deficits, or residual health behaviors, of CLBP patients. Besides psychometric requirements, such an instrument should meet at least following criteria:

1. The instrument should not be based on a self-report format.

2. The instruction should allow the patient to decide himself when to avoid the situation or the task: "continue until pain or suffering makes you terminate the task.". In this way, demand characteristics of the test situation are both minimized and held constant. Moreover, it is helpful to be able 
to capture the patient's behavioral deficits as closely as possible to the way they are occurring in his dally life.

3. The instrument must be highly standardized, and easily applicable in a clinical setting.

Several experiments will provide data regarding the inter-rater and testretest reliability, discriminative value, and validity of this instrument.

\subsection{Pillot study: The Motoric Skills Observation Scale (MOSOS).}

Prior to the construction of this assessment instrument, a list of possible tasks was formed by a group of eight physiotherapists, occupational therapists and sports therapists. The list concerned activities that were regularly used to assess the functional abilities of inpatients in the Rehabilitation Center at Hoensbroek, The Netherlands. For the purpose of this study activities were selected based on the above mentioned criteria. Special care was given to the standardization of the task in order to improve reliability. The activities chosen were grouped as follows: activities involving cyclic movements with the extremities, activities involving non-cyclic movements, and swimming. These three groups of activities were subsequently chosen to represent the items of the three parts of the Motoric Skills Observation Scale (MOSOS): MOSOS I, II, and III.

\subsection{Development of the MOSOS-I}

This test includes four items that represent activities concerning cyclic movements: walking, bicycling, arm rowing, and leg rowing. For all MOSOSI items the same instruction is used: "go on until pain prevents you from continuing.", and all items are modeled by the instructor. Special checklists are provided to record the qualitative aspects of the performance. The walking item consists of a normal speed walk on a measured course in the testing room. The distance (WALKD) and time spent walking (WALKT) are scored separately. For the bicycle item, an electronic bicycle ergometer (Laméris, type RH-30, Lade B.V. Groningen, The Netherlands) is used. The person is required to keep up a constant speed of 60 revolutions/minute with a power of $100 \mathrm{Watt}$. The time spent biclycling is recorded (BIKET). For both the arm rowing and leg rowing tasks, a simulating model of a rowing boat is used (Feelgood, Health Equipment B.V., Berlicum, The Netherlands). The person sits on a sliding seat. During the arm rowing task 
the person has to pull back two handles, one in each hand, which are connected with a weight of $5 \mathrm{Kg}$. The leg rowing task consist of pushing away with both feet a weight of $15 \mathrm{~kg}$. Both the amount of arm and leg rowing movements (ARMR and LEGR) as well as the time spent rowing (ARMRT and LEGRT) are recorded separately. A total time score (TSCORE) consists of the sum of the time scores of the four tests. The higher TSCORE, the less avoidance and the higher tolerance. For walking and bicycling a maximum time of 7 minutes is set. For the rowing tasks a the time limit is set to 5 minutes, allowing a complete administration within 30 minutes. The MOSOS-I is scored by a physiotherapist or by a trained research assistant.

\subsection{Development of the MOSOS-II}

Three items were chosen that represent activities that require a minimal amount of cyclic movements: sitting, standing, and replacing objects. For the sitting and standing tasks, the person may choose an activity (reading, solving puzzles, drawing etc.) while sitting and standing. For replacing objects 10 objects of varying size and weight are placed on a desk. Two meters from the desk two shelves are placed, a low one $(30 \mathrm{~cm}$ from ground) and a higher one (eye level). The person is asked to take the first of the numbered objects and put it on the lower shelf. The second object is to be placed on the higher shelf and so on. The desk and the higher shelf are adjusted according to the lenght of the person. The instruction for all MOSOSII items is standardized: "go on with the activity until pain prevents you from continuing." For each test, the time is recorded (SITT, STANDT and REPLACT). A maximum time is set to 25 minutes for sitting and standing, and 5 minutes for replacing objects. For the replacing objects test the number of objects successfully replaced is recorded as well (REPLACN). During the tests, qualitative aspects of performance are observed and recorded by means of checklists that are designed for each test separately. The test is scored by an occupational therapist or a trained research assistant.

\subsection{Development of the MOSOS-III}

The initial item pool for the development of the MOSOS-III was a list of 31 swimming tasks used by sports therapists at the Rehabilitation Center of the Lucas Foundation for Rehabilitation (Hoensbroek, The Netherlands). Because of their complexity, 10 items were eliminated. These items called 
for the use of two different swimming strokes at the same time (e.g. swimming breast stroke with the arms and crawl with the legs). The remaining 21 items served as the basic item pool for the development of the MOSOSIII. These items varied considerably in difficulty. Scoring of the items was only possible when the relative difficulty could be quantified. This first experiment was set up to establish weighted loadings for each item, based on the judgments of a group of therapists.

\subsubsection{Subjects}

Fifteen physiotherapists and sports therapists from the Lucas Foundation for Rehabilitation, Hoensbroek, served as subjects in this study.

\subsubsection{Procedure}

The procedure that led to the development of the FASS consisted of four consecutive steps.

In the first step, each therapist received a sheet of paper with a line, 60 centimeters long, on it. Two points of reference were marked on the line. At point 0, an easy task, 'standing in the water', was placed. At the other end of the line, the point 100 was marked by the task 'swimming the length of the swimming pool under water', a task considered to be very difficult. The 20 items, printed on small cards, were presented to the 15 physiotherapists and sports therapists with the following instruction:

"You receive 20 cards, on each of which a swimming task is printed. Please arrange these cards along this line according to their difficulty. You can designate differences in difficulty by the order of the cards and by the distances you leave between them."

When all therapists had individually arranged the cards along the line, the distance in centimeters of each card to the zero was determined for each therapist separately. The magnitude of the distance reflects the difficulty level of the 20 items, for which the mean distance in $\mathrm{cm}(\mathrm{M})$ and standard deviation in $\mathrm{cm}$ (SD) were calculated.

In the second step, agreement among the 15 therapists was determined through calculation of Cronbach's alpha, the widely accepted measure for internal consistency. In favor of internal consistency among therapists, data from therapists who deviated too much from the average judgment were excluded.

Thirdly, tasks with variances that are significantly greater than the variances of the majority of the tasks were eliminated.

The fourth step consisted of forming homogeneous groups of swimming 
tasks that could be assigned the same weight. A Hierarchical Cluster Analysis (HCA), using the complete linkage method, was applied to the remaining items. For each cluster, the mean difficulty was determined, which for practical reasons was subsequently transformed into a value on a scale ranging between 0 and 10 .

\subsubsection{Results}

Cronbach's alpha was .97 , suggesting excellent agreement among the 15 therapists. Deletion of the data of any therapist did not increase the level of alpha. F-tests, testing differences in variances of judgements for each of the 20 swimming tasks, revealed that variances of tasks $14,15,16$ and 19 were significantly higher than those of sixteen $(80 \%)$ other tasks ( $p<.01)$. For example, task 14 was placed at $5 \mathrm{~cm}$ from zero by one therapist and at 56 $\mathrm{cm}$ from zero by another therapist. Therefore they were removed from the MOSOS-III.

The dissimilarity matrix (therapists $X$ tasks) was subsequently submitted to HCA, using the complete linkage method. Inspection of the fusion coefficients revealed that the solution in 9 clusters was the most appropriate. Based on the item content of each cluster, listed in Table 6.I, the clusters were labeled as:

1. holding head under water, 2. floating, 3. partial backstroke, 4. total backstroke, 5 . side roll, 6 . breast stroke, 7 . tumbling, 8 . swimming under water, and 9. crawl. For each cluster, mean difficulty was calculated resulting in the following values (in $\mathrm{cm}$ ): $5.18,14.17,22.41,25.82,27.00,28.75,45.98$, 48.73 , and 48.79 . Subsequently, weighted scores ranging between 0 and 10 were assigned to each of the clusters by deviding the mean difficulty by 6 . For practical reasons these scores were rounded off, resulting in the following scores: $1,2.5,3.5,4.5,4.5,5,7.5,8$, and 8 .

The instruction of this new instrument is based on the pain-rest contingency principle: "Continue the task until pain, fatigue or physical discomfort prevents you from continuing." For each task a maximum time limit is set. Tasks are presented with increasing levels of difficulty. Three different scores can be calculated: (1) a total score (totscore), which is the sum of the weighted scores of the successfully performed swimming tasks; (2) a time score (tscore), which is the total time the patient was able to swim; and (3) a distance score (dscore), which is the total distance the patient swam. The time and distance scores are based on the three forms of back stroke and the two breast strokes. The maximum time provided to each subject for each swimming stroke was 3 minutes, so that the whole test could be completed within 30 minutes. 
Table 6.1. Hierarchical cluster analysis of swimming tasks, including mean difficulty of individual tasks, cluster means $(M)$ and standard deviation (SD).

CLUSTER

1. Holding head under water.
SWIMMING TASK

5. Holds onto the edge of the swimming pool and brings head under water.

11. Blows bubbles under water

1. Brings the head under water without holding onto the edge of the swimming pool.

$6.51 \quad 3.38$

\section{$5.2 \quad 2.10$}

2. Floating

17. First floats on the back and then stands upright.

20. Floats on the chest and then stands upright.

3. Partial backstroke

4. Swims back stroke only with the legs.

22.4

6.48

4. Total backstroke

18. Swims the back stroke with symmetrical arm movements half under water.

6. Swims the back stroke with symmetrical arm movements completely under water. 26.4

25.8

5. Side roll

7. Floats on the chest and turns to the back via the longitudinal axis.

$27.0 \quad 9.10$

6. Breast stroke

8. Swims breast stroke with the head above the water.

13. Swims the breast stroke with the head in the water, breathing every third stroke

7. Tumbling

10. Floats on the chest and turns forward to the back.

2. Floats on the back and turns back to the chest.

8. Swimming under water

9. Swims under water for 7 meters.

48.7

9.82

9. Crawl

12. Swims the back crawl.

3. Swins the crawl.

$46.9 \quad 10.66$

$50.7 \quad 7.02$

48.8 


\subsection{Characteristics of the MOSOS-scores}

The following study evaluates the intercorrelations among the scores of the three parts of the MOSOS, tests the scores distribution against the normal distribution, and examines the relationship between the MOSOS scores and individual differences including sex, age, and duration of pain complaint.

\subsubsection{Subjects}

Fourty-one CLBP patients who were admitted for an inpatient operant treatment program participated in this experiment. These patients were classified by their physicians as either having minimal organic findings or displaying pain complaints disproportionate to the demonstrable organic basis of their pain. The 29 female and 12 male patients, with mean ages of 44.8 years $(S D=7.6)$ and 40.2 years $(S D=7.7)$ respectively, took the MOSOS prior to entrance into a treatment program aimed at reactivation similar to the operant treatment described in Appendix A. The mean duration of the pain complaints was 12 years $(S D=10.3)$ for the female subgroup and 12.4 years $(S D=6.5)$ for the male subgroup. The female patients were more disabled than the male patients. In our sample female patients had more surgery for back pain than the male group $(t=-2.72, p=.011)$ and the female group made more use of supportive equipment than the male group; $31 \%$ of the female group and $17 \%$ of the male group used supportive equipment. For practical reasons, the MOSOS-III was always the first assessment instrument in a more extensive pain assessment procedure. The sequence by which the MOSOS-I and the MOSOS-II were taken occurred randomly.

\subsubsection{Procedure}

For the total time score of MOSOS-I, internal consistency is calculated (Cronbach's Alpha). For each score, skewness of the distribution was calculated. Irrespective of these results, non-parametric statistical analyses were used because of the relatively small sample size. Kendall correlation coefficient Tau was calculated among MOSOS scores, and among MOSOS scores, age, and duration of pain complaints. A Mann-Whitney U test was carried out in order to examine whether the female and male scores differed significantly.

\subsubsection{Results}

Internal consistency of TTSCORE was sufficient (Alpha $=0.65$ ) to consider it as a separate composite score. Tables 6.II, 6.III and 6.IV present the intercorrelations for the three parts of the MOSOS separately. For the MOSOS- 
I and MOSOS-III items, significant positive correlations exist suggesting high internal consistency. This is not the case for the MOSOS-II which includes items that are not significantly correlated with each other. The MOSOS-II appears to measure more than just one construct.

Table 6.V. shows that most of the MOSOS scores are quasi-normally distributed. This is not the case for ARMR and REPLACT, probably because too often the limit was reached. Table $6 . \mathrm{V}$. further shows that generally a negative correlation exists between age and residual health behavior. Surprisingly, no correlation exists between residual health behavior and duration of pain complaints.

Table 6.II. Intercarrelations of MOSOS-I-items

\begin{tabular}{lllll}
\hline & 1 & 2 & 3 & 4 \\
\hline 1. WALKD & 1.0 & $0.34^{* * *}$ & $0.44^{* * *}$ & $0.62^{* * *}$ \\
2. ARMR & & 1.0 & $0.47^{* * *}$ & $0.38^{* * *}$ \\
3. LEGR & & & 1.0 & $0.53^{* * *}$ \\
4. TTSCORE & & & 1.0 \\
\hline
\end{tabular}

${ }^{*} \mathrm{p}<.05{ }^{* *} \mathrm{p}<.01,{ }^{* 4 *} \mathrm{p}<.001$

Table 6.III. Intercorrelations of MOSOS-II-items

\begin{tabular}{lllll}
\hline & 1 & 2 & 3 & 4 \\
\hline 1. SITT & 1.0 & $0.21^{*}$ & 0.17 & -0.05 \\
2. STANDT & & 1.0 & 0.08 & $-0.29^{* *}$ \\
3. REPLACN & & & 1.0 & $-0.21^{*}$ \\
4. REPLACT & & & 1.0 \\
\hline
\end{tabular}

${ }^{*} \mathrm{p}<.05, * * \mathrm{p}<.01$

Table 6.IV. Intercorrelations of MOSOS-III-items

\begin{tabular}{llll}
\hline & 1 & 2 & 3 \\
\hline 1. TOTSCORE & 1.0 & $0.63^{* * *}$ & $0.72^{* * *}$ \\
2. TSCORE & & 1.0 & $0.78^{* * *}$ \\
3. DSCORE & & 1.0 \\
\hline
\end{tabular}

$p<.001$ 
Table 6.VI shows the mean scores and standard deviation for the male and female CLBP patients separately, and the results of the Mann-Whitney U test. Generally, male CLBP patients score significantly higher than female CLBP patients. Of interest is that for the MOSOS-II and MOVOS-III scores, these differences are less pronounced. A possible reason for the difference between the male and female performances might be that female patients are more disabled by the pain than the male patients: In our population female patients have had more surgery for back pain than the male group and the female group made more use of supportive equipment than the male group.

Table 6.V. MOSOS Characteristics: Skewness and relationship among MOSOS item scores, age and duration of pain complaints: Kendall Correlation Coefficient Tau.

\begin{tabular}{lll}
\hline Skewness age & $\begin{array}{l}\text { duration of } \\
\text { pain complaints }\end{array}$ \\
\hline
\end{tabular}

\section{MOSOS-I}

$\begin{array}{llll}\text { 1. WALKD } & 0.77 & -.26^{*} & .09 \\ \text { 2. ARMR } & 1.06 & -.20^{*} & .03 \\ \text { 3. LEGR } & 0.86 & -.19^{*} & .17 \\ \text { 4. TSCORE } & 0.55 & -.29^{* *} & .11\end{array}$

\section{MOSOS-II}

$\begin{array}{lccc}\text { 1. SITT } & -0.88 & -.25^{*} & -.05 \\ \text { 2. STANDT } & -0.11 & .07 & .07 \\ \text { 3. REPLACN } & -0.72 & -.24^{*} & -.03 \\ \text { 4. REPLACT } & 3.06 & .07 & -.07\end{array}$

\section{MOSOS-III}

1. TOTSCORE

2. TSCORE

${ }^{*} \mathrm{p}<.05 ;^{* *} \mathrm{p}<.01 ; * * * \mathrm{p}<.001$ 
Table 6.VI. Sex differences of MOSOS scores: Mann-Whimey U test.

\begin{tabular}{llllll}
\hline & $\begin{array}{l}\text { Female } \\
\text { Mean }\end{array}$ & SD & Male & & \\
Mean & SD & U \\
\hline
\end{tabular}

\section{MOsos-I}

$\begin{array}{lrrrrr}\text { 1. WALKD } & 147.6 & 114.6 & 317.8 & 142.7 & 76.5^{* *} \\ \text { 2. ARMR } & 47.4 & 38.8 & 96.0 & 53.6 & 74.0^{* *} \\ \text { 3. LEGR } & 47.2 & 39.6 & 88.2 & 49.4 & 80.0^{* *} \\ \text { 4. TSCORE } & 576.3 & 295.6 & 984.3 & 417.6 & 77.0^{* *}\end{array}$

\section{MOSOS-II}

$\begin{array}{lrrrrc}\text { 1. SITT } & 1086.5 & 452.1 & 1225.1 & 507.5 & 132.0 \\ \text { 2. STANDT } & 887.5 & 447.8 & 1093.4 & 441.0 & 128.0 \\ \text { 3. REPLACN } & 8.7 & 1.0 & 9.9 & 0.3 & 56.5^{* * *} \\ \text { 4. REPLACT } & 117.8 & 55.3 & 96.8 & 23.1 & 138.0\end{array}$

MOSOS-III

$\begin{array}{lrrrrr}\text { 1. TOTSCORE } & 27.5 & 24.0 & 46.6 & 19.6 & 89.5^{*} \\ \text { 2. TSCORE } & 355.8 & 303.6 & 512.7 & 236.2 & 119.0 \\ \text { 3. DSCORE } & 125.7 & 125.2 & 182.8 & 134.4 & 126.5\end{array}$

${ }^{*} \mathrm{p}<.05 ; * * \mathrm{p}<.01 ; * * * \mathrm{p}<.001$

\subsection{Reliability of the MOSOS}

In this study, both inter-rater reliability and test-retest reliability of the three instruments are examined.

\subsubsection{Subjects}

Two groups of subjects participated in this experiment: the group of 41 CLBP patients who also participated in the previous experiment and a group of 27 healthy and pain-free controls.

The healthy controls are selected from a group of people who responded positively to an announcement in a local newspaper. Fifteen female and 12 male subjects participated in the study. The subjects were medically screened before participation in the experiment, to exclude subjects with back pain or other pain problems. All 27 subjects were accepted. Their mean ages are 44.7 years $(S D=12.1)$ and 45.8 years $(S D=10.5)$, respectively. An ANOVA testing mean ages by group (CLBP vs. normals) and sex (male vs. female) 
revealed no significant differences (group: $F=1.99, \mathrm{p}=.164$, sex: $F=0.48$, $\mathrm{p}=.49$, group $x$ sex: $F=0.42, p=.52$ ).

Test-retest reliability was determined for the 41 CLBP patients. Interrater reliability was determined for the 27 healthy subjects.

\subsubsection{Procedure}

Two experimenters independently scored parts of the MOSOS for the healthy controls. Items for which it was assumed that the healthy controls would reach maximum scores were omitted: all MOSOS-II items and MOSOS-I walking. The Kendall correlation coefficient Tau between their scores was calculated.

The CLBP patients took the MOSOS twice, with an interval of two weeks. Both administrations occurred before entering the treatment program. Kendall correlation coefficients Tau were calculated between the scores on the two different occasions.

\subsubsection{Results}

Although the inter-rater reliability has not been examined for all MOSOS items, the results presented in Table 6.VII leave no doubt. The MOSOS can be considered a sufficiently reliable assessment instrument.

\subsection{Discriminative value of the MOSOS}

The MOSOS has been developed as part of a more comprehensive chronic pain assessment. The test must be able to differentiate between CLBP patients and healthy controls. In order to evaluate the discriminative value of the MOSOS, scores of a group of healthy, pain-free controls are compared with scores of a group of CLBP patients.

\subsubsection{Subjects}

The subjects described in the previous experiment also participated in this experiment.

\subsubsection{Procedure}

For all MOSOS items, the Mean and Standard Deviation were calculated for both subject groups. A Mann-Whitney $U$ test was carried out to determine the differences between the two groups. Comparisons were not possible for the test items that were omitted in the pain-free control group. The test is carried out for the male and female subjects separately. 
Table 6.VII. Reliability of MOSOS: Kendall Correlation Coefficient Tau $(N=35)$.

\begin{tabular}{lll}
\hline & $\begin{array}{l}\text { Inter-nater } \\
\text { reliability }\end{array}$ & $\begin{array}{l}\text { Intra-rater } \\
\text { reliability }\end{array}$ \\
\hline
\end{tabular}

\section{MOSOS-I}

1. WALKD

2. WALKT

$\begin{array}{ll}- & 0.84^{* * *} \\ - & 0.76^{* * *}\end{array}$

3. BIKET

$0.92^{* * *}$

$0.74^{* * *}$

4. ARMR

$0.97^{* * *}$

$0.78^{* * *}$

5. ARMRT

$1.00 * * *$

$0.64^{* * *}$

6. LEGR

$0.99 * * *$

$0.70^{* * *}$

7. LEGRT

$1.00^{* * * *}$

$0.65^{* * *}$

8. TSCORE

$0.75^{* * *}$

\section{Mosos-II}

1. SITT

2. STANDT

3. REPLACN

4. REPLACT

\section{Mosos-III}

1. TOTSCORE

2. TSCORE

3. DSCORE

*** $\mathrm{p}<.0001$
$0.72^{* 4 *}$
$0.75^{* * *}$
$0.97^{* * * * *}$
$0.78^{* * *}$

\subsubsection{Results}

The results of Table 6.VIII show that for the female subjects, healthy controls score higher than CLBP patients on all items. For the male subjects (Table 6.IX), normals score significantly higher than CLBP patients, except on MOVOS-III items. This can be due to the fact that our malle sample is far less disabled that our female sample. Nevertheless, the conclusion that the MOSOS has a excellent discriminative value appears justified. 
Table 6.VIII. Discriminative value of MOSOS-III and MOSOS-I: Comparison between female normals ( $N=15)$ and female CLBP patients $(N=23)$.

\begin{tabular}{|c|c|c|c|c|c|}
\hline$\therefore$ & $\begin{array}{l}\text { CLBP } \\
M\end{array}$ & $S D$ & $\begin{array}{l}\text { Norm } \\
\text { M }\end{array}$ & $\mathrm{SD}$ & $\mathbb{U}$ \\
\hline MOSOS-I & \multicolumn{2}{|c|}{ and } & & & \\
\hline 3. BIKET & 107.6 & 199.1 & 376.1 & 75.7 & $17.0^{* * * *}$ \\
\hline 4. ARMR & 47.4 & 38.8 & 153.9 & 44.6 & $22.0^{* * *}$ \\
\hline 5. ARMRT & 110.6 & 62.3 & 274.3 & 59.6 & $23.0^{* * * 4}$ \\
\hline 6. LEGR & 47.2 & 39.6 & 187.1 & 38.1 & $2.0^{* * *}$ \\
\hline 7. LEGRT & 135.7 & 86.7 & 300.0 & 0.0 & $30.0^{* * *}$ \\
\hline \multicolumn{6}{|l|}{ MOSOS-III } \\
\hline 1. TOTSCORE & 27.5 & 24.0 & 54.3 & 17.2 & $89.0^{* *}$ \\
\hline 2. TSCORE & 355.8 & 303.6 & 693.0 & 175.0 & $81.0^{4 * *}$ \\
\hline 3. DSCORE & 125.7 & 125.2 & 321.6 & 135.9 & $63.0^{* * *}$ \\
\hline
\end{tabular}

$* * p<.01, * * * p<.001$

Table 6.IX. Discriminative value of MOSOS-III and MOSOS-I: Comparison between male normals $(N=12)$ and male CLBP patients $(N=12)$ : Mann-Whitney $U$ test.

\begin{tabular}{|c|c|c|c|c|c|}
\hline & $\begin{array}{l}\text { CLBP } \\
M\end{array}$ & $\mathrm{SD}$ & $\begin{array}{l}\text { Normals } \\
\text { M }\end{array}$ & $\mathrm{SD}$ & $\mathrm{U}$ \\
\hline \multicolumn{6}{|l|}{ MOSOS-I } \\
\hline 3. BIKET & 270.8 & 154.7 & 418.3 & 5.8 & $32.5^{* *}$ \\
\hline 4. ARMR & 96.0 & 53.6 & 180.6 & 43.7 & $16.0^{*}$ \\
\hline 5. ARMRT & 190.1 & 93.0 & 292.5 & 26.0 & $26.0^{* *}$ \\
\hline 6. LEGR & 88.2 & 49.4 & 166.4 & 44.2 & $15.5^{* *}$ \\
\hline 7. LEGRT & 205.6 & 102.4 & 300.0 & 0.0 & $33.0^{* * * *}$ \\
\hline \multicolumn{6}{|l|}{ MOSOS-III } \\
\hline 1. TOTSCORE & 46.6 & 19.6 & 47.5 & 13.3 & 70.5 \\
\hline 2. TSCORE & 512.7 & 236.2 & 596.8 & 133.7 & 51.0 \\
\hline 3. DSCORE & 182.8 & 134.4 & 263.8 & 66.1 & 43.5 \\
\hline
\end{tabular}

$* * \mathrm{p}<.01$ 


\subsection{Construct validity of MOSOS}

If the MOSOS measures behavioral deficits, or residual healthy behaviors, of CLBP patients, it is hypothesized that the scores correlate negatively with measures of the overt-motoric dimension of pain. Pain behaviors are conceptualized to be incompatible with health behaviors such as physical performance. It is not expected that a significant correlation is to be found between the MOSOS scores and the verbal report of pain, as the latter is considered a measure of a different response system (in casu the verbalcognitive dimension) of chronic pain. One of the reasons why patients avoid physical activities might be that they fear that too much physical activity might damage their body, potentially causing more pain.

\subsubsection{Subjects}

The subjects described in the previous experiment also participated in this experiment.

\subsubsection{Procedure}

In this experiment the Kendall correlation coefficient Tau is calculated among the MOSOS scores and three other measures:

1. Self-report of pain: The Visual Analog Scale (VAS; Huskisson, 1983) is administered the same day the MOSOS is taken. The patient is asked to rate his current pain level on a $10 \mathrm{~cm}$ horizontal line on which two extremes are mentioned: no pain at all, and the worst pain you can imagine.

2. Overt pain behavior: A Dutch version of Richards et al's (1982) UAB Pain Behavior Scale (PGS; chapter 5) is used by a trained research assistant while subjects are engaged in the MOSOS tasks.

3. Fear of body injury: Scale BI of the Fear Survey Schedule (FSS; Wolpe \& Lang, 1977) is considered a measure of fear of tissue damage.

\subsubsection{Results}

Table 6.X displays the correlation coefficients between MOSOS scores and VAS, PGS, physical condition, and FSS-BI. In accordance with the hypothesis no correlations are found between MOSOS scores and VAS, underscoring the poor relationship between self-reported pain level and motoric behavior (also referred to as discordance). Also as expected, negative and significant correlations are found between MOSOS scores and PGS scores. For swimming performance, no relationship is found between pain behavior and time and distance scores. This is interesting as for the other time and 
Table 6.X. Criterium-related validity of MOSOS. Kendall Correlation Coefficient Tau between MOSOS scores, VAS, PGS, and FSS-BI.

\begin{tabular}{llll}
\hline & VAS & PGS & FSS-BI \\
\hline MOSOS-I & & & \\
1. WALKD & -0.15 & $-0.44^{* * *}$ & -0.14 \\
2. ARMR & -0.18 & $-0.31^{* *}$ & $-0.26^{*}$ \\
3. LEGR & -0.12 & $-0.27^{* *}$ & -0.09 \\
4. TSCORE & -0.16 & $-0.31^{* *}$ & $-0.23^{*}$ \\
MOSOS-II & & & \\
1. SITT & -0.17 & $-0.38^{* * *}$ & $-0.30^{* *}$ \\
2. STANDT & -0.15 & $-0.36^{* *}$ & -0.10 \\
3. REPLACN & -0.15 & $-0.42^{* * *}$ & -0.20 \\
4. REPLACT & 0.07 & $0.50^{* * *}$ & -0.05 \\
MOSOS-III & & & \\
1. TOTSCORE & & & $-0.21^{*}$ \\
2. TSCORE & -0.04 & $-0.30^{* *}$ & $-0.23^{*}$ \\
3. DSCORE & -0.03 & -0.13 & $-0.19^{*}$ \\
\hline
\end{tabular}

${ }^{*} \mathrm{p}<.05,{ }^{* *} \mathrm{p}<.01,{ }^{* * *} \mathrm{p}<.001$ (one-tailed)

distance scores a significant negative correlation with PGS is found. This finding suggests that although patients who exhibit extreme pain behaviors cannot walk, row, or ride a bicycle for a long time, they can swim long distances. Thus, the incompatibility between pain behaviors and physical performance does not apply for swimming tolerance. Of interest here is that sitting tolerance shows the strongest relationship with fear of tissue damage, as measured with FSS. This finding suggests that patients with a strong fear that they will harm their body, will avoid sitting activities more than other activities. One possible reason might be that these patients are more tense and restless, and will more often shift from sitting to standing position. Generally, the correlations found are in the expected direction. However, they are quite modest, with the highest tau 0.50 . This means that only a relatively small part of the variance in MOSOS scores can be explained by pain behaviors, and fear of tissue damage. Nevertheless, these findings appear to support the validity of the MOSOS as a measure for residual health behavior. 


\subsection{Discussion}

This chapter focused on the development of an assessment instrument of behavioral deficits in CLBP patients. Behavioral deficits were defined as behaviors that do not belong to the person's behavioral repertoire anymore, or less frequently as needed, and which appear in healthy and pain-free people. The assumption is that behavioral deficits of CLBP patients are the result of two alternative processes: $a$. Healthy behaviors are avoided because the person has learned that avoidance behaviors reduce suffering, or $b$. The pain problem has existed so long that a substantial skill deficit has been developed. It was decided to develop an instrument analogous to the BAT that is used in anxiety assessment. The most prominent feature of a BAT is that the person decides how far he or she goes in approaching the stimulus, and engaging in progressively more interactions with it. The MOSOS has the same feature and its instructions are based on the pain-rest contingency principle. The relative simple instruction limits variation in demand characteristics of the test. Anxiety assessment research has shown that high demands result in significantly more approach (Bernstein \& Nietzel, 1974). When taking the MOSOS the demands are held constant. Furthermore, the instrument is highly standardized, but easily applicable in a clinical setting.

Two parts of the MOSOS (MOSOS I: involving cyclic movements of the extremities, and MOSOS III: swimming) appear internally consistent, and normally distributed. This is not the case for MOSOS II, involving more static activities. In our sample older patients have lower scores than younger patients. Probably because the male patients are less disabled in our sample, male patients show more healthy behaviors than female patients.

The MOSOS appears to be psychometrically sound. Both reliabilty and validity checks underscore the value of the MOSOS as a potentially useful instrument. Moreover, the MOSOS appears to discriminate well between CLBP patients and healthy and pain-free controls.

A methodological weakness of this study concerns the relatively small sample sizes of the reliability and validity experiments, making replications of the findings warranted. On the other hand, non-parametrical statistical procedures have been used.

Further research should also examine the treatment utility (Hayes et al., 1988) of the MOSOS. If the instrument is specific and sensitive to treatment changes, it can be used as a treatment outcome measure in the evaluation of behavioral pain treatment programs. In that case, the instrument could provide adequate baseline levels of residual healthy behaviors that can be 
used to design an individually tailored behavioral and interdisciplinary treatment program.

A limitation of the test is that it only assesses avoidance of motoric activities. Other activities (e.g. socializing) are often avoided by pain patients as well (Philips \& Jahanshahi, 1986). On the other hand, the performance of many activities is only possible when motoric activities are not avoided. One disadvantage is that the MOSOS cannot differentiate between avoidance behavior and physical incapacities or disuse occurring as a consequence of longstanding avoidance behaviour. In both cases, however, treatment will occur quite similarly but with different effects. In the case of avoidance learning, a reactivation program acts an systematic exposure procedure, which eventually can be combined with relaxation. In the case of skill deficits, the reactivation program will proceed as an individual shaping program, aimed at restoring motoric skills. In clinical practice, the physical examination may help making this differentiation. In any case, observational data should be integrated with self-report and physiological data. 



\section{CHAPTER 7}

\section{The development of a pain cognition questionnaire*}

\subsection{Introduction}

In chronic pain, a limited number of studies have focused on cognitive assessment. Lefebvre (1981), basing his work on Beck's (1976) conceptualization of logical errors in the thinking of depressed patients, studied cognitive errors in both depressed and non-depressed low back pain patients using the General and Low Back Pain Cognitive Error Questionnaire $(C E Q)$. Cognitive errors are in this study defined as distorted or illogical inferences based on available information. Smith et al. (1986), using the $C E Q$, reported that cognitive distortion was consistently related to several aspects of disability in chronic pain. A limitation of the CEQ is that it exclusively focuses on "distorted' pain cognitions. The Ways of Coping Checklist (WCCL; Vitaliano et al,, 1985) is another questionnaire that, in part, examines attributions. However ${ }^{\prime}$ this instrument has not been designed for chronic pain patients.

None of the assessment instruments cited above focus specifically on the kind of things patients think of their pain, in terms of attributions and expectation. The current study is an attempt to develop an instrument that is able to do so and that might present a reliable measure for the verbalcognitive response system of chronic pain.

* This chapter is a slightly modified version of Vlaeyen, J.W.S., Geurts, S.M., KoleSnijders, A.M.J., Schuerman, J.A., Groenman, N.H., \& Van Eek, H. (1990). What do chronic pain patients think of their pain? Towards a pain cognition questionnaire. British Journal of Climical Psychology, 29, 4, 383-394. 


\subsubsection{Pilot study}

Based on the conceptual assumptions described above, as well as on the thoughts and clinical experiences of experts in chronic pain treatment, a provisional list of 81 pain cognition items was constructed. The operational definition of pain cognition we adhered to in this study was 'any verbalized evaluation related to both an evaluation of the pain and an evaluation of the extent to which the person is effective in dealing with the pain'.

The question: "To what degree are the following statements rellevant to you?" preceded the list of items. Following each item was a 5-point Likert rating scale from which the patients could answer that they were in total disagreement, relative disagreement, neutral, relative agreement, or total agreement.

The original list of items was presented to a group of nine chronic pain patients, three of whom had chronic back pain, another three of whom had spinal cord lesions, and still another three of whom had phantom limb pain. Because, in the future, the questionnaire was meant to be used for different diagnostic groups of pain patients, these three different diagnoses were included in this pilot study. After the administration of the provisional 81-item list, all nine patients were interviewed about formal aspects of the list (length and instructions) as well as about aspects regarding content (clarity and meaning of the items). Since the subjects experienced some difficulty with the instructions, the question was modified to read: "To what extent do you agree with the following statements?". The response categories remained unchanged. Four statements were eliminated because more than two subjects considered them to be irrelevant to their pain problem. The final list of 77 items was preceded by a visual analog scale (VAS) to measure the intensity of the pain experienced.

\subsubsection{Subjects}

The subjects were 188 chronic pain patients (53\% female and $47 \%$ male) with a mean age of 38.5 years $(S D=13.5)$ and 41.7 years $(S D=12.5)$, respectively. The mean ages of the two sexes were not significantly different $(t=1.66$, $\mathrm{p}=0.10)$. Of all the patients, $137(73 \%)$ were diagnosed as having chronic back pain, $19(10 \%)$ had rheumatoid arthritis, $15(8 \%)$ suffered from spondilitis

* Copies and norms of the Dutch version of the PCL can be obtained from Swets Test Services, PO Box 820,2160 SZ Lisse, The Netherlands. 
ankylopoetica, $7(4 \%)$ received the diagnosis fibromyalgia, and $10(5 \%)$ suffered chronic back pain with different diagnoses.

Most of the subjects were recruited from the Department of Rheumatology of the Lucas Foundation in Hoensbroek and from the Pain Clinic of the University Hospital in Maastricht. A smaller number of subjects came from the University Hospital of Leuven (Belgium). For these subjects, measures were taken during the clinical evaluation procedure. Through a local patient organization, a small group of patients was contacted by mail.

\subsection{Procedure}

All subjects received the 77 -item $P C L$. For all items, the frequency distribution was calculated. Only items with a quasi-normal distribution (skewness >-1 or $<1$ ) were subsequently subjected to a principal component factor analysis with varimax rotation. Items with a factor loading $<0.35$ on all factors were excluded. For those items having high factor loadings on more than one factor, correlations between the item and the factors were calculated. When the correlation coefficients differed by less than 0.05 , the item was excluded. When the difference was more than 0.05 , the item was assigned to the factor with which it correlated the highest.

\subsubsection{Results}

Twelve items were rejected because they were not sufficiently normally distributed. The remaining 65 items were subsequently subjected to a principal factor analysis with varimax rotation. With the decreased number of items the 3:1 ratio for subjects to items is reasonably approached. Using the scree test criterion for determining the number of factors to be extracted, five factors were obtained which cumulatively accounted for $39.9 \%$ of the total variance. Table 7.I shows the factor loadings for the items on each factor after varimax rotation. Eleven items were excluded because they lacked sufficient factor loading. Another twelve items showed high factor loadings on more than one factor. Four of these were excluded because correlation coefficients with the factors did not differ sufficiently. Fifty items finally remained and constitute the new PCL. Labels were assigned to the respective factors giving the most consideration to items with high loadings. Items of factor 1 appear to reflect the impact the pain has on the patients functioning (e.g. "I feel less and less capable of doing something"). Therefore, the label 'Pain impact' seemed appropriate. Factor 2 concerns self-statements focusing exclusively on the aversive aspect of the pain experience (e.g. "the word 'pain' frightens me"). Some items, with smaller 
loadings on this factor, also reflect a certain degree of helplessness (e.g. "I consider myself to be a hopeless case").

The label 'catastrophizing' is assigned to this factor. Factor 3 appears to represent the patient's expectancy that a given behavior will lead to certain outcomes (e.g. "relaxation exercises ease the pain"), and accordingly is labeled "outcome-efficacy". In contrast to factor 3 , factor 4 includes 4 items resembling a certain degree of passivity, and the expectancy that responding in a different way is futile and unnecessary (e.g. "I think the best thing to do is to wait and see what comes"). For this factor, the label 'acquiescence" was chosen. The items of factor 5 show the expectancy that change is possible, but that the change can only be produced by others (e.g. "Somebody must be able to help me"). The label 'reliance on health care' was therefore chosen for this factor. The major characteristics of the 5 factors, are summarized in Table 7.II.

Table 7.I. Item content, skewness, and factor loadings for Pain Cognition List items for each factor. (Items are translated from the Dutch.)

Item content

Skew- Factor-

ness laading

Factor 1: "Pain impact"

20. My attitude has not been influenced a great deal by my pain. $\quad-0.50 \quad-0.62$

41. I think that just continuing everything is the best for me. $\quad-0.65$

$-0.58$

65. Despite the pain, I look forward to the future with confidence. -0.34

$-0.56$

29. It think I have learned how to deal/cope with the pain.

$-0.56$

74. I feel less and less capable of doing something.

$-0.86$

0.54

14. I think I am actively engaged.

$-0.52$

$-0.53$

22. It looks like my pain comes to the front everytime.

$-0.55$

0.53

7 . I have diversion enough to keep the pain bearable.

0.04

$-0.52$

66. I have become physically weaker and weaker.

$-0.55$

0.50

21. I think I can manage for myself in daily life very well.

$-0.49$

0.50

69. My selfconfidence encourages me.

$-0.45-0.50$

43. Because of my pain I don't feel to be a fit person anymore.

$-0.62$

$-0.46$

54. If I go on like this, I think everything will be allright.

0.23

0.43

5. I feel handicapped by the pain.

$-0.10 \quad-0.43$

23. The pain does not keep me from enjoying my life.

6. Whether I feel pain or not, I always do what I had planned to do.

13. Because of the pain I appreciate life more than I used to. 
Factor 2: "Catastrophizing"

38. I often think back to the days when I had no pain.

1. My thoughts are concentrated on pain all the time.

11. I consider myself an unlucky person.

2. I think that fate hit me.

72. The word "pain" frightens me.

26. I often think: Why is this happening to me?

75. I think that, because of the pain ${ }_{r}$ I changed my view of life.

71. I feel powerless against the pain.

39. I think I live very tense.

47. I act very carefully to protect myself against extra pain.

61. I have become to feel psychologically weaker.

18. I consider myself to be a hopeless case.

10. When the pain is very strong I feel fury and anger grow in me. 0.11

64. I sometimes wonder how other people cope with severy pain. -0.94

3. Thinking too much about the pain makes it only worse.

Factor 3: "Outcome-efficacy"

52. I think tension increases the pain.

49. Relaxation exercises decrease the pain.

42. When I am busy doing something I can forget my pain considerably.

12. I think psychological factors have to do with my pain too.

46. I think I can influence my pain in a positive way.

32. I know a way to decrease my pain.

77. The weather influences my pain.

$-0.82$

Factor 4: "Acquiescence"

24. I think the best thing to do is to stay as calm as possible. $\quad-0.91$

58. Whatever I do, I cannot change my pain.

59. I think the best thing to do is to wait and see what comes.

$-0.36$

0.43

36. I think praying or hoping are the only things that I can do.

0.50

0.41

Factor 5: "Reliance on health care"

45. I think there is still hope.

68. Somebody must be able to help me.

15. I am convinced that there is a therapy to control my pain.

60 . I have the utmost confidence in medical science.

70. I think everybody should just leave me alone. 
Table 7.II. The Pain Cognition List factors $(N=188)$.

\begin{tabular}{llllll}
\hline $\begin{array}{l}\text { Factor } \\
\text { label }\end{array}$ & $\begin{array}{l}\text { Number of } \\
\text { items }\end{array}$ & $\begin{array}{l}\text { alpha } \\
\text { coeff. }\end{array}$ & $\begin{array}{l}\text { Scale } \\
\text { mean }\end{array}$ & $\begin{array}{l}\text { Scale } \\
\text { SD }\end{array}$ & $\begin{array}{c}\mathbf{r}_{\text {ab }} \\
(\mathrm{n}=34)\end{array}$ \\
\hline $\begin{array}{l}\text { 1. Pain impact } \\
\text { 2. Catastrophizing }\end{array}$ & 17 & 0.85 & 46.2 & 12.0 & $0.92^{* *}$ \\
$\begin{array}{l}\text { 3. Outcome-efficacy } \\
\text { 4. Acquiescence }\end{array}$ & 17 & 0.88 & 49.0 & 14.1 & $0.79^{* *}$ \\
$\begin{array}{l}\text { 5. Reliance on } \\
\text { health care }\end{array}$ & 4 & 0.70 & 22.6 & 5.7 & $0.81^{*}$ \\
\hline
\end{tabular}

$\mathrm{r}_{\mathrm{ab}}=$ Intra-rater Pearson correlation coefficient.

* ps.01, $p \leq, 001$ (one-tailed)

7.3. Examination of the relationship between factor scores and individual differences

Table 7.III shows the mean differences of factor scores for the male versus female group and for the chronic back pain (CBP) versus other diagnoses group. The t-test revealed no single significant difference for all PCL factors. It may be concluded that the PCL is stable across sex and diagnosis. Correlations between age and factor score are presented in Table 7.IV. Of interest, and rather unexpected, is the observation that age appears to be negatively correlated with factor 4 (acquiescence).

\subsection{Reliability}

In order to test the reliability of the PCL, internal consistency was examined. For the five factors, Cronbach's alpha was $0.85,0.88,0.70,0.63$, and 0.61 , respectively (Table 7.II). The lower values for PCL factors 3, 4 and 5 may be due to the limited number of items. Application of the Spearman-Brown correction for an extension to 17 items results in alpha values of $0.85,0.88$ and 0.84 respectively.

An additional study was set up to evaluate test-retest reliability. Thirty four chronic back pain patients who were admitted to the Lucas Foundation for Rehabilitation for an inpatient pain rehabilitation program were given the PCL during the baseline period on two occasions with a time interval of 2 weeks. It is assumed that pain cognitions remain stable during this period. As is displayed in Table 7.II, all intra-rater correlations $\left(r_{a b}\right)$ are statistically significant. The correlation coefficient for factor 'acquiescence', 
however, is quite low. This can be due to the small number of items belonging to this factor, limiting factor score variance. Another reason might be that, for some patients, this factor is sensitive for the expectancy that treatment will follow soon, and this is more the case at the second administration. Based upon these data, and the satisfactory internal consistency, it may be concluded that the PCL is sufficiently reliable.

Table 7.III. Relationship between PCL factor scores and individual differences including sex and diagnosis.

\begin{tabular}{|c|c|c|c|c|c|c|c|c|c|c|}
\hline \multirow[t]{3}{*}{ Fiactor } & \multicolumn{5}{|c|}{ Sex } & \multicolumn{5}{|c|}{ Diagnosis } \\
\hline & male & $(\mathrm{N}=85$ & female & $(N=94)$ & $t$ & $\mathrm{CBP}$ & $\mathrm{N}=137$ & Other & $(N=50)$ & t \\
\hline & $\mathrm{M}$ & $\mathrm{SD}$ & $\mathrm{M}$ & SD & & $\mathrm{M}$ & $\mathrm{SD}$ & $\mathrm{M}$ & SD & \\
\hline 1. & 46.2 & 11.3 & 46.7 & 12.6 & -0.28 & 46.8 & 12.8 & 46.0 & 10.6 & 0.45 \\
\hline 2. & 48.1 & 13.3 & 50.7 & 15.2 & -1.20 & 49.1 & 14.3 & 49.1 & 14.3 & 0.01 \\
\hline 3. & 22.4 & 5.4 & 22.8 & 5.9 & -0.49 & 22.1 & 5.6 & 23.4 & 6.0 & -1.36 \\
\hline 4. & 12.9 & 3.7 & 13.7 & 3.6 & -1.43 & 13.2 & 3.6 & 13.1 & 3.8 & 0.10 \\
\hline 5. & 18.3 & 4.1 & 18.3 & 3.8 & -0.04 & 18.3 & 4.1 & 18.5 & 3.2 & 0.32 \\
\hline
\end{tabular}

CBP: Chronic Back Pain, M: Mean, SD: Standard deviation.

${ }^{*} \mathrm{p} \leq .01,{ }^{* *} \mathrm{p} \leq .001$.

Table 7.IV. Correlations between age and PCL factor scores $(N=188)$.

\begin{tabular}{llllll}
\hline & & \multicolumn{5}{l}{ PCL Factor } \\
\hline Age & 1 & 2 & 3 & 4 & 5 \\
\hline & -0.07 & -0.06 & -0.06 & $-0.27^{*}$ & 0.09 \\
\hline
\end{tabular}

${ }^{*} \mathrm{p}<.001$ (one-tailed)

\subsection{Validity}

\subsubsection{Criterion-related validity}

An evaluation of the validity was conducted on all of the factors by examining the relationship between the PCL factors and theoretically relevant psychological variables as criterion variables. In the absence of reliable and valid Dutch attribution and expectation questionnaires, it was decided to 
focus on the relationship with depression and locus of control. From a cognitive-behavioral perspective, both considered two important constructs. Two measures of depression are chosen: The Depression scale of the Minnesota Multiphasic Personality Inventory (MMPI D; Hathaway \& McKinley, 1967) and the 'Depressive Reaction' scale of the Utrecht Coping List (UCL). The UCL (Schreurs et al., 1984) is a reliable Dutch coping questionnaire. A Dutch version of the Multidimensional Health Locus of Control scale (MHLC; Wallston et al. 1978) is used (Halfens, 1985). The MHLC assesses patients' health-related locus of control with the factors 'internal", "chance' and 'powerful others'.

Hypotheses regarding the expected correlations are formulated, based upon findings in the literature. In table 7.V the hypothesized relationship among the criterion measures and the PCL factors is shown by the symbols ' + ' and ' - '. A ' + ' indicates that a positive correlation is expected, " - " a negative correlation. The absence of a symbol corresponds with the expected absence of a significant correlation. Finally the effect of defensiveness will be examined, and the correlations with the reported pain intensity.

This experiment included data from 43 chronic back pain patients who were admitted to a multidisciplinary pain management program at the Rehabilitation Center in Hoensbroek (The Netherlands). The patients administered the PCL on 2 occasions. At the first occasion they also completed the MMPI and the UCL. At the second occasion they also completed the MHLC.

Table 7.V. Criterion Validity: Pearson correlation coefficients among PCL factors, MMPI $D^{1}$, UCL depressive reaction ${ }^{1}$, MHLC factors ${ }^{1}, M M P I K-$ $F^{1}$, and $V A S^{2}$.

\begin{tabular}{lccccc}
\hline & \multicolumn{5}{c}{ PCL Factor } \\
\cline { 2 - 6 } & 1 & 2 & 3 & 4 & 5 \\
\hline MMPI D & $+0.40^{*}$ & $+0.42^{*}$ & 0.02 & 0.15 & --0.23 \\
UCL Depressive reaction & $+0.51^{* *}$ & $+0.43^{*}$ & 0.17 & 0.10 & $--0.51^{* *}$ \\
Locus of control & & & & & \\
MHLC-Internal & 0.29 & 0.26 & +0.38 & $-0.56^{* *}$ & -0.15 \\
MHLC-Powerful other & -0.14 & 0.37 & -0.08 & 0.38 & --0.09 \\
MHLC-Chance & 0.17 & $+0.72^{* *}$ & -0.00 & +0.33 & -0.05 \\
MMPI K-F & -0.28 & $-0.46^{* *}$ & -0.15 & -0.03 & $0.36^{*}$ \\
VAS & $0.28^{* *}$ & $0.24^{* *}$ & -0.12 & $0.25^{* *}$ & 0.06 \\
\hline
\end{tabular}

${ }^{1} \mathrm{~N}=43,{ }^{2} \mathrm{~N}=188 ; * \mathrm{p} \leq .01, * * \mathrm{p} \leq .001$ (one-tailed). 


\section{Depression}

Consistent with the cognitive-behavioral perspective, depressed chronic back pain patients are more likely to be characterized by certain distorted attributions (such as catastrophizing thoughts) than non-depressed controls (Lefebvre, 1981; Smith et al., 1986). Therefore it is hypothesized that the measures of depression correlate positively with PCL factor 2. Also for PCL factor 1, representing the negative impact the pain has on the patient's life, possibly leading to a deprivation of reinforcing events because of pain, a positive correlation with depression is expected. In contrast, factor 5 , reflecting a positive future perspective, is hypothesized to correlate negatively with the depression measures. For the other factors, no significant correlations are expected.

The results shown in table 7.V confirm the hypotheses, except the lack of a significant correlation between PCL factor 5 and MMPI D.

\section{Locus of control}

Attributions can be characterized along the dimension of locus (whether the cause is located inside or outside the person). If some of the PCL factors are measuring attributions, a relationship with locus of control is likely to occur.

MHLC 'internal' is expected to correlate positively with PCL factor 3 and negatively with $\mathrm{PCL}$ factor 4 and 5 . For PCL-factor 2 and 4 , a positive correlation is expected with MHLC 'chance'. Finally, PCL factor 5 is hypothesized to correlate positively with MHLC 'powerful others'.

The majority of the correlations (Table 7.V) appear to be in the expected direction, two of which reach a significant level. Only the correlation between MHLC 'powerful others' and PCL factor 5 cannot be confirmed, making further validation of this factor necessary.

\section{Defensiveness and reported pain intensity}

Another point of interest is the extend to which the PCL scores are influenced by the patient's test-taking attitude. The MMPI K-F raw score (based on the MMPI validity scales $K$ and $F$ ) is considered a measure of defensive testtaking attitude. Table 7.V shows a significant negative correlation between MMPI K-F and PCL factor 2, and a positive correlation between MMPI K-F and PCL factor 5. These data suggest that 'catastrophizing' might be underreported, and 'reliance on health care' be overreported by patients who approach the PCL in a defensive way.

The Visual Analog Scale (VAS) for pain intensity is a frequently used measure for the amount of pain experienced (Huskisson, 1982). Table 7.V presents positive correlations beween the VAS and PCL factors 1,2 , and 4 , 
respectively, suggesting that the remaining factors, 'outcome-efficacy' and 'reliance on health care', may occur regardless of pain intensity.

\subsubsection{Predictive validity}

Predictive validity is at issue when an instrument is developed to estimate some form of behavior that is external to the measuring instrument itself. In the case of the PCL, the question can be raised whether the PCL distinguishes patients whose pain problem is mainly controlled by cognitive factors from patients whose pain problem is mainly controlled by environmental contingencies. In order to answer this question, a third study involving 19 chronic back pain patients was set up. The patients were psychologically screened before entrance into a pain management program. Although clinicians' judgements can be unreliable, no other criterion measure was available.

Based on the interview data, two clinical psychologists independently placed the patients in two categories: a cognitive group and an operant group. The cognitive group was defined using the criterion that at least two of the following characteristics should be present: worrying, a rigid attitude, high achievement motivation, little avoidance of activity, and phobic or compulsive behavior. For the operant group, the following criterion was used: use of the word "unpleasant" to describe a return to work, increased attention because of pain complaints, and avoidance of activity. Seventeen of the 19 patients were put into the same category by the two judges $(89.5 \%$ agreement). Six patients were assigned to the operant group, while 11 patients were assigned to the cognitive group. For these 17 patients, the relationship between patient group and PCL scores is examined using the Mann Whitney U-test. The results suggest significant relations between the patient group (cognitive vs. operant) and PCL factor 1 ( $\mathrm{p}<.001$ ), factor 2 $(\mathrm{p}<.01)$, and factor 5 ( $\mathrm{p}<.05)$. The cognitive group scored significantly higher on PCL factors 1 and 2, while the operant group scored significantly higher on PCL factor 5.

Based on the available data, it may be concluded that the validity of the PCL appears encouraging.

\subsection{Discussion}

The purpose of the present study was assessing the kind of things chronic pain patients say to themselves in relation to the pain suffered, and subsequently to examine the reliability and validity of an assessment instrument presenting a measure for the verbal-cognitive response system of pain. 
After an item analysis and item reduction, a 50-item Pain Cognition List emerged, measuring five factors which are labeled as: pain impact, catastrophizing, outcome-efficacy, acquiescence, and reliance on health care. The PCL was developed using a Dutch back pain patient population and has proven to be stable across sex and back pain diagnosis. The PCL has also proven to be reliable and reasonably valid. Although causal inferences are precluded in any correlational study, the validity data seem to support the labels assigned to the five PCL factors.

This study assessed patients' self-statements about the pain and the extend to which they are effective in dealing with the pain. This not necessarily maps itself out onto a conceptual framework. The question can be raised whether the results underscore the importance of causal attributions and efficacy expectations, both important sets of cognitive variables postulated from the cognitive-behavioral perspective. Further research will be necessary to test the relationships among the PCL factors and attributions and expectations. A problem, however, is that the use of an endorsement method always holds the implicit acceptance of the notion that the cognitions under examination are within the conscious awareness of the subject. This motion has recently been challenged (Van den Bergh \& Eelen, 1984).

Some methodological weaknesses make the conclusions of this study tentative. First, there is some heterogenity with respect to diagnosis and recruitement of the original sample on which the factor analysis is carried out. It is not clear, for example, whether differential recruitement (evaluations via mail vs. clinical evaluations) and the different diagnoses included in the study affected the results. On the other hand, the PCL appeared stable across sex, and also across diagnosis when the CBP-group is compared with the other diagnoses-group. Second, sample sizes for the reliability and validity experiments are relatively small, making replications of the findings warranted.

It has already been suggested that the three-systems model of emotions can successfully be applied to chronic pain. In spite of its weaknesses, the PCL might contribute to the assessment of cognitive factors. The predictive validity data seem to support this.

Pain patients who are classified as having pain which is substantially controlled by cognitive factors reportedly experience more negative impact of the pain on their lives, have more catastrophizing thoughts, and rely less on the health care system than pain patients whose pain problem is considered to be controlled by mainly operant factors.

Another question of interest concerns to what extent the cognitions, as measured by the PCL, change as a consequence of therapeutic interventions. The PCL might serve as a useful tool in evaluating the effectiveness of pain 
treatment. More research, as suggested by Hayes et all. (1987), is needed, however, before the treatment utility of the PCL can be fully established. 


\section{PART III:}

\section{INTERDISCIPLINARY TREATMENT OF CLBP IN REHABILITATION}

Recent studies have demonstrated the usefulness of different behavioral treatments for chronic back pain problems. Following the three-systems model of chronic pain, these treatments generally reflect one of three approaches, depending on the response system that is chosen as the therapeutic point of application. One approach, operant conditioning, aims to decrease pain behaviors and increase well behaviors by modifying social and environmental contingencies. The second, cognitive treatment, aims to modify the patients distorted pain cognitions and emphasizes patient acquisition of cognitive skills for coping with pain. The third, respondent approach, aims to decrease inappropriate tension and pain levels elicited by personally relevant stimuli. This approach, concerning the application of systematic desensitization and exposure, has received little attention in recent years. In spite of its relevance, the respondent approach falls beyond the scope of this study, and will be excluded from further discussion.

Extensive reviews on the effectiveness of the operant and cognitive approaches include those reported by Turner \& Chapman (1982), Linton (1986), and Cohen et al. (1989). Turner \& Chapman (1982) reviewed the literature through 1980 . Their conclusion was that operant treatment programs appear to increase physical activity levels and decrease medication use while the patients are in the controlled hospital environment. Few studies included follow-up assessments. In most studies, a careful selection 
took place with numerous criteria, possibly threatening external validity. The cognitive-behavioral treatment programs had the potential for relieving reported pain in a variety of chronic pain syndromes. However, among other shortcomings noted was the lack of objective outcome measures, such as observer ratings of pain behaviors and health care utilization.

Linton (1986), who examined studies which appeared between 1981 and 1985 , is clearly positive about the operant treatment. He concluded that the additional studies provide considerable support for its usefulness. Compared to earlier studies, the research qualities of the studies increased. Besides increase in activity levels, and decrease in medication use , improved mood is also noted. In his review, no use of 'pure' cognitive strategies was found. Linton concludes that there is no empirical evidence that cognitive aspects added value to other therapeutic ingredients of multimodal therapies.

Cohen et al. (1989) reviewed the more recent literature up to 1988. These recent studies have improved methodology: dependent variables included blind ratings of outcome by experts, objective measures of change, and measures of health care utilization or medications. It is remarkable, however, that 1. few experiments evaluate unimodal and pain-related cognitive therapies, and 2. few experiments have attempted to determine what aspects of cognitive-behavioral therapy are effective. After 1985, only two studies have evaluated the operant approach (Kerns et al., 1986; Turner \& Clancy, 1988). They both showed that the operant treatment can successfully be employed in out-patient settings. Turner \& Clancy (1986), who compared an operant-behavioral (OB) with a cognitive-behavioral (CB) approach, found that the operant approach showed better results post-treatment. Generally, however, the $O B$ patients showed a leveling off in improvement at 6-and 12-month follow-ups, whereas the CB patients generally continued to improve over the 12 months following treatment. At 12-month followup, patients from both treatments showed no significant differences.

In the Dutch health care system, controlled clinical research examining the efficacy of inpatient pain management programs, based on the above mentioned trichotomy has not been carried out yet. Some existing case studies, however, are promising (Cosyns \& Vlaeyen, 1984; Groenman et al., 1986; Vlaeyen et al., 1987).

In this section, the effectiveness of two interdisciplinary behavioral rehabilitation treatments is examined. Chapter 8 presents a case study in which an individually tailored operant treatment program is applied to a CLBP patient with sitting and standing tolerance problems. Chapters 9 and 10 examine the effectiveness of an operant and an operant-cognitive treatment implemented in a Dutch rehabilitation center. Chapter 9 focuses on the statistically significant changes of dependent variables that are related 
to the three-systems model of chronic pain. In chapter 10 , clinical significance of change during treatment, post-treatment levels, and follow-up levels is evaluated. Chapter 11 focuses on the broad-spectrum analysis. Subjects with similar problem areas other than pain are clustered in classes, and the relationship among these classes and treatment outcome is highlighted. Chapter 12 examines the desynchronous relation among the overtmotoric and the verbal-cognitive response systems during both experimental treatments. Finally, chapter 13 provides a methodological justification of the investigations described in chapters 9 to 11 . 

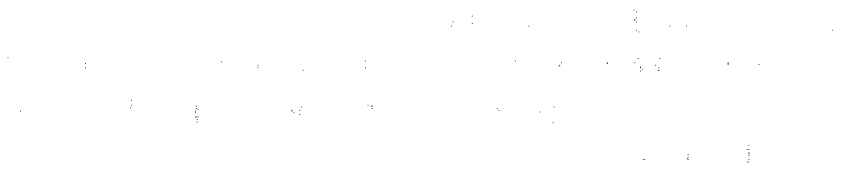

?.

. 


\section{CHAPTER 8}

\section{A behavioral treatment for sitting and standing intolerance in a patient with chronic low back pain*}

\subsection{Introduction}

Low sitting and standing tolerance, resulting in frequent shifting from one buttock to the other and from the standing to the sitting position, is a frequently observed pain behavior in chronic back pain patients. In the acute phase, these problems may result from a structural or mechanical defect. In the chronic phase, at least three factors can be identified that may contribute to the maintenance of these problems (Fordyce, 1976), namely:

1. It may be that the inability to sit has persisted so long that a substantial skill deficit for normal sitting tolerance has developed. The patient may no longer know how to sit properly.

2. This pain behavior may reflect avoidance learning. Intolerance may have begun in response to the acute pain situation, as a way to ease or reduce the pain. Distorted sitting may persist, in part because the patient has ceased testing whether the pain will return if these "protecting' or avoidance behaviors are not carried out anymore. In that case, they exist in anticipation of pain, rather than in response to it.

3. The protective pain behaviors may elicit so much attention from the environment that the patient is not encouraged to emit well behaviors.

Generally, it can be concluded that, in chronic benign back pain, the inability to walk or to sit properly is a learned behavior which may lead to

* This chapter is a slightly modified version of Vlaeyen, J.W.S., Groenman, N.H , Thomassen, J. Schuerman, J.A., Van Eek, H., Snijders, A.M.J, \& Van Houten, I. (1989). A behavioral treatment for sitting and standing intolerance in a patient with chronic low back pain. The Clinical Journal of Pain, 5, 233-237. 
muscle disuse, causing more pain. In this manner, a vicious circle leads to a perpetual pain problem in the chronic phase.

One of the strategies that can be used to stop the above mentioned vicious circle is based on relearning well behaviors. This relearning process can be enhanced by a gradual introduction of normal behaviors into the patients repertoire. This process is referred to as "shaping", which is defined as "developing a new behavior by reinforcing successive approximations toward the terminal response" (Kazdin, 1980). Although some studies have reported on the efficacy of shaping procedures in rehabilitation (e.g. Fordyce et al,, 1968; Brockway \& Steger, 1981; Doleys et al., 1982) a shaping program for low sitting and standing tolerance in chronic low back pain has not been described yet. The present case study expands the number of various shaping procedures and documents the effect of a specific, individually tailored shaping program.

\subsection{Subject}

The patient is a 50-year-old, unmarried, Caucasian female shopkeeper with a 2-year history of constant burning chronic low back pain. The pain started after a laminectomy (L2-3-4) for removal of an intradural tumor. A postoperative examination revealed an ependymome without any sign of malignancy. Although a subsequent extensive neurological and orthopedic examination revealed no significant anomalies, the frequency of pain episodes and functional restrictions increased. The pain reportedly was worse when sitting, during which the patient had the feeling her whole back would 'collapse'. Previous medical interventions included appendectomy, an eye operation for strabismus, intra-abdominal adhesiolysis, and treatment of subacute thyreoiditis, all of which proceeded without complications.

At the time of her admission to the rehabilitation center, the patient was unable to sit for more than 1 minute or to stand still for more than 30 seconds. At home she would spend most of the time lying in her bed, which had been placed in her office, next to the store. She would do some of her office work in the lying position. Many of her regular customers would visit her regularly when entering the store. During the initial interview, she continuously shifted to a standing position or walked back and forth across the room, but spent most of the time lying on the floor.

After a physical and a psychological screening procedure, an inpatient behavioral treatment, developed for this case of sitting and standing intolerance, was started. 
All clinical tests were explained to the patient before procedures were done. Testing included a standard assessment battery for chronic pain patients, and was repeated with a 2-week interval: three times before treatment, three times during treatment, and three times following treatment. Finally, assessment was repeated at a 6-month follow-up. This battery consisted of functional assessment, observational measures and a self-report measure.

Functional assessment included the assessment of sitting and standing tolerance. The standard instruction was based on a pain-rest contingency: the patient was asked to sit and stand as long as possible, i.e., until pain prevented her from continuing.

The extent to which this patient exhibited pain behavior was quantified by means of the UAB Pain Behavior Scale (see chapter 5). With this scale, a research assistant rates the severity or frequency of 10 pain behaviors on a 3 point scale. The 10 behaviors include verbal pain complaints, non-verbal vocal pain complaints, downtime, facial grimaces, standing posture, mobility, body language, equipment use, stationary movement and medication use. Richards et al. (1982) consider the scale to be reliable and to be sensitive to treatment-related changes. It is also negatively correlated with well behaviors such as sitting and standing.

As a self-report measure of the verbal-cognitive dimension of pain, the Visual Analog Scale (VAS; Huskisson, 1983) was chosen to quantify the intensity of the pain experienced.

\subsection{Behavioral program}

Based on the available data, it was decided that this patient was a good candidate for an 8-week, interdisciplinary, behavioral pain treatment program. In this case, pain behavior consisted of the inability to stand or to sit still in anticipation of increasing pain. The patient agreed that the ability to sit still for 15 minutes and to stand still for 25 minutes were realistic therapy goals. In order to achieve these goals, a behavioral shaping program, tailored to the individual needs of this patient, was developed by the psychologist and the physiotherapist (Table 8.I.). This program consisted of 21 hierarchically ordered, consecutive steps, ranging from much mobility to immobility and gradually progressing toward increasing weight on the buttocks. The program was developed following the principles reported by Fordyce (1976). After a 2-week baseline period, the shaping program was initiated by the physiotherapist with the following instructions: each 30-minute 
therapy session (to be held twice a day) is to start with the first item. When this step is mastered (i.e., carried out properly for 2 minutes), the next is to be added to it, and so forth, until therapy time is over. Ample social encouragement and reinforcement is provided by all therapists any time a sub-goal is reached. Before starting on the next item, however, all previous items must be carried out for 10 seconds. This allows for frequent practice of the new behavior. Moreover, at each point in time during treatment, the level reached is to be practiced further during occupational therapy and on the ward. Upon arrival at step 16, standing in a normal position should be possible. This newly learned behavior is then to be practiced during occupational therapy, where the time spent in the standing position is gradually increased. The physiotherapist continues with the remaining steps. When step 21 is reached, normal sitting is possible. Generalization is added by replacing the adjustable stool with a normal chair and by increasing the amount of time spent sitting. Furthermore, the patient's sister is invited several times during the treatment to discuss how she can positively reinforce the patient"s new well behavior.

\subsection{Statistical analysis}

Although statistical tests for single-case research have been applied with increased frequency over the last decade, their use still remains the exception rather than the rule (Kazdin, 1982). In the single case, the assumption of independence of error terms, which is necessary for the application of analysis of variance (ANOVA), is said to be violated. Shine and Bower (1971), however, support the use of the one-way ANOVA with repeated measurements in single-case designs. They consider a single person a response generator, the responses being statistically independent and normally distributed. Shine and Bower (1971) suggest the mean square successive difference between measures as the appropriate error term. In this study, we have chosen to use ANOVA according to these authors, comparing the pre-treatment, treatment, and post-treatment assessment phases.

\subsection{Results}

Figure 8.1. shows the evolution during treatment. In 12 physiotherapy sessions ( 30 minutes each), step 16 is reached. From this moment on standing time is increased in occupational therapy (Figure 8.2.). Four sessions later, step 21 is reached. Stepwise increments for sitting time are now introduced 
1. Walking, arms swinging at sides.

2. Marching in place, arms swinging at sides.

3. Between parallel bars, marching in place, while holding on to the bars, with heels and toes coming off the floor.

4. Marching in place, only heels come off the floor

5. Marching in place, unlocking and locking knees alternately, with heels and tores not coming off the floor.

6. Same as step 5, butt arms lightly on bars.

7. From balanced standing posture, hands at sides, feet always flat, unlocking and locking knees alternately.

8. Same as step 7, but hands on bars.

9. Weight shifting foreward and backward in place, knees straight, heels and toes off floor, hands swinging at sides.

10. Same as step 9, but hands on bars.

11. Weight shifting foreward and backward with stretched knees, with only heels coming off the floor; arms may swing at sides.

12. Same as step 11 , but hands on bars.

13. Weight shifting in place from leg to leg, knees straight, feet flat on floor, hands lightly on bars.

14. Standing on scales, between parallel bars, for 2 minutes with good posture while holding on to bars and with weight equally distributed between lower extremities. Arms may swing at sides.

15. Same as step 14, but with hands on bars.

16. Same as step 15 , but with arms straight at sides.

17. Standing on scales, sitting on a high stool, feet bearing $80 \%$ of the total body weight.

18. Same as step 17 , but stool is lowered, so that feet bear $60 \%$ of the total body weight.

19. Same as step 18 , but stool is lowered, so that feet bear $40 \%$ of the total body weight.

20. Same as step 19 , but stool is lowered, so that feet bear $20 \%$ of the total body weight.

21. Same as step 20, but stool is lowered, so that feet bear only the weight of both legs.

in occupational therapy. Figure 8.3 shows the overall course of functional assessment including sitting and standing tolerance time $(\mathrm{F}=9.11, \mathrm{p}=0.03$; $\mathrm{F}=25.21, \mathrm{p}=0.007$, respectively). Therapy results are fairly well maintained up to 6 months after termination of the shaping program. The observed pain behavior decreases significantly ( $F=31.677, p=0.005$; Figure 8.4), suggesting that the program resulted in an overall decrease in pain behavior. The pain intensity measured with the VAS, however, remains unchanged $(\mathrm{F}=2.14, \mathrm{p}=0.233)$. 


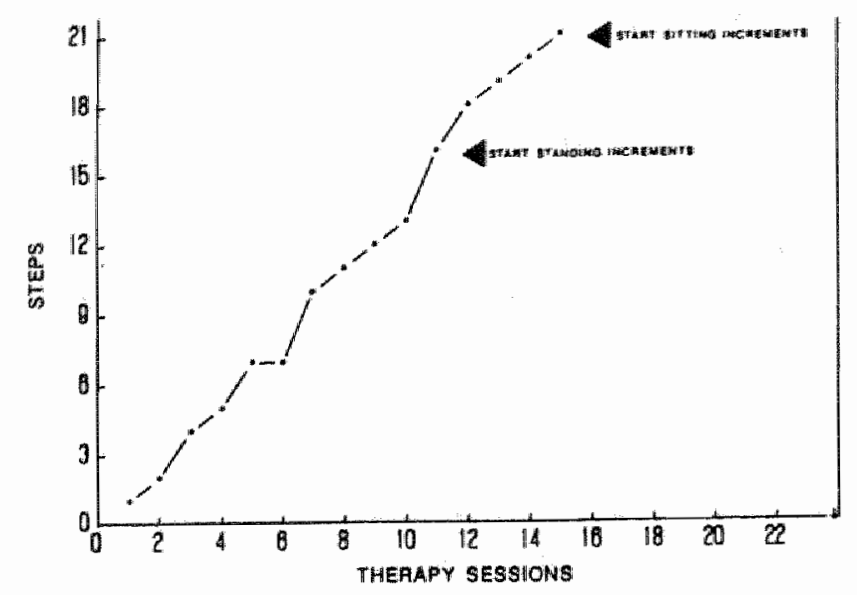

Figure 8.1. The evolution of the shaping program during treatment.

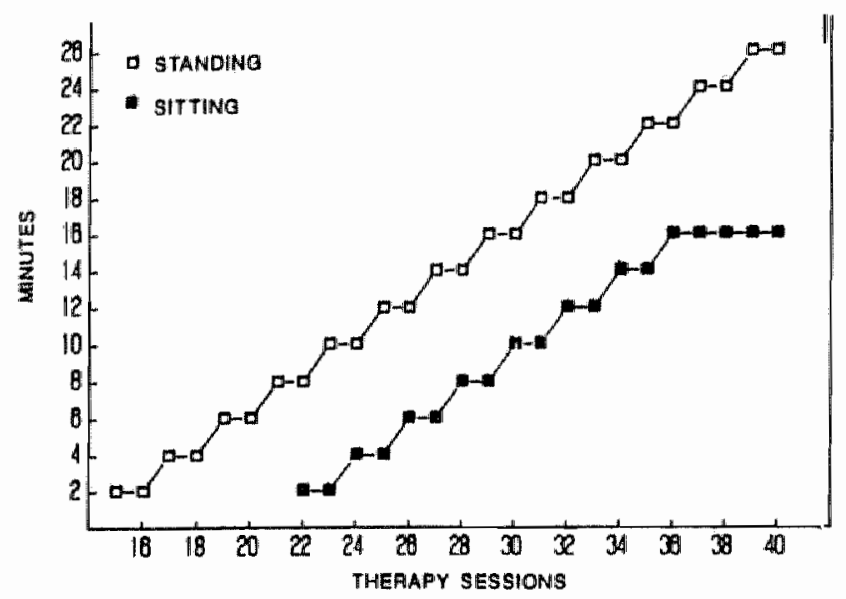

Figure 8.2. Standing and sitting time increments after termination of the shaping program.

\subsection{Discussion}

Individually tailored shaping procedures provide an effective way of dealing with various pain behaviors in chronic pain. Learned avoidance behaviors, which may be seriously disabling, can be extinguished quite rapidly, and well behavior can gradually be relearned. This study has underscored the importance of this relatively simple and cost-effective procedure in this 


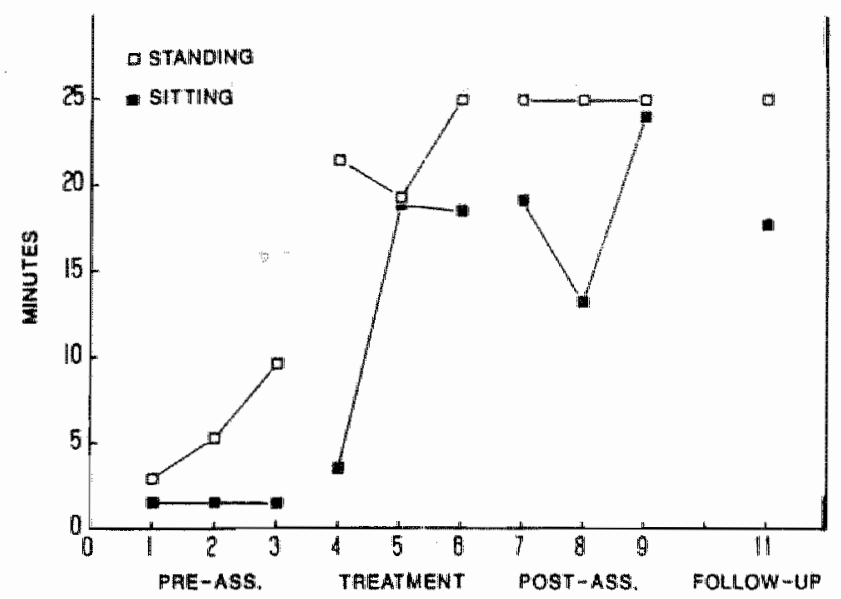

Figure 8.3. Standing and sitting tolerance time across baseline, treatment, post-treatment, and follow-up conditions.

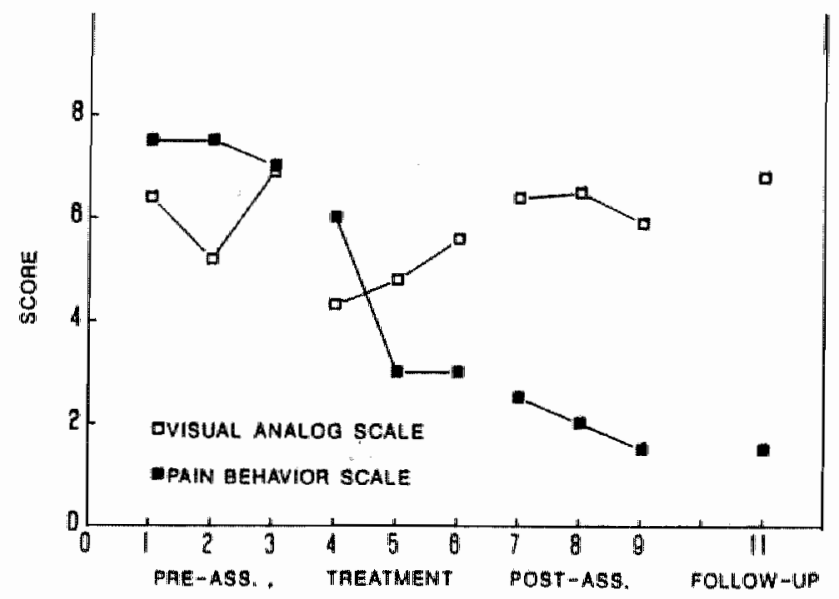

Figure 8.4. Observed pain behavior and self-reported pain intensity scores across baseline, treatment, post-treatment and follow-up conditions.

case of chronic low back pain. The patient not only showed rapid increases in sitting and standing tolerance time, but overall pain behavior also diminished significantly. Although functional and behavioral measures changed significantly, the self-report measure focusing on the pain experience did not. This finding supports the hypothesis of a discordant and desynchronous relationship between the cognitive and the motoric response system of chronic pain. It seems likely that treatment programs could be 
developed that are aimed at one response system at a time. Although the primary therapy goals have been achieved, a cognitive program focused on relabeling of the pain experience, would be a logical additional treatment goal in this case. 


\section{CHAPTER 9}

\section{Effectiveness of an inpatient operant and an operant-cognitive treatment for chronic low back pain I: statistical significance}

\subsection{Introduction}

This study describes a controlled evaluation of an operant treatment (OP) and an operant-cognitive treatment $(\mathrm{OC})$. The development of both treatment modalities (provided in Appendices A and B) has been guided by three criteria. First, an attempt was made to disassemble existing multimodal treatment programs into modalities that fit with the conceptual model outlined in chapters 1 and 2. An 'as pure as possible' operant and cognitive treatment was aimed at. Second, while chronic pain presents a multidimensional problem, the scientific rule of parsimony dictates that treatment should be done with the fewest number of effective ingredients. Of course, agreements with insurance carriers make the inclusion of some ingredients necessary. Third, the programs were to be fit into the Dutch health care system. In the existing Dutch rehabilitation centers, implementation of a cognitive treatment only, without participation of rehabilitation disciplines other than psychology, is not possible. Therefore, a "constructive approach" (Kazdin, 1982) was chosen according to which the cognitive treatment was added to the operant treatment as a second experimental condition.

\subsection{Method}

Figure 9.1. shows the repeated measurements design, consisting of 4 measurements before treatment (PRE), 3 measurements during treatment (TREATMENT), and 3 measurements after treatment (POST), with a time interval of 2 weeks respectively. In addition, a 6-month follow-up (FU1) 


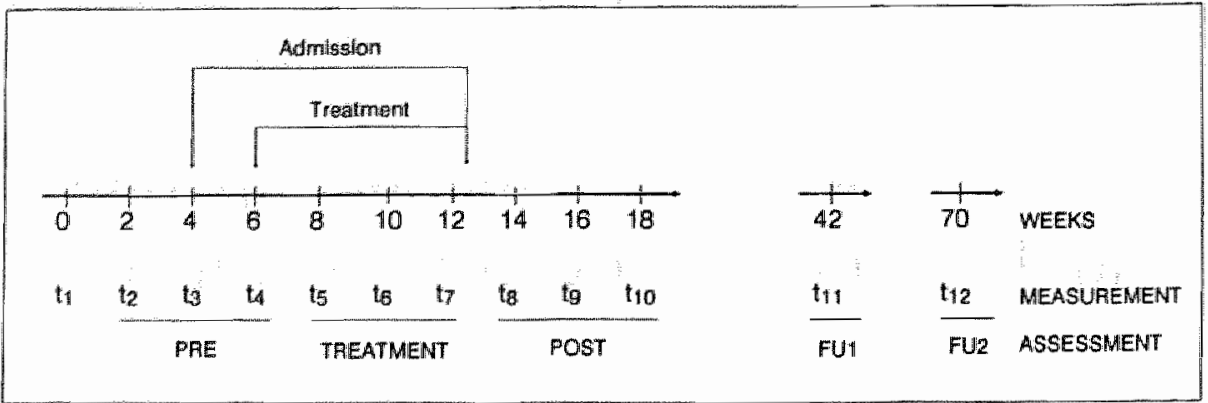

Figure 9.1.: Design

and a 12-month follow-up (FU2) assessment was carried out for the OPgroup, each consisting of only one measurement. For the OC-group, only FU1 was completed. This design allows the impact of treatment to be examined across time by using the subjects as their own control. Nevertheless, a waiting list control group (WLC) was included in the study. For practical reasons, the WLC only received 6 measurement points, also spaced at 2 week intervals. In a clinical setting, a placebo control group is extremely difficult to realize.

\subsubsection{Subjects}

Subjects were chronic low back pain patients referred to the study by the Pain Clinic of the University Hospital of Maastricht, The Netherlands. Criteria for acceptance into the study included:

a. persistent low back pain for at least 6 months,

b. age between 20 and 60 years,

c. a discrepancy between the pain complaints and the physical findings.

d. spouses, or other significant relatives/friends must be willing to participate in a special group instruction program.

Exclusion criteria comprised:

a. specific medical disorders and diseases selected to exclude subjects in immediate need of medical treatment (such as infectious diseases, fractures) as well as those who were unable to participate in a physical reactivation program (e.g. cardiovascular disease),

b. substance abuse,

c. involvement in any litigation concerning social disability income.

d. psychosis or organic brain damage.

e. analphabetism.

f. pregnancy in women. 
The subjects were not randomly assigned to the experimental and control conditions before the start of the study. The assignment was based on a time criterion. Patients who were referred in the first 18 months of the study, during which a cognitive treatment was not available yet, received the operant treatment (OP). Subjects who arrived in the subsequent 18 months received the operant treatment in combination with the cognitive treatment (OC). The waiting list control group (WLC) consisted of subjects who were chosen from an existing waiting list of the Rehabilitation Center of the Lucas Foundation for Rehabilitation, Hoensbroek, The Netherlands.

\subsubsection{Drop-outs}

Of the OP group 21 patients were selected, of which 2 male patients dropped out from the study, just before treatment. 1 and 3 patients did not return for FU1 and FU2 respectively. For the OC group 23 patients were selected. Five patients dropped out from the study: 2 male patients failed to come back for the post-treatment assessment, 1 male patient did not agree with the treatment goals, the fourth patient became pregnant just before the start of treatment, and the fifth (female) patient received an indication for acute care. At FU1, 3 patients did not show up. In the WLC group, only one (female) patient dropped out because she started therapy at another hospital.

\subsubsection{Biographical data}

The total group consisted of 38 female and 12 male chronic low back pain patients. Tables 9.I and 9.II show relevant biographical data for the respective groups, including sex, age, duration of pain complaints, number of back surgeries prior to admission, unsuccessful use of TENS and nerve blocks prior to admission, civil status, job status, education level, and received benefits. Patients in the three conditions did not differ on most of these biographical variables as tested by univariate ANOVA's and $\mathrm{CHI}^{2}$ analyses. Differences only exist for the variables 'received benefits' and prior unsuccessful use of TENS and nerve blocks. These differences may be due to the different referral source of the WLC group. This group was referred by another hospital, where TENS and nerve blocks are not commonly used. In the WLC group, also less patients received $80-100 \%$ disability income. ${ }^{*}$ Of interest is that the total group consists of patients with longstanding pain problems, the average time since the onset of back pain being 11.66 years $(\mathrm{SD}=8.82$, range $=1-37)$.

* In the Dutch social security system $80-100 \%$ disability corresponds with the maximum amount of benefit, which is $72 \%$ of the most recent income. 
Table 9.I. Biographical data I. CHI ${ }^{2}$ test for independent samples.

$$
\text { OP group OC group WLC group } \mathrm{CH}^{2} \quad \mathrm{p}
$$

\begin{tabular}{|c|c|c|c|c|c|}
\hline \multicolumn{6}{|l|}{ SEX } \\
\hline Male & 4 & 4 & 4 & & \\
\hline Female & 15 & 14 & 9 & 0.45 & .80 \\
\hline \multicolumn{6}{|l|}{ CIVIL STATUS } \\
\hline Married & 14 & 12 & 9 & & \\
\hline Widow & 0 & 0 & 1 & & \\
\hline \multicolumn{6}{|l|}{ Unmarried: } \\
\hline with partner & 3 & 0 & 0 & & \\
\hline without partner & 0 & 2 & 3 & & \\
\hline \multicolumn{6}{|l|}{ Divorced: } \\
\hline with partner & 0 & 4 & 0 & & \\
\hline without partner & 2 & 0 & 0 & 17.78 & $.05<p<10$ \\
\hline
\end{tabular}

LEVEL OF EDUCATION (ARBI ${ }^{*}$ classification)

\begin{tabular}{llllll} 
I & 4 & 4 & 4 & & \\
II & 2 & 7 & 4 & & \\
III & 6 & 4 & 4 & & \\
IV & 2 & 1 & 1 & & \\
V & 3 & 2 & 0 & & \\
VI & 2 & 0 & 0 & 9.19 & $.50<\mathrm{p}<.70$ \\
VII & 0 & 0 & 0 & & \\
\hline
\end{tabular}

JOB STATUS

Unemployed

$\begin{array}{llll}\text { because of pain } & 12 & 14 & 12\end{array}$

Unemployed

\begin{tabular}{llllll} 
because of other reasons & 3 & 3 & 1 & & \\
Employed & 4 & 1 & 0 & 6.29 & $.10<\mathrm{p}<.20$ \\
\hline
\end{tabular}

BENEFTTS

\begin{tabular}{lrrrrr} 
WAO** $80-100 \%$ & 11 & 11 & 4 & & \\
WAO $<80 \%$ & 2 & 2 & 8 & & \\
ZW*** & 1 & 2 & 1 & & \\
No benefits & 5 & 3 & 0 & 15.60 & $.01<\mathrm{p}<.02$ \\
\hline
\end{tabular}

* ARBI: Arbeid en Beroepenindeling van het Ministerie van Sociale Zaken en Werkgelegenheid. (Classification of Education and Professions of the Dutch Department of Social affairs and Employment). Ranging from elementary (I) to academic (VII).

** WAO: Wet op de Arbeids Ongeschiktheid (Dutch Disablement Insurance Act). Disablement insurance benifits can only be given after being on sick leave for at least 1 year.

*** ZW: Ziektewet (Dutch Health Law). Recipients of ZW receive sick leave benefits. 
Table 9.I. (Cont.) Biographical data I. $\mathrm{CHI}^{2}$ test for independent samples.

OP $\quad$ OC $\quad$ WLC

Unsuccessful use of TENS prior to admission

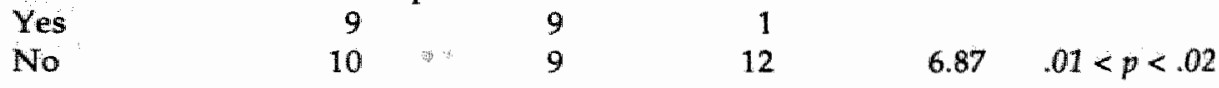

Unsuccessful nerve blocks prior to admission

$\begin{array}{lrrrrr}\text { Yes } & 9 & 8 & 1 & & \\ \text { No } & 10 & 10 & 12 & 6.14 & .01<p<.02\end{array}$

Number of back surgeries prior to admission

$\begin{array}{lrrrrl}0 & 10 & 11 & 5 & & \\ 1-3 & 7 & 7 & 5 & & \\ >3 & 2 & 0 & 3 & 4.78 & .30<\mathrm{p}<.50\end{array}$

Use of supportive equipment

\begin{tabular}{lrrrrr} 
Yes & 5 & 4 & 6 & & \\
No & 14 & 14 & 7 & 2.26 & $.30<\mathrm{p}<.50$ \\
\hline
\end{tabular}

Table 9.II.

Biographical data II. ANOVA.

\begin{tabular}{llrrrr}
\hline & & OP group & OC group & WLC group & F \\
\hline \multirow{2}{*}{ Age } & $\mathrm{X}$ & 43.16 & 39.78 & 46.00 & 2.344 \\
& $\mathrm{SD}$ & 8.02 & 7.55 & 8.53 & \\
& range & $24-55$ & $25-51$ & $33-60$ & \\
Pain duration & $\mathrm{X}$ & 12.58 & 11.06 & 11.15 & 0.161 \\
since onset & $\mathrm{SD}$ & 9.36 & 7.61 & 10.12 & \\
& range & $1-31$ & $2-25$ & $\mathbb{1 - 3 7}$ & \\
Number of back & $\mathrm{X}$ & 1.37 & 0.61 & 1.38 & 1.584 \\
surgeries & $\mathrm{SD}$ & 1.67 & 0.85 & 1.76 & \\
& range & $0-5$ & $0-2$ & $0-5$ & \\
\hline
\end{tabular}

${ }^{*} \mathrm{p}<.05 ; * * \mathrm{p}<.01 ; * * * \mathrm{p}<.001$.

Of the total group, $70 \%$ were married, $53 \%$ received $80-100 \%$ disability income, $25 \%$ received partial disability income, and only $5 \%$ were employed. $38 \%$ of the patients had used TENS-unit, without success, and 36 $\%$ received nerve blocks, also without success. 
Twble 9 m preserts data of sex, age and twil ataws lor the tot low back

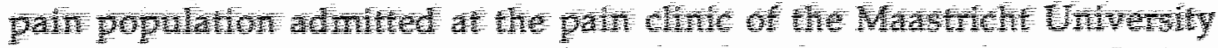

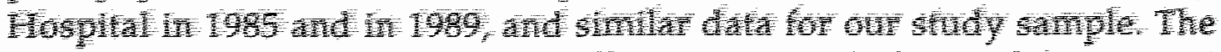
absence of any sigmican group doferences argue in favor of the external williny of our study. Under the condition of acceptable infernal wallity. he study resuts may be generalked wo the pin dinc CLBP population of the Maastiche Unwersity Hospital.

Table 9.II Comparison among the study sample and CLPP populations admi-

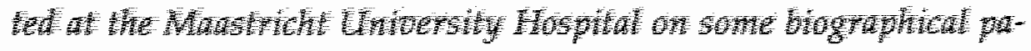
rameters.

\begin{tabular}{|c|c|c|c|c|}
\hline POPUATION & $\begin{array}{l}\text { Univ. Hospilat } \\
\text { Population (1985) }\end{array}$ & $\begin{array}{l}\text { Univ Hospital } \\
\text { Population (1989) }\end{array}$ & $\begin{array}{l}\text { Study Sample } \\
1986-1989\end{array}$ & $\mathrm{CH}$ \\
\hline Wh & 6.5 & 83 & 50 & \\
\hline \multicolumn{5}{|l|}{ SEX } \\
\hline Male & $12 \%$ & $30 \%$ & 24 & \\
\hline Female: & $58 \%$ & 70 㤑 & $76 \%$ & 432 \\
\hline \multicolumn{5}{|l|}{ AVILSTATUS } \\
\hline Married & 35 吆 & $78 \%$ & $70 \%$ & \\
\hline Single & 5 & $22 \%$ & $30 \%$ & 3.60 \\
\hline \multicolumn{5}{|l|}{ ACE } \\
\hline$<31$ & $6 \%$ & $13 \%$ & $10 \%$ & \\
\hline $31=40$ & $20 \%$ & $26 \%$ & $25 \%$ & \\
\hline $4-50$ & 430 & $36 \%$ & $46 \%$ & \\
\hline $51=60$ & $23 \%$ & $13 \%$ & $19 \%$ & \\
\hline$>60$ & $8 \%$ & $11 \%$ & $0 \%$ & 9.98 \\
\hline
\end{tabular}

${ }^{*} \mathrm{p}<.05,{ }^{* *} \mathrm{p}<.01,{ }^{* * *} \mathrm{p}<.001$

\subsubsection{Personality characteristics}

Figures 9.2 and 9.3, showing the mean MMPI-profiles for the male and female patients respectively, reveal that no clinically significant differences exist among the groups. The profiles are similar to profiles of chronic pain patients previously published by several authors (e.g. Hanvik, 1951; Bradley \& Van der Heide, 1984). A MANOVA testing significant differences among the three groups on the 13 clinical MMPI-scales (raw scores) revealed no group effect (Wilk's lambda $=.55, F(26,86)=1.14, p=.320)$. 


\section{MALE}

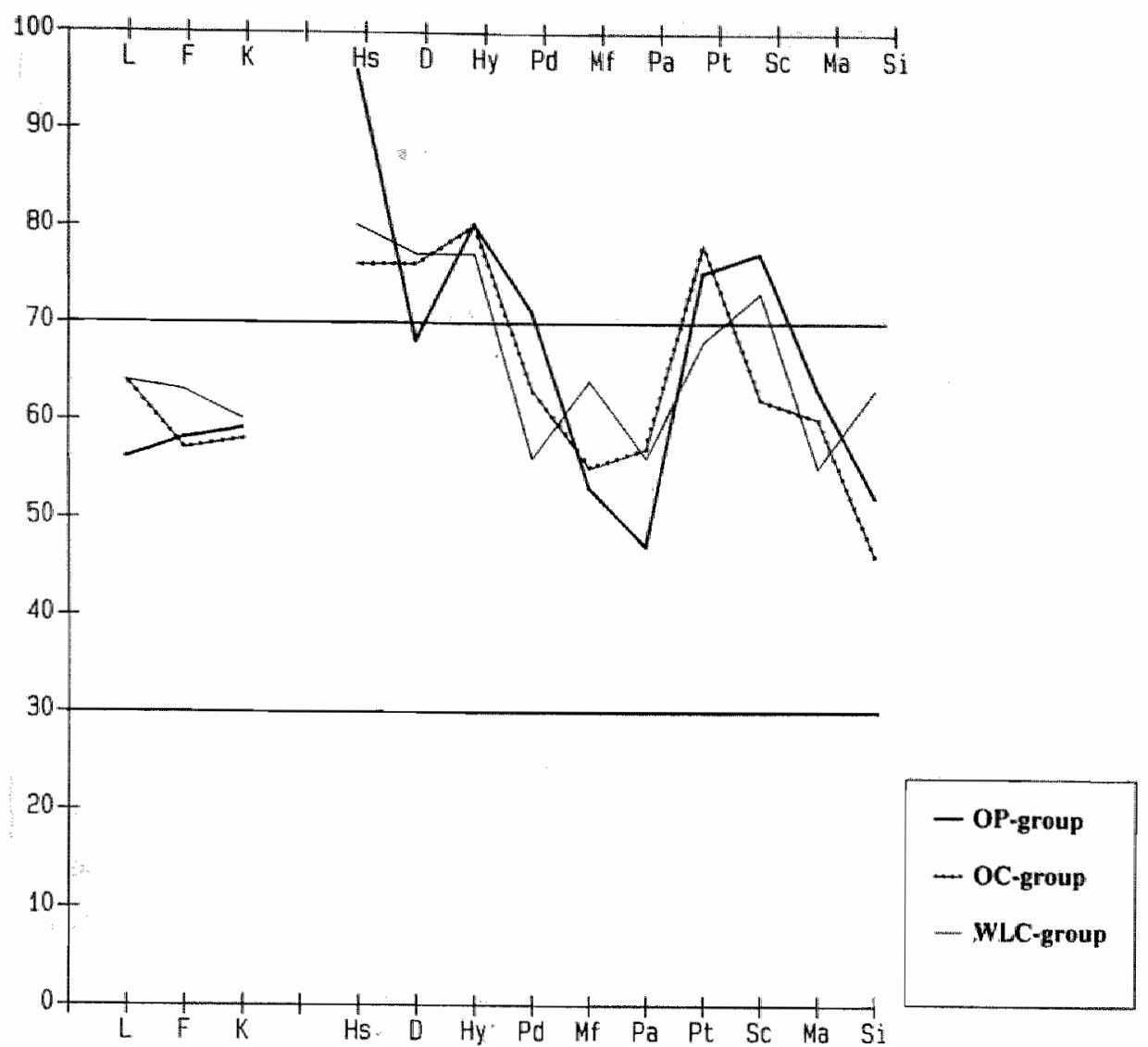

Figure 9.2: Male MMPI-profiles at PRE assessment

\section{.2.5. Outcome measures}

A comprehensive set of pain measures was chosen according to the threesystems model of chronic pain. Because of the lack of adequate instruments, no measures of the physiological response system of pain were included in this study.

For the motoric response system three instruments were used:

1. The Checklist for Interpersonal Pain Behavior (CHIP, see chapter 4) providing five reliable factors: Distorted Mobility (DM), Verbal Complaints (VC), Non-Verbal Complaints (NC), Nervousness (N), and Depression (D). 


\section{FEMALE}

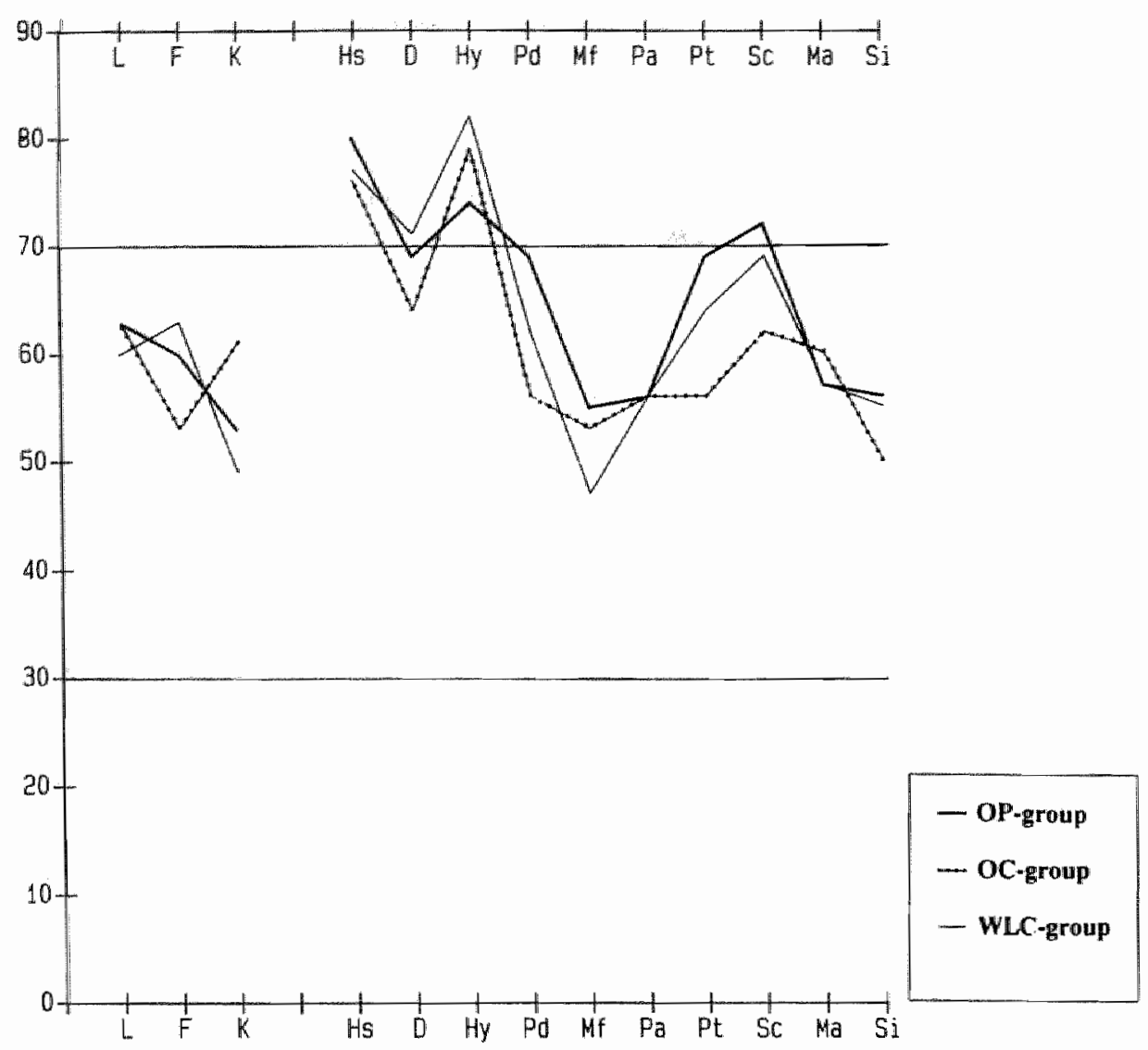

Figure 9.3: Female MMPI-profiles at PRE assessment

2. The Pain Behavior Scale (PBS, see chapter 5) providing one reliable and valid measure of pain behavior.

3.The Motoric Skills Observation Scale (MOSOS, see chapter 6) providing measures for residual health behavior: Walking Distance (WD), number of Arm Rowing movements (AR), number of Leg Rowing movements (LR), sitting tolerance time (SIT), standing tolerance time (STA), number of replaced objects per second (REPL), a swimming performance score (SWIM), and a swimming distance score (SWIMD). A general time score (T) was included that consisted of the sum of the times for walking, bicycling, swimming, arm rowing and leg rowing. The internal consistency for this general time score was high enough (Cronbach's alpha = $.71)$. 
For the verbal-cognitive response system the three following instruments were used:

1. Five factors of the Pain Cognition List ( $P C L$, see chapter 7$)$ are included in this study: Pain impact (PI), Catastrophizing (CA), Outcome efficacy $(\mathrm{OE})$, Acquiescence (AC), and Reliance on Health Care (RH). The PCL was developed as a measure to quantify pain-related attributions and efficacy expectations.

2. The Pain Rating Index (PRI) and the Number of Words chosen (NW) of the McGill Pain Questionnaire - Dutch Version (MPQ-DV, Vanderiet et al., 1986) are widely used measures of the pain experience. For the English version, evidence has supported their reliability and validity (Syrjala \& Chapman, 1984).

3. The Visual Analog Scale (VAS, Huskisson, 1983) is one of the most frequently used measures of the current pain intensity. A 10 centimeter line is provided with anchors at the two extremes: "I feel no pain at all" on the left extreme and "I feel the worst pain ever experienced" on the other.

Another set of measures, which were related to complaints other than pain were taken only once before, once after treatment and once at each of the follow-up assessments. These will be dealt with in chapter 11. Measures of medical consumption (e.g. medication intake and visits to health care providers) are included as well, but results will be reported elsewhere.

\subsubsection{Statistical procedures}

\subsubsection{Variable reduction technique}

Because of the high number of variables compared to the relatively small number of subjects, composite variable scores will be used as dependent variables. The aim is to select for subsequent analyses a set of measures that is least mutually intercorrelated (Shaffer, 1979). In this study, a principal components analysis is applied on the total data set. The data consist of the 23 pain measures obtained at the 10 successive time points (without FU1 and FU2) from 37 subjects, forming a $370 \times 23$ data input matrix of 370 sets of measures (cases) for each of the 23 variables. These data are submitted to a generally available principal components analysis with varimax rotation (SPSS Inc, 1988). Variables with a factor loading less than .50 on all variables are excluded. After transformation of the data into standard $z$ scores, composite scores, as well as factor scores are calculated. The composite scores consist of the sums of standardized individual variable scores for the selected variables. Product-moment correlation coefficients are calcu- 
lated among factor scores and composite scores. If correlations are significantly high, composite scores will be used in subsequent analyses. If not, factor scores will be used.

\subsubsection{Testing the hypotheses}

For our design, Analysis of Variance (ANOVA) for repeated measures is chosen. This ANOVA is a special case of MANOVA, in which the dependent variable is measured at different occasions. The data of the first measurement point are omitted (for the $O P$ and $O C$ group only). For each dependent variable three factors can be defined: a Measurement factor, a Time factor (e.g. PRE, TREATMENT, POST) and a Group factor (e.g. OP, OC, WLC). Each Time factor level consists of the mean of the measurements that belong to that assessment phase. The effects we are interested in are the Time effect (there is a significant change across the assessment phases) and the Group X Time interaction effect (the significant change is different across groups). Two contrasts will be used: the Helmert contrasts and the Reversed Helmert contrasts (Hand \& Taylor, 1987). The Helmert system is one of the most common orthogonal sets of contrasts. Each level is compared with the average of the following levels. The first contrast compares the first level with the second and the third level. The second contrast compares the second with the third level. These contrasts will be used on the Time factor when only the two experimental groups (OP and $O C$ ) are included in the analyses. The Reversed Helmert system is designed to compare levels of a factor with the mean of the previous levels of that factor. The first Reversed Helmert contrast compares the last level with the average of the two previous levels. This contrast is useful to test the hypothesis that the WLC does not differ from the two experimental groups. The second Reversed Helmert contrast compares the second with the first level.

Before statistical procedures are applied on the dependent variables separately, four doubly multivariate repeated-measures ANOVA are performed, during which all dependent variables are considered simultaneously in a factor Variable. For these MANOVA's, the significance level is .05. The Measurement factor is omitted. Each Time factor level consisted of the mean of the three measurements that belong to that Time factor level. The individual successive approximation programs in the operant treatment did not allow the completion of the MOSOS for $30 \%$ of the patients during treatment. Therefore, for composite variables including MOSOS, the Time factor level TREATMENT consists of the 6 th measurement only.

In the first MANOVA, the Group factor consists of the two experimental groeps (OP and OC) and the repeated measures factor was Time (PRE, TREATMENT, and POST). The Null Hypotheses are: 
$\mathrm{Ho}_{1}$ : The dependent variable will not change across assessment phases, $\mathrm{Ho}_{2}$ : This will be the case for both groups.

For the second MANOVA, the three groups, including the WLC, are considered. The Time factor consists of only two levels (PRE, TREATMENT). In this analysis, the Null Hypothesis is:

$\mathrm{Ho}_{3}:$ The change across assessment phases is equal for all three groups.

For the third MANOVA, only the experimental groups are included in the Group factor, and the Time factor now includes the first follow-up measurement (PRE, POST, FU1). Here, the Null Hypotheses are:

$\mathrm{Ho}_{4}$ : There is no difference among PRE, POST and FU1 phases, and $\mathrm{Ho}_{5}$ : This will be the case for both groups.

The fourth MANOVA only deals with the OP group only, and makes a comparison among the post-treatment phase, the 6-month follow-up phase, and the 12-month follow-up phase. At the time when the analyses are made, the 1 year follow-up measurements are not finished for the OCgroup. Therefore, this analysis only includes the OP group. The Null Hypothesis here is stated in the other direction:

$\mathrm{Ho}_{6}$ : There will be a significant difference among POST, FU1 and FU2 phases.

Depending on the results of these overall MANOVA's, ANOVA's for repeated measures will be carried out for each dependent variable separately.

\subsection{Results}

\subsubsection{Variable reduction}

Based on the 'eigenvalue $>1$ ' criterion, the principal component analysis revealed 7 factors. These accounted for $73.9 \%$ of the total variance in the original measures. The term 'factor' will be used interchangeably with the more specific term 'component', in subsequent portions of this chapter. This is consonant with the common usage elsewhere, especially when describing outputs such as factor scores. The communalities of the 23 variables (the proportion of each variable's total variation involved in the components) ranged from 0.59 to 0.90 with an average of 0.70 . 
The 7 components were rotated using the normalized varimax criterion, converging in 13 iterations. Table 9.IV shows the rotated component matrix. Components were labeled based on their variables with highest loadings:

1. Health behavior A:

This component refers to motoric activities, including walking, rowing, standing and replacing objects, and negatively to observed distorted mobility.

2. Pain behavior:

This component reflects pain behaviors, including distorted mobility, verbal and nonverbal complaints.

3. Health behavior $B$ :

Only swimming items of the MOSOS have high loadings on this factor. In addition, acquiescence loads negatively, reflecting an active attitude towards the pain problem.

4. Pain experience:

This factor reflects the intensity of the pain experience as measured by the McGill Pain Questionnaire. Interestingly enough, the VAS related to the current experienced pain intensity has a moderate loading on this factor.

5. Negative affect:

Besides observed nervousness and depression as measured with the CHIP, the VAS appears to covary highly with this factor.

6. Distorted cognitions: Three factors of the PCL belong to this factor. Pain impact, Catastrophizing and Outcome Efficacy. The unexpected covariation of PCLOE with the two other PCL factors makes the validity of this factor doubtful.

7. Reliance on Health care: Only one measure belongs to this factor: PCL Reliance on Health care, reflecting external locus of control in regard to pain management.

In order to assess the stability of the components found, a principal component analysis is also carried out on the data of the first measurement point. The component structure is shown in Table 9.V. This analysis (which 
has to be interpreted with caution because of the unfavorable subjects/ variables ratio) reveals the same number of components, each of which are composed of similar variables as the structure shown in Table 9.IV. Only percentages of explained variance of the respective components are slightly different in both analyses. It can be concluded that the structure found is quite stable, and the variation due to the repeated measures minimal. The component matrix presented in Table 9.V. will be used in further analyses.

After transformation of the total data set into standard $z$-scores, composite scores were calculated. Table 9.VI shows the correlations among the factor scores and the composite scores (based on the selected variables). Correlations range from .82 to .95 , and are all significant beyond the .001 level. Table 9.VI further suggests that the internal consistency (Cronbach's Alpha) of the composite scores is within the acceptable range. Table 9.VII presents the intercorrelations among the composite scores. Of interest is that intercorrelations among variables representing the overt-motoric response system are quite elevated (e.g. pain behaviors - health behavior $\mathrm{A}=$ -.72 ). Intercorrelations among variables representing the verbal-cognitive response system are lower but still significant (e.g. pain experience - pain cognitions $=.28$ ). Unexpectedly, correlations among "reliance on health care' and all other variables are lacking. Intercorrelations among variables representing different response systems are low as well (e.g. pain behaviors - distorted cognitions $=.17$ ). These results underscore the importance of the concept of 'discordance' among the response systems.

Since there is no basis on which to select the one or true measure, the various types of measures may all be true measures that together are useful in painting the picture of the current pain problem.

Figures 9.4 to 9.10 provide the graphical representation of the mean scores on the respective components (=dependent variables) for the groups under study on the 11 measurement points. Visual inspection of these graphical presentations suggest that for all components except 'pain experience', change in the expected direction is seen for both the $O P$ and $O C$ group, while this is not the case for the WLC group. Furthermore, the OC group appears to show slightly more pronounced changes in the expected direction than the OP group. Compared to the POST assessment, the followup data suggest some decline. Also here, the OC group shows better followup results than the OP group, except for the variables 'pain experience' and 'reliance on health care'. Of course, these conclusions are premature, and should be confirmed by statistical analyses.

Visual inspection of figures 9.4 to 9.10 also suggest that for some components, groups have different initial PRE-treatment values, which may 
Table 9.IV. Rotated Component Matrix based on total dataset

COMPONENT

Variable

II

III

IV

$\mathrm{V}$

VI

VII

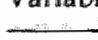
HeBeh A PainBe HeBeh B PainExp NegAff DisCogn RelHC

1. PBS

2. CHIP-DM

3. CHIP-VC

4. CHIP-NC

5. CHIP-N

6. CHIP-D

7. MOSOS-WD

8. MOSOS-AR

9. MOSOS-LR

10. MOSOS-T

11. MOSOS-STA

12. MOSOS-SIT

13. MOSOS-REPL

14. MOSOS-SWIM

15. MOSOS-SWIMD

16. PCL-PI

17. PCL-CA

18. PCL-OE

19. PCL-AC

20. PCL-RH

21. VAS

22. MPQ-PRI

23. $\mathrm{MPQ}-\mathrm{NW}$

$74 \quad-17$

Percentage of

explaüned variance $33.6 \% \quad 9.9 \% \quad 8.1 \% \quad 7.1 \% \quad 5.7 \% \quad 5.9 \% \quad 4.4 \%$

complicate the interpretation of the results. If group differences are found on the PRE assessment phase, and the PRE assessment level is shown to be associated with the change across treatment, analysis of covariance (ANCOVA) might be the analysis of choice. To rule out this possibility, univariate ANOVA's are carried out testing group differences at the PRE asessment phase. Only for component "distorted cognitions", a statistically significant group difference is found when the WLC group is compared with both treatment groups $(F=5.98, p=.018)$. Moreover, for this component significant correlations are found between the PRE assessment phase and both the PRE-TREATMENT change (Pearson $r=0.51, p<.01$ ) and the 
Table 9.V. Rotated Component Matrix based on data of measurement point 1.

COMPONENT

\begin{tabular}{lllllll}
\hline I II & III & IV & V & VI & VII
\end{tabular}

Variable

HeBeh A PainExp HeBeh B PainBe DisCogn NegAff RelHC

1. PBS

2. CHIP-DM

$-.28 \quad .25$

3. CHIP-VC

$\begin{array}{rr}-.28 & .25 \\ -.29 & .42\end{array}$

$-.10 \quad .11$

\begin{tabular}{|l|}
\hline-.30 \\
\hline-.62 \\
\hline-.04 \\
-.28
\end{tabular}

$-.03$

.06

$\begin{array}{ll}-.12 & -.07\end{array}$

5. CHIP-N

$-.00 \quad .05$

6. CHIP-D

7. MOSOS-WD

8. MOSOS-AR

9. MOSOS-LR

10. MOSOS-T

11. MOSOS-STA

12. MOSOS-SIT

13. MOSOS-REPL

\begin{tabular}{rr}
.69 & -.31 \\
\hline 45 & -.20
\end{tabular}

14. MOSOS-SWIM

\begin{tabular}{ll}
.45 & -.20 \\
.78 & -.14 \\
\hline & -.27 \\
\hline
\end{tabular}

$-.11$

\begin{tabular}{ll}
\hline .74 & -.10
\end{tabular}

$\begin{array}{ll}.18 & -.06\end{array}$

15. MOSOS-SWIMD

16. PCL-PI

17. PCL-CA

18. PCL-OE

19. PCL-AC

20. PCL-RH

21. VAS

22. MPQ-PRI

23. MPQ-NW

.79

\begin{tabular}{lr|}
.21 & -.62 \\
.17 & -.59 \\
\hline
\end{tabular}

\begin{tabular}{lr}
.17 & -.59 \\
\hline 26 & -.30
\end{tabular}

$.01 \quad .11$

$.11 \quad .20$

$.06 \quad .18$

$.14 \quad .23$

.58
.63
.62

$-.10$

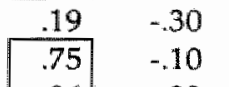

$-15$

$-.16$

$.86 \quad .23$

$\begin{array}{ll}.25 & -.04\end{array}$

$\begin{array}{ll}-.01 & -.06\end{array}$

$\begin{array}{lll}.47 & -.14 & .30\end{array}$

\begin{tabular}{lll}
\hline .63 & .02 & -.19 \\
\hline .19 & -.19 & -.16
\end{tabular}

$\begin{array}{ll}-.19 & -.16\end{array}$

.22

$-.17$

$-.09$

.02

$.82 \quad-.03$

$$
.19
$$

$\begin{array}{lll}.40 & -.04 & .02\end{array}$

$\begin{array}{lll}-.06 & -.12 & -.20\end{array}$

.85

.02

$-.16 \quad-.03$

$.17 \quad .04$

$\begin{array}{rr}.02 & .08\end{array}$

$\begin{array}{lll}.10 & -.22 & -.19\end{array}$

.09

.12

.25

.40

$\begin{array}{lll}.63 & -.22 & -.19 \\ .81 & .20\end{array}$

.12

.16

$.05 \quad-.08$

$-.13 \quad-.27$

$-.27$

$-.02$

$\begin{array}{ll}.76 & -.19 \\ .05 & -.04\end{array}$

$-.27$

$\begin{array}{rr}-.18 & .08 \\ -.12 & -.24\end{array}$

$\begin{array}{ll}.78 & -.18\end{array}$

$-.01$

.08

$-.10$

$\begin{array}{ll}-.12 & -.24\end{array}$

.85

.21

$-.18$

$-.03$

$\begin{array}{rr}.04 & .38\end{array}$

$-.43$

$-.12 \quad-.06$

$-.03$

.29

$.37 \quad .32$

$.11 \quad .13$

.04

.15

\begin{tabular}{lr}
-.38 & -.38 \\
-.12 & .87 \\
\cline { 2 - 2 } & .05
\end{tabular}

.04

.01

.10

$-.12$

$-.11$

.01

Percentage of

\begin{tabular}{lllllll} 
explained variance $28.0 \%$ & $13.1 \%$ & $9.9 \%$ & $8.0 \%$ & $6.9 \%$ & $5.8 \%$ & $4.6 \%$ \\
\hline
\end{tabular}

TREATMENT-POST change (Pearson $r=0.58, p<.01$ ). Thus, an ANCOVA with PRE assessment level as the covariate will be used to test $\mathrm{Ho}_{3}$ for this component. 
Table 9.VI. Product-moment correlation coefficients among factor scores (FACT) and composite scores (COM), and Cronbach's Alpha for the respective components.

COMI COMII COMIII COMIV COMV COMVI COMVII HeBeh A PainBe HeBeh B PainExp NegAff DisCogn RelHC

\begin{tabular}{|c|c|c|c|c|c|c|c|}
\hline FACT I & $87^{* *}$ & $-.44^{* *}$ & $.29^{* *}$ & -.10 & $-.31^{* *}$ & .03 & $.20^{* *}$ \\
\hline FACT II & $-37^{* * 4}$ & $83^{* *}$ & $-.18^{* * *}$ & .03 & $.19 * *$ & .02 & $-.28^{* * *}$ \\
\hline FACT III & $.24^{* * *}$ & -.11 & $89^{* *}$ & -.02 & $-.16^{*}$ & -.04 & -.04 \\
\hline FACT IV & -.10 & .03 & -.03 & $95^{* *}$ & .09 & .20 & -.02 \\
\hline FACT V & $-.17^{* *}$ & $.22^{* *}$ & -.11 & -.01 & $86^{* *}$ & .14 & $-.16^{*}$ \\
\hline FACT VI & -.01 & -.02 & $-.14^{*}$ & $.14^{*}$ & .09 & $92^{* *}$ & -.11 \\
\hline EACT VII & .06 & $-.16^{*}$ & .01 & .03 & .03 & -.04 & $.82^{* *}$ \\
\hline \multicolumn{8}{|l|}{ Cronbach"s } \\
\hline Alpha & .92 & .84 & .77 & .92 & .58 & .62 & - \\
\hline
\end{tabular}

${ }^{*} \mathrm{p}<.01, * * \mathrm{p}<.001$ (one-tailed)

Table 9.VII.Product-moment correlation coefficients among composite scores (COM).

\begin{tabular}{llllllll}
\hline & $\begin{array}{l}\text { COM I } \\
\text { HeBeh A }\end{array}$ & $\begin{array}{l}\text { COM II } \\
\text { PainBe }\end{array}$ & $\begin{array}{c}\text { COM III } \\
\text { HeBeh B }\end{array}$ & $\begin{array}{c}\text { COM IV } \\
\text { PainExp }\end{array}$ & $\begin{array}{c}\text { COM V } \\
\text { NegAff }\end{array}$ & $\begin{array}{c}\text { COM VI } \\
\text { DisCogn }\end{array}$ & $\begin{array}{l}\text { COM VII } \\
\text { RelHC }\end{array}$ \\
\hline COM I & 1.00 & $-.72^{* *}$ & $.50^{* *}$ & $-.19^{* *}$ & $-.52^{* *}$ & -.10 & .03 \\
COM II & & 1.00 & $-.42^{* *}$ & .10 & $.46^{* *}$ & $.17^{* *}$ & -.02 \\
COM III & & & 1.00 & -.08 & $-.38^{* *}$ & $-.25^{* *}$ & .04 \\
COM IV & & & & 1.00 & $.19^{* *}$ & $.28^{* *}$ & .00 \\
COM V & & & & & 1.00 & $.22^{* *}$ & -.02 \\
COM VI & & & & & & 1.00 & -.06 \\
COM VII & & & & & & & 1.00 \\
\hline
\end{tabular}

${ }^{*} \mathrm{p}<.01$, ${ }^{* *} \mathrm{p}<.001$ (one-tailed) 


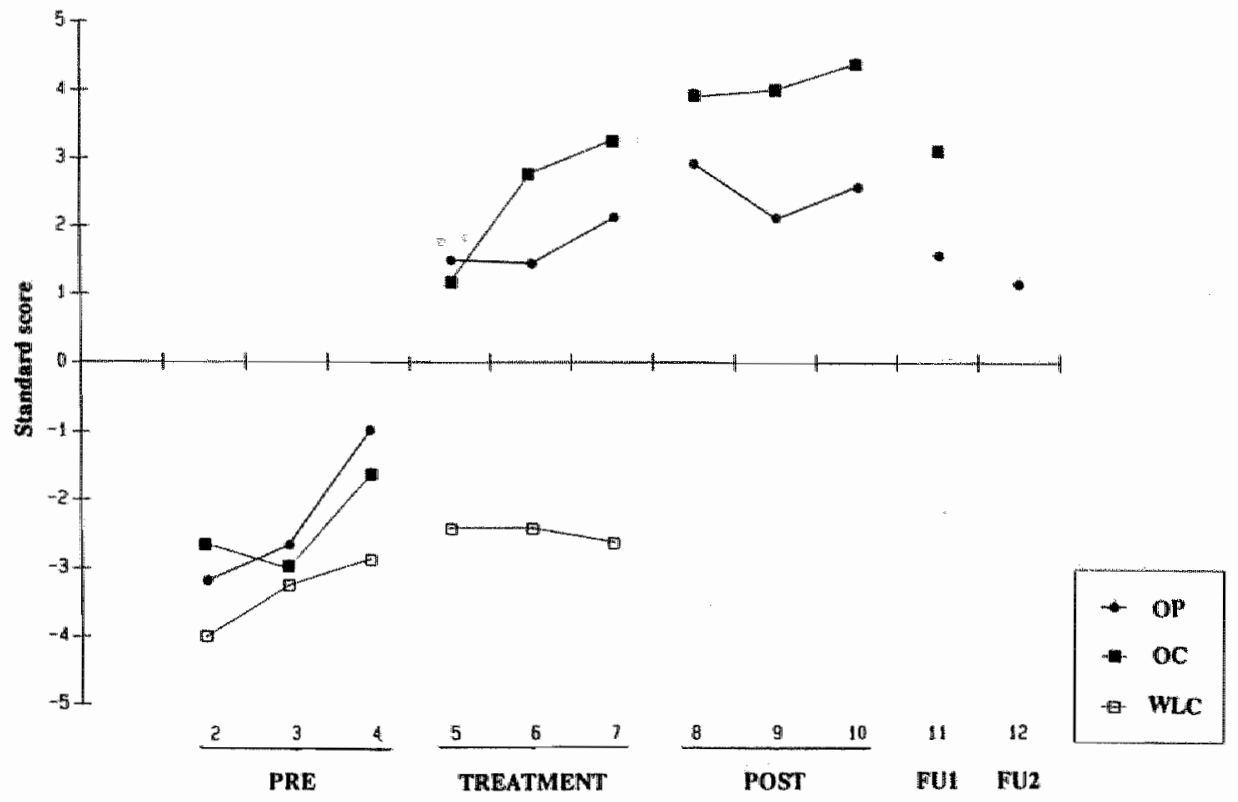

Figure 9.4: Graphical representation of component 'Health Behavior $A$ ' across Time.

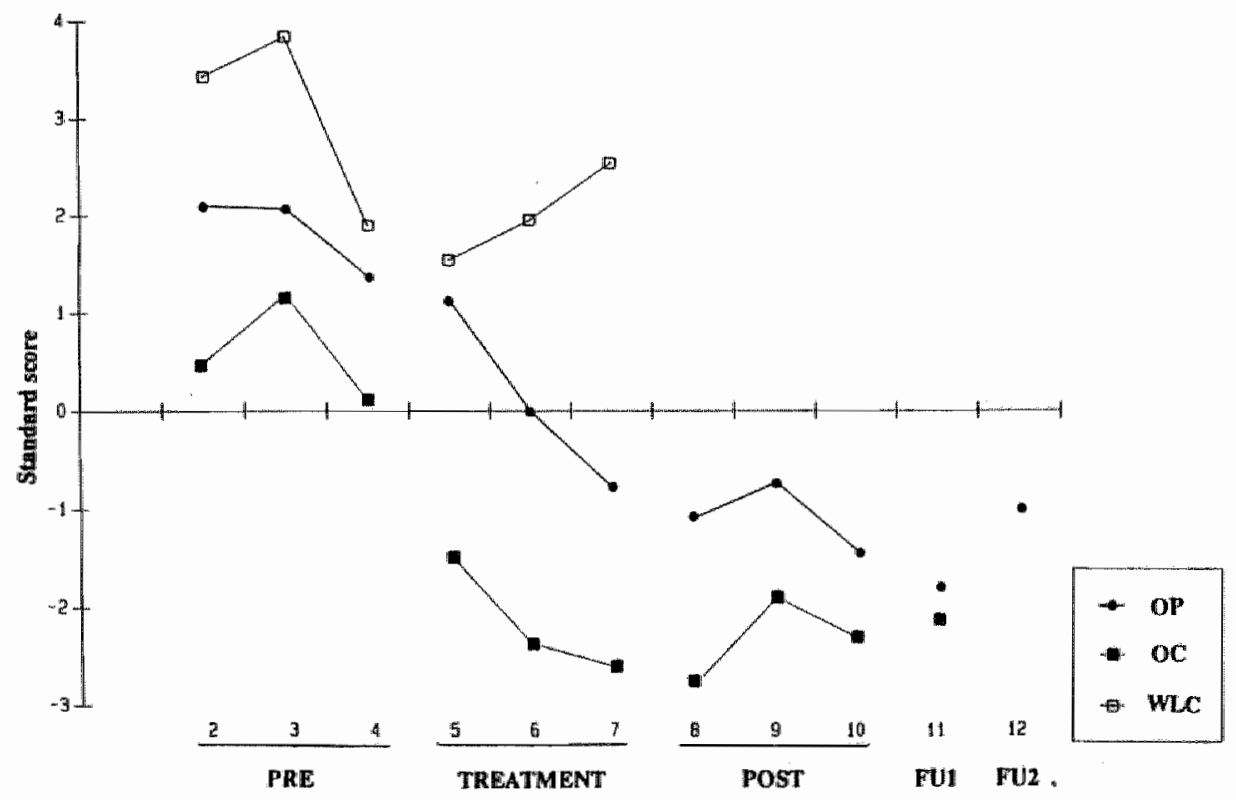

Figure 9.5: Graphical representation of component 'Pain Behaviors' across Time. 


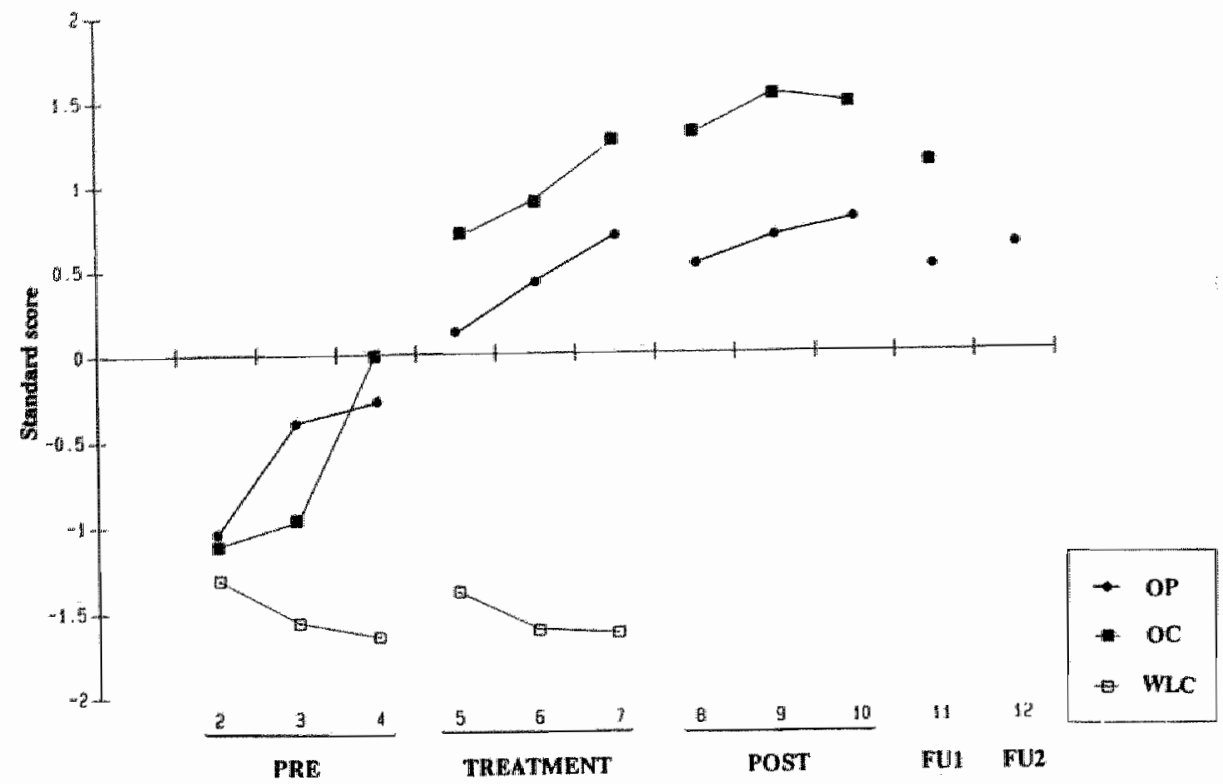

Figure 9.6: Graphical representation of component 'Health Behavior B' across Time.

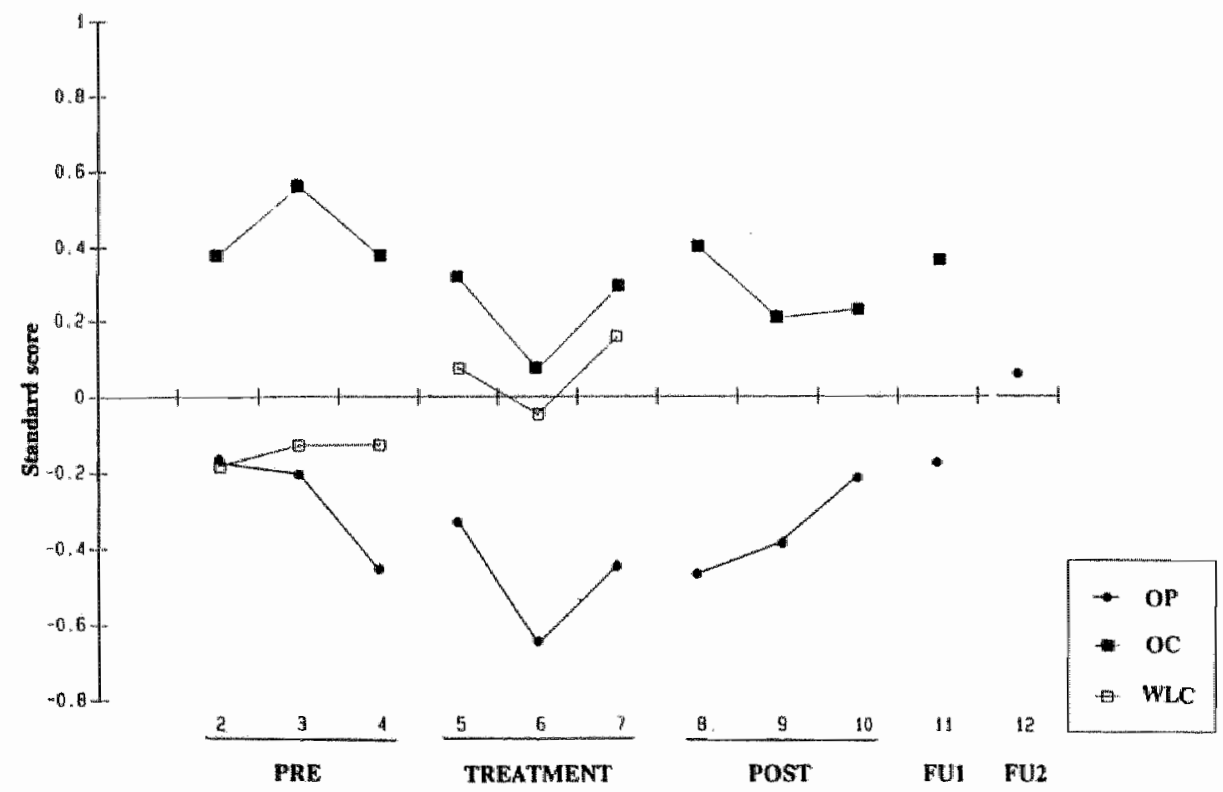

Figure 9.7: Graphical representation of component 'Pain Experience" across Time. 


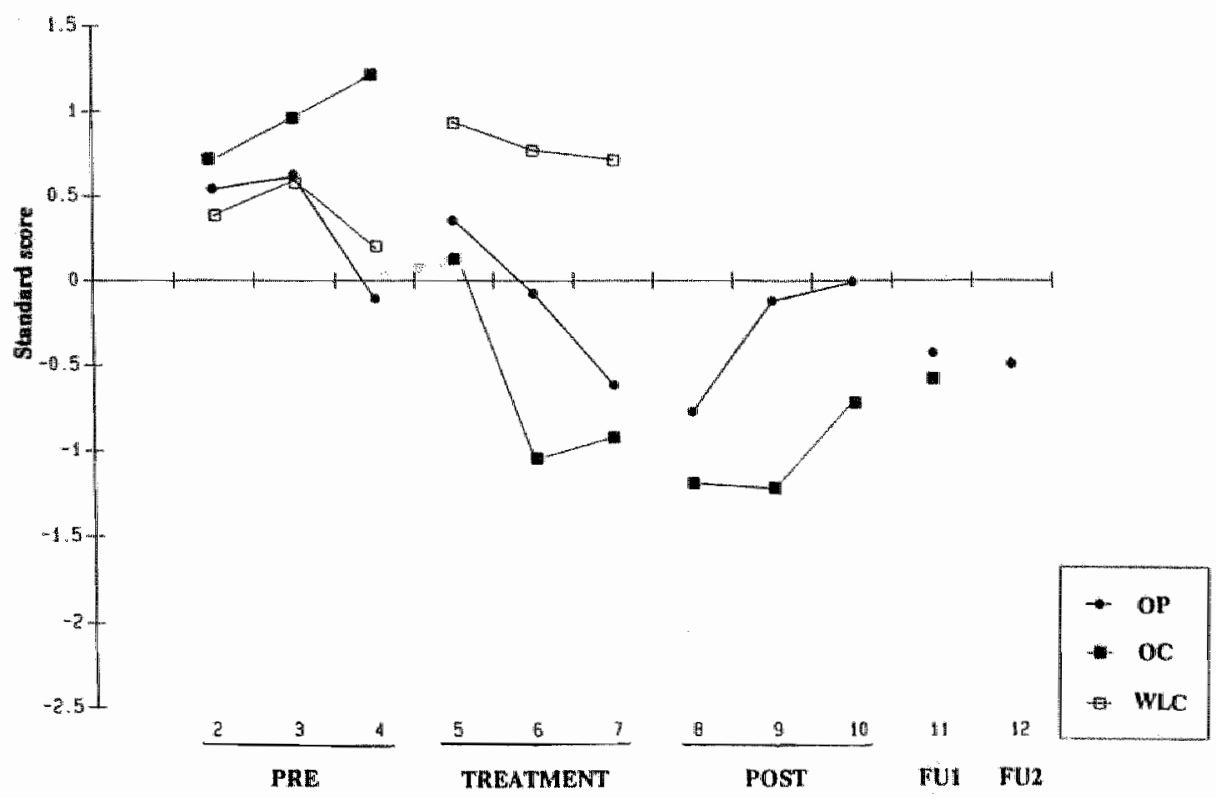

Figure 9.8: Graphical representation of component 'Negative Affect' across Time.

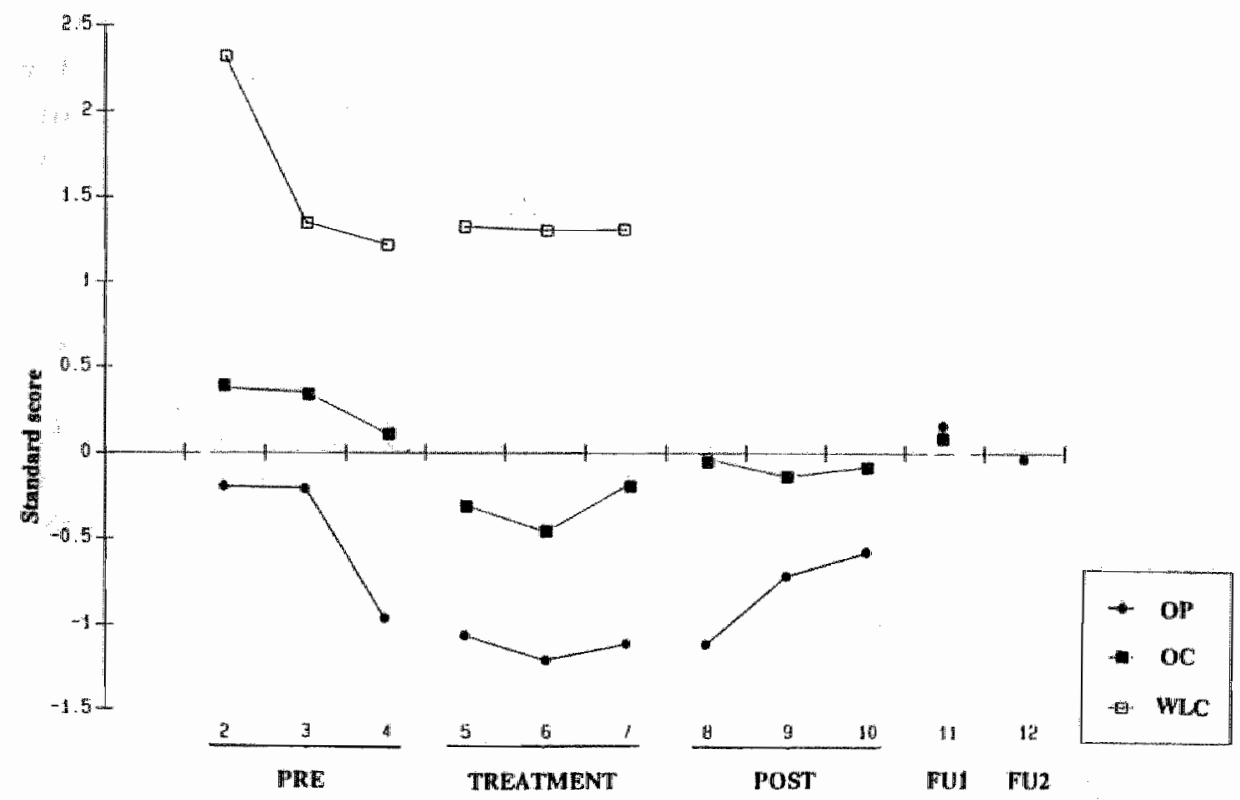

Figure 9.9: Graphical representation of component 'Distorted Cognitions' across Time. 


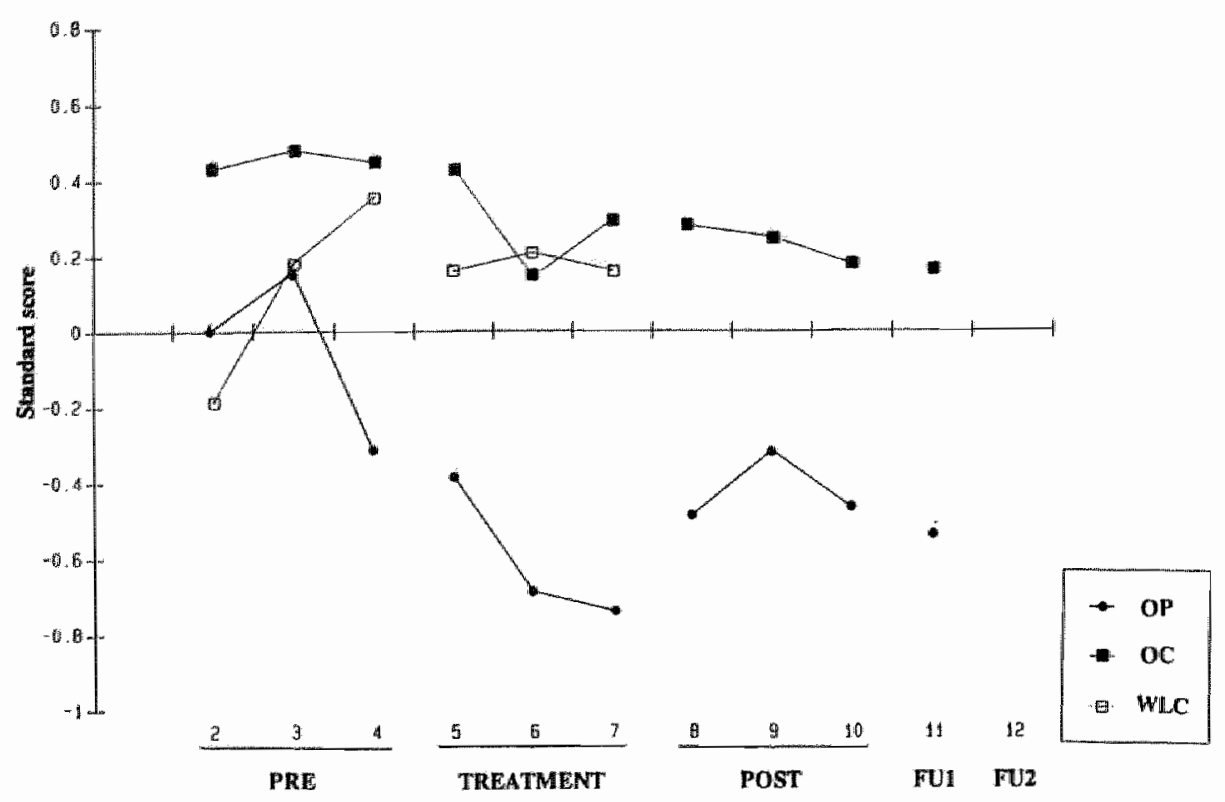

Figure 9.10: Graphical representation of component 'Reliance on Health Care' across Time.

9.3.2. Immediate treatment effects: Comparison between the $O P$ and $O C$ groups. The first doubly multivariate repeated measures ANOVA performed for the two treatment groups revealed a Time effect, and a Variable $X$ Time interaction effect for both contrasts (Table 9.VIII). These results suggest that $\mathrm{Ho}_{1}$ should be rejected: There is a significant change over time, but this change is not the same for all variables. Therefore, examination of the univariate ANOVA's for the dependent variables separately is at issue. $\mathrm{Ho}_{2}$, however, cannot be rejected: In general, the experimental groups do not differ as to the treatment effect across assessment phases.

Separate univariate ANOVA's (Table 9.LX) reveal that for the first Helmert contrast for all but one variable ('Pain Experience'), a significant Time effect is found. For these variables, a significant change in the expected direction is observed after treatment has been started. For the variables 'Health Behavior A' and 'Pain Behavior', a significant Time effect is found on the second Helmert contrast. For these variables the treatment results continue to improve after treatment. For the other variables, the results at the post-treatment assessment are the same as those at treatment assessment. A significant relapse immediately after discharge is not found for any variable. Finally, and for the first Helmert contrast, a Group X Time effect 
Table 9.VIII. Dowbly Multivariate repeated-measures ANOVA for all dependent variables simultaneously on the two experimental groups $O P$ and $O C)$. Comparison among pre, treatment and post assessment phases with Helmert contrasts.

\begin{tabular}{lcc}
\hline Effects & $\begin{array}{l}\text { Wilks } \\
\text { Lambda }\end{array}$ & Approx. F. \\
\hline Constant & & 2.09 \\
Group & .856 & 0.01 \\
Variable & .676 & 0.81 \\
Group X Variable & & 2.32 \\
Time & & \\
1st Helmert (1 vs. 2,3) & & $87.33^{* * *}$ \\
2nd Helmert (2 vs. 3) & & $7.70^{* * *}$ \\
Group X Time & & \\
1st Helmert (1 vs 2,3) & & 2.07 \\
2nd Helmert (2 vs 3) & & 0.29 \\
& & \\
Variable X Time & .222 & $16.92^{* * *}$ \\
1st Helmert (1 vs 2,3) & .523 & $4.40^{* *}$ \\
2nd Helmert (2 vs 3) & & \\
Group X Variable X Time & .776 & 1.44 \\
1st Helmert (1 vs 2,3) & .763 & 1.48 \\
2nd Helmert (2 vs 3) &
\end{tabular}

${ }^{*} \mathrm{p}<.05,{ }^{* *} \mathrm{p}<.01,{ }^{* * *} \mathrm{p}<0.001$

is found for 'Health Behavior B' and 'Negative Affect': Figures 9.6 and 9.8 suggest that the OC-group does better than the OP-group.

\subsubsection{Immediate treatment effects: Comparisons among the three groups under study.}

An analysis similar to that performed for the two treatment groups was also performed for the 2 experimental groups and the WLC group, for which the time factor included only two levels (PRE, TREATMENT). MANOVA revealed several effects for the first Reversed Helmert contrast (Table 9.XI). $\mathrm{Ho}_{3}$ should be rejected: There is a significant change over time, but this change is not the same for all variables. The WLC-group scores change differently over time than the treatment groups scores (Group X Time effect). Moreover, the difference among the groups is not the same 
for all variables (Group X Variable effect), and the change over time is also different for different variables (Group $X$ Variable $X$ Time effect). Also here, these results underscore the importance of univariate ANOVA's for the dependent variables separately.

When looking at the variables separately (Table 9.X), a Group X Time effect for the first Reversed Helmert contrast was lacking for the variables 'Pain Experience', and 'Distorted Cognitions'. For these variables, the change across assessment phases observed in the WLC group is not significantly different from the change observed in the experimental groups. However, as mentioned before, this can be due to the group difference during PRE assessment phase for component 'Distorted Cognitions'. An ANCOVA with the first measurement as covariate reveals a significant Group X Time effect. The regression effect is highly significant, suggesting that a great proportion of the variability is attributable to the covariate. Thus, the rejection of $\mathrm{Ho}_{3}$ only holds for all variables except 'Pain Experience'.

Table 9.IX. Univariate repeated-measures ANOVA for the dependent variables. F-values for comparison among PRE, TREATMENT, and POST assessment phases for the two experimental groups (OP and $O C$ ) with Helmert contrasts.

\begin{tabular}{|c|c|c|c|c|c|c|c|}
\hline Effects & HeBeh A & PainBe & HeBeh B & PainExp & NegAff & DisCogn & RelHC \\
\hline Group & 0.13 & $4.41^{*}$ & 0.34 & 1.20 & 0.12 & 1.84 & $7.09^{*}$ \\
\hline \multicolumn{8}{|l|}{ Time } \\
\hline 1st Helmert & & & & & & & \\
\hline $\begin{array}{l}\text { (1 vs } 2,3 \text { ) } \\
\text { 2nd Helmert }\end{array}$ & $61.42^{4 * *}$ & $62.91 *$ & $110.36^{* * * *}$ & 1.10 & $17.09 * * *$ & $7.66^{* *}$ & $8.75^{* *}$ \\
\hline (2 vs 3 ) & $10.79^{* 4}$ & $8.42^{* *}$ & 0.42 & 0.29 & 1.70 & 3.66 & 1.04 \\
\hline \multicolumn{8}{|l|}{$\begin{array}{l}\text { Group X Time } \\
\text { 1st Helmert }\end{array}$} \\
\hline $\begin{array}{c}\text { (1 vs } 2,3 \text { ) } \\
\text { 2nd Helmert }\end{array}$ & 1.21 & 0.62 & $5.81^{*}$ & 0.02 & $4.69^{\circ}$ & 0.01 & 1.88 \\
\hline (2 vs 3$)$ & 1.97 & 3.67 & 0.84 & 0.60 & 0.28 & 0.46 & 2.66 \\
\hline
\end{tabular}

${ }^{*} \mathrm{p}<.05^{* * *} \mathrm{p}<.01, * * * \mathrm{p}<.001$ 
Table 9.X. Univariate repeated-measures ANOVA for the dependent variables. F-values for comparison among the three groups (OP, OC and WLC) with Reversed Helmert contrasts.

Effects

HeBeh A PainBe HeBeh B PainExp NegAff DisCogn DisCogn\# RelHC

Regression

$14.64^{* * *}$

Group

1st Rev. Helmert

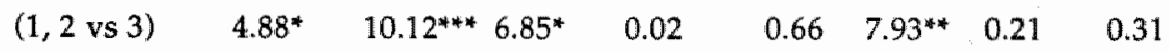

2nd Rev. Helmert

$\begin{array}{lllllllll}\text { (1 vs 2) } & 0.09 & 5.44^{*} & 0.11 & 1.82 & 0.00 & 1.50 & 1.42 & 6.61^{*}\end{array}$

Time

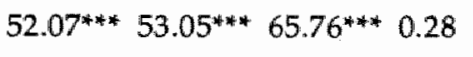

$5.26^{*} \quad 7.62^{* *} \quad 6.15^{*} \quad 6.15^{*}$

Group X Time

1st Rev. Helmert

$\begin{array}{llllll}(1,2 \text { vs } 3) & 14.51^{* * *} 4.39^{*} & 36.63^{* *} 2.78 & 7.27^{* *} 0.92 & 6.08^{*} & 4.35^{*}\end{array}$

2nd Rev. Helmert

$\begin{array}{lllllllll}\text { (1 vs 2) } & 1.46 & 3.07 & 5.23^{*} & 0.01 & 4.36^{*} & 0.05 & 0.46 & 4.07^{*}\end{array}$

\# F-values for ANCOVA with first measurement as constant covariate.

${ }^{*} \mathrm{p}<.05,{ }^{* *} \mathrm{p}<.01,{ }^{* * *} \mathrm{p}<.001$

9.3.4. Long-term treatment effects: Six months follow-up.

Although the analyses provided evidence that the two treatment groups experienced significant change from pretreatment to posttreatment, a doubly multivariate repeated-measures ANOVA, including the 6-month follow-up assessment (PRE, POST, FU1) was performed (Table 9.XII). For the first Helmert contrast, a Time effect and a Time $X$ Variable effect is found. This suggests that the results obtained at post-treatment and 6-mont followup significantly differ from those obtained at pre-treatment, but that this is not the case for all variables. Indeed, it is likely that for the variables, for which no immediate Time effect is found, the 6-month follow-up scores will not differ from the pre-treatment scores. This assumption is underscored by the absence of any effect for the second Helmert contrast, suggesting that for all variables, the results obtained at 6-month follow-up do not differ from those obtained at post-treatment. The results lead to the rejection of $\mathrm{Ho}_{4}$. In other words, regardless of the immediate time effect, the effect remains at 6-month follow-up. 
Table 9.XI. Doubly Multivariate repeated-measures ANOVA for all dependent variables simultaneously on the three groups (OP, OC and WLC). Comparison between the three groups with Reversed Helmert contrasts.

\begin{tabular}{lll}
\hline Effects & $\begin{array}{l}\text { Wilks } \\
\text { Lambda }\end{array}$ & Approx. F. \\
\hline
\end{tabular}

Group

1 st Rev. Helmert (1,2 vs 3$)$

2nd Rev. Helmert (1 vs 2)

Variable

Time

Group X Variable

1st Rev. Helmert (1,2 vs 3$)$

2nd. Rev. Helmert (1 vs 2)

Group X Time

1st Rev. Helmert (1,2 vs 3$)$

2nd Rev. Helmert ( 1 vs 2)

Variable X Time

.348

.598

.807

.870

.691

Group $X$ Variable $\times$ Time

1st Rev. Helmert (1,2 vs 3)

2nd Rev. Helmert (1 vs 2)

.739
$9.64^{* *}$

0.00

1.04

$77.50^{* * *}$

3.13*

$2.48^{*}$

$23.86^{\text {*4* }}$

3.09

$13.09^{* * *}$

${ }^{*} \mathrm{p}<.05,{ }^{* 4} \mathrm{p}<.01,{ }^{* * *} \mathrm{p}<.001$

For both contrasts, no significant Group X Time effect has been found, leading to the rejection of $\mathrm{Ho}_{5}$ : the persistence of the gains holds for both treatment groups.

9.3.5. Long-term treatment effects: One year follow-up.

A final doubly multivariate repeated-measures ANOVA was performed including both follow-up assessments (POST, FU1, FU2), but only for the OP-group (Table 9.XIII). For both Helmert contrasts, no Time effect is found, allowing a rejection of $\mathrm{Ho}_{6}$. These results suggest that the data at the 1 year follow-up measurement do not differ from the data of the post-treatment and of the 6-month follow-up measurement: If there are treatment gains, 
Table 9.XII. Doubly Multioariate repeated-measures ANOVA for all dependent variables (except pain experience) simultaneously on the two experimental groups (OP and OC). Comparison among PRE, POST and FU1 assessment phases with Helmert contrasts.

\begin{tabular}{lcc}
\hline Effects & $\begin{array}{l}\text { Wilks } \\
\text { Lambda }\end{array}$ & Approx. F. \\
\hline Group & & 0.00 \\
Variable & .924 & 0.41 \\
Group X Variable & .893 & 0.60 \\
Time & & \\
1st Helmert (1 vs 2,3) & & $61.75^{* * * *}$ \\
2nd Helmert (2 vs 3) & & 0.49 \\
Group X Time & & \\
1st Helmert (1 vs 2,3) & & 1.57 \\
2nd Helmert (2 vs 3) & & 0.09 \\
Variable X Time & & \\
1st Helmert (1 vs 2,3) & .286 & $12.44^{* * * *}$ \\
2nd Helmert (2 vs 3) & .733 & 1.82 \\
Group X Variable X Time & & \\
1st Helmert (1 vs 2,3) & .821 & 1.09 \\
2nd Helmert (2 vs 3) & .781 & 1.40 \\
\hline
\end{tabular}

${ }^{*} \mathrm{p}<.05, * * \mathrm{p}<.01, * * * \mathrm{p}<.001$

they seem to persist to 1 year after discharge. There are no reasons to believe that this would not be true for the OC-group.

\subsection{Discussion}

In this study, the effectiveness of an operant (OP) and an operant-cognitive (OC) treatment for CLBP was examined on the basis of statistical significance. The OP treatment aimed at reducing pain behaviors and increasing health behaviors and activity levels. In addition, the $O C$ treatment also aimed at reducing distorted pain cognitions. None of the treatments was aimed at reducing reported pain levels. The $O P$ and $O C$ treatments were provided in succession, and patients were admitted to the rehabilitation center in 
Table 9.XIII. Doubly Multivariate repeated-measures ANOVA for all dependent variables (except pain experience) simultaneously on the OP-group. Comparison among POST, FU1, and FU2 assessment phases with Helmert contrasts.

\begin{tabular}{lll}
\hline Effects & $\begin{array}{l}\text { Wilks } \\
\text { Lambda }\end{array}$ & Approx. F. \\
\hline Variable & .915 & 0.30 \\
Time & & \\
1st Helmert (1 vs 2,3) & & 0.65 \\
2nd Helmert (2 vs 3) & & 1.46 \\
Variable X Time & & \\
1st Helmert (1 vs 2,3) & .636 & 1.60 \\
2nd Helmert (2 vs 3) & .840 & 0.53 \\
\hline
\end{tabular}

$* \mathrm{p}<.05, * * \mathrm{p}<.01, * * * \mathrm{p}<.001$

groups of 3 to 4 patients. A waiting list control group was included. The three groups were comparable as to several biographic and personality variables. Most patients met criteria of the Chronic Pain Syndrome, and suffered from persistent low back pain complaints. The attrition rate was low (11\%).

A repeated measures design was chosen. The outcome measures of this study were chosen according to the three-systems model of chronic pain. A variable reduction technique consisted of a principal component analysis applied to the total data set. The analysis resulted in 7 components serving as reliable and valid dependent variables. Four components (health behavior $A$ and $B$, pain behavior, negative affect) represent aspects of the overtmotoric response system and 3 components (pain experience, distorted cognition, reliance on health care) reflect aspects of the verbal-cognitive response system of the CPS. Measures of the physiological response system were absent because adequate assessment instruments were not available.

Both the OP and the OC treatments generally resulted in short-term and long-term improvement on both the motoric as some of the cognitive dimensions of the chronic low back pain problem. As compared with the WLC group, pain behaviors, negative affect, distorted cognition and reliance on health care decreased, and health behaviors increased significantly in both treatment groups. "The reported pain experience did not change, but this was not the aim of treatment. 
Very subtle differences are observed between both treatment groups. Instead of a decrease in distorted cognitions, in decrease in negative affect was more pronounced in the OC group, as well as an increase in some health behaviors (swimming). In the cognitive treatment, patients learned through deep concentration (self-hypnosis) how to modify the modality and locus of the pain, and to focus on other, less painful parts of their body. As found by others (Udolf, 1981), these techniques not necessarily modify the pain experience as such, but result in the patients being less bothered by it. This may explain why OC patients appear less distressed after the treatment than the OP patients.

Drawbacks of the study include the lack of a measure for hypnotic susceptibillity or imagery skills, and the lack of a placebo control group. Hypnotic susceptibility refers to the characteristic of a subject determining the ability to be hypnotized and to attain a given depth of trance (Udolf, 1981). It might be possible that a difference in susceptibility occurred in both treatment groups. If by chance the OC group consisted of more lowsusceptibles, its subjects might have been less responsive to the cognitive treatment. On the other hand, the value of hypnotic susceptibility still is subject of controversy, and standardized hypnotizability measures should not be overestimated (Spinhoven, 1989; James et al. 1989).

This study was not placebo-controlled. As a result, the study results described in this chapter can only be attributed to the whole treatment package (including a-specific factors) and not exclusively to the behavioral principles characterizing both treatments. However, in clinical research, the inclusion of a placebo treatment control condition is hard to accomplish. The researcher is not only faced with ethical considerations of withholding treatment from patients, but also with payment agreements with insurance carriers.

As no changes in pain intensity (as measured with the McGill Pain Questionnaire) occur in any group, it might be argued that the observed changes were not due to decreased pain experience. This implies that patient's disability can be modified in a favorable way, regardless of the pain experience. Both behavioral rehabilitation treatments, as described in appendices $A$ and $B$, result in patients being more active, showing less pain behavior, appearing less distressed, reporting less pain cognitions, and relying less on the health care system. Moreover, these effects are maintained during follow-up. 



\section{CHAPTER 10}

\section{Effectiveness of an operant and an operant-cognitive treatment for chronic low back pain II: clinical significance}

\subsection{Introduction}

A characteristic of most of the existing clinical treatment outcome studies is their reliance on statistical significance to determine the existence of the effects of various interventions. Some authors have openly resented this approach and argued in favor of what is called 'clinical significance' (Barlow et al., 1984; Turner \& Romano, 1984). The important issue in this controversy is the size of the experimental effect with which one is dealing. Statistical significance does not necessarily correspond with the importance of, or the size of the effect. Moreover, and as Barlow et al (1984) pointed out, intersubject variability can also account for a tendency to underestimate clinical effectiveness of a given treatment when relying on statistical significance to determine the presence of an effect. For example, a given treatment might be effective for a few clients, while the remaining clients do not show substantial improvement. In this case, statistics will average out the clinical effect along with changes due to unwanted sources of variability.

Generally, two questions can be raised in the context of clinical significance. The first question is whether the magnitude of change, regardless of statistical significance, has clinical relevance. Secondly, has the treatment really been successful in achieving treatment goals, regardless of the magnitude of change. In this chapter, an attempt is made to examine the clinical significance of the treatment effects by focusing on both questions. Clinical significance will be examined in three ways:

1. For each outcome measure, a minimal level that is considered clinically relevant can be established on the basis of clinical judgement. Such crite- 
ria always tend to be somewhat arbitrary. Therefore 2 more ways of determining clinical significance are chosen.

2. The study results can be compared with data of a relevant reference group, if they are available. Post treatment levels might be considered clinically relevant when they correspond with the level achieved by the lower $30 \%$ of the reference group.

3. Finally, the results obtained can be compaired with data of pain-free controls. Results can be considered clinically relevant when they correspond with 'normal' levels.

Clinical significance of the follow-up levels will be analyzed, and difference in clinical significance between both experimental groups examined as well.

\subsection{Examination of clinically significant pre-treatment post-treatment change}

\subsubsection{Method}

As the 7 dependent variables, described in the previous chapter, are composite scores, the clinical significance will be established on the level of the individual variables which have been entered in the principal component analysis (see Table 9.III.). For each component, the two highest loading variables are selected for examination of clinical significance. Criteria for significant change are established for these 13 variables (MOSOS-WD, MOSOS-LR, CHIP-VC, CHIP-NC, MOSOS-SWIM, PCL-AC, MPQ-PRI, MPQNW, CHIP-N, CHIP-D, PCL-CA, PCL-PI, and PCL-RH. For the PCL and CHIP subscales, for which norms (based on a chronic back pain population) are available, a difference of $15 \mathrm{~T}$-scores in the expected direction will be considered clinically significant. For the MOSOS subscales, the clinically significant increments are more or less arbitrarily chosen, based on clinical experience and practical implications. These criteria are shown in Table 10.I. For each patient the PRE-POST difference score is compared with the criteria for each measure. Patients who meet the criteria for 7 or more variables will be considered 'good responders'. Patients who meet the criteria for more than 4 but less than 7 variables will be considered 'moderate responders", and patients who meet the criteria for less than 4 variables will be considered "poor responders".

The difference between the OP and OC group, in terms of number of patients that are assigned to the three groups of responders, is examined by means of $\mathrm{CHI}^{2}$ tests. The Null Hypothesis is: 
$\mathrm{Ho}_{7}$ : There is no difference between the OP and the OC groups in terms of the number of responders.

\subsubsection{Results}

Results of $\mathrm{CHI}^{2}$ test, examining the difference between the OP and $\mathrm{OC}$ group are summarized in Table 10.I., the mean percentage of responders is $34,5 \%$, for the OP group and $44,4 \%$ for the OC group. For PCL-OE, significantly more OC subjects meet the criteria than OP-subjects. For CHIP-VC, the opposite is true. Considering the classification into good, moderate and poor responders, more good responders are found in the $O C$ group than in the OP-group ( $44.5 \%$ vs. $15.8 \%$ ). Overall, however, $\mathrm{Ho}_{7}$ cannot be rejected: there is no difference between the groups for the three classes $\left(\mathrm{CHI}^{2}=4.588, \mathrm{p}=.100\right)$.

\subsection{Examination of clinically significant post-treatment level}

\subsubsection{Method}

A similar method as described in the previous section is used to examine the clinical significance of the post-treatment level. For the MPQ, however, no acceptable Dutch norms exist. For the 11 remaining variables, criteria for clinically significant levels are developed. For the CHIP and PCL. subscales, norms (based on a chronic pain population, described in paragraph 4.1.2. and 7.2.2. respectively) are available. The criterion for significant post-treatment level is a corresponding T-score of 30 or less (i.e. $70 \%$ of the reference group has a score higher than the criterion score).

For the MOSOS subscales, data from a pain-free sample (PF group) are available. This sample has been used to establish the discriminative value of the MOSOS parts Armrowing, Legrowing, Swimming score, Swimming distance, and a composite Time score including time scores of bicycling, armrowing, legrowing, and swimming distance respectively (see paragraph 6.7.1.). An ANOVA testing mean ages by group (OP, $O C$, and $P F$ ) and sex (male vs. female) revealed no significant differences (groups: $F=1.525, p=.226$; sex: $F=0.004, p=.949$; group $X$ sex: $F=0.803, p=.453$ ). The only difference is that the PF norm group has more male subjects than the experimental groups $\left(\mathrm{CHI}^{2}=6.399, \mathrm{p}=.041\right)$. As males tend to produce higher scores on MOSOS than females (cf. Table 6.VI.), 'normal' criteria are set higher than would be necessary. The criteria for clinically relevant post-treatment levels for MOSOS will be one standard deviation below the level of the PFsample. For MOSOS-WD, a post treatment score of 400 (meter) will be considered significant. The MPQ, for which no norms are available, will be 
omitted from this analysis. Patients who meet the criteria for 8 or more variables will be considered "good achievers". Patients who meet the criteria for more than 4 but less than 8 variables will be considered "moderate achievers", and patients who meet the criteria for less than 4 variables will be considered "poor achievers". The difference in clinically relevant posttreatment level between the OP and $O C$ group is examined by means of $\mathrm{CHI}^{2}$ tests. The Null Hypothesis is stated as follows:

$\mathrm{Ho}_{\mathrm{g}}$ : There is no difference between the OP and the $\mathrm{OC}$ groups as to the number of achievers.

For MOSOS subscales, ANOVA's, with the reversed Helmert contrast for the group factor (OP, OC and PF groups) will be used to test the following Null Hypothesis:

$\mathrm{Ho}_{9}$ : There is a significant difference among the three groups at POST assessment.

In this case the univariate ANOVA's for the dependent variables separately are of special interest, to examine on which variables the experimental groups have reached normal (pain-free) levels. These ANOVA's will be carried out for the data on PRE, POST, FU1 and FU2 assessment phases.

\subsubsection{Results}

Figures 10.1 and 10.2 show the T-score profiles for the PCL and CHIP respectively. It is surprising that, as compared to their respective pain patient-norm groups, our sample groups score relatively low on the PCL and $\mathrm{CHIP}$, indicating a clinically relevant level. Rather unexpectedly, the pre-treatment levels were relatively low as well (all below $\mathrm{T}=70$ level, and for some scales even below $T=50$ level). For PCL-OE, for which an increase is expected due to the treatment, the OC-group achieves a normal level $(\mathrm{T}=46)$.

The results of $\mathrm{CHI}^{2}$ test, examining the difference between the OP and OC group are summarized in Table 10.II. The mean percentage of achievers is $49 \%$ for the OP and $58 \%$ for the OC group. Again, more "good" achievers are found in the OC group than in the OP-group ( $50 \%$ vs. $42 \%$ ). Overall, however, $\mathrm{Ho}_{9}$ cannot be rejected: there is no difference between the groups for the three classes of achievers $\left(\mathrm{CHI}_{2}=2.033, \mathrm{p}=.362\right)$. 
Table 10.I. CHI ${ }^{2}$-tests: difference in pre-post change among the $O P$ and $O C$ group for $P C L, C H I P, M O S O S$ and $M P Q$.

\begin{tabular}{|c|c|c|c|c|}
\hline Variable & $\begin{array}{l}\text { Criterion } \\
\text { PRE-POST }\end{array}$ & $\begin{array}{l}\text { Perc } \\
\text { resp }\end{array}$ & $\begin{array}{l}\text { ntage } \\
\text { nders }\end{array}$ & $\mathrm{CHI}^{2}$ \\
\hline PCL-PI & $>4$ & $\begin{array}{l}\text { OP: } \\
\text { OC: }\end{array}$ & $\begin{array}{l}21 \% \\
55 \%\end{array}$ & 2.922 \\
\hline PCL-CA & $>4$ & $\begin{array}{l}\text { OP: } \\
\text { OC: }\end{array}$ & $\begin{array}{l}37 \% \\
39 \%\end{array}$ & 0.016 \\
\hline PCL-OE & $>3$ & $\begin{array}{l}\text { OP: } \\
\text { OC: }\end{array}$ & $\begin{array}{l}10.5 \% \\
44 \%\end{array}$ & $4.287^{*}$ \\
\hline PCL-AC & $>2$ & $\begin{array}{l}\text { OP: } \\
\text { OC: }\end{array}$ & $\begin{array}{l}10.5 \% \\
5.5 \%\end{array}$ & 0.269 \\
\hline PCL-RH & $>2$ & $\begin{array}{l}\text { OP: } \\
\text { OC: }\end{array}$ & $\begin{array}{l}21 \% \\
5.5 \%\end{array}$ & 2.521 \\
\hline CHIP-DM & $>3$ & $\begin{array}{l}\text { OP: } \\
\text { OC: }\end{array}$ & $\begin{array}{l}63 \% \\
72 \%\end{array}$ & 0.056 \\
\hline CHIP-VC & $>2.5$ & $\begin{array}{l}\text { OP: } \\
\text { OC: }\end{array}$ & $\begin{array}{l}32 \% \\
0 \%\end{array}$ & $4.660^{*}$ \\
\hline CHIP-NC & $>2$ & $\begin{array}{l}\text { OP: } \\
\text { OC: }\end{array}$ & $\begin{array}{l}53 \% \\
67 \%\end{array}$ & 0.117 \\
\hline CHIP-N & $>1$ & $\begin{array}{l}\text { OP: } \\
\text { OC: }\end{array}$ & $\begin{array}{l}37 \% \\
67 \%\end{array}$ & 1.783 \\
\hline CHIP-D & $>1$ & $\begin{array}{l}\text { OP: } \\
\text { OC: }\end{array}$ & $\begin{array}{l}42 \% \\
72 \%\end{array}$ & 2.521 \\
\hline MOSOS-WD & $>100$ & $\begin{array}{l}\text { OP: } \\
\text { OC: }\end{array}$ & $\begin{array}{l}53 \% \\
67 \%\end{array}$ & 0.285 \\
\hline MOSOS-LR & $>50$ & OP: & $\begin{array}{l}68 \% \\
78 \%\end{array}$ & 0.073 \\
\hline MOSOS-SWIM & $>8.5$ & $\begin{array}{l}\text { OP: } \\
\text { OP: }\end{array}$ & $\begin{array}{l}53 \% \\
72 \%\end{array}$ & 1.907 \\
\hline MPQ-PRI & $>8$ & $\begin{array}{l}\text { OP: } \\
\text { OC: }\end{array}$ & $\begin{array}{l}10.6 \% \\
16.7 \%\end{array}$ & 0.004 \\
\hline MPQ-WRD & $>3$ & $\begin{array}{l}\text { OP: } \\
\text { OC: }\end{array}$ & $\begin{array}{l}5.3 \% \\
5.5 \%\end{array}$ & 0.001 \\
\hline
\end{tabular}

${ }^{*} \mathrm{p}<.05 ; * * \mathrm{p}<.01 ; * * * \mathrm{p}<.001$ 
Figures 10.3. to 10.7. show the mean scores of MOSOS subscales across time for both treatments groups. The mean score of the PF group is indicated by a straight line. These figures show that at POST assessment, the treatment groups reach the PF group level, and that the $O C$ group does somewhat better than the OP group. For the measure MOSOS swimming distance, the mean POST assessment level of the treatment groups exceeds the "normal" level.

Table 10.III. reveals that at PRE assessment, the OP and OC groups significantly differ from the PF group. At POST assessment, however, these differences disappeared, suggesting that the patients did as well as the pain-free normals.

Table 10.III. F-values of univariate ANOVA's for MOSOS subscales comparing means of $O P, O C$ and $P F$ groups with the first Reversed Helmert contrast $(1,2$ vs 3$)$.

\begin{tabular}{lllll}
\hline & \multicolumn{4}{c}{ Assessment phase } \\
\cline { 2 - 5 } Variable & PRE & POST & FU1 & FU2 \\
\hline MOSOS-T & $91.61^{* * *}$ & 2.61 & $8.50^{* *}$ & $6.04^{*}$ \\
MOSOS-AR & $65.47^{* * *}$ & 0.01 & 0.61 & 3.40 \\
MOSOS-L & $127.54^{* * *}$ & 1.92 & $8.94^{* *}$ & $11.41^{* *}$ \\
MOSOS-SWIM & $12.93^{* * *}$ & 0.07 & 0.61 & 0.04 \\
MOSOS-SWIMD & $30.02^{* * *}$ & 1.08 & 0.17 & 0.03 \\
\hline
\end{tabular}

${ }^{*} \mathrm{p}<.05,{ }^{* *} \mathrm{p}<.01{ }^{* * *} \mathrm{p}<.001$. 


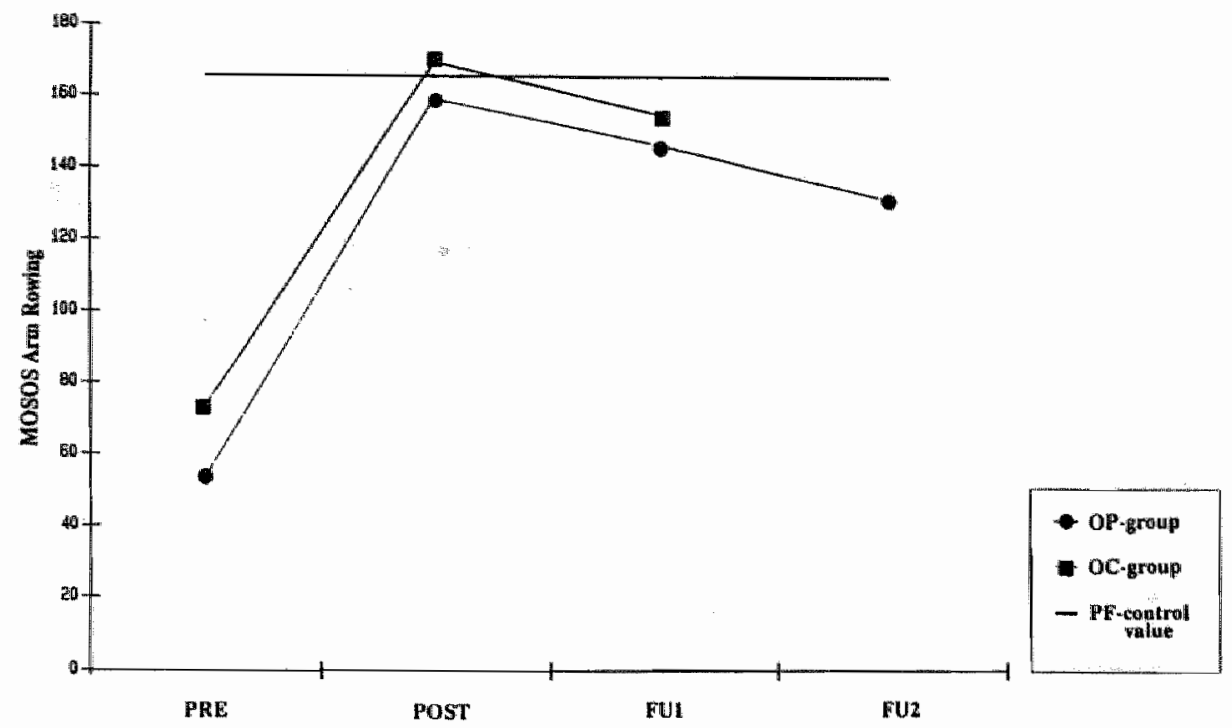

Figure 10.3 MOSOS Arm Rowing scores across treatment and follow-up.

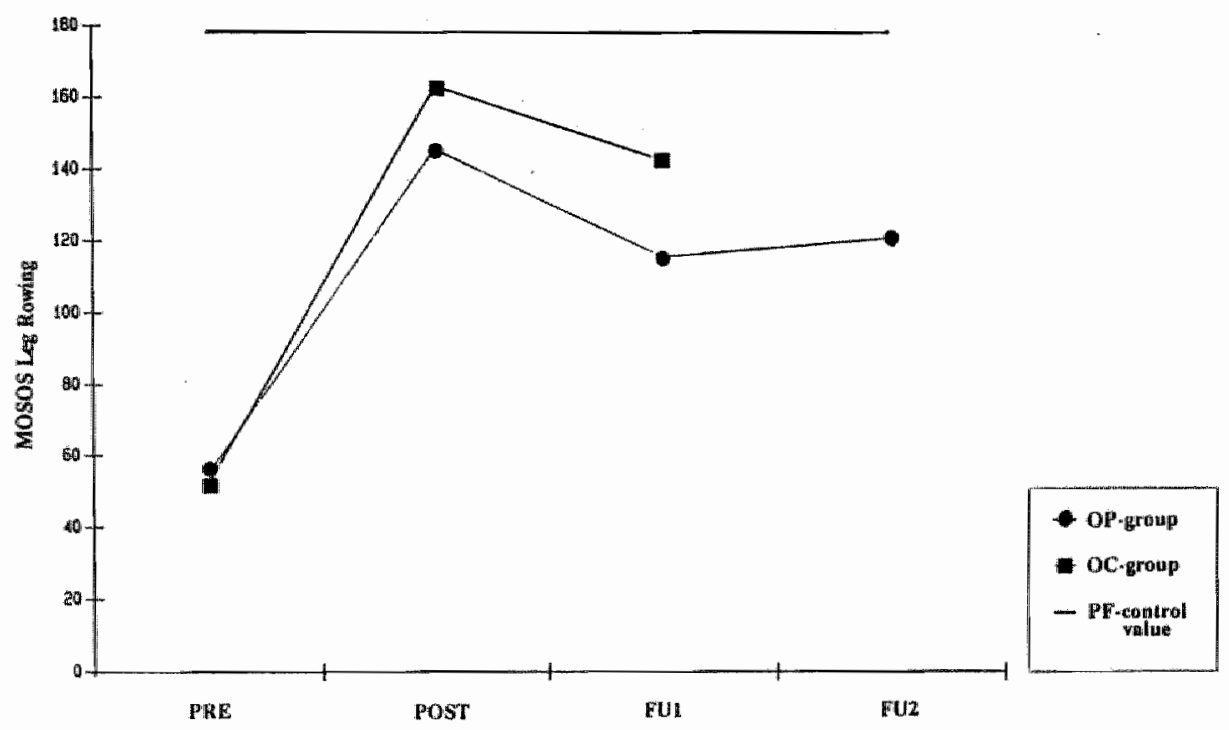

Figure 10.4 MOSOS Leg Rowing scores across treatment and follow-up. 


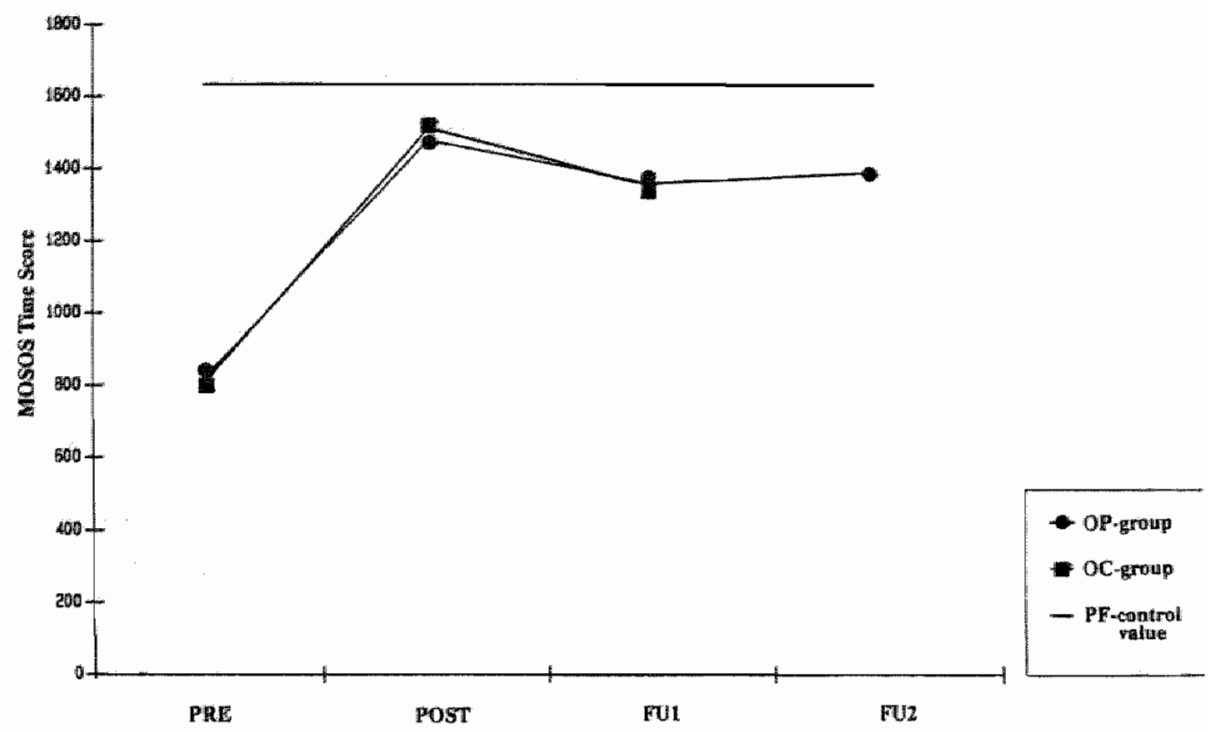

Figure 10.5 MOSOS Time scores across treatment and follow-up.

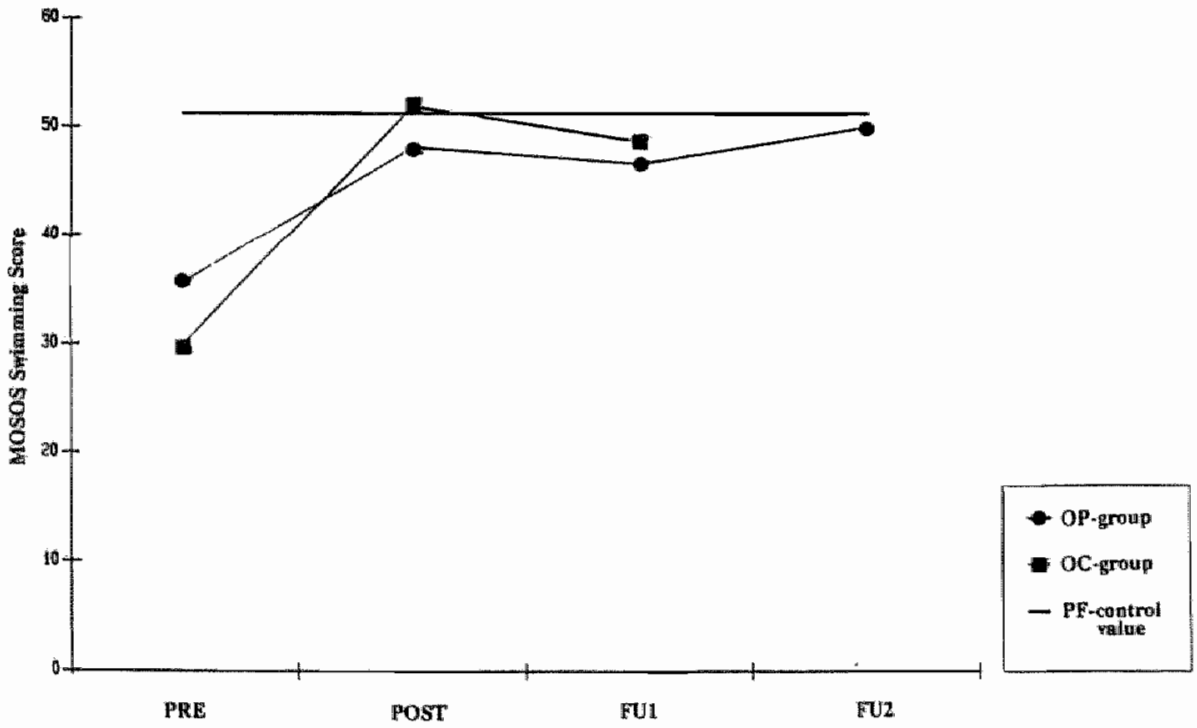

Figure 10.6 MOSOS Swimming scores across treatment and follow-up. 


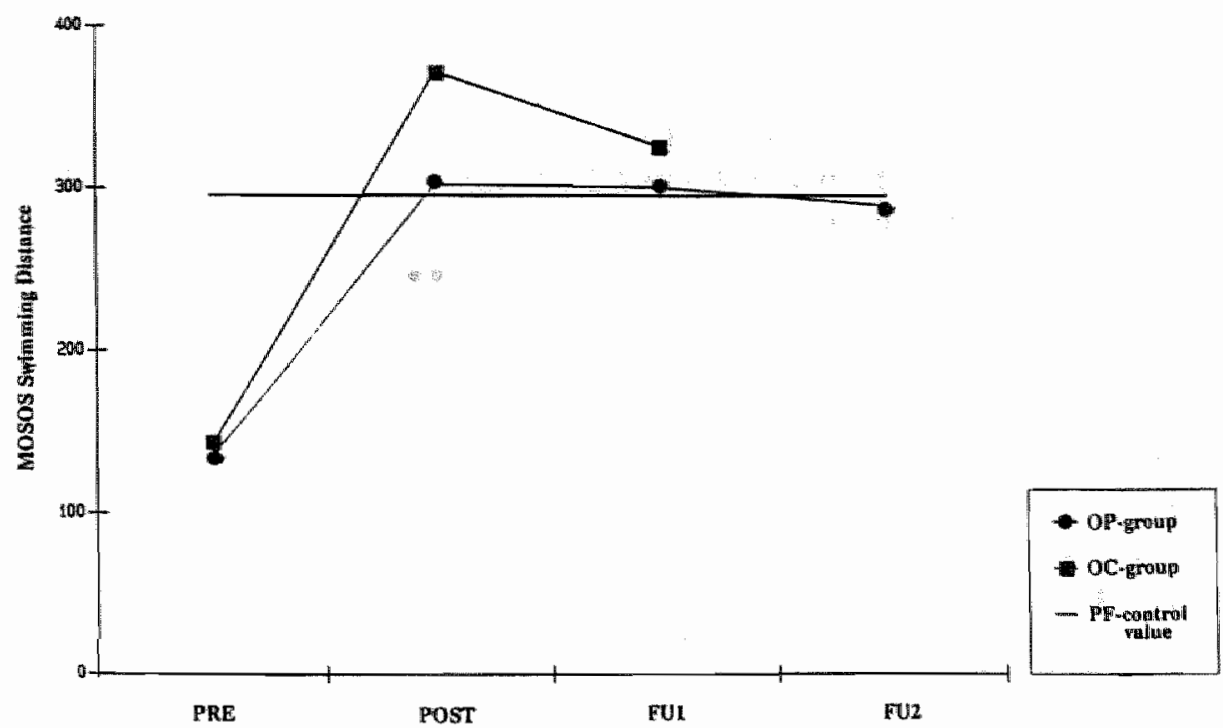

Figure 10.7 MOSOS Swimming distance scores across treatment and follow-up.

\subsection{Examination of clinically significant follow up levels}

\subsubsection{Method}

A similar method as described in the previous section is used to examine the clinical significance of the follow-up levels. For the 11 selected variables, the same criteria for clinically significant levels are applied. Patients who meet the criteria for 8 or more variables will be considered "good persisters". Patients who meet the criteria for more than 4 but less than 8 variables will be considered "moderate persisters", and patients who meet the criteria for less than 4 variables will be considerd "poor persisters". The difference in clinically significant follow-up levels between the OP and $O C$ group is examined by means of $\mathrm{CHI}^{2}$ tests. The Null Hypothesis is:

$\mathrm{Ho}_{10}$ : There is no difference between the OP and the OC groups in terms of the number of persisters.

In addition, univariate ANOVA's, with the reversed Helmert contrast for the group factor (OP, OC and PF groups) will be used to test $\mathrm{Ho}_{11}$ :

$\mathrm{Ho}_{11}$ : There is a significant difference among the experimental groups and the PF group. 


\subsection{2, Results}

The results of $\mathrm{CHI}^{2}$ test, examining the difference between the $\mathrm{OP}$ and $\mathrm{OC}$ group are summarized in Table 10.II. The mean percentage persisters is $45,5 \%$ for the OP group and 56,3\% for the OC group. More "good" persisters are found in the OC group than in the OP-group for FU1 (38.9\% vs. 10.5\%). Overall, however, $\mathrm{Ho}_{10}$ cannot be rejected: there is no difference between the groups for the three classes of persisters $\left(\mathrm{CHI}^{2}=3.128, \mathrm{p}=.209\right)$. It is expected that drop-outs are patients who did not do well, and therefore were not interested in returning to the rehabilitation center for the followup assessments. Table 10.IV., however, shows that only one drop-out was a "poor" persister. Interestingly enough, all drop-outs for the OC group at FU1 were "good" achievers. It can be concluded that the follow-up data are not positively biased by the absence of patients who were less successful.

Table 10. IV: Percentages of achievers and persisters for $O P$ and $O C$ groups. Arches indicate the origins of drop-outs.

\begin{tabular}{|c|c|c|c|c|c|c|c|c|}
\hline & \multicolumn{2}{|c|}{$\begin{array}{l}\text { Responders } \\
\text { (PRE) }\end{array}$} & \multicolumn{2}{|c|}{$\begin{array}{l}\text { Achiewers } \\
\text { (POST) }\end{array}$} & \multicolumn{2}{|c|}{$\begin{array}{l}\text { Persisters } \\
\text { (FU1) }\end{array}$} & \multicolumn{2}{|c|}{$\begin{array}{l}\text { Persisters } \\
\text { (FU2) }\end{array}$} \\
\hline OP-group: & $\mathrm{N}$ & & $\mathrm{N}$ & & $\mathbb{N}$ & & $\mathrm{N}$ & \\
\hline Good & 3 & $16 \%$ & 8 & $42 \%$ & 2 & $11 \%$ & 4 & $21 \%$ \\
\hline Moderate & 8 & $42 \%$ & 9 & $47 \%$ & 13 & $68 \%$ & 10 & $53 \%$ \\
\hline Poor & 8 & $42 \%$ & 2 & $11 \%$ & 3 & $16 \%$ & 2 & $11 \%$ \\
\hline Drop-outs & 0 & $0 \%$ & 0 & $0 \%$ & 1 & $5 \%$ & $\$ 3$ & $16 \%$ \\
\hline Total & \multicolumn{2}{|l|}{19} & \multicolumn{2}{|l|}{19} & \multicolumn{2}{|l|}{19} & \multicolumn{2}{|l|}{19} \\
\hline \multicolumn{9}{|l|}{ OC-group: } \\
\hline Good & 8 & $44 \%$ & 9 & $50 \%$ & 7 & $39 \%$ & & \\
\hline Moderate & 7 & $39 \%$ & 6 & $33 \%$ & 6 & $33 \%$ & & \\
\hline Poor & 3 & $17 \%$ & 3 & $17 \%$ & 2 & $11 \%$ & & \\
\hline Drop-outs & 0 & $0 \%$ & 0 & $0 \%$ & 3 & $17 \%$ & & \\
\hline Total & 18 & & 18 & & 18 & & & \\
\hline
\end{tabular}

ANOVA's, testing the difference between the groups (OP, OC and PF) for some of the MOSOS reveal that for the subscales Arm rowing, and the two swimming subscales no differences are found, suggesting that for these, $\mathrm{Ho}_{11}$ can be rejected: the patients did as well as the normals. Similar results are found for the OP group at FU2 (Table 10.III.). 


\subsection{Discussion}

Regardless of statistically significant changes for the 7 dependent variables described in the previous chapter, clinically significant change, post-treatment levels, and follow-up levels are examined. Seventy percent $(70 \%)$ of the subjects were classified as "moderate" to "good" responders. Eighty four percent $(84 \%)$ of the subjects were classified as "moderate" to "good" achievers. At 6-month follow up, $76 \%$ still reach "moderate" to "good" clinically significant levels. For the $\mathrm{OP}$ group, at 12 -month follow-up $74 \%$ of the patients still hold their "moderate" to "good" clinically significant levels.

When compared to an age-matched pain-free sample, no differences are found between $P F$ group mean levels and $O P+O C$ groups mean posttreatment levels for activities including arm-rowing, leg-rowing, and swimming distance. At 6-month follow-up the same results are achieved except for leg-rowing.

Generally, more $O C$ subjects reached a moderate to good level clinically. When using non-parametric statistical tests, however, these differences are not statistically significant.

In the light of the treatment-resistant nature of CLBP complaints, the conclusion seems justified that the treatment contributed in a clinically relevant way to the patients care. 



\section{Effectiveness of an operant and an operant-cognitive treatment for chronic low back pain III: broad-spectrum analysis}

\subsection{Introduction}

Several authors have reported on the different characteristics of chronic pain patients who are attending pain clinics as compared to those who are taken care of by their family physician, or who are not yet involved in the Health care system (Crook \& Tunks, 1985; Deyo, 1988). Generally, they found that pain-clinic patients score higher on measures of emotional and social dysfunctioning. Whether these problems existed before the onset the pain, or are part of the consequences of the persistent pain is a question difficult to answer. This kind of questions call for large scale prospective studies, which have not been undertaken yet. Regardless of the cause for emotional problems other than pain, it is worth examining to what extent these problems diminish as a result of behavioral management programs aimed at restoring health behaviors and functioning.

In chapter 2, the importance of 'avoidance learning' in the maintenance of CLBP has been outlined. CLBP patients may limit activity levels because of anticipated nociceptive stimuli and consequent pain. For patients, however, who are experiencing other complaints besides pain as well, avoidance also means a reduction of responsibilities and life stresses. Pain management programs, such as the ones described in Appendix A and B, are not designed to provide treatment for these non-pain complaints. Therefore, a broad-spectrum analysis is essential to identify factors that may hamper the rehabilitation process. Whenever these rehabilitation-hindering factors are identified, it is likely that an operant (OP) or operant-cognitive (OC) treatment will only result in short-term improvements, or in no improvement at all. 
In the two previous chapters, dependent variables were chosen according to the three-systems model of chronic pain, and can be situated within the functional analysis of CLBP. In addition, a broad spectrum analysis places the chronic pain problem in the broader context of problem areas other tham pain. Such an analysis aims to quantify rehabilitation-hindering and -promoting factors. In this study, only variables related to self-reported complaints other than pain are included.

In this chapter, a study is described which is an extension of the studies described in the 2 previous chapters, and attempts to answer three questions:

1. From a broad-spectrum analysis, what are the characteristics of the CLBP patients who have participated in the OP and OC treatment. This analysis will be carried out in terms of clinically significant scores on indices tapping self-statements of emotional problem areas other than pain.

2. If any problem areas are identified, do these change as a result of the treatment?

3. Does a relationship exist between the existence of these problem areas and treatment outcome in terms of the pain measures?

\subsection{Method}

\subsubsection{Measures:}

Table 11.I. shows the list of questionnaires that have been used in the study. Personality questionnaires have been completed only before treatment. For the MMPI, American norms are utilized. The items of the NPV are the translated items of the California Psychological Inventory (Gough, 1964), which have been modified and completed with new items which are more relevant to the Dutch population. The NVM is a Dutch personality questionnaire that is based on the translated items of the MMPI. Factoranalysis revealed different scales than the original MMPI. The PMT is a questionnaire that is designed to measure achievement motivation, debilitating anxiety, and fascilitating anxiety. These constructs, wich are based on the work of Atkinson (e.g. Atkinson \& Feather, 1966) and Alpert \& Haber (1960), are thought to be relatively stable personality characteristics. Depending on situational factors, they can lead to a functional or dysfunctional achievement level.

For these personality measures, scores with a corresponding T-score of at least 70 were considered clinically significant. 
At the PRE- and POST treatment assessment phase, and at the 6-month and 12-month follow-up assessment phase, the $O P$ and $O C$ group completed the BDI, SIG, FSS and MOCI. For purely practical reasons, the WLCgroup only completed the questionnaires indicated with an asterisk before and after their 6 measurement points. As a measure of depression, the BDI was chosen as it was shown by Love (1987) to be the most efficient instrument for identifying depression in CLBP patients. The revised 21 item version of the BDI was used, for which the cut-off score of 17 was considered. The Dutch version was the one suggested by a Dutch commission for the Standardization of Depression Questionnaires (Zitman et al., 1989). The SIG is a questionnaire aimed at quantifying aspects of social behavior, including the tension experienced and the frequency of socially adequate behavior. Four interpersonal behaviors are considered: expression of negative emotions, expression of uncertainty and inadequacy, assertion, and expression of positive feelings. Only the total scores are used in our study. A Dutch version of the FSS-III (Arrindell \& Zwaan, 1982) and the MOCI (Sternberger \& Burns, 1990; Kraaykamp et al., 1990) are included. For the Dutch population, the MOCI total score and the "checking" and "cleaning" subscales are sufficiently reliable. The subscale for "doubting" has a limited reliability, and the subscale "slowness" does not meet psychometric criteria (Kraaykamp et al., 1990). Therefore, the latter two are excluded from the study.

For these measures, appropriate norms and cut-off scores, reported in the aforementioned papers, are utilized to determine clinical significance.

\subsubsection{Statistical analyses}

In order to answer question 1, measures were clustered on the basis of clinically significant scores. A binary matrix is constructed for patients and problem areas indicating clinically significant problem areas at pre-treatment assessment for each subject ( $1=$ clinically significant score, $0=$ not clinically significant score). Problem areas are measured with the scales of the above-mentioned questionnaires. The resulting 37 (subjects) $\times 38$ (problem areas) matrix is subsequently subjected to a Hierarchical Classes Analysis (HICLAS) as described by De Boeck and Rosenberg (1988). HICLAS provides a discrete, categorical representation of objects (in our case subjects) and attributes (in our case problem areas) simultaneously. A novel feature of HICLAS is that it postulates an order relation among the subject classes and among the problem area classes. In each subject class, subjects with similar problem areas are clustered, and in each attribute class, problem areas coinciding frequently are clustered. The analysis results in a simultaneous graphical representation of the classes. 


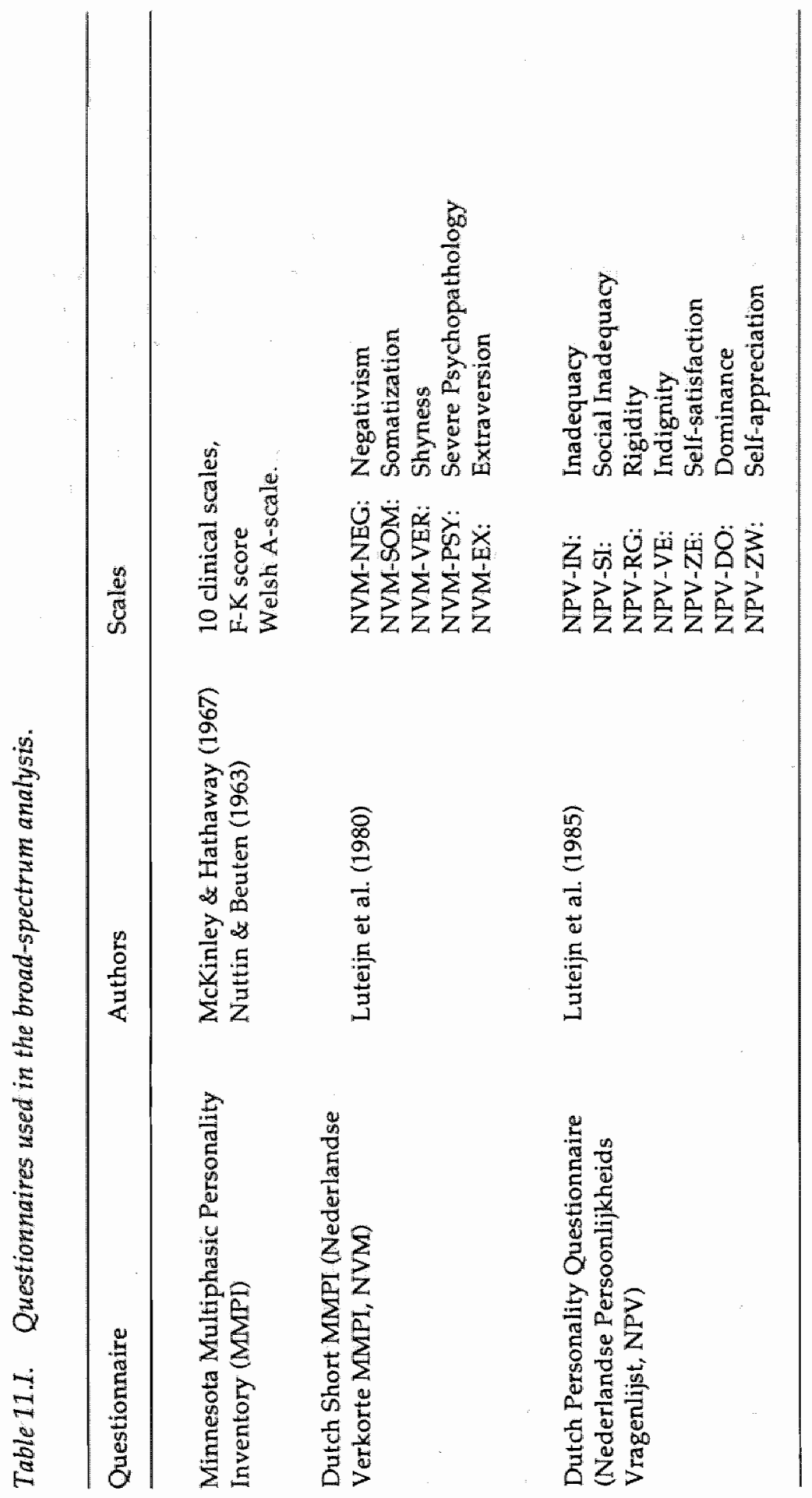




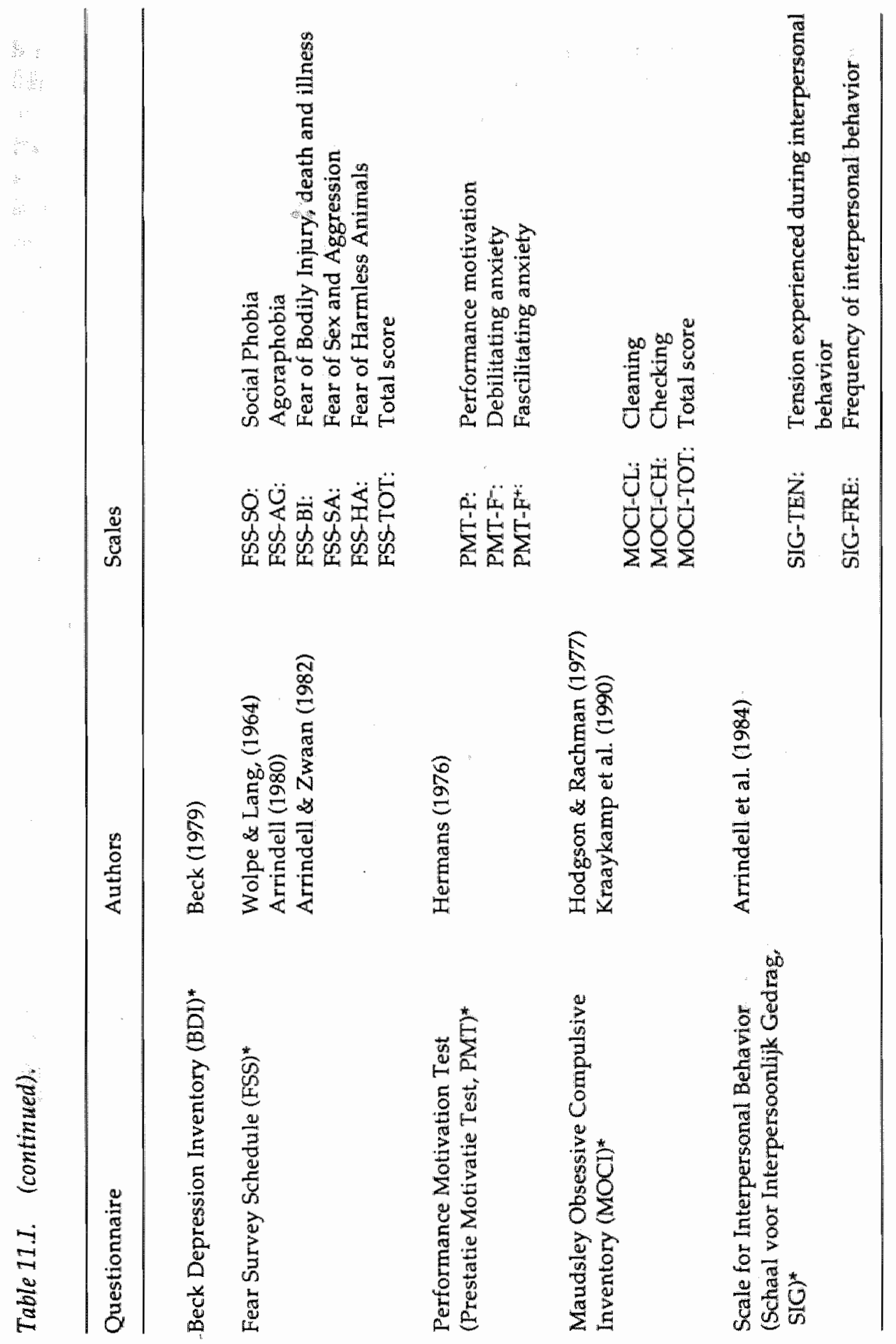


It summarizes the equivalence and order relations among the classes and the way subject classes and problem area classes are associated to each other. For each subject, the goodness of fit by which the subject belongs to that class is calculated. This goodness of fit calculation is also performed for the problem areas, and an overall goodness of fit calculation is made as well. Only measures that are found in the problem area classes with a goodness of fit of at least .45 will be considered in further statistical analyses.

In order to answer question 2 , three doubly multivariate repeatedmeasures ANOVA's are performed, during which all dependent variables are considered simultaneously in a Variable factor. For these MANOVA's the significance level is set at .05. Besides the Variable factor, a Group factor and a Time factor are included. In the first MANOVA, the Group factor consists of the two experimental groups (OP and $O C$ ) and the repeated measures factor is Time (PRE, POST). The Null Hypotheses are:

$\mathrm{Ho}_{12}$ : The dependent variable will not change across PRE and POST assessment , and

$\mathrm{Ho}_{13}$ : This will be the case for both groups.

For the second MANOVA, the three groups, including the WLC, are considered. The Variable factor consisted of the variables for which scores of the WLC-group are available. In this analysis, the Null Hypothesis is:

$\mathrm{Ho}_{14}$ : The change across PRE - POST assessment phases is equal for all three groups.

Reversed Helmert contrasts are used for the Group factor, the first contrast comparing both experimental groups with the WLC-group, the second contrast comparing OP-group with OC-group.

If the previous MANOVA's reveal any Group $X$ Time and/or Time effect, a third and a fourth MANOVA is to be carried out. In the third analysis, only the experimental groups are included in the Group factor, and the Time factor now includes the first follow-up measurement (POST, FU1). Here, the Null Hypotheses are:

$\mathrm{Ho}_{15}$ : There is no difference among POST - FU1 assessment phases, and $\mathrm{Ho}_{16}$ : This will be the case for both groups.

The fourth MANOVA only deals with the OP group and makes a comparison among POST and FU2. At the time when the analyses were made, FU2 
measurements were not finished for the OC-group. The Null Hypothesis here is:

$\mathrm{Ho}_{17}$ : There will be no significant difference between POST and FU2.

In order to answer question 3, Cramers $V$ will be calculated to test the association between classes of problem areas and classes of responders, achievers, and persisters respectively. The Hypothesis is:

$\mathrm{Ho}_{18}$ : There is no association among classes of responders, achievers, and persisters.

In addition, ANOVA for repeated measures will be performed for the variable "Health behavior $A$ " (see chapter 9), for which a Class factor consists of the classes of subjects with similar problem areas. The variable "Health behavior $\mathrm{A}^{\prime \prime}$ is chosen because it does correspond with the component with the largest percentage explained variance. Table 9.IX. also revealed no Group effect and no Group $x$ Time effect for both treatment groups on this variable. This provides the opportunity to analyze the data without considering group differences. ANOVA is carried out tot test the Null Hypothesis:

$\mathrm{Ho}_{19}:$ The difference among the assessment phases is equal for the three classes of subjects.

\subsection{Results}

\subsubsection{Broad-spectrum analysis: clinical picture of the sample}

Figure 11.1. shows the results of HICLAS performed on the binary subjects $X$ problem areas matrix. Six classes of problem areas (with all possible bundles except bundle [100]), and 4 classes of subjects are found (with bundles [100], [010], [001] and [101]). For this classification, 23 (60\%) problem areas are relevant, of which only $18(47 \%)$ reach a goodness-of-fit of at least 0.45 . Fifteen of the 38 problem measures cannot be used to classify the population according to clinically significant problem areas, and constitute an undefined class of problem areas (with bundle [000]). An undefined class of subjects (also with bundle [000]) consists of 9 patients (24\%). These patients report a scattered pattern of problem areas that differs from the patterns found in the other classes of subjects. Table 11.II. shows the characteristic problem areas for the classes of patients. Based on the respective 
values of goodness-of-fit of the problem areas, the classes of subjects can be labeled as follows:

\section{Class 1: Phobics \\ Class 2: Severely distressed \\ Class 3: Moderately distressed \\ Class 4: Moderately distressed phobics}

As presented in figure 11.1, subjects of the four classes generally have the following characteristics in common: They report somatic concerns (MMPI$\mathrm{Hs}$ ) and tend to claim the attention of others for these concerns (MMPIHy). Of interest is that all subjects report a high level of achievement motivation (PMT-P): They try to excel in challenging and task-oriented situations. Furthermore, they are future-oriented, ambitious and perseverant. In addition to these general characteristics, different classes of subjects have the following characteristics:

Class 1: These subjects can be described as fearful. They fear not being able to cope adequately in complex and new situations (PMT-F-). Furthermore, they fear social interactions, body injury, sex and agression, harmless animals, and they suffer from agoraphobia (FSS scales).

Class 2: These patients have elevated scores on all measures except on PMT-F+ and MMPI-F-K. They have similar complaints as those in class 1 , but in addition, they are depressed (BDI, MMPI-D) and experience feelings of inadequacy (NPV-IN) and loose thinking (MMPI-Sc). They tend to distrust and criticize other people (NPVVE, NVM-NEG), are tense in social interactions (SIG-TEN, FSSSO), and show obsessive-compulsive behavior (MOCI). Patients with similar test scores often report multiple somatic complaints (NVM-SOM).

Class 3: In contrast to patients of the previous class, patients of class 3 have low scores on most of the scales, except for measures of depression (MMPI-D). They also report being able to cope relatively well in complex and new situations (PMT-F+).

Class 4: These subjects share the characteristics of class 1 and class 3 . They complain of multiple fears, are depressed, but report to cope relatively well in complex and new situations. 


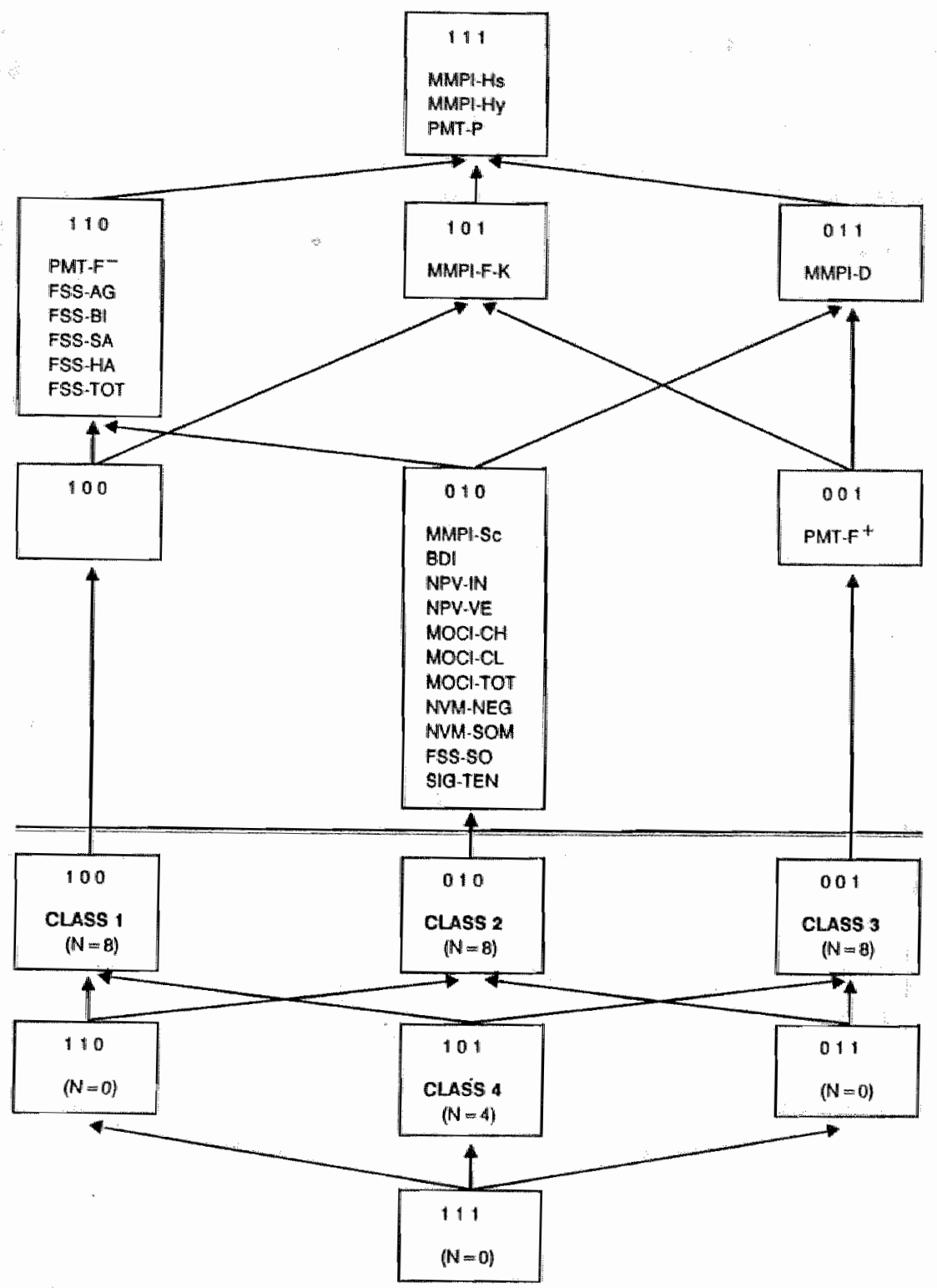

Figure 11.1. Graphical representation of HCLAS solution with hierarchical classes of subjects and problem areas simultaneously. The hicrarchical structure is indicated by bundles $[100]_{1}[010]$, etc.. belonging to one of the three levels, and by arches specifying which classes of a lower level belong to classes of a higher level. For example, subjects belonging to class 3 with bundle l001 J are not only characterized by problem area class with the corresponding bundle [001], but also by classes with bundles [101] and 1011], and finally by problem area class with bundle [111]. Subject class 4 with bundle [101] is situated at a higher level and has characteristics of both subject class 1 with bundle [100] and subject class 3 with bundle [001]. Undefined classes are indicated by bundle [0001 and are not shown in this figure. 
Table 11.II. HICLAS classes for subjects and mean raw scores for the corresponding problem areas. Frames refer to the attributes belonging to the respective subject classes.

\begin{tabular}{|c|c|c|c|c|c|}
\hline \multirow[b]{2}{*}{ Attributes } & \multirow[b]{2}{*}{$\begin{array}{l}\text { Goodness } \\
\text { of fit }\end{array}$} & \multicolumn{4}{|c|}{ HICLAS Classes } \\
\hline & & $\begin{array}{l}\text { Class } 1 \\
(100) \\
N=8\end{array}$ & $\begin{array}{l}\text { Class } 2 \\
(010) \\
N=8\end{array}$ & $\begin{array}{l}\text { Class } 3 \\
(001) \\
N=8\end{array}$ & $\begin{array}{l}\text { Class } 4 \\
(101) \\
N=4\end{array}$ \\
\hline MMPI-Hs & .83 & 16.9 & 25.0 & 18.9 & 17.2 \\
\hline MMPL-Hy & .87 & 35.0 & 36.7 & 37.1 & 34.0 \\
\hline MMPL-F-K & .68 & -16.0 & -2.1 & -12.0 & -12.0 \\
\hline MMPI-D & .70 & 24.9 & 31.2 & 26.2 & 29.7 \\
\hline MMPI-SC* & .42 & 11.5 & 30.6 & 13.6 & 18.7 \\
\hline $\mathrm{PMT}-\mathrm{P}$ & .59 & 20.2 & 21.5 & 11.6 & 17.7 \\
\hline PMI-F- & .68 & 13.6 & 16.9 & 6.1 & 12.0 \\
\hline PMT-F+ & .47 & 8.4 & 4.1 & 10.2 & 9.7 \\
\hline FSS-SO* & .35 & 26.2 & 30.7 & 16.5 & 23.5 \\
\hline FSS-AG & .70 & 23.9 & 29.4 & 14.7 & 17.5 \\
\hline FSS-BI & .75 & 30.9 & 31.1 & 15.2 & 23.5 \\
\hline FSS-SA & .73 & 15.1 & 18.5 & 9.5 & 13.0 \\
\hline FSS-HA & .69 & 16.1 & 17.1 & 7.7 & 11.5 \\
\hline FSS-TOT & .70 & 188.7 & 220.6 & 110.7 & 148.5 \\
\hline NPV-IN & .64 & 9.1 & 27.2 & 12.0 & 15.0 \\
\hline NPV-VE* & .38 & 17.4 & 24.9 & 14.5 & 17.5 \\
\hline NVM-NEG & .38 & 10.6 & 21.7 & 13.6 & 16.7 \\
\hline NVM-SOM & .54 & 14.9 & 27.0 & 18.2 & 15.5 \\
\hline $\mathrm{MOCl}-\mathrm{CH}$ & .55 & 1.1 & 5.1 & 1.5 & 2.7 \\
\hline MOCI-CL* & .38 & 1.1 & 3.2 & 2.0 & 2.5 \\
\hline MOCI-TOT & .50 & 3.6 & 12.5 & 5.2 & 8.5 \\
\hline SIG-TEN & .46 & 110.0 & 136.9 & 76.9 & 108.7 \\
\hline BDI & .54 & 9.1 & 21.5 & 9.0 & 17.5 \\
\hline
\end{tabular}

* Measures which are omitted in the statistical analyses because their goodness-of fit $<0.45$.

These descriptions should be considered with caution. When examining test-taking attitude, only subjects of class 2 have responded to the tests in an open and frank way according to their MMPI F-K scores. Subjects in the three other classes have made an attempt to put themselves in a favourable light, and might have underreported their complaints.

On the assumption that these scores are not overly distorted by social desirability, class 1 and class 4 can be grouped together as a group of phobic patients (new class 1). Nine patients belong to the undefined class 
of subjects. Inspection of their patterns of clinically significant scores, 3 patients show severe pathology and can be added to class 2 (new class 2). The other 6 subjects show very little pathology and can be added to class 3 (new class 3 ). There is no association found between these HICLAS (Phobics, Severely distressed, Moderately distressed) classes and the treatment groups (OP and $\mathrm{OC}$ ) $\left(\mathrm{CHI}^{2}=3.58, \mathrm{p}=.167\right)$, suggesting that the different problem areas are equally divided over the experimental groups.

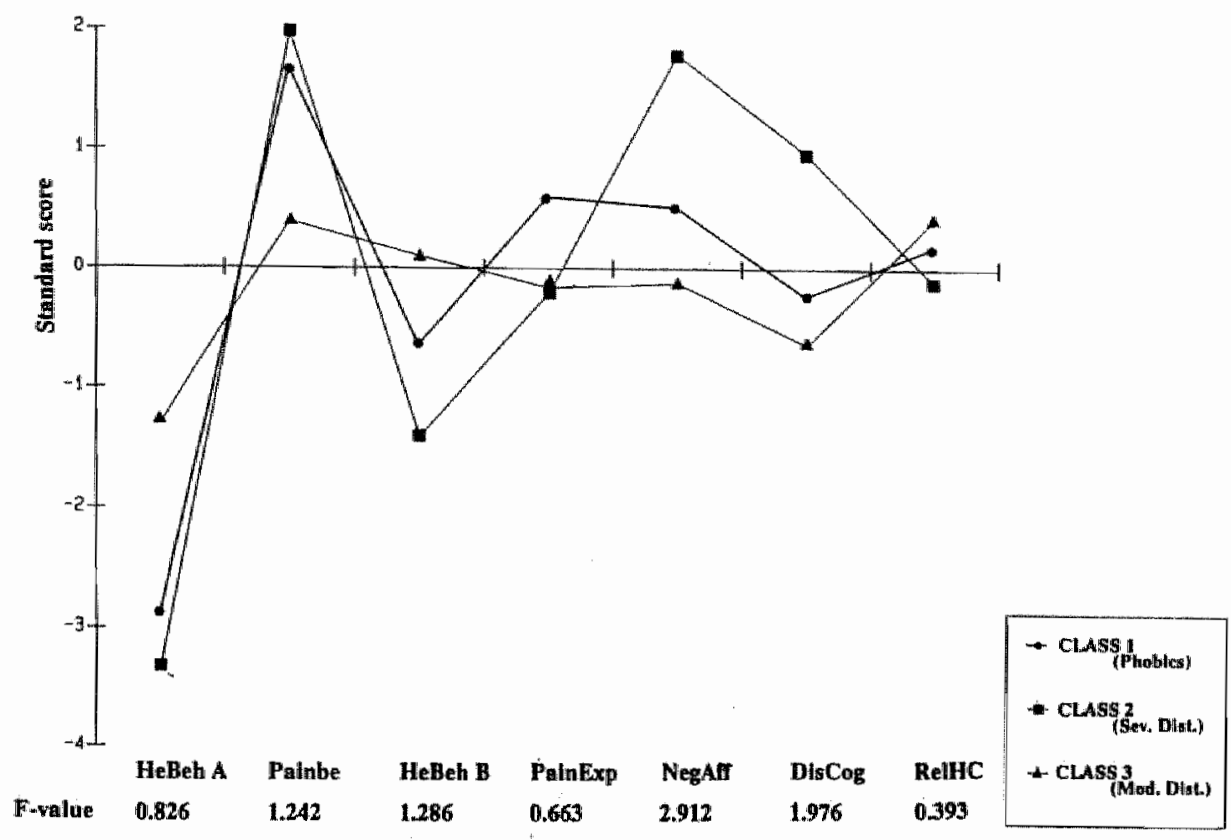

Figure 11.2: Mean PRE-assessment scores on the 7 components for the three classes of subjects. F-values refer to the MANOVA testing differences among the classes of subjects.

Fig. 11.2 shows the mean pre-assessment scores on the 7 components for the three classes of subjects. Visual inspection suggests interesting differences among the three classes of subjects. A MANOVA testing these differences, however, revealed no significant class effect (Wilks Lambda= $.625, \mathrm{~F}=1.02, \mathrm{p}=.448$ ). F-values of the separate ANOVA's are presented in figure 11.2.

These data suggest that before the start of treatment, there is no significant relation between the functional analysis and the broad spectrum analysis. In other words, the extent to which complaints other than pain are present 
tells us nothing about the level of pain-related measures. The broad-spectrum analysis provides new information that cannot be derived from the functional analysis.

The HICLAS classification was defined by 18 variables. Because of the high intercorrelation among the FSS subscales (Arrindell, 1980), only FSSBI, which has the best goodness-of-fit, is selected for further analyses. From the MOCI, only MOCI-CH, which also has the best goodness-of fit is selected in the further analyses. The remaining 13 scales, can be divided in two groups:

$\begin{array}{ll}\text { personality variables: } & \text { MMPI-Hs } \\ & \text { MMPI-Hy } \\ & \text { MMPI-D } \\ & \text { MMPI-F-K } \\ & \text { NPV-IN } \\ & \text { NVM-SOM } \\ & \text { PMT-P } \\ & \text { PMT-F- } \\ & \text { PMT-F+ } \\ & \\ \text { other variables: } & \text { FSS-BI } \\ & \text { MOCI-CH } \\ & \text { SIG-TEN } \\ & \text { BDI }\end{array}$

The first group of personality variables are considered more stable traits, that are less likely to change over time. The second group is more likely to change. For this second group of variables scores are available at the subsequent assessment phases, stich that they can be included in the multivariate analyses.

11.3.2 Immediate treatment effects: Comparison between the $O P$ and $O C$ groups. The first MANOVA, performed for the two treatment groups, revealed a Time effect but no Group $X$ Time effect (Table 11.III). These results suggest that $\mathrm{Ho}_{12}$ cannot be rejected: in general, the experimental groups do not differ. $\mathrm{Ho}_{13}$ should be rejected: there is a significant decrease over time, but this change is the same for both groups. Table 11.IV shows the univariate ANOVA's for the variables separately. As can be seen, only the variable BDI is responsible for the Time effect. 
Table 11.III. F-values of Doubly Multivariate repeated measures ANOVA for the variables FSS-BI, BDI, SIG-TEN, and MOCI-CH. I: Comparison between PRE and POST assessment. II: Comparison between POST and FU1 assessment. III: Comparison between POST and FU2 assessment for the OP group only.

\begin{tabular}{lccc}
\hline Effect & I & II & III \\
\hline Group & 0.04 & 1.10 & \\
Variable & $60.63^{* * *}$ & $41.20^{* * *}$ & $39.03^{* * *}$ \\
Time & $5.36^{*}$ & 0.00 & 0.16 \\
& & & \\
Group $\times$ Variable & 1.46 & 1.30 & \\
Group x Time & 0.27 & 0.45 & 0.35 \\
Variable X Time & 1.37 & 0.29 & \\
Group x Variable X Time & 0.45 & 0.58 & \\
\hline
\end{tabular}

${ }^{*} \mathrm{p}<.05,{ }^{* *} \mathrm{p}<.01,{ }^{* * *} \mathrm{p}<.001$.

11.3.3. Immediate treatment effects: Comparisons among the three groups under study.

An analysis similar to that performed for the two experimental groups was also performed for the three experimental groups. MANOVA revealed only a Time effect, but no Group X Time effect for any of the contrasts (Table 11.V.). This means that the $\mathrm{Ho}_{14}$ cannot be rejected: The WLC-group scores do not change differently over time than the treatment groups. It is very likely that for the variables under study, the change over time is not due to the treatment. There is no reason to believe that a change over time for the other variables would be due to the treatment. Conclusions about possible Time effects as shown in the univariate ANOVA's should be made with caution.

11.3.4. Long-term treatment effects: 6-months and 12-months follow-up.

Table 11.III, showing the results of the MANOVA testing the difference between POST and FU1, reveals no Time effect nor a Group X Time effect. Similar results are obtained at 12 -months follow-up for the OP group. $\mathrm{Ho}_{15}$ $\mathrm{Ho}_{16}$ and $\mathrm{Ho}_{17}$ cannot be rejected: the results suggest that no significant changes occured after the post-assessment phase. 


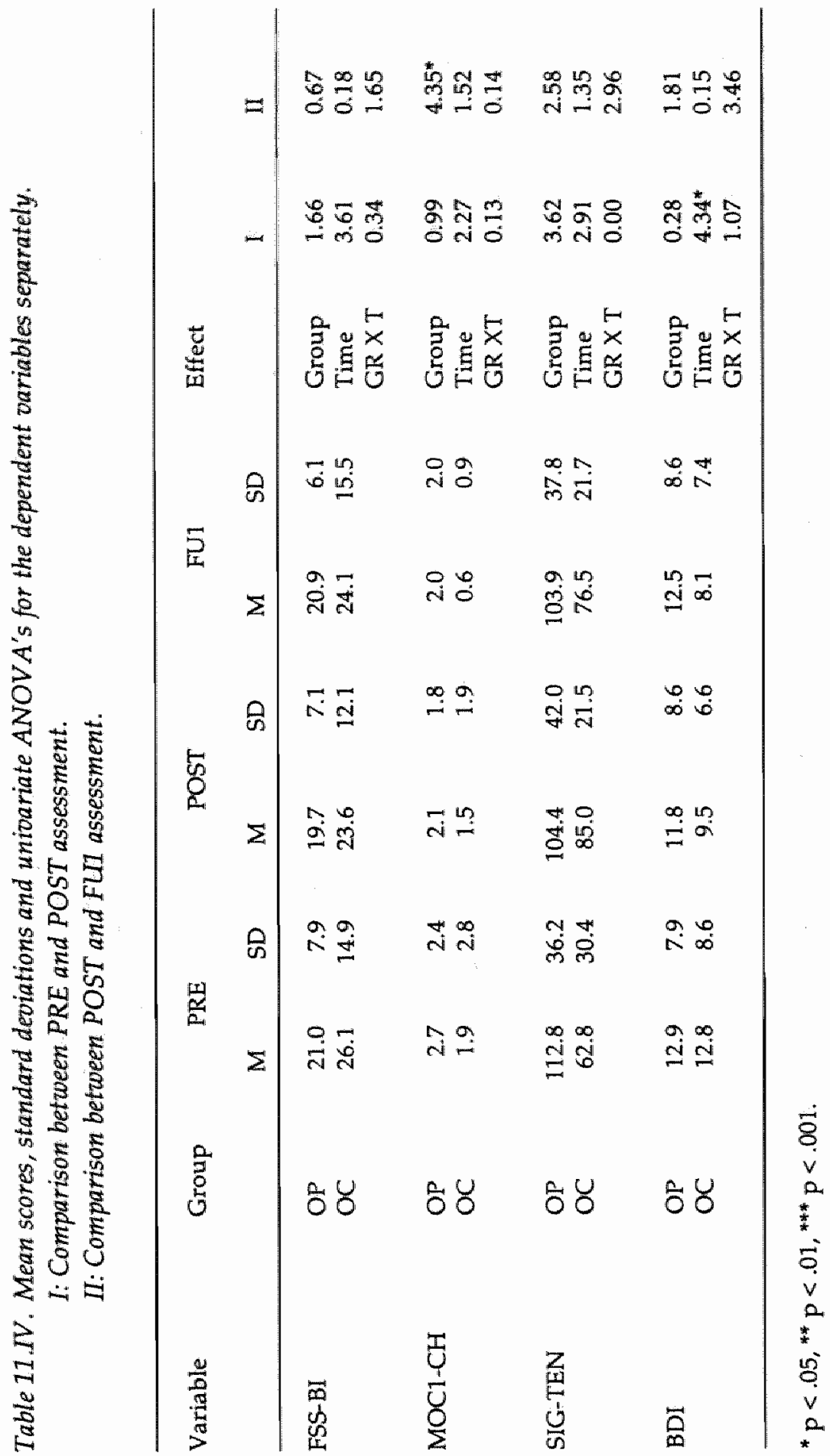


Table 11.V. Doubly Multivariate repeated-measures ANOVA for the variables FSS-BI, MOCI-CH, SIG-TEN, and BDI simultaneously for the three groups (OP, OC and WLC). Comparison between the three groups with Reversed Helmert contrasts.

\begin{tabular}{lc}
\hline Effects & Approx. F. \\
\hline Group & \\
1st Rev. Helmert (1,2 vs 3) & 0.04 \\
Variable & $87.25^{* * *}$ \\
Time & $5.34^{*}$ \\
Group X Variable & \\
1st Rev. Helmert (1,2 vs 3) & 1.73 \\
Group X Time & \\
1st Rev. Helmert (1,2 vs 3) & 0.32 \\
Variable X Time & 1.46 \\
Group X Variable X Time & 0.14 \\
1st Rev. Helmert (1,2 vs 3) & \\
\hline
\end{tabular}

${ }^{*} \mathrm{p}<.05,{ }^{* *} \mathrm{p}<.01,{ }^{* * *} \mathrm{p}<.001$

11.3.5. Relationship among problem areas and classes of clinically significant change, and post-treatment level.

No significant relationship is found between classes of 'responders', 'achievers', 'persisters (FU1)' and classes of problem areas. Cramers' $\mathrm{V}$ is $.22\left(\mathrm{CHI}^{2}=3.73, \mathrm{p}=.443\right), .30\left(\mathrm{CHI}^{2}=6.68, \mathrm{p}=.154\right)$, and $.28\left(\mathrm{CHI}^{2}=2.484, \mathrm{p}=.289\right)$ respectively. Consequently, $\mathrm{Ho}_{18}$ cannot be rejected.

\subsubsection{Relationship among problem areas and change in health behavior.}

Figure 11,3. shows the graphical representation of the change of variable

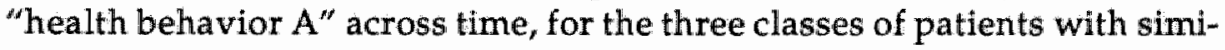
lar problem areas. Visual inspection suggests similar initial levels of health behavior for the three groups, but a divergence across time. As expected, class 2 shows the least improvement. Results of MANOVA are confirmative. In accordance with the findings in chapter 9, a significant Time effect is found (Wilks Lambda $=.340, \mathrm{~F}=2.731, \mathrm{p}=.000$ ). In addition a Time $X$ Class interaction effect is found (Wilks Lambda $=.730, \mathrm{~F}=2.731, \mathrm{p}=.037$ ). $\mathrm{Ho}_{19}$ must be rejected: subjects who report other complaints besides pain do worse than the subjects who are phobic or who are only moderately distressed. 


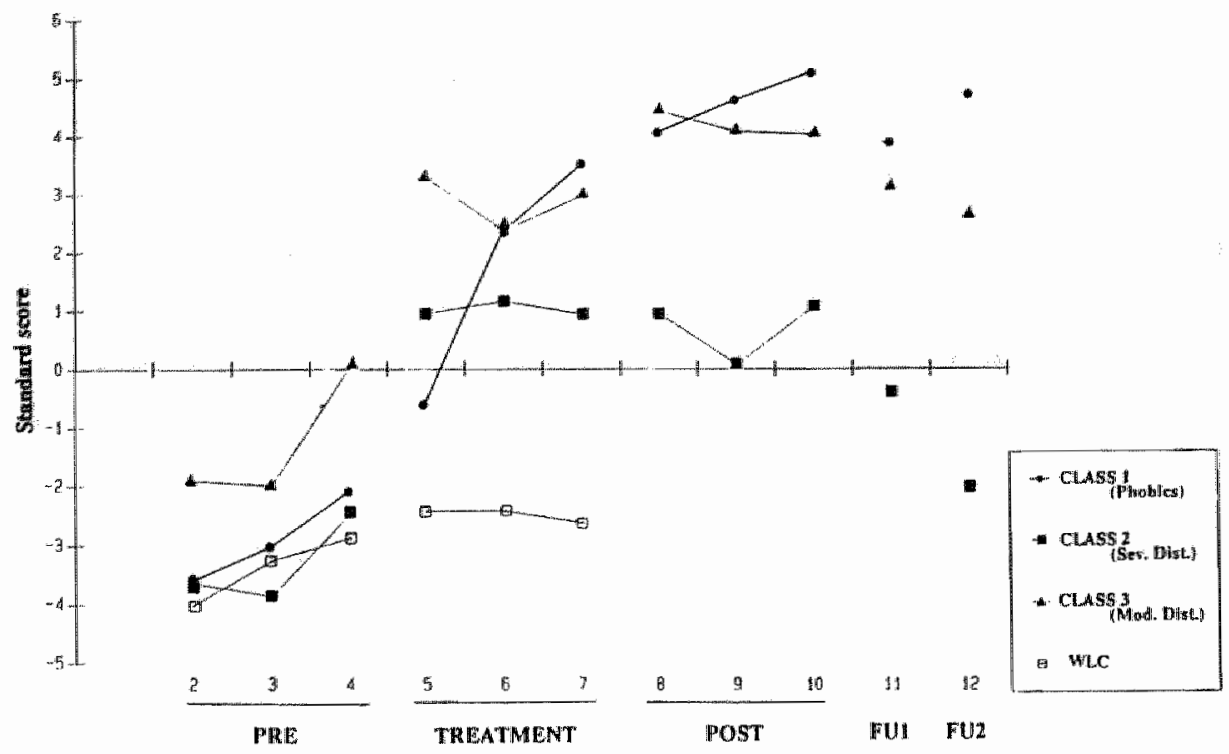

Figure 11.3. Graphical representation of component "Health Behavior $A^{\prime \prime}$ across Time, for the three classes of subjects.

\subsection{Discussion}

This chapter dealt with the broad-spectrum analysis of the CLBP sample of our therapy outcome study. Characteristic problem areas other than pain were derived empirically by means of HICLAS. In this study, 8 different questionnaires were completed by the subjects. From the 38 measures, only 23 measures were selected for the definition of classes of patients with similar problem areas. In accordance with the vast literature on MMPIprofiles in chronic pain, all patients scored high on the MMPI HS and Hy scales, suggesting somatic concerns and a tendency to claim the attention of others to these concerns. A rather novel finding is that the majority of our subjects report a high level of achievement motivation, suggesting a continuous pursuit of success and perfection. It is very likely that these subjects find themselves in a conflict situation as the chronic pain disability takes away the possibilities to achieve success. The consequent tension may increase the patients suffering and experienced pain.

Quite striking, and rather unexpected, 54\% of the subjects were phobics (as measured with FSS), with fear of body injury being the most predominant feature. The relation between CLBP and anxiety has received rela- 
tively little attention in the literature. The coexistence between CLBP and anxiety can be explained in at least two ways: from a respondent conditioning, and from an operant conditioning perspective. From the respondent perspective, pain is the result of continuous muscle tension occurring under personally relevant stressful events (Flor et al., 1984). Anxious people are likely to have an increased physiological arousal level, which can; in the long run, lead to muscle spasm associated with pain. The presence of certain cues, which have the potential to announce the occurrence of a fearful event, might increase or trigger muscle tension as an anticipatory reaction.

From the operant perspective, motoric deficits can be negatively reinforced when they have the potential to avoid the fear-provoking stimuli. A CLBP with a dog phobia may learn that resting and staying at home also means a successful avoidance from fearful streets where dogs dwell.

Although a slight improvement in these measures is found across time, no statistical significance is reached when the scores of the experimental groups are compared with the WLC group. This means that, as expected, both the OP and OC treatment did not have any impact on the problem areas other than pain.

Furthermore, no relationship was found between the kind of pathology besides pain, and clinically significant improvement levels, post-treatment levels, and follow-up levels. The most important finding, however, is the significant association between the presence of complaints other than pain, and the level of reduction in avoidance behavior during treatment: patients who report several other complaints besides fear and pain don't change their avoidance behavior as much as the phobic and less severely distressed patients. Even worse is that at follow-up, their levels of health behavior have decreased considerably. This in accordance with the assumption that other psychological complaints may hamper the rehabilitation process.

Unexpectedly, however, the phobic subjects (class 1) do as well as the moderately distressed but non-phobic patients (class 3 ). The question can be raised whether the effectiveness of the operant conditioning program is not based on exposure rather than the process of applying reinforcement to quota achievement. Exposure is a behavior therapy technique, successfully being applied to phobias, during which the client is systematically exposed to the feared object under the condition of response prevention. This technique allows habituation to the feared object, and enables the experience that the anticipated unpleasant or noxious event does not take place. In our case, anticipated pain and fear of injury might have been reduced by repeatedly exposing patients to exercise tasks small enough to be mastered. 
The actual difficulty of meeting quotas varies across treatment since quotas are first started below initial levels of tolerance. Although the FSS-BI (fear of body injury) scale has not been developed for CLBP patients, an almost significant decrease is seen as a result of treatment.

Dolce et al. (1986) found that exercise quota systems not only increased activity levels, but also self-efficacy expectations. The exposure hypothesis would also be in line with the fear-avoidance model of pain (Lethem et al., 1983) of which the central concept is fear of pain and consequent avoidance. Chronic pain patients, who are fearful of pain, choose an avoidance strategy and thereby risk a number of physiological and psychological consequences which promote the development of the invalid status. In order to falsify this hypothesis, a simplified treatment in which exposure would be the main ingredient, and in which the contingency management techniques are omitted, should be compared with the operant program as provided in this study. Such a simplified approach, however, is not likely to work for patients who are highly disabled motorically, for whom shaping procedures (see chapter 8 ) still might be necessary. In addition, the approach will not work for phobic patients who also have other complaints (similar to those of class 2). 


\section{CHAPTER 12}

\section{Desynchrony following operant and operant-cognitive treatment of chronic low back pain}

\subsection{Introduction}

In chapter 2, the three response systems of chronic pain were described and an attempt was made to examine their interrelationships. Based on Lang's (1968) caution about emotional responses as loosely related systems, Rachman \& Hodgson (1974) introduced the concept of discordance and desynchrony in fear and anxiety. Discordance refers to the low degree of correlation between 2 or more response systems, and desynchrony refers to the low degree of covariance as response systems change during treatment. Rachman and Hodgson hypothesized that synchrony would be associated with better treatment outcome. This hypothesis is confirmed by Vermilyea et al (1984) who empirically examined the predictive value of desynchrony in the behavioral treatment of agoraphobia. They found that non-responders to treatment were more likely to be 'desynchronous' subjects.

The phenomena of discordance and desynchrony have been examined mostly in phobic patients (e.g. Barlow et al., 1980; Liddell et al., 1987; Gerew et al., 1989). One study examined the role of discordance in psychophysiological disorders (including headaches, insomnia, and sexual dysfunction; Gannon \& Haynes, 1986) and two studies have demonstrated desynchrony during the behavioral treatment of chronic pain (Phillips, 1980; Kincey \& Benjamin, 1984). Philips (1980) has demonstrated that desynchrony occurs between reported pain intensity levels and pain behavior in chronic headache patients. Kincey \& Benjamin (1984) examined the relationship of measures of self-reported pain experience, negative mood state and activity levels of 12 severely disabled chronic pain patients with excessive pain behaviors. Following a standardized operant treatment, initial high correlations among these measures were reduced, suggesting that some elements of the treatment induced desynchrony. 
In this study, desynchrony among the components derived from the principal component analysis described in chapter 9 is examined.

\subsection{Method.}

Difference scores for the 7 components are computed by subtracting the mean pre-treatment score from the mean post-treatment score. Kendall correlation coefficients Tau are calculated among these difference scores for the $O P$ and $O C$ treatment groups separately.

\subsection{Results.}

Interestingly enough, 2 different patterns of significant correlations emerge when the correlations for the OC group are compared with those of the OP group. For the OP group, desynchrony occurs between most of the components (Table 12.I). Synchrony occurs only among pain behaviors and health behaviors which are both concepts related to the overt-motoric response system of pain. The greater the reduction of pain behaviors, the greater the increase in health behaviors.

For the OC group synchrony not only occurs between pain behaviors and health behaviors (except health behavior B), but also between pain experience and health behaviors (Table 12.II). The greater increase in health behaviors, the greater decrease in self-reported pain intensity level. Figures 12.1 and 12.2 graphically represent the association between "pain experience" and 'health behavior $\mathrm{A}$ ' for both treatment groups separately.

Table 12.I. Kendall correlation coefficient Tau among component difference scores for the OP group.

\begin{tabular}{lcccrrr}
\hline Component & 2 & 3 & 4 & 5 & 6 & \multicolumn{1}{c}{7} \\
\hline 1. Health Behavior A & $-.54^{* *}$ & $.36^{*}$ & .08 & -.19 & .16 & -.03 \\
2. Pain Behavior & & $-.27^{*}$ & .10 & .23 & -.03 & .02 \\
3. Health Behavior B & & -.17 & -.02 & -.06 & .26 \\
4. Pain Experience & & & .15 & .19 & -.25 \\
5. Negative Affect & & & & .02 & -.01 \\
6. Distorted Cognitions & & & & -.24 \\
7. Reliance on Health Care & & & & & \\
\hline
\end{tabular}

${ }^{*} \mathrm{p}<.05,{ }^{* *} \mathrm{p}<.01,{ }^{* * *} \mathrm{p}<.001$ (one-tailed) 
Table 12.II. Kendall correlation coefficient Tau among component difference scores for the OC group.

\begin{tabular}{lcccccc}
\hline Component & 2 & 3 & 4 & 5 & 6 & 7 \\
\hline 1. Health Behavior A & $-.37^{* *}$ & .04 & $-.44^{* *}$ & -.15 & -.04 & -.05 \\
2. Pain Behavior & & -.18 & -.03 & .21 & .10 & .18 \\
3. Health Behavior B & & & -.10 & -.23 & .10 & -.05 \\
4. Pain Experience & & & .18 & .11 & -.16 \\
5. Negative Affect & & & & .25 & -.06 \\
6. Distorted Cognitions & & & & & -.01 \\
7. Reliance on Health Care & & & & & \\
\hline
\end{tabular}

${ }^{*} \mathrm{p}<.05, * * \mathrm{p}<.01, * * * \mathrm{p}<.001$ (one-tailed)

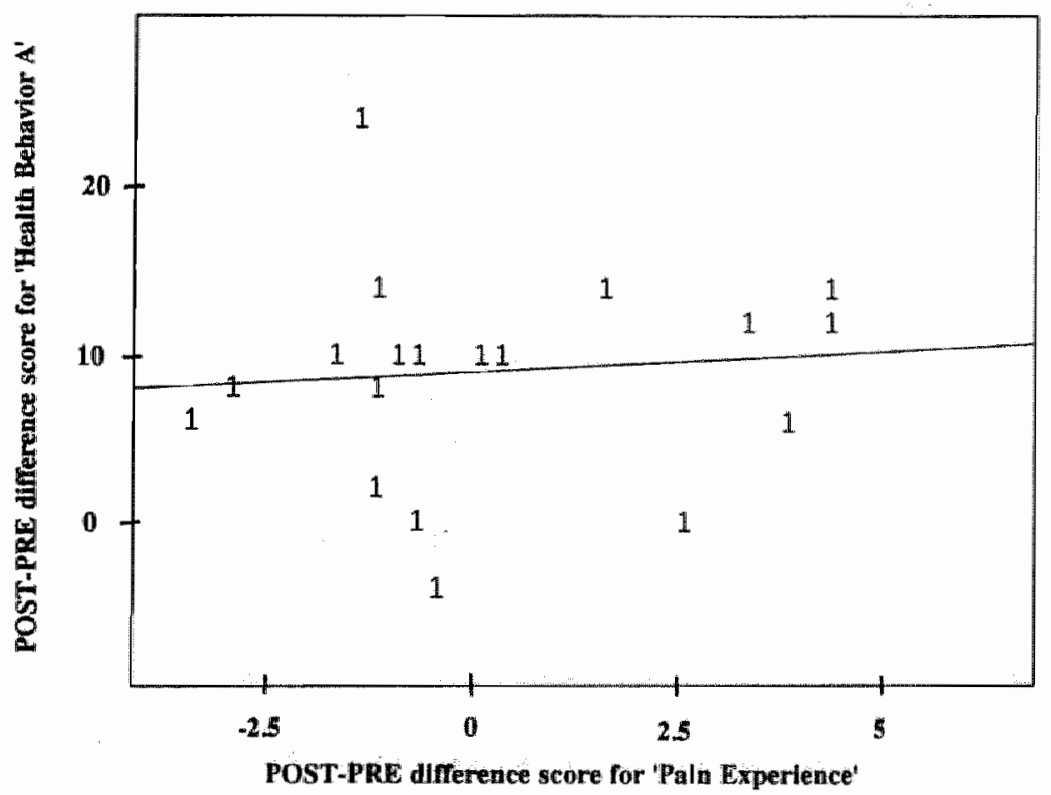

Figure 12.1 Graphical representation of desynchrony between health behavior and pain experience for the $O P$-group.

\subsection{Discussion}

The results suggest that synchrony between the overt-motoric response system and the verbal-cognitive response system of pain only occurs when 


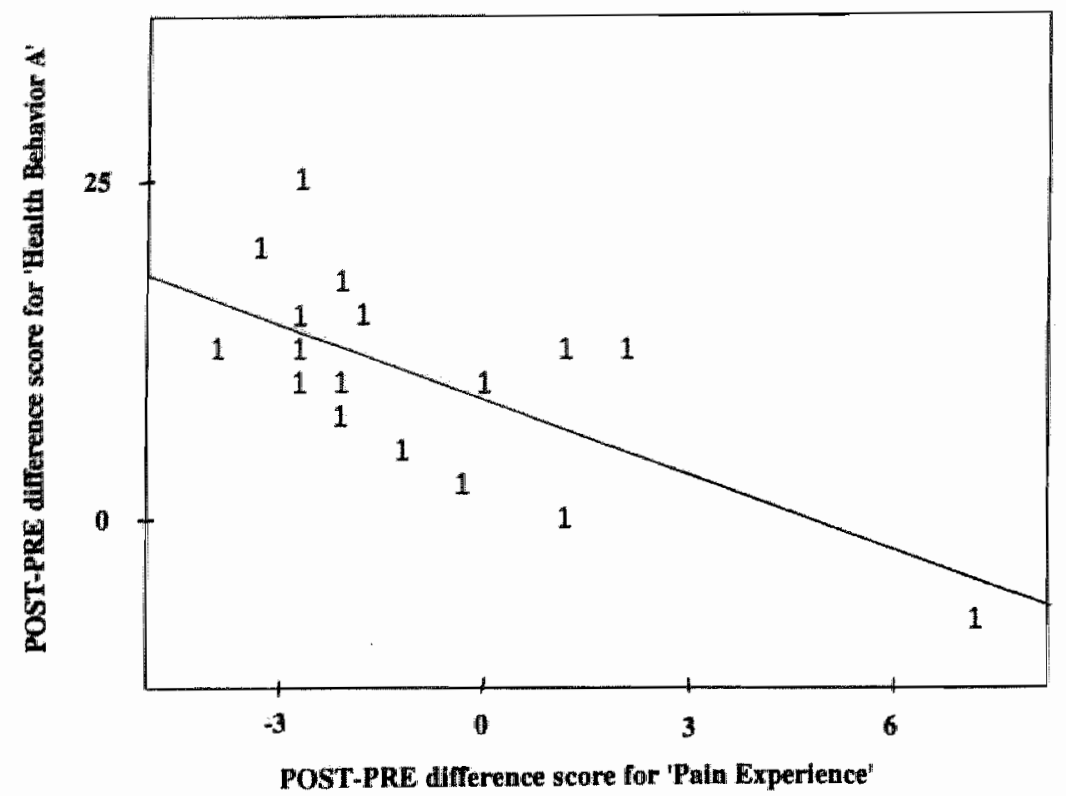

Figure 12.2 Graphical representation of synchrony between health behavior and pain experience for the $O C$-group.

a cognitive treatment, of which the point of application are pain cognitions, is added to an operant treatment. Of interest is, however, the absence of a significant correlation between the change in health behaviors and the change in distorted cognitions. Apparently, the cognitive treatment has more impact on pain experience. Given the non-significant change in pain experience across treatment, the conclusion can be drawn that reported pain experience decreases only for those patients who show substantial increases in health behavior, irrespective of the change in pain behaviors. The operant treatment aimed at increasing health behaviors may be the mediating factor for successful cognitive treatment. Of course, no causal relations may be inferred from correlational studies. Theoretically, it seems important to establish the direction of causality between the different components. Replication of this study with larger pain patient populations is warranted. In future research, clinical scientists will need to evaluate the effectiveness of the component parts of behavioral rehabilitation treatment programs in order to establish which (combination of) components are effective. 


\section{CHAPTER 13}

\section{Methodological justification}

\subsection{Introduction}

The research question of this part was to assess the effectiveness of two behavioral-rehabilitation treatments for patients with CLBP, and to compare the effectiveness of both treatments. Drawing valid inferences about the effects of the intervention requires attention to a variety of factors that potentially obscure the findings. The experiment was set up in such a way to help reduce the plausibility that alternative influences could explain the results. In this chapter, both internal validity and external validity of the experiment will be discussed, and possible threats to these forms of validity pointed out. Internal validity refers to the extent to which the experiment demonstrates unambiguously that the intervention accounts for the change assessed. External validity addresses the extent to which the results of the intervention can be generalized or extended beyond the conditions of the experiment. Possible threats will be examined on the basis of suggestions provided by Cook \& Campbell (1979) and Kazdin (1982).

\subsection{Internal validity}

Given the fact that the patients with CPS in general, and the CLBP patients that participated in our study are therapy-resistent, history and maturation are unlikely to have taken place. No event (other than the intervention) occurring at the time of the experiment could have influenced the results. Theoretically, however, the treatment effects might also be due to the admission at the rehabilitation center per se, and not necessarily to the operant and cognitive treatment ingredients. For a number of practical and ethical reasons, a placebo control group could not be realized. An alternative design, in which a control group receives the traditional rehabilitation treatment without operant conditioning and cognitive treatment cannot be carried out without problems. If the treatments are to be given in the same reha- 
bilitation center, diffusion of treatment is likely to occur: a therapist cannot be expected to reinforce well behavior in one condition and to cease the use of positive reinforcements in another condition.

As is the case with every repeated measures design, changes may be attributed to the effects of repeated assessment. On the other hand, the notreatment WLC group also received 6 measurements. In this way, differential regression between groups is ruled out and the effects of the intervention are separated from the effects of regression. Therefore, it can be concluded that the effects of testing and statistical regression did not play a substantial role.

The potential influence of instrumentation is more relevant in our study. Three kinds of measures were chosen, self-report measures (all questionnaires), observations made by trained observers (PBS, CHIP), and counts made by therapists (MOSOS). For all the process-assessment instruments, reliability and validity have been examined. Although the observers (in casu research assistants) were not associated with the treatment center, they were aware of the assessment phase in which the observations took place, and their expectations might have influenced their ratings. On the other hand, observer training (supported with video samples) was designed to ensure that the observers adhered to the definitions of the behaviors and rated the occurrence of the behaviors at a consistent level of accuracy. It is also possible that the observer has gradually changed his/her criteria for scoring patient performance. A protection against this 'observer drift' was the inclusion of 4 different observers successively, each receiving the observer training, and exchanging their criteria for scoring the observational instruments. Because of the experience that patients may behave differently when they know that they are being observed ("reactivity'), the observations occurred as unobtrusively as possible. For the MOSOS, during which counts are made by therapists, reactivity might have had an effect, especially at the post-treatment and follow-up assessment phases. It is a common procedure to award patients an additional series of booster sessions (at least 6 months after discharge), but only if they were able to stay at their posttreatment level. Some patients might have been on their best behavior to be selected for additional treatment. It is possible that the follow-up data did not correspond with the patients' normal daily behaviors.

Assignment of the subjects to the respective groups did not occur with a randomization procedure. Patients were subsequently selected and accepted for participation in the experiment. Patients who were admitted in the first 18 months of the study were given the OP treatment, and patients who were selected in the second period of 18 months received the OC treatment. Subjects of WLC group follow a different referal channel, and received the 
treatment successfully in the second 18 months of the study. Although the groups may differ as a function of initial selection criteria rather than the treatment conditions, the selection criteria have been held constant during the duration of the experiment (selection biases). Paragraph 9.2.3 and 9.2.4. also showed that no initial differences among the three groups were found on the relevant biographical and personality variables.

Compared to numbers mentioned in the literature, the attrition rate in our study is rather small. Data from the patients who dropped out before the follow-ups were excluded from the analyses. At 6-month follow up, only $11 \%$ did not show up. As some of these patients were good responders, it is unlikely that the relatively good follow-up results are due to the loss of scores that would have been particularly low or high.

Diffusion of treatment occurs when groups receive treatment ingredients that they are not supposed to recieve. In our case, the WLC group was not admitted to the rehabilitation center or any other health care facility where the treatment condition could have been provided. Because a constructive approach was chosen, the $O C$ group did receive the operant treatment ingredient. On the reverse, patients of the OP group could not have received the cognitive treatment ingredient, nor could they have learned it from other patients, as it was not provided in the rehabilitation center at the time the OP group was admitted.

\subsection{External validity}

Generality across subjects refers to the extent to which the results can be extended to subjects whose characteristics may differ from those included in the investigation. Our study deals with relatively small patient samples, which is due to the limited treatment capacity and budget restriction of the rehabilitation center. Nevertheless, a description of biographical variables has been reported in detail, making comparisons with other research populations possible. Typical features of our subject sample is their high mean duration of pain complaints since the onset of back pain (13 years), and the failure to have any further indication for medical treatment. Practically all patients have recelved various monodisciplinary treatments with short-lasting success, or without any success at all. This should be kept in mind when interpreting the results, as they might have been more favorable with subjects who have a shorter medical history. As the subjects' distribution on age, sex and civil status corresponds fairly well with the University Hospital Pain Clinic CLBP patients, generalizability to the Maastricht Hospital Pain Clinic, CLBP population is possible. Finally, the generalizability 
to the clinical situation is high as well, as all patients were referred by the neurologist of the University Hospital Pain Clinic for the experimental groups, and by a rheumatologist of one of the regional hospitals for the WLC group. No single patient was recruited by other means such as the local media, which is often the case in laboratory research.

Generality across settings, responses, and time refers to the extent to which the results extend to other sittuations, other behaviors not included in the program, and beyond the times during the day the intervention is in effect. From the description of the operant treatment program (Supplement 1), it is evident that shaping procedures are applied to functional activities that have a meaning in daily life. The occupational therapy was aimed at applying the newly learned skills into activities of daily life. Nevertheless, generallizability towards other settings would have been greater if the treatment was provided in an outpatient setting. Because of the degree of disability of most of the patients (e.g. many could not walk without supportive equipment), however, it is questionable whether an outpatient operant treatment would have been possible.

Generality across treatment agent refers to the extent to which the intervention effects can be extended to other persons who can administer the intervention. This is a possible threat to the external validity of our experiment that warrants comment. First, the patients were admitted at the department of Rheumatology of the Lucas Foundation of Rehabilitation at Hoensbroek, at which all therapists have considerable experience and expertise in working with people with chronic somatic complaints. Second, both experimental treatments were preceded by extensive inservice training, during which the rehabilitation staff members of all the participating disciplines received training in contingency management techniques (including observation of pain and well behaviors, extinction, shaping, and successive approximation). Finally, during treatment, regular meetings with the clinical psychologist were organized, during which difficulties occurring in the treatments were discussed. The cognitive treatment of the OC condition was given by an experienced clinical psychologist, who was supervised by a member of the Dutch and a member of the Flemish society for Hypnotherapy. It is unlikely that the treatment would have been possible without these extra skills. Therefore, the effects may be restricted to therapists with special skills, training, and expertise.

Reactivity of the experimental arrangement refers to the possibility that subjects are aware that they are participating in an investigation and that this knowledge may bear on the generality of the results. As is the case with all human subjects experiments, an informed consent was obtained from all subjects, including the subjects from the WLC group who granted 
their consent to a different investigation (to examine the natural course of CLBP over a period of 13 weeks). As such, the effect of reactivity cannot be excluded. On the other hand, the effects of reactivity might have been separated from the effects of treatment as all the subjects received the informed consent.

Pretest sensitization is a special case of reactivity. When subjects are assessed before the intervention, the possibility exists that they will be more responsive to the intervention because of this initial assessment. Indeed, during the pre-treatment measurements, which took place at the rehabilitation center, the patients already received the opportunity to get acquainted with the treatment setting and some of its therapists.

Multiple treatment interference arises as a threat when the same subjects receive two or more treatments. The possibility then exist that the particular sequence or order in which the interventions were given may have contributed to the results. In our study, the combined operant-cognitive treatment was considered a different treatment than the operant treatment alone. Therefore, multiple treatment inference is not at issue here.

\subsection{Statistical analyses}

\subsubsection{Variable reduction}

Because of the high number of variables compared to the relatively small number of subjects, it was decided to use composite variable scores. The aim is to select for subsequent analyses a set of measures that is least mutually intercorrelated (Shaffer, 1979). One possibility would be to ask experts in the field to form a priori composite variables, composed of variables that are known to measure different aspects of the same constructs. Sum scores can then be calculated and be used as the new variables under study.

Another alternative is the use of a statistical method to form composite variables. One might examine the correlation matrix based on the data of the 50 patients on one of the measurement points and produce sumscores of variables that intercorrelate highly. With a sufficient number of cases a principal component factor analysis can be used, and the factor scores can serve as the new variables. One problem, however, is that the factor structure found on measurement point 1 may not necessarily mimic the factor structure on the other measurement points. As a result of treatment, the interrelationship among variables is subject to change, affecting the internal consistency of the composite measure on later measurement points.

The second alternative, which has been the method of choice in chapter 
9 , considers any of the repeated measurements as different cases and applies a principal component analysis on the total data. This method takes into account the total variance in the original data, including the variance due to the change of the individ ual variables over time. In this way the minimum number of underlying components which would account for most of that variance can be extracted, and exact measures of the components, rather than estimates, can be obtained. This method has been described by Chapman et al. (1979). A similar method has been used in single subject studies examining intra-individual mood changes: Lebo and Nesselroade (1978) and Zevon and Tellegen (1982) have used the P-technique (Cattell, 1963) involving factoring intraindividual variation and covariation by focusing upon data obtained from measuring one individual with a battery of instruments at many occasions.

\subsubsection{Power}

Because sample size has an effect on the analysis of the data, and because our samples are relatively small, the amount of power available in this study needs consideration.

Power is defined as the probability that a particular statistical test will be able to correctly reject the Null Hy pothesis. This probability is a function of the statistical method employed, the probability threshold at which a result is considered significantly different from randomness (alpha level), the relative strenght or subtlety that an impact will be felt (effect size), and the sample size. In other words, the smaller the sample size, the smaller the probability to detect subtle differences. With small sample sizes, only relatively large effects can be detected.

Because most of the outcome measures used in this study are nearly developed, power calculation can only be tentative. Nevertheless, based on the data of the first measurement, a power calculation can be carried out using the equation described by Bouter \& van Dongen (1988):

$$
N=\frac{2 \sigma^{2}\left(Z_{\alpha / 2}+Z_{Q}\right)}{\delta^{2}}
$$

In this equation, $\mathrm{N}$ is the required number of subjects in each of the groups included in the study, $\delta$ is maximum difference to be detected, and $\sigma$ is the standard deviation which is assumed to be equal in both groups. $Z_{\alpha / 2}$ and $Z_{B}$ are the $Z$-values belonging to the critical levels chosen for $\alpha$ (the probability of making a type I error) and $B$ (the probability of making a type II error). The equation has been proposed to calculate the number of subjects 
needed to be able to detect a certain difference before an experiment is conducted. However, the same equation can be used to calculate the probability of making a type II error of a particular experiment once it has been executed.

Three different group sizes have been included in our study. Most conservatively, this calculation is based on the smallest group size ( $N=13)$. Since no data are available for the composite variables that have been used in our outcome study, data of separate outcome measures are used.

Table 13.I. presents for each measure the maximum change to be detected, the standard deviation, the power based on $\mathrm{N}=13$, and a calculation of $\mathrm{N}$ if a power of 0.90 is to be achieved. It reveals that the power of our study ranges from 0.31 to 0.57 with a mean of 0.39 . This means that the power of our study to detect real differences between the groups is rather low. It is quite possible that some differences between the OP and the OC group are too subtle to be detected with our statistical analysis. To increase the power to 0.90 group sizes should be increased to an average of about 52.

Table 13.I. Power calculation based on data of measurement 1.

\begin{tabular}{lcccc}
\hline Outcome measure & $\begin{array}{l}\text { Maximum change } \\
\text { to be detected }\end{array}$ & SD & $\begin{array}{l}\text { power (1-B) } \\
\text { for } \mathrm{N}=13\end{array}$ & $\begin{array}{l}\text { N for } \\
\text { power=0.90 }\end{array}$ \\
\hline PCL-PI & 5 & 8.72 & 0.31 & 64 \\
PCL-CA & 5 & 7.19 & 0.43 & 44 \\
McGill PRI & 5 & 6.43 & 0.51 & 35 \\
PBS & 1 & 1.77 & 0.30 & 66 \\
& & & & \\
CHIP-DM & 3 & 5.61 & 0.28 & 74 \\
CHIP-VC & 2.5 & 3.56 & 0.43 & 43 \\
CHIP-NC & 2 & 3.38 & 0.33 & 61 \\
& & & & \\
MOSOS WD & 100 & 166.28 & 0.44 & 58 \\
MOSOS-A & 50 & 70.51 & 0.57 & 42 \\
MOSOS-LR & 50 & 59.64 & 0.39 & 30 \\
MOSOS-SWIM & 8.5 & 12.99 & 0.39 & 63 \\
MOSOS-SWIMD & 75 & 114.36 & 0.39 & 49 \\
\hline
\end{tabular}




\subsection{Conclusion}

In the effect study, relatively small numbers of well-described clinically representative CLBP patients were involved. The number of patients who dropped out during treatment or at follow-up was not higher than the numbers mentioned in the literature. Reliable and valid measuring instruments were used that were linked to the basic theoretical and clinical model. The evaluation strategy applied made it possible to answer the questions on efficacy. The treatment procedures investigated were also linked to the underlying theoretical and clinical model and they were representative of procedures described in the international literature. However, for the Dutch health care system, they have been not been carried out in a systematic way. The treatment procedures were applied by experienced therapists with sufficient skills, knowledge and expertise.

The internal validity can be considered acceptable, one possible threat being the observations used. In the future, observations should be made by blind observers who rate video-recorded pain behaviors. In clinical research, questions of external validity may be equally important as internal validity, if not more important. In many instances, clinical research does not permit the luxury of waiting for subsequent studies to show whether the results can be extended to other conditions. In our case, external validity is sufficient if in future applications of the treatments at least two conditions are met:

1. Treatment is carried out by paramedical therapists sufficiently trained in contingency management procedures.

2. The treatment is preceded by one or more measurements.

Both conditions can be considered characteristics of treatment centers with sufficient qualitative standards. Regular in-service training guarantees that the treatment ingredients are made available at all times and by all team members. Pre-treatment assessment is always good practice, also in clinical practice (Kazdin,1982; Barlow et al., 1984).

It is quite possible that more differences between the OP and the OC treatment exist, but that they have not been detected in our study because of the relatively small sample sizes, and hence the limited power of the tests used. In future studies, a power calculation should precede the experiment, based on the data of the newly developed measures now available. It is likely that larger sample sizes will be needed to be able to detect the differences under study. 


\section{PART IV:}

\section{GENERAL DISCUSSION AND CONCLUSIONS}

In this dissertation, an attempt is made to rearrange certain existing concepts of behavioral science, in casu the learning theory, and those of rehabilitation medicine into a single conceptual framework: Behavioral Rehabilitation. It does not have the pretention to provide full empirical evidence for such a framework, although available literature in the area of chronic pain, that supports the framework, is briefly discussed. The theoretical and clinical model outlined in the first two chapters in this book is not totally new. In 1970, the term 'behavioral paediatrics' was introduced (Friedman, 1970), and in 1973, Birk (1973) published a book with the title 'biofeedback: behavioral medicine'. All these approaches have in common the rejection of the traditional biomedical disease model, and the adherence to the biopsychosocial model applied to people with somatic complaints. For chronic pain, biopsychosocial models have had more success in conceptualizing the multifaceted nature of the problem than the traditional disease model (Turk et al. 1983).

Within this behavioral rehabillitation framework, a distinction was made between the functional analysis and the broad-spectrum analysis (holistic theory). The functional analysis focuses on the chronic pain disability, and its purposes are first, to quantify pain behaviors, pain cognitions and expressive physiology, and secondly, to determine whether and how these are maintained by antecedent and/or consequent variables. Throughout this study, the accent has been laid on the first purpose. The second pur- 
pose, the analysis how specific variables control or affect these response systems in specific situations, has been carried out for each individual patient, but representation in this dissertation would be too extensive. The three-systems model of emotions, originated in the research on fear and anxiety, is chosen as the guiding model for the functional analysis. Additionally, the broad-spectrum analysis places the Chronic Pain Syndrome (CPS) in the broader context of rehabilitation-hindering and rehabilitation promoting variables, which, in interaction with disability, may determine the level of handicap. Clusters of problem areas that are derived from the individual broad-spectrum analyses are discussed in chapter 11 .

Consonant with experiences with other behavioral constructs such as anxiety and depression, the position is taken that they are best measured in terms of several of the characterizing dimensions, only one of which is subjective report. Again, the three-systems model of chronic pain was used as the guiding model for the choice and development of assessment instruments.

For the overt-motoric dimension, a new observation scale was developed. (Checklist for Interpersonal Pain behavior, CHIP) that also included a scale for depressive behavior and nervousness. The CHIP was based on an empirical examination of the pain behavior construct. Compared to existing observation scales, such as the Pain Behavior Scale (PBS), the CHIP provides a more comprehensive measure for pain behavior. A recent meta-analysis of non-medical treatments for chronic pain indicated that these treatments reliably affect mood and subjective symptom ratings (Malone \& Strube, 1988). The CHIP offers two measures related to mood (nervousness and depression). The validity of these measures is confirmed in chapter 9. A principal component analysis revealed that, with the VAS, they account for $5.7 \%$ of the variance in a separate component (which was labeled 'negative affect'). Moreover, for this component, the severely distressed subjects score higher than the moderately distressed subjects (Figure 11.2). In accordance with the findings of Malone \& Strube (1988), the component "negative affect' is sensitive to treatment as well. In the Operant treatment $(O P)$ and even more so in the Operant-Cognitive (OC) treatment, a significant decrement on this component is noted.

The clear covariance of the VAS with these observational measures, and not with the MPQ, bears some interesting questions regarding the validity of the VAS. Whatever the patient tries to convey with the VAS appears to be more associated with mood than with the pain experience as measured with the MPQ. Huskisson (1983) suggested that the VAS may not be a good reflection of the condition to be measured. Our findings corroborate this statement. 
A problem with direct observation of pain patients is the cost involved. Obtaining a high quality sample of behavior requires more time than most clinicians have available. Although the CHIP is more comprehensive, the PBS is a valid and reliable measure for pain behavior that can be used in settings were only a short observation time is a vailable. The costs of training observers may be prohibitive. In the future, however, the inclusion of spouses as observers should be examined. Turner \& Clancy (1988) asked spouses to rate patient pain behaviors using a 20 -item pain behavior checklist. Unfortunately, they did not report on the reliability and validity of spouses' ratings of pain behaviors, nor whether an observational skills training was made available for the spouses.

The assessment of pain cognitions is a relatively recent development. (The terms 'pain cognition' or 'cognitive assessment' do not even appear in the subject index of the recent and prestigeous volume 'Issues in Pain Measurement' (Chapman \& Loeser, 1989)). The Pain Cognition List (PCL) was developed to provide information about attributions and expectations in regard to pain. Reulings (1990) carried out an exploratory study comparing the PCL with other recently published pain cognition questionnaires, including the Pain Evaluation Questionnaire (Philips, 1989), the Pain Cognition Questionnaire (Boston et al. 1990), and the Survey of Pain Attitudes (Jensen et al., 1987). She concluded that despite substantial overlap among the questionnaires, they all are tapping different aspects of pain cognitions. The terms 'coping', 'attitude' and 'cognition' are used for the same selfstatements. A concensus in regard to what are considered pain cognitions is still missing. The potential strenght of the PCL is the explicit reference to theoretical model (attribution theory and efficacy expectancy models). The validity of the factor 'outcome efficacy' and the sensitivity of the PCL to changes due to treatment need further investigation.

As a measure of residual health behavior, the MOSOS was developed in the format of a behavioral avoidance test. Sometimes the term 'exercise tolerance test' is used as well (Fordyce, 1976; Karoly \& Jensen, 1987). In the future, this kind of measure should be expanded towards situation and task analysis (Keefe, 1988). If so, rehabilitation goals are more likely to be broken down into specific, concrete and manageable steps. Second, it can keep the focus of assessment on behavior that is likely to have a functional value. Finally, it can shift the attention of the patient away from the elimination of pain towards constructing an expanded repertoire of health behaviors.

Since our study dealt with the tangent plane among the concepts of 'disability", "functional analysis', and the 'three-systems model of emotions", where they are applied to people suffering from the chronic (low back) 
pain syndrome, the assessment has been limited to measures related to these concepts. An exception is chapter 11, where data from a broad-spectrum analysis are included. Quantification on the level of impairment and handicap has been left out of consideration, as well as psycho-physiological measures.

For the level of impairment, Rudy et al. (1990) recently developed a Medical Examination and Diagnostic Information Coding System (MEDICS), which is a weighted scoring approach, based on current medical consensus, that can be used to produce such an index of impairment. In future research, such an approach would be a valuable addition to the current measures.

Quantification of the handicap concept is a difficult enterprise, because of its general and broad definition. An illustration of this broadness is the distinction between 'professionally determined handicap' and 'personally experienced handicap' made by Bangma (1989). Handicap always refers to the discrepancy between the person's abilities and the perceived expectations imposed by social rules and norms. Inclusion of measures of this discrepancy was considered beyond the purposes of this dissertation.

For practical reasons, a measure of the physiological response system could not be included. Early research involving psychophysiological assessment was often simplistic. Flor et al. (1985) and Flor \& Turk (1989) have demonstrated that elevated EMG-levels can be of value, but only when specific and personally relevant stressful stimuli are presented to the patient. As a consequence, assessment of the psychophysiological response system always must be preceded by an identification of these relevent stimuli. This makes the assessment laborious and difficult to carry out in the clinical setting of the rehabilitation center, not to mention the difficulty to have the disposal of appropriate and sensitive electrophysiological measurement devices.

From the three-systems model perspective, behavioral treatments for chronic pain disability can be designed according to the response system which is chosen as the point of application. Operant treatments focus at the overt-motoric response system. Cognitive treatments work with the patients' pain cognitions, and physiological treatments have the arousal level as their point of application.

Following a constructive design, the effectiveness of an operant and an operant-cognitive treatment was examined. Generally, our findings are in accordance with the literature undoubtedly showing that behavioral management programs are effective. Overwhelming evidence speaks to positive outcomes, especially when the long-term nature of the disability and previous treatment failures are considered.

Both the OP and the OC treatments resulted not only in short-term but also long-term improvement on both the motoric as some of the cognitive 
dimensions of the chronic low back pain problem. Increased health behaviors and decreased pain behaviors are observed. Furthermore, negative affect, distorted cognitions, and reliance on health care decreased. Since the changes in pain cognitions occurred in both treatment groups, it might be hypothesized that changes in cognitions followed improved motoric functioning. This is in accordance with findings of Turner \& Clancy (1988) and also consonant with the more general assumption that cognitions can best be modified by treatment techniques that have motoric behavior as their point of application (e.g. Bandura, 1977). Similarly, changes in pain cognitions were not due to decreased pain experience as the level of pain experience remained unchanged. The reported intensity of the pain experience remained unchanged, despite the significant increase in activity levels. Of interest is that even in treatments which do not include restoration of activity levels, patients persist in reporting the same level of pain. An illustration of this finding is given by Spinhoven (1989), who compared the effectiveness of hypnosis and education in a group of CLBP patients.

Besides statistical significance of the changes observed during the treatments, clinical significance of the results has been examined as well. Using criteria based on appropriate reference groups, the subjects of our study achieved clinically relevant post-treatment levels. At the end of treatment, the subjects achieved levels of motoric health behavior that are similar to those of pain-free controls.

Only subtle differences in effectiveness occurred between the operant and the operant-cognitive treatment. The reduction of negative affect was more pronounced in the operant-cognitive treatment, suggesting that the cognitive treatment ingredient might be more responsible for affective changes than the operant ingredient. Furthermore, for the OC group only, changes in pain experience were associated with changes in health behavior. The cognitive treatment may have the unique contribution in having an impact on pain experience, but under the condition that health behaviors increase. An alternative explanation might be that not all OC-subjects benefited from the cognitive treatment, but those who did also improved in health behaviors. Finally, some of the health behaviors increased even more in the $O C$ group. These modest differences corroborate the general finding that, when different pain treatment modalities are compared, few differential effects are found (Cohen et al., 1989). On the other hand, the small sample size and the associated limited power of the tests used may also be responsible for the few differential findings. Subtle differences may have remained undetected. Finally, non-specific factors may be more important than the specific treatment ingredients. To rule out this possibility, future placebo-controlled research will be necessary. 
Although behavioral management programs for CLBP are effective, the important question remains unanswered: what treatments work best under which conditions and for which patients? In order to answer this complex question, carefully designed research with relatively large sample sizes, in which treatment components are clearly defined and differentiated, as well as clear descriptions of patient characteristics, will be necessary. In our study, we only touched the issue by examining the relation between the broad-spectrum analysis and treatment outcome.

The broad-spectrum analysis of our subjects revealed at least two interesting findings which have received little attention in the literature on CLBP so far. First, a high achievement motivation was found among almost all of the subjects. Second, a considerable number of subjects reported phobic complaints, of which fear of body injury was the most predominant.

Achievement motivation is considered a relatively stable personality characteristic and the high level found in our study sample raises the question whether it is one of the mediators in the transition from acute to chronic pain. Subjects who report a high level of achievement motivation are not likely to change their lifestyle easily. On the contrary, they are likely to continue trying to achieve their aspirations and life goals. These people might be of risk to engage in activities that are beyond their capacity and to neglect the acute warning signal, prolonging the healing time unnecessarily, and allowing the development of learning factors that may maintain the pain problem. For these patients, scheduling activities properly might be an important treatment ingredient. The operant treatment program described in appendix $\mathrm{A}$ is highly structured and provides in frequent alternations between periods of rest and activity. The presence of high levels of achievement motivation in our sample is reminiscent of an interesting finding reported by Van Houdenhove et all (1987). These authors found that actionproneness, which is considered a stable parameter of hyperactivity, is higher in chronic pain patients as compared to clinical non-pain control populations. It would be worth examining the relationship between achievement motivation and action-proneness, especially in chronic pain patients.

It is unclear whether the phobic complaints are antecedents or consequences of the chronic pain problem. Although fear was not included as a measure in her studey, Gamsa (1990) suggests that emotional distress in pain patients is more likely to be a consequence than an antecedent of the chronic pain problem. To fully understand the role of emotional distress, prospective studies are needed. Of interest is that phobic complaints, as such, did not hamper treatment. Subjects who reported fears, did as well as the subjects who did not report fear. However, when fear is accompanied 
by other complaints as well, such as depression, problems in social interactions, negativism, and feelings of inadequacy, treatment is hampered. This finding sheds a different light at the working process of the treatments provided. It is very likely that for the phobic patients, the operant treatment has worked as a kind of exposure procedure, while for the moderately distressed patients the operant conditioning element as such might have been the most important. When emotional distress exceeds a certain level, the exposure effect might be counterbalanced by a hindering effect of these complaints, causing relapse. These emotionally distressed patients probably have difficulties in handling the increased confrontation with daily responsibilities as a result of a reduced disability level. For this group of patients, an interdisciplinary pain management program should be followed by a specific and individually tailored treatment that focuses on the additional complaints other than pain. Future research should examine the effectiveness of such a stepwise approach. This kind of valuable research, however, will only be possible when the health care providers create appropriate treatment possibilities for patients with CPS. So far, treatment of psycho-social complaints in people with CPS by the existing mental health centers has not been successful (Geurts et al., 1989).

As budgetary stringency is increasingly felt in the health sector, health care providers are taking into account the economic consequences along with the consequences of therapeutic choices. In a preliminary study (Oosterbos et al., 1990), differences in medical consumption, in terms of medication intake and visits to health care providers, before and after treatment were examined. Subjects were a subgroup of those described in chapter 9 . By means of a structured interview, the patients were asked to retrospectively recall the frequency of their visits to different health care providers 6 months before, and 6 months after treatment. Despite the retrospective nature of this study, the data suggest that both the OP and the $O C$ treatment result in a significant decrease in visits to medical specialists. Interestingly, for the $\mathrm{OC}$ subjects, visits to the general practitioner and physiotherapists have diminished as well. From this point of view, more significant differential effects are found between the OP and the OC group. Because these findings are very promising, a replication with more valid measurement instruments for medical consumption is being carried out.

More research is needed to examine the value of outpatient treatment modalities. Linton \& Götestam (1984) and Linton et al. (1985) have shown that outpatient behavioral management can be about as effective as ordinary (non-behavioral) inpatient rehabilitation treatment. Also here, outpatient programs might be appropriate for some CLBP patients and not for others: In the case where intensive shaping of health behaviors is needed, inpa- 
tient treatment will still be necessary. CLBP patients who also report phobic complaints and who probably benefit from the exposure ingredient of an operant treatment, might be good candidates for an outpatient treatment that focuses on exposure.

Finally, prevention of chronic pain should receive increasing attention, closely connected with research that examines the factors that are responsible for the transition from acute to chronic pain. There is surprisingly little information about the psychological reactions that accompany acute back pain episodes. In acute pain, spontaneous remission as a result of healing is assumed and no behavioral intervention is carried out until pain has persisted for years. However, 'learning' occurs immediately, and does not await the end of the healing process. By the time healing has ended, learning factors, instead of the impairment, may have become responsible for the emerging disability. Early behavioral interventions, such as graded exposure and early return to activity (Fordyce et al., 1986) may be important in limiting the development of chronic pain. Health promotion by professional health care providers at appropriate times might help people how to recognize and to cope with acute pain problems while they are still minor. Prevention programs might include in vivo 'risk analysis', in which coping strategies for specific situations are taught to the individuals at risk (Linton, 1987).

At the same time, resources need to be focused on the identification of those people who are liable to develop chronic pain problems. An important step would be to 'tag' those individuals on the basis of their reaction to acute injury. Early identification of CPS-prone individuals would allow preventive techniques to be focused on them (Philips, 1990).

The increased interest in the application of behavioral science in rehabilitation medicine opens up new perspectives and applications in the area of CLBP, and possibly in other disabilities as well. However, besides their necessary link with a scientific basis these applications must not be carried out without adequate scholarship and sufficient evaluation. Bridging basic research on pain mechanisms and applied research concerned with the assessment and treatment will prevent failure and desillusionment, and enhance care for chronic pain patients. 


\section{APPENDIX A:}

\section{AN OPERANT TREATMENT PROGRAM FOR CLBP PATIENTS}

In this appendix, an operant treatment program aimed at modifying the motoric response system of the pain problem is briefly described. It is a variant of the programs described by Fordyce (1976) and Roberts (1986) that fits into the Dutch health care system, and into a Rehabiliation Center such as the Lucas Foundation for Rehabilitation in particular.

\section{General Features of the operant program}

This 8 weeks inpatient operant program is designed to be carried out by the interdisciplinary staff of the Department of Reumatology of the Lucas Foundation for Rehabilitation at Hoensbroek, The Netherlands. The general goal of the treatment is to increase health behaviors and activity levels, and to decrease pain behaviors and excess disability. The treatment is not aimed at reducing self-reported pain intensity levels. During the first two weeks, only baseline activity levels and pain behaviors are recorded. During this period, a pain-rest contingency schedule is followed: the patient may stop at any time whatever activity he is engaging in. Treatment only starts in the third week with the first patient conference during which treatment goals are discussed with the patient. From then on, an activity-rest contingency schedule is followed: The patient is supposed to meet certain quota of activities, and to rest after termination of these quota. Patients are ad mitted to the program on the condition that their partner has agreed to participate in the spouse course. The treatment is carried out by the whole interdisciplinary rehabilitation staff, including nurses, occupational therapists, physiotherapists, recreational therapists, social worker, psychologist, and physiatrist. 


\section{Establishing baseline levels}

During the first two weeks, no treatment is started yet. The purpose of this period is threefold:

a. The patient has the opportunity to get acquainted with the inpatient setting.

b. During these two weeks therapists are also allowed to perform necessary additional examinations (physical examinations, blood analyses, bicycle ergometer tests, etc..)

c. In order to establish baseline levels, the pain-rest contingency principle is hold throughout this period. During the daily physiotherapy and occupational therapy sessions, the patient is asked to perform several activities with the standard instruction to stop "when pain or physical discomfort prevents you from continueing".

Although it is difficult to accomplish, at this time the therapists are trained not to provide positive reinforcement. The activity levels are recorded graphically. At the same time nurses carefully observe pain behaviors and fill in the CHIP and the PBS. These data are brought to the first staff meeting during which for each patient individual treatment goals are defined.

\section{Defining treatment goals}

At the end of the 2-week baseline period, the data gathered are brought together in the staff meeting during which starting quota, increments, and treatment goals are chosen. The choice of starting quota is usually made as follows. For each activity, the mean baseline level is calculated. From this number, one standard deviation is distracted. The resulting level is chosen as the starting quotum, which is always below the initial exercise tolerance level of the patient. Increments are chosen according to the physical condition of the patient (as judged by the physiatrist and the physiotherapist) and the treatment goals aimed at by the patient and the staff. Treatment goals are chosen to be realistic and functionally applicable in the patients daily life. 


\section{Treatment contract}

The treatment contract is not a legally binding contract, but an understanding between the patient and the rehabilitation team members. It includes a brief description of the general goals of the operant treatment, the way the staff will interact with the patient during the treatment, and the individual quota system. In this contract a clear statement is given of 'what will happen' if the patient engages in, or fails to show, the target behavior. The contract explicitly states that the program is not intended to decrease pain, and that the team members will be socially nonresponsive when pain is communicated verbally or non-verbally. On the other hand, the patient may expect that the therapists will give ample attention when the specific goals, aimed at in a therapy session, have been achieved. The contract also states the consequences of not meeting the quota several times in a row. Finally, for each target behavior, the starting levels, increments, and goals are outlined, so that the patient exactly knows what is expected from him/ her. The contract is signed by the patient, and all of his/her personal therapists.

\section{Performance records and graphs}

Once the treatment has begun, the patient monitors his/her progress by keeping graphs of his/her prescribed activities. The patient may keep a graph of the number of laps walked, the time ridden on a stationary bicycle, or the time worked in a standing position. The graphs are kept in a notebook provided by the therapists and are carried at all times to all assigned treatment activities. The graphs provide feedback not only to the patient but to the entire team. Feedback from the graphs clearly shows the patient's progress. The visualization of this progress is considered an important positive reinforcer for most patients.

\section{Patient conference}

Once a week, the patient and his/her personal therapists meet and examine the graphs representing the progress made during the previous week. At this time, therapists, viewing and commenting on the graphs provide ample positive reinforcement. If necessary, little modifications of the increments or treatment goals are allowed if a concensus among the therapists and the patient is reached. 


\section{Selection of reinforcers}

Theoretically, many reinforcers can be applied, of which social responsiveness is an effective, controllable, and immediately available one. It can be made available to the patient when health behavior is displayed and not at other times. Various forms are possible: giving a compliment, taking time to make a chat, showing interest in the patient activities, and hobby's etc. Contingent attention is given the greatest importance in the early phases of treatment. As progress is observed, social interactions with the patient become more natural, and positive reinforcement is provided on an intermittent schedule.

\section{Shaping}

In case where significant health behavior deficits are observed, shaping procedures are utilized. Health behaviors are then developed by reinforcing successive approximations toward the desirable response. Responses which are dissimilar to the final goal are not reinfored. Chapter 8 describes a shaping program for a patient who was not able to sit and to stand still for more than a couple of seconds. Shaping programs are developed for each patient individually, and are always the result of a coordinated action among the different specialties of the rehabilitation team.

\section{Extinction}

Extinction refers to withholding reinforcement from a previously reinforced response. Initially, the ignored pain behavior may increase before it gradually decreases. Whenever the patient communicates pain in front of a therapist, the latter may look away, start doing something else, or talk about another subject. When these do not suffice, the therapist may leave the room. This does not imply that pain or indications of illness are to be ignored. The team members do not diminish at all their vigilance toward indications that some form of assistance or intervention is needed. If an acute intervention is needed, however, it will be provided on a time-contingent instead of a pain-contingent basis as much as possible. The team members also take care that withholding social responsiveness does not result in a reduction in the amount of contact with the patient. The operant treatment protocol calls for attaching social responsiveness as much as possible to activity and health behavior. 


\section{Spouse training}

Since the social responsiveness of the spouse is as important (if not more important) than of the team, the patient is admitted on the condition that the spouse, or a significant other, participates in the spouse course. This group training consists of 5 weekly sessions of 1 hour. During this training, the spouses learn to recognize the difference between pain behaviors and health behaviors, to be more aware of their own responses towards these behaviors, and finally to positively reinforce health behaviors and ignore pain behaviors.

\section{Maintenance of treatment gains}

An attempt is made to maximize the maintenance of gains made during treatment by using homework assigments directed towards non-pain habits in the home environment. This is done gradually, during the weekends. During the fifth weekend of treatment, the patient goes home one half day, the following weekend a full day and so on, up to a full weekend just before discharge. Before then, the patient has been able to try out his or her new skills at home for at least one full weekend. 'Successful' weekends are only possible with effective activity planning. When a patient 'feels well', he or she may engage in an activity that is beyond his/her capacity, and then 'pays for it' with increased pain. Therefore, guidelines for scheduling activities are discussed with the occupational therapist and the weekends at home are planned jointly with the therapist and the patient. At the time of discharge, the patient also receives a daily program which can be used at home, as a reminder to comply with the exercises initiated during treatment. 



\section{APPENDIX B:}

\section{A COGNITIVE TREATMENT PROGRAM FOR CHRONIC PAIN}

\section{Introduction}

In chapter 2 it was argued that if a three-systems analysis were to reveal that, for each chronic pain patient, one of the three response systems is the most 'disturbed', the treatment would need to be chosen according to the results of this analysis. This implies that treatments can have one of three points of impact: the motoric, the cognitive, and the physiological dimension of the pain. In this appendix, a cognitive treatment module for CLBP patients is described. The program is aimed at decreasing distorted pain attributions and at increasing self-efficacy expectations. Cognitive treatment programs have the advantage to be conducted in an outpatient group setting.

Although there is no one cognitive therapy, common to all cognitive therapies is the modification of the person's thoughts, feelings, and beliefs. In the literature on the behavioral management of chronic pain, a mêlée of different treatment ingredients are often included in what are called cognitive or cognitive-behavioral therapies. In general, cognitive therapy represents a broad class of interventions aimed at alleviating the patient's feelings of helplessness and hopelessness and assisting the patient in gaining control over the pain experience. More specifically, cognitive strategies refer to techniques, used in cognitive therapy, that influence pain through the medium of one's thoughts or cognitions (Jaremko, 1978; Turk et al., 1983). Because of the diversity in existing cognitive therapies, some distinguishing features will be examined first. Secondly, the consecutive steps of a cognitive treatment module developed at the Institute for Rehabilitation Research, in collaboration with the Lucas Foundation for Rehabilitation will be described. 


\section{Cognitive treatments: distinguishing features}

Inspecting the ingredients that are included in existing cognitive therapies, at least four dimensions can be distinguished along which they can be placed: cognitive versus cognitive-behavioral, stress-directed versus paindirected, content-directed versus rationale-directed, and unimodal versus multimodal.

\section{2.a. Cognitive versus cognitive-behavioral}

Cognitive therapies involve verbal methods that try to directly influence thought processes in order to diminish the pain problem. Examples are Rational Emotive Therapy (RET; Ellis, 1973), attention diversion techniques, and hypnotherapy. Cognitive-behavioral therapies are more encompassing: they also involve a variety of behavioral techniques such as relaxation, biofeedback, operant treatment, family therapy, and assertiveness training. According to Turner and Chapman (1982) and Tan (1982), the distinction between the two forms is arbitrary and purely theoretical a distinction that probably cannot be made in practice.

\section{2.b. Stress-directed versus pain-directed}

Stress-directed cognitive treatments aim at teaching the patient techniques which help him/her to cope with stressful events that are thought to be the major factor of the pain problem (Pearce, 1983). These therapies leave from the premise that tension causes physiological changes that contribute to the pain experience. Examples are: RET (Ellis, 1973), stress-inoculation training (Meichenbaum \& Cameron, 1983), and assertiveness training. Paindirected cognitive therapies are aimed at directly changing the quality of the pain experience through techniques such as attention diversion, and relabeling the pain experience through techniques such as hypnotherapy (Stoll, 1985).

\section{2.c. Content-directed versus rationale-directed}

Definitions of cognitive therapy mentioned in the literature offer no direct information of the content of a cognitive therapy. In most cases, the definitions are characterized by the description of the therapy goal: changing cognitions, cognitive restructuring, or changing the pain-experience. Turk et all. (1983) even propose that the therapeutic technique is subordinate to the rationale provided to the patient. In comparing the cognitive behavioral approach with the operant and the transactional, the authors state: 
"Each of the therapies involves a translation process whereby the patients come to view their problems in different terms... The translation is from the terms of physical medicine, with pain seen as a direct result of tissue damage, to the terms of the particular theoretical approach employed....The cognitive-behavioral model underscores the importance of this translation process in the treatment of pain patients." (p.157)

A problem with rationale-directed cognitive therapies is related to their delimitation. In the view of Turk et al., nearly every treatment that is not based on the traditional disease model can be called cognitive. With this definition it is almost impossible to compare the effects of cognitive versus operant treatment programs.

\section{2.d. Unimodal versus multimodal}

A typical unimodal example is the cognitive therapy described and evaluated by Rybstein-Blinchik (1979). She studied separately the effect of three different cognitive strategies on chronic pain patients: relabeling of the sensation (replace the word 'pain' with 'a certain feeling'), objective analysis of the sensation (replace thoughts that accompany experience of pain with new thoughts about important events), and a personally relevant analysis of the sensation (replacing pain thoughts by new and personally relevant ones that reinterpret the pain experience). She found that within a physical rehabilitation hospital setting, patients with a history of persistent pain showed a significant change in their ratings of the quality and intensity of the pain experience.

Most reviews of clinical studies of the effects of cognitive therapy for chronic pain, however, include studies in which cognitive treatments consist of a mêlée of different methods (Tan, 1982; Turner and Chapman, 1982; Turk et al, 1983; Turner and Romano, 1984, Cohen et al., 1989). A typical example of this is a study by Kathami and Rush (1978). The cognitive therapy consisted of relaxation training, EMG-biofeedback, self-hypnosis, self-monitoring, cognitive modification, family therapy and instructions to the family members to ignore pain behaviors and to reinforce healthy behaviors. The difficulty with multimodal treatments is to determine which therapeutic ingredient is most responsible for the outcome. The scientific rule of parsimony argues for research designs that disassemble the total program to determine the effective components. 


\section{A cognitive treatment program}

In this section, the consecutive steps of a cognitive program which is content-directed, pain-directed and unimodal are described. The program consists of 11 sessions of 90 minutes which can be grouped into 3 phases: reconceptualization phase, skills acquisition fase, and a generalization phase. The central feature of the reconceptualization phase is the establishment of a collaborative, participatory relationship between the therapist and the patient. The goal of this phase is to recast the pain experience in terms that imply self-control and resourcefulness. Instead of the patient's viewing pain in global terms, a more differentiated view of the pain experience is presented (Turk et al., 1983). In the skills acquisition phase, the patient exercises cognitive coping skills. In this program, two types of imagery (according to the taxonomy of Fernandez, 1986) are chosen as the main pain coping strategies: 'Transformative imagery' and 'Incompatible sensory imagery'. The first kind of imagery is the imaginative transformation (or relabeling) of the features (intensity, modality, and locus) of the pain sensations. For example, while focusing on the pain, the patient imagines that a hot object is brought close to his skin transforming the pain sensation into warmth. In contrast, when working with incompatible sensory imagery, sensations that are incompatible with the pain are imagined. For example, the patient may be asked to go back in the past and to imagine a pleasant moment, thereby focusing on the pleasant bodily sensations accompanying that image. After some exercises the patient is expected to directly imagine the pleasant sensations.

In our experience vivid imagery is facilitated when the patient is in deep concentration. Although we have chosen not to use the word hypnosis in the presence of our patients, the imagery is preceded by an induction and a deepening procedure similar to the procedures used in hypnotherapy. Hypnotic pain control techniques also include the transformation of pain sensations and the hypnotic images of pleasant experiences that are incompatible with pain or suffering (Udolf, 1981). In this program we have chosen for a hypnotic skills training approach (Diamond, 1977; Golden \& Friedberg, 1986). This approach holds the premise that hypnotic suggestion is a learnable skill rather than an unmodifiable trait, as most traditional hypnotherapists would maintain. Diamond (1977) has developed a skills training approach to enhance the hypnotic responsiveness of clients. Procedures such as modeling, instruction in how to think and imagine along suggestions, and successive approximations whereby clients are reinforced for incremental improvements in hypnotic responsiveness, are taken from his approach. 
An essential component of this cognitive program is the active involvement of the patient outside of the therapy sessions. Homework assignments assist the therapist and the patient in assessing progress and in modifying treatment strategies when necessary. At the end of each session, patients are supplied with texts to read, or a tape to which they are required to listen to at least once a day. When they finish the tape, they write down their experiences, together with an efficacy rating in a specially designed diary. Each session begins with a short discussion of the experiences with the homework assigrment.

\section{3.a. Reconceptualization phase}

\section{Session 1}

In this first meeting the group of patients get acquainted with each other and practical information about the program is given: the duration and number of sessions, the importance of homework assignments, and the use of tapes. Furthermore, information regarding the patients" expectations concerning the program are elicited and questions about the nature of the program are answered. At this point, patients are introcuced in a simple fashion to Melzack and Wall's $(1965,1970)$ gate control model of pain, as illustrated by Turk et al (1983) who discuss various physical, emotional, and mental factors that can open or close the gate. Patients are encouraged to provide specific personal examples that illustrate each factor. A pictoral representation of this model is used, showing the sensory input, the brain, and a gate that can be opened or closed between the sensory input and the brain. This session is closed with the homework assignment consisting of reading a simple text on the issues covered in this session.

\section{Session 2}

This session is devoted to the myths of pain and the difference between acute and chronic pain. It is based on the presentation of these myths offered by Malec et al. (1977) in a cognitive-behavioral bibliotherapy manual for pain patients. Some of these myths include the following:

1. Pain always means that you are hurt and need to rest and take care of yourself until you get better (FALSE!)

Here we also add the illustrations provided by Fordyce (1985) in regard to the distinction between 'HURT' and 'HARM', and the detrimental effects of prolonged rest possibly leading to the disuse syndrome. 
2. If physicians can't cure your pain or find out exactly what is causing it, then your pain must be in your imagination. (FALSE!)

3. Someday, somewhere, someone will find a cure that will quicklly make your pain go away once and for all. (FALSE!)

4. If you can make your pain less by psychological self-control, then the pain was 'all in your head' to begin with. (FALSE!)

Finally, without using the term, the operant conditioning effects are illustrated by means of simple illustations. As in the previous session, the homework assignment consists of reading a simple text on the issues covered in this session.

\section{Session 3}

After reviewing the homework assignments, this session starts with a brief introduction regarding the effects of attention diversion. This introduction focuses on the rationale of attention-diversion techniques, and provides some simple examples. An example which we have found to be quite convincing is the following:

As I am talking to you, your attention is directed to the words I am saying. While listening to me, you are not aware of the position of your left hand, and you are not aware of the tip of your nose. But now, because I just have drawn your attention to your hand and nose, you become more aware of it. Let us now direct our attention to the sounds in this building. You will be surprised how many different sounds can be heard ( 1 minute sillence). For all of us it is impossible to be aware of everything. We would become mad, if at any moment we would be consciously aware of sounds in the external environment, outside this building, in this room, and the things that happen in our body at the same time. We contineously select the things that happen inside or outside our body. This process is called selective attention. Normally, we will select the things that are most striking, such as a bright light, a sudden hard noise, or sharp pain in the body. However, we can exercise control over it. By practice, everyone of us can learn to choose where to direct our attention to. Therefore we will make use of deep concentration and imagery. Let us first do a small exercise on selective attention.

After a brief discussion, during which the therapist examines the patients' experiences with selective attention, the patients listen to a tape, on which a therapist introduces the attention-diversion coping skill. The content is 
based on the script provided by Turk et al. (1983; pp 283-288). Parts of the text are also based on Spinhoven (1989). The first part is informative, the second part is an exercise in imagery. Patients are guided to imagine a pure white plate with a lemon on it. Then they imagine they cut the lemon in two with a sharp knife, pick up one piece and touch their tongue gently against it. The script is designed in such a way that the patients are invited to focus on the different features (color, smell, taste, sound) of the image. The homework assignment consists of listening to the tape again twice.

\section{3.b. Skills acquisition phase 1: Stimulus-transformative imagery}

\section{Session 4}

This session starts with the hypnotic skills training, following the script described by Golden \& Friedberg (1986). Four skills are demonstrated and exercised: 1 . Hand heaviness, 2. Hand levitation, 3. Chevreul's pendulum and 4. Arm catalepsy. In this session, Autogenic training (Schultz, 1963) is started. Relaxation skills are used as induction procedures for the stimulus transformative imagery.

\section{Session 5}

This session focuses on the modification of the pain intensity. After the induction and a deepening procedure, the patient is asked to draw his attention on the pain, and use his concentration and imagery to intensify it. This often is a good demonstration of the control he/she has over his pain. The patient is then told that any sensation that can be increased, can also be diminished. The image that is used is that of the gate, through which the release of endorfins is regulated. First, patients are asked to imagine that the gate closes, as a result of which the pain intensifies. Subsequently, gradual opening of the gate is suggested, resulting in pain diminishing.

\section{Session 6}

This session provides a further exploration of the skills introduced in the previous one. Imagery can also be used to transform pain sensation into other sensations that are easier for the patient to tolerate, such as tingling, itching, or feelings of warmth or cold.

\section{Session 7}

Session 7 is an elaboration of the previous session. In this session imagery is used to transfer the pain from one region of the body to another where it might be less disabling. Pain may be transferred to a smaller and less sensitive region of the body. 


\section{Session 8}

This is the first session dealing with distraction techniques which involve the use of imagery to focus on pleasant experiences that are incompatible with pain or suffering. By concentrating on these pleasant experiences the patient is able to move the pain towards the background. In planning this type of suggestion the patients are asked in advance about what types of experience they enjoy most, to maximize the pleasant effect sought and to avoid the possibility of suggesting something that a particular patient might find upsetting. Pleasant sensations experienced during the autogenic training can be chosen, as at this time, patients have had enough experience with this induction method. Patients are also encouraged to visualize the experience on an imagined movie screen or TV set.

In this session the distraction technique is preceded by an induction, a deepening procedure and one of the exercises of the previous sessions (transformative imagery). These intermediate steps will successively be omitted in the following sessions.

\section{Session 9}

This session consists of a rehearsal of the previous session, but with the omission of the transformative imagery exercise. After the induction and the deepening procedure, the suggestion is given to think of a pleasant experience of the past and then concentrate on the accompanying pleasant bodily sensations.

\section{Session 10}

In this session, the induction and deepening procedure is directly followed by the concentration on pleasant sensations. As this exercise is much shorter than those of the previous sessions, it can be done twice.

\section{3.d. Generalization phase}

\section{Session 11}

During this last session, the cognitive program is evaluated. In the previous session patients were requested to go over the different exercises and decide which exercise they found most useful. The information is used to design an individually tailored audiotape which the patient can use whenever he likes after his discharge. 


\section{LITERATURE}

Abelson, I.L. \& Curtis G.C. (1989) Cardiall and neuroendocrine responses to exposure therapy in height phobics: Desynchrony within the "physiological response system'. Behaviour Reseatch and therapy, vol 27, 5, 561-567.

Alpert, R. \& Haber, R.N. (1960) Anxiety in academic achievement situations. Journal of Abnormal Psychology, 61, 207-215.

Arntz, A. (1989) Cognitive aspects of the processing of repeatind painfull experiences. Paper presented at the First World Congress on Cognitive Therapy. Oxford.

Arntz, A., van Eck, M., Heijmans, M. (1990) Predictions of dental pain: the fear of any expected evil is worse than the evil itself. Behawiour Research and Therapy, 28, 29-41.

Arrindell, W.A. (1980) Dimensional structure and psycho-pathology correlates of the FSS-III in a phobic population: a factorial definition of agoraphobia. Behaviour Research and Therapy, 18,229-242.

Arrindell, W.A. \& Zwaan, E.J. (1982) Fobische dimensies, de AZU-Vreesvragenlijst (AZUV) en Wolpe's en Lang's Fear Survey Schedule (FSS-III). Nederlands Tijdschrift voor Psychologie, 37, 225-239.

Arrindell, W.A., de Groot, P.M. \& Walburg, J.A. (1984) De Schaal voor Interpersoonlijk Gedrag (SIG), Handleiding. Lisse: Swets \& Zeitlinger.

Arroyo, P. (1966) Electromyography in the evaluation of reflex muscle spasm. journal of Florida Medical Association, 53, 29-31.

Atkinson, J.W. \& Feather, N.T. (1966) A theory of achievement motivation. New York: Wiley.

Bakal, D.A., Demjen, S. \& Koganov, J. (1984) The continuous nature of head achesusceptibility. Social Sciences in Medicine, 12, 1305-1311.

Bandura, A. (1977) Self-efficacy: towards a unifying theory of behavior change. Psychological Review, 84, 191-215.

Bandura, A. (1978) Perceived self-efficacy. In: S. Rachman (Ed.) Advances in Behaviour Research and Therapy, 1, 4.

Bangma, B.D. (1989) Rezalidatie-Gneeskunde. Methodologie en praktische uitwoering. Assen: Van Gorcum.

Barlow, D.H., Mavissakalian, M.R. \& Schofield, L.D. (1980) Patterns of desynchnony in agoraphobia: A preliminary report. Behaviour Research and Therapy, 18, 441. 448.

Barrios, B. \& Hartmann, D.P. (1986) The contributions of traditional assessment: concepts, issures and methodologies. In: R.O. Nelson and S.C. Hayes (Eds.) Conceptual foundations of behawioral assessment. New York: The Guilford Press.

Beck, A.T., Rush, A.J., Shaw, B.F. \& Emery, G. (1979) Cognitive Therapy of Depression. New York: Wiley \& Sons.

Beecher, H.K. (1956) Relationship of significance of wound to the pain experienced. Journal of the American Medical Association, 161, 1609-1613.

Beecher, H.K. (1959) Measurement of Subjectize Responses. New York: Oxford University Press.

Belkin, S. (1985) Back pain. In: G.M. Aronoff (Ed.) Evaluation and Treatment of Chronic Pain. Baltimore-Munich: Urban \& Schwarzenberg. 
Bellack, A.S. \& Hersen, M. (1988) Behavioral assessment, a practical handbook. New York: Pergamon Pressi.

Bernstein, D.A. Nietzel, M.T. (1974) Behavioral avoidance tests: the effects of demand characteristics and repeated measures on two types of subjects. Behavior Therapy, $5,183-192$.

Birk, L. (1973) Biofeedback: Behaviaral Medicine. New York: Grune \& Stratton.

Block, A.R., Kremer, EF. \& Gaylor, M. (1981) Behavioral treatment of chronic pain: The spouse as a discriminative cue for pain behavior. Pain, 9, 243-252.

Bonica, J.). (1985) Introduction. In: G.M. Aronoff (Ed.) Evaluation and treatment of chronic pain. Baltimore-Munich: Urban \& Schwarzenberg.

Bortz, W.M. (1984) The disuse syndrome. Western Journal of Medicine, 141, 691-694.

Boston, K., Pearce, S.A. \& Richardson, P.H. (1990) The Pain Cognitions Questionnaire Joumal of Psychosomatic Research, 34, 1, 103-109.

Bouter, L.M. \& van Dongen, M.C.J.M. (1988) Epidemiologische onderzoek. Opzet en interpretatie. Utrecht: Bohn, Scheltema \& Holkema.

Bradley, L.A. \& Van der Heide, L.H. (1984) Pain-related Correlates of MMPI profile subgroups among back pain patients. Health Psychology, 3, 157-174.

Bremer, J.J.C.B. (1972) De ziekenhuispatient. Een hoofdstuk uit de medische psychologie. Nijmegen: Dekker \& van de Vegt.

British Medical Association Report (1955). Medical use of hypnolism. British Medical Journal, 1, Supplement, 190-193.

Brockway, J.A. \& Steger, J.C (1981) Chronic operant pain. In: J.L. Shelton \& R.L. Levy (Eds.) Behavioral Assignments and Treatment Compliance, A Handbook of Clinical Strategies. Champaign, Illinois: Research Press.

Brown \& Sons, Inc. (1987, August 4) Comprehensive Medical Rehabilitation: An industry taking shape. Research Health Care Group Industry.

Cairms, D. \& Pasino, J.A, (1977) Comparison of verbal reinforcement and feed back in the operant treatment of disability due to chronic low back pain. Behavior Therapy, $8,621-630$.

Cattell, R.B. (1963) The structuring of change by P-technique and incremental-R technique. In C.W. Harris (Ed.) Problems in measuring change. Madison, Wisconsin: University of Wisconsin Press.

Cautela, J.R. \& Kearney, A.J. (1986) The covert conditioning handbook. New York: Springer Publishing Company.

Cautela, J.R. (1977) The use of covert conditioning in modifying pain behavior. Journal of Behavior Therapy and Experimental Psychiatry, vol 8, 45-52.

Chapman, C.R., Casey, K.L., Dubner, R., Foley, K.M., Gracely, R.H. \& Reading, A.E. (1985) Pain measurement: an overview, Pain, 22, 1-13.

Chapman, R.M., McCrary, J.W., Bragdon, H.R. \& Chapman, J.A. (1979) Latent components of event-related potentials functionally related to information processing. In; J.E. Desmedt (Ed.) Cognitive components in cerebral event-related. potentials and selective attention. Progress in Clinical Nenrophysiology, vol 6. Basel: S. Karger.

Chapman, C.R. Solla, A.E. \& Bonica, J.J. (1979) Illness behavior and depression compared in pain center and private practice patients. Pain, 6, 1-7.

Chapman, C.R. \& Wyckoff, M. (1981) The problem of pain: a psychobiological perspective. In S.N. Haynes \& L. Gannon (Eds.) Psychosomatic disorders: a psychophysical approach to etiology and treatment. New York: Praeger. 
Cicala, R.S. (1990) Reply to Yanagida. Letter to the Editor. The Clinical Jowrnal of Pain, 6,161 .

Cohen, M.J., Naliboff, B.D. \& McArthur, D.L. (1989) Implications of medical and biopsychosocial models for understanding and treating chronic pain. Critical Reviews in Physical and Rehabilitation Medicine, wol 1, 3, 135-160.

Collins, G.A., Cohen, M.J., Naliboff, B.D. \& Schandler, S.L. (1982) Comparative analysis of paraspinal ard frontalis $E M G$, heart rate and skin conductance in chronic low back pain patients and normals to various postures and stress. Scandinavian Journal of Rehabilitation Medicine, 14, 39-46.

Cook, T.D. \& Campbell, D.T. (1979) Quasi-experimentation: Design and analysis issues for field settings. Chicago: Rand McNally.

Cosyns, P. \& Vlaeyen, J. (1983) La douleur chronique. In: Fontaine et al. (Eds.) Cliniques de Therapies Comportementales. Liège: Mardaga.

Craig, K.D. (1978) Social modeling influences on pain. In: R.A. Sternbach (Ed.) The Psychology of pain. New York: Raven Press.

Cram $_{*}$ J. \& Steger, J. (1983) EMG scanning in the diagnosis of chronic pain, Biofeedback and Self-regulation, 8,229 -

Cronbach, L.J. (1971) Test validation. In: R.L. Thorndike (Ed.) Educational measurement ( 2 nd edition). Washington DC: American Council on Education.

Crook, J. \& Tunks, E. (1985) Defining the 'Chronic Pain Syndrome'. An epidemiological method. The Clinical Journal of Pain, 1, 159-163.

Crue, B.L. (1985) Foreword. In G.M. Aronoff (Ed.) Evaluation and treatment of chronic pain. Baltimore: Urban \& Schwartzenberg.

Davey, G.G.L. (1987) An integration of human and animal models of Pavlovian conditioning: Associations, cognitions and attributions. In: G. Davey (Ed.) Cognitive Processes and Pavlovian Conditioning in Humans. Chichester: Wiley.

DeAngelis, T. (1989) Playfulness in therapy opens new dimensions. The American Psychological Association Monitor, 20, 27.

Davies, P.M. \& Coxon, A.P. (1983) MDS (X) User Manual. The MDS (X) Series of Multidimensonal Scaling Programming. Inter-University Research Council Series, Report no. 55, University of Edinburgh.

DeVries, H.A. (1966) Quantitative electromyographic investigation of the spasm theory of muscle pain. American Journal of Physical Medicine, 45, 119-134.

Deyo, R.A., Bass, J.E., Walsh, N.E., Schoenfeld, L.S. \& Ramamurthy, S. (1988) Prognostic variability among chronic pain patients: Implications for study design, interpretation, and reporting. Archives of Physical Medicine and Reha* bilitation, 69, 174-178.

Diamond, M.J. (1977) Hypnotizability is modifiable: An alternative approach. International Journal of Clinical and Experimental Hypnosis, 25, 147-166.

Dolce, J.J., Raczynski, J.M. (1985) Neuromuscular activity and electromyography in painful backs: Psychological and biomechanical models in assessment and treatment. Psychological Bulletin, 3, 502-520.

Dolce, J.J., Crocker, M.F. \& Doleys, D.M. (1986) Prediction of outcome among chronic pain patients. Behaviour Research and Therapy, 24, 3, 313-319.

Dolce, J.J., Crocker, M.F., Molettaire, C. \& Doleys, D.M. (1986) Exercise quotas, anticipatory concern and self-efficacy expectancies in chronic pain: a preliminary report. Pain, 24, 365-372.

Dolce, J.J., Doleys, D.M., Raczynski, J.M., Lossie J., Poole, L. \& Smith, M. (1986) The 
role of sielf-efficacy expectancies in the prediction of pain tolerance. Patin, 27, 261272 .

Dolce, II. (1987) Selfefficacy and disability bellefs in behavioral treatment of pain. Behavior Research and Therapy, 4, 289-299.

Doleys D.M. Crocker M. Patton, D. (1982) Response of patients with chronic pain to exercise quotas. Physical Therapy, 8: 1111-1114.

Ells, A. (1973) Humanistic Psychotherapy: The rational-enotive approach. New York: McGraw Hill.

Engel, G.L. (1959) 'Psychogenic pain and the pain-prone patient. American Journal of Medicine, $26,899-918$.

Espie, C.A. \& Brooks, D.N. (1989) An evaluation of taillored psychological treatment of insomnia. Journal of Behavior Therapy and Experimental Psychiatry, vol 20,2, 143-153.

Everitt, B. (1974) Cluster Analysis, London: Heinemann.

Fairbank J.C.T. Davies J.B., Couper J \& O'Brien J.P. (1980) The Oswestry low back pain disability questionnaire. Physiotherapy 66, 271-273.

Fernandez, E. (1986) A classification system of cognitive coping strategies for pain. Pain, 26, 141:152.

Ferster, C.B. (1965) Classification of behavioral pathology. In. L. Krasner \& L.P. Ullmann (Eds.) Research in behavior modification. New York: Holt, Rinehart \& Winston.

Feuerstein, M., Greenwald, M., Gamache, M.P., Papciak, A.S. \& Cook, E.W. (1985) The Pain Behavior Scale: Modification and validation for outpatient use. Joumal of Psychopathology and Behavioral Assessment, 7, 301-315.

Fishbein, P.A. \& Rosomoff, H.L. (1990) Reply to Yanagida. Letter to the Editor. The Clinical Journal of Pain, 6, 164-166.

Flor, H. \& Turk, D.C. (1984) Etiological theories and treatments for chronic back pain. I. Somatic models and interventions. Pain, 19, 105-121.

Flor, H., Turk, D.C., Birbaumer, N. (1985), Assessment of stress-related psychophysiological reactions in chronic back pain patients. Journal of Consulting and Clinical Psychology, 53, 354-364

Flor, H. \& Turk, D.C. (1988) Chronic back pain and rheumatoid arthritis: Predicting pain and disability from cognitive variables. J. Behav. Med. 11, 251-265.

Folkman, S Lazarus, R.S. (1980) An analysis of coping in a middle-aged community sample. Journal of Health and Social Behavior, 21, 219-239.

Fordyce, W.E., Fowler, R.S. \& DeLateur, B.J. (1968) Some implications of learning in problems of chronic pain. Behaviour Research and Therapy, 6: 105-7.

Fordyce, W.E., Fowler, R.S., Lehman, J. F., Delateur, B.J., Sand, P.L. \& Trieschmann, R.B. (1973) Operant conditioning in the treatment of chronic pain. Archives of Physical Medicine and Rehabilitation, 54, 399-408.

Fordyce, W.E. (1976) Behavioral wethods for chronic pain and ilness. St. Lou is: The Mosby Company.

Fordyce, W.E., Shelton, J.L., Dundore, D.E. (1982) The modification of a voidance Learning in Pain behaviors. Journal of Behavioral Medicine, $5,405-414$.

Fordyce, W.E., Lansky, D., Calsym, D.A., Shelton, J.L., Stolov, W.L. \& Rock, D.L. (1984), Pain measurement and pain behavior. Pain, 18, 53-69.

Fordyce, W.E., Brockway, J., Bergman, J. \& Spengler, D. (1986) A control group comparison of behavioral versus traditional management methods in acute low back pain. Journal of Behavioral Medicine, 2, 127-140. 
Fordyce, W.E. (1988) Pain and Suffering: a reappraisal. American Psychologist, 43, 276283.

Frey von M. (1896). Untersuchungen ither die Sinnesfunctionen der menschichen Hawt. Des XXIII. Bandes der Abhandlungen der mathematisch-physischen Classe der Königl. Sächsischen Gesellschaft der Wissenschaften, nr. II. Leipzig: Hirzel.

Friedman, S.B. (1970) The challange in behavioral paediatrics. Journal of Patdiatrics, 77, 172-173.

Gallagher, R.M. (1990) Reply to Yanagida. Letter to the Editor. The Clinical Journal of Pain, 6, 161-162.

Gamsa, A. (1990) Is emotional disturbance a precipitator or a consequence of chronic pain? Pain, 42, 183-195.

Gannon, L.R. \& Haynes, S.N. (1986) Cognitive-physiological discordance as an etiological factor in psychophysiologic disorders. In: S. Rachman (Ed), Advances in Behavior Research and Therapy, voll 8, 223-236.

Gentry, W.D. \& Bernal, G. (1977) Chronic pain. In: R. Williams \& W.D. Gentry (Eds.) Behavioral approaches to medical treatment. Cambridge, Mass.: Ballinger, 173-182.

Gerew, A.B., Romney, D.M. \& Leboeuf, A. (1989) Synchrony and desynchrony in high and low arousal subjects undergoing therapeutic exposure. Journal of Behavior Therapy and Experimental Psychiatry, 20, 1, 41-48.

Geurts, S.M., Gerards, F. \& Schuerman, I.A. (1989) Continuity of psycho-social care for patients with chronic low back pain after discharge from a rehabilitation centre. Journal of Rehabilitation Sciences, 2, 84-88.

Golden, W.L. \& Friedberg, F. (1986) Cognitive-Behavioral Hypnotherapy. In: W. Dryden \& W.L. Golden (Eds.) Cognitive-behavioral approaches to psychotherapy. London: Harper \& Row.Gough, H.G. (1964) Manual for the California Psychalogical Inventory. Palo Alto: Consulting Press.

Granger, C.V. (1984) A conceptual model for functional assessment. In: C.V. Granger \& G.E. Gresham (Eds.) Functional Assessment in Rehabilitation Medicine. Baltimore: Williams \& Wilkins.

Groenman, N.H., Schuerman, J.A., Vlaeyen, J.W.S. \& van Eek, H. (1986) Chronische pijn. In: A.A. Kaptein et al. (Eds) Behavioral medicine „Psychologische behandeling van lichamelijke aandoeningen. Alphen a/d Rijn: Stafleu.

Greenberg, L.S. \& Safran, J.D. (1984) Integrating affect and cognition: A perspective on the process of therapeutic change. Cognitive Therapy and Research, vol 8,6 , 559-578.

Grzesiak, R. (1977) Relaxation techniques in the treatment of chronic pain. Archives of Physical and Medical Rehabilitation, 58, 270-272.

Haanen, H.C.M. (1984) Een epidemiologisch onderzoek naar lage rugpijn (an epidemiological survey on low back pain). Dissertation. Rotterdam: Erasmus University.

Hackett, G. \& Horan, J.]. (1980) Stress-inoculation for pain: What's really going On? Journal of Counseling Psychology, 27, 107-116

Halfens, R.J. (1985) Locus of control. De beheersingsoriëntatie in relatie tot ziekte-em gezondheidsgedrag. Dissertation. Maastricht: University of Limburg.

Hall ${ }_{*}$ C.S. \& Lindzey, G. (1978) Theories of personality. Third edition. New York: Willey. Hand, D.J. \& Taylor, C.C. (1987) Multivariate Analysis of Variance and Repeated Measures. A practical approach for behavioural scientists. London: Chapman \& Hall. 
Hathaway, S.R. McKinley, J.C. (1967) Minnesota Mulitiphasic Personality Inventory (revised edition). New York: Psychological Corporation.

Hayes, S.C. Nelson, R.O. \& Jarrett, R.B. (1987) The treatment utility of assessment, American Psychologist, 11, 963-974.

Heinrich, R.L., Cohen, M.J., Naliboff, B.D., Collins, G.A. \& Bonebakker, A.D. (1985) Compa ring physical and behavior therapy for chronic low back pain on physical abilities, psychological distress, and patients' perceptions. Jourmal of Behavioral Medicine, 8, 61-78.

Hermans, H.J.M. (1976) PMT, Prestatie Motioatie Test, Handleiding. Lisse: Swets \& Zeitlinger.

Hilgard, E.R. \& Hilgard, J.R. (1975) Hypnosis in the Relief of Pain. Los Altos: William Kaufman.

Hodgson, RI. \& Rachman, S. (1977) Obsessional-Compulsive Complaints. Behaviour Research and Therapy, vol 15, 389-395.

Hodgson, R.J. \& Rachman, S. (1984) Desynchrony in measures of fear, Behaviour Research and Therapy, 12, 319-326.

Hugdahl, K. (1981) The Three-systems-model of fear and emotion, A critical examination. Behaviour Research and Therapy, 19, 75-85.

Huskisson, E.C. (1983) Visual analog scales. In: R. Melzack (Ed.), Pain Measurement and Assessment. New York: Raven Press.

International Association for the Study of Pain, Subcommittee on Taxonomy (1986) Classification of chronic pain, descriptions of chronic pain syndromes and definitions of pain terms, Pain, supplement 3, S1-S225.

James, F.R., Large, R.G. \& Beale, J.L. (1989) Self-hypnosis in chronic pain. A multiple baseline study of five highly hypnotisable subjects. The Clinical Jounnal of Pain, 5, 161-168.

Jaremko, M.E. (1978) Cognitive strategies in the control of pain tolerance. Journal of Behavior Therapy and Experimental Psychiatry, 9, 239-244.

Jensen, M.P. \& Karoly, P. (1989) Revision and cross-validation of the Survey of Pain Attitudes (SOPA). Paper presented at the 10th Annual Meeting of the Society of Behavioral Medicine. San Francisco.

Kanfer, F.H. \& Phillips, J.S. (1970) Learning foundations of behavior therapy. New York: Willey \& Sons.

Karoly, P. \& Jensen, M.P. (1987) Multimethod Assessment of Chronic Pain. Oxford: Pergamon Press.

Kathami, M. \&ush, A.I. (1978) A pilot study of the treatment of outpatients with chronic pain. Symptom control, stimulus control and social system intervention. Pain, 5, 163-172.

Kazdin, A.E. (1980) Behavior Modification in Applied Settings. Revised edition. Homewood, Illinois: Dorsey Press.

Kazdin A.E.(1982) Single-Case Research Designs. Methods for Clinical and Applied Set tings. New York: Oxford University Press.

Keefe, F.J. (1982) Behavioral assessment anf treatment of chronic pain. Current status and future directions. Jowrnal of Consulting and Clinical Psychology, vol 50, $6,896-911$.

Keefe, F.J. (1988) Behavioral measurement of pain. In: C.R. Chapman \& J.D. Loeser (Eds.) Issues in Pain Measurement. New York: Raven Press.

Keefe, F.J., Shapiro, B., Brown, C., Williams, R.B. \& Surwit, R.S. (1981) EMG assisted relaxation training in the management of chronic low back pain. American 
Journal of Clinical Biofeedback, $4,93-103$.

Keefe, F.J. \& Brown, C.J. (1982) Behavioral treatment of chronic pain syndromes. In: P.A. Boudewyns \&.J. Keefe (Eds.) Behavtoral medicine in general medical practice. Menlo Park California: Addison-Wesley.

Keefe, F.J. \& Dolan, E. (1986) Pain behavior and pain coping strategies in low back pain and myofacial pain dysfunction syndrome patients, Pain, 24,49-56.

Keefe, F.J., Crisson, J.E. \& Trainor, M.T. (1987) Observational methods for assessing pain: A practical guide. In: J.A. Blumenthal, \& D. McKee (Eds.) Applications in behavioral medicine and health psychology: A clinician's sourcebook (pp. 67-94). Sarasota, Florida: Professional Resource Exchange.

Kendall, P.C. \& Korgeski, G.P. (1979) Assessment and cognitive-behavioral interventions. Cogn. Ther. Res., 3, 1-21.

Kerlinger, F.N. (1986) Foundations of behavioral research (third edition). New York: Holt, Rinehart \& Winston.

Kerns, R.D., Turk, D.C., Holzman, A.D. \& Rudy, T.E. (1986) Comparison of cognitive-behavioral and behavioral approaches to the outpatient treatment of chronic pain. Clinical Journal of Pain, 1, 195-203.

Kincey, J. \& Benjamin, S. (1984) Desynchrony following the treatment of pain behaviour. Behaviour Research and Therapy, 22, 1, 85-86.

Kleinke, C.L. \& Spangler Jr., A.S. (1988) Psychometric analysis of the audiovisual taxanomy for assessing pain behavior in chronic back pain patients. Journal of behavioural Medicine, 11, 83-93.

Kolb, L.C. (1954) The painful phantom: psychology physiology and treatment. Springfield, Illinois: Charles C. Thomas.

Kole-Snijders A.M.J., J.W.S. Vlaeyen, H. van Eek, J.A. Schuerman \& N.H. Groenman (1990) Cognitive behandeling van chronische pijn: Een bron van verwarring. Gedragstherapie, 23, 3-16.

Kraaykamp, H., Emmelkamp, P.M.G. \& van den Hout, M.A. (1990) The Maudsley Obsessional-Compulsive Inventory: Reliability and Validity. Unpublished Manuscript. Groningen: State University.

Krämer ${ }_{\varkappa}$ J. (1981) Intervertrebral disc diseases. Stuttgart: Thieme.

Krech, D., Crutchfield, R.S. \& Livson, N. (1974) Elements of psychology (third edition). New York: Alfred A. Knopf.

Kremer, E.F., A. Block, \& M.S. Gaylor (1981) Behavioral approaches to treatment of chronic pain: The inaccuracy of patient self-report measures. Archives of Physical and Medical Rehabilitation, 62, 188-191.

Kruskal, J.B. \& Wish, M. (1978) Multidimensional Scaling. Beverly Hills, California: Sage.

Kuhn, T.S. (1962) The structure of scientific rewolutions. Chicago: University of Chicago Press.

Lang, P. (1968) Fear reduction and fear behavior: Problems in treating a construct. Research in Psychotherapy, 3, 90-102.

Lang, P.J. (1978) Anxiety: Toward a psychophysiological definition. In: H.S. Akiskal \& W.L. Webb (Eds.) Psychiatric diagnosis: exploration of biological predictors. New York: Spectrum Publications.

Lang, P. \& Lazovik, A.D. (1963) Experimental desensitization of a phobia. Journal of Abnormal and Social Psychology, 66, 519-525.

Langrana, N.A. \& Lee, C.K. (1984) Isokinetic evaluation of trunk muscles. Spine, 9 , $171-175$. 
Lankhorst, G.I., Stad R.I., Vogelaar, T.W., van der Korst, J.K. \& Prevo, A.J.H. (1983) The effect of the Swedish back school in chronic idiopathic low back pain. Scandinavian Journal of Rehabilitation Medicine, 15, 141-145.

Large, R.G. \& Lamb, A.M. (1983) Electromyografic (EMG) feedback in chronic musculloskeletal pain: a controlled trial. Pain, 17, 167-177.

Lazarus, R.S. (1984) On the primacy of cognition. American Psychologist, 39, 124-129.

Lazarus, R.S. Averill J.R. \& Opton E.M. (1970) Towards a cognitive theory of emotion. In M.B. Arnold (Ed.) Feelings and Emotions. The Loyola Symposium. New York: Academic Press.

Lebo, M.A. Nesselroade, J.R. (1978) Intraindividual differences dimensions of mood change during pregnancy identified in five P-technique factor analyses. journal of Research in Personality, 12, 205-224.

Lee, C. (1989) Theoretical weakness lead to practical problems: The example of selfefficacy theory. Joumal of Behavior Therapy and Experimental Psychiatry. vol 20 , 2, 115-123.

Lefcourt, H.M. \& Martin, R.A. (1986) Human and Life Stress, Antidote to Adversity. New York: Springer Verlag.

Lefebvre, MF. (1981) Cognitive distortion and cognitive errors in depressed psychiatric and low back pain patients. Journal of Consulting and Clinical Psychology, 49,517-525.

Lethem, J., P.D. Slade, J.D.G. Troup, \& G. Bentley (1983) Outline of a fear avoidance model of exaggerated pain perception, Behavior Research and Therapy, vol 21,4, 401-408.

Leventhal, H. (1979) A perceptual motor processing model of emotion. In: P. Pliner, K.R. Blankstein, \& I.M. Spigel (Eds.) Advances in the study of communication and affect, vol 5., Perception of emotions in self and others. New York: Plenum.

Leventhal, H., Brown, D., Sacham, S. \& Enquist G. (1979) Effects of preparatory information about sensations, threat of pain and attention in cold pressor distress. Journal of Personality and Social Psychology, 37, 688-714.

Leventhal, H. \& Everhart, D. (1979) Emotion, pain, and physical illness. In: C.E. Izard (Ed.) Emotions in personality and psychopathology. New York: Plenum.

Liddell, A., Bilsbury, C.D. \& Rattenbury, C. (1987) Concordance and discordance of cognitive, behavioural and somatic self-ratings as a function of exposure: A DISCAN Analysis. Behaviour Research and Therapy, 25, 5, 425-428.

Lindequist, S., Lundberg, B., Wikmark, R., Bergstad, B., LoöF, G. \& Ottermark, A. (1984) Information and regime at low back pain. Scandinavian Journal of Rehabilitation Medicine, 16, 113-116.

Linton, S.J. (1985) The relationship between activity and dnronic back pain. Pain, 21, 289294.

Linton, S.J. (1986) Behavioral remediation of chronic pain: A status report. Pain, 24, $125-141$.

Linton, S.J. (1987) Chronic Pain: The case for prevention. Behavioural Research and Therapy, 25, 4, 313-317.

Linton, S.J. \&elin, L. (1983) Applied relaxation in the management of chronic pain. Behavioural Psychotherapy, 11, 337-350.

Linton, S.]. \& Götestam, K.G. (1984) A controlled study of the effects of applied relaxation and applied relaxation plus operant procedures in the regulation of chronic pain. British Journal of Clinical Psychology, 23, 291-299.

Lintion, S.J., Melin, L. \& Stjernlöf, K. (1985) The effects of applied relaxation and 
operant activity training on chronic pain. Behavioral Psychotherapy, 13, 87-100. Loeser J. (1986) Pain and its management: an overview. In: National Irustitutes of Health Consensus Development Conference, Integrated Approach to Management of Pain, 17-19, Bethesda, MD.

Loeser, J.D. \& Fordyce, W.E. (1983) Chronic pain. In: J.E. Carr \& H.A. Dengerik (Eds.) Behavioral science in the practice of medicine. Amsterdam: Elsevier.

Love, A.W. (1987) Depression in chronic low back pain patients: Diagnostic efficiency of three self-report questionnaires. Journal of Clinical Psychology. vol 43, 1, 8489.

Love, A.W. \& Peck, C.L. (1987) The MMPI and psychological factors in chronic low back pain: A review. Pain, 28, 1-12.

Luteijn, F. Kok, A.R., van der Ploeg, F.A.E. (1980) NVM, Nederlandse Verkarte MMPI. Handleiding. Lisse: Swets \& Zeitlinger.

Luteijn, F., Starren, J. \& van Dijk, H. (1985) Handleiding bij de NPV, herziene uitgave. Lisse: Swets \& Zeitlinger.

Malec, J., Glasgow, R.E., Ely, R. \& King, J. (1977) Coping with pain: A sell-management approach. JSAS Catalog of selected Documents in PSychology, 7, 113 (ms. No. 1601).

Malone, M.D. \& Strube, M.J. (1988) Meta-analysis of non-medical treatments for chronic pain. Pain, 34, 321-244.

Maltzman, I. (1987) A neo-pavlovian interpretation of the OR and classical condotioning in humans: With comments on alcoholism and the poverty of cognitive psychology. In: G. Davey (Ed.) Cognitive processess and pavlovian conditioning in humans. Chichester: Wiley \& Sons.

Marschall, H.R. (1894) Pain, Pleasure, and Aesthetics. London: MacMillan.

Matthews, A.M. (1971) Psychophysiological approaches to the investigation of desensitization and related procedures. Psychological Bulletin, vol 76, 2, 73-91.

Mayer T.G., Tencer A.F, Kristoferson S., Mooney V. (1984) Use of noninvasive techniques for quantification of spinal range-of-motion in normal subjects and chronic low-back dysfunction patients. Spine 9, 588-595.

Mayer, T.G., Gatchel, R.J., Kishino, N., Keeley, J., Mayer, H., Capra, P. \& Mooney, V. (1986) A prospective short-term study of chronic low back pain patients utilizing novel objective functional measurement. Pain, 25, 53-68.

McCaul, K.D. \& Mallot, J.M. (1984) Distraction and coping with pain, Psychological Bulletin, 95, 516-533.

McNab, I. (1977) Backache. Baltimore: Williams \& Wilkins.

Meichembaum, D.H. (1977) Cognitive behavior modification. New York: Plenum Press.

Meichenbaum, D. \& Cameron, R. (1983) Stress inoculation training: toward a general paradigm for training coping skills. In: D. Meichenbaum \& M. Jaremko (Eds.) Stress reductiow and prevention. New York: Plenum.

Melamed, B.G. (1984) Health intervention: Collaboration for health and science. In: B.L. Hammonds \& C.J. Schreier (Eds.) Psychology and Health. Washington DC: American Psychological Association.

Melzack, R. \& Wall, P.D. (1965) Pain mechanisms: a new theory. Science, 150, 978.

Melzack, R. (1985) The McGill Pain Questionnaire, major properties and scoring methods. Pain, 1, 277-299.

Miller, N.E. (1969) Learning of visceral and glandular responses. Science, 163,434444.

Million, R., Haavik Nilsen, K., Jayson, M.I.V. \& Baker, R.D. (1981) Evaluation of low back pain and assessment of lumbar corsets with and without back sup- 
ports. Annals of the Rheumatic Diseases 40,449-454.

Mischel, W. (1968) Personality and assessment. New York: Wiley.

Moore, J.E., Armentrout, D.P., Parker, J.C. \& Kivlahan, D.R. (1986). Empirically derived pain-patient MMPI subgroups: Prediction of treatment outcome. Joumal of Behavioural Medicine, 9, 51-63.

Murphy, K.A. \& Cornish, R.D. (1984). Prediction of chronicity in acute low back pain. Archives of Physical and Medical Rehabilitation, 65, 334-337.

Nachemson, A.L. (1979) A critical look at the treatment for low back pain. Scandinavian Journal of Rehabilitation Medicine, 11, 143-149.

Naliboff, B.N, Cohen, MJ., Swanson, G.A., Bonebakker, A.D. \& McArthur D.L. (1985) Comprehensive assessment of chronic low back pain patients and controls: physical abilities, level of activity, psychological adjustment and pain perception. Pain 23, 121-134.

Nelson, R.O. \& Hayes, S.C. (1979) Some current dimensions of behavioral assessment. Behavioral assessment, 1, 1-16.

Nelson, R.O. \& Hayes, S.C. (1986) The nature of Behavioral assessment. In: R.O. Nelson \& S.C. Hayes (Eds.) Conceptual foundations of behavioral assessment. New York: Guilford Press.

Nisbett, R.E. Wilson, T.D. (1977) Telling more than we can know: Verbal reports on mental processes. Psychological Review, 84, 231-259.

Nouwen, A. (1983) EMG biofeedback used to reduce standing levels of paraspinal muscle tension in chronic low back pain. Pain, 17, 353-360.

Nuttin, J. \& Beuten, B. (1963) Handleiding bij de Persaonlijkheidsinventaris MMPI. Leuven: Leuvense Universitaire Uitgaven.

O'Leary, A. (1985) Self-efficacy and health. Behaviour Research and Therapy, 23, 437.

Öhman, A., Frederikson, M. \& Hugdahl, K. (1978) Towards an experimental model for simple phobic reactions. Behavior Analysis and Modification, 2, 97-114.

Öhman, A. (1987) The psychophysiology of emotion: an evolutionary-cognitive perspective. Advances in Psychophysiology, 2, 79-127.

Olst van, E.H., Kok, A., Orlebeke, J.F. (1980) Inleiding tot de psychofysiologie. Deventer: Van Loghum Slaterus.

Oosterbos, C.J.M., Reulings, P.G.J., Vlaeyen, J.W.S., Gerards, F., Schuerman, J.A., van Eek, H. \& Snijders, A.M.J. (1991) Changes in medical consumption and doctor shopping after interdisciplinary pain management program for CLBP patients. Manuscript in prepartion.

Pavlov, I.P. (1927) Conditioned reflexes. London: Oxford University Press.

Pearce, S. (1983) A review of cognitive-behavioral methods for the treatment of chronic pain. Journal of Psychosomatic Research, 5, 431-440.

Petrie, K. \& Azariah, R. (1990) Health promoting variables as predictors of response to a brief pain management program. The Clinical joumal of Pain, vol $6,1,43-46$.

Philips, H.C. (1980) Recent developments in tension headache research: implications for understanding and management of the disorder. In: S. Rachan (Ed.) Contributions to Medical Psychology, vol 2, Oxford: Pergamon Press.

Philips, H.C., (1987) Avoidance behaviour and its role in sustaining chronic pain. Bettaviour Research and Therapy, 25, 273-279.

Philips, H.C. (1989) Thoughts provoked by pain. Behavior Research and Therapy, 27, 4, 469-473.

Phillips, H.C. (1990) Personal Communication.

Philips, H.C. \& Jahanshahi, M. (1985) Chronic pain: an experimental analysis of the 
effects of exposure. Beh. Res. Ther $, 23,281-290$.

Philips, H.C. \& Jahanshahi, M. (1986) The components of pain behaviour report. Behavior Research and Therapy, 24, 2, 117-125.

Pinsky, J. \& Crue, D.L. (1984) Intensive group psychotherapy. In: P.D. Wall \& R. Melzack (Eds.) Textbook on pain. Edinburgh: Churchill Livingston.

Plutchik, R. (1985) On emotion: The chicken-and egg problem revisited. Motivation and Emotion, voll 9, 2, 197-200.

Rachman, S. (1977) The conditioning of fear-acquisition: A critical examination. Behaviout Research and Therapy, 15, 375-387.

Rachman, S. (1978) Human fears: A three Systems Analysis. Scandinavian Journal of Behaviour Therapy, 7, 237-245.

Rachman, S. (1981) The primacy of affect: some theoretical implications. Behaviour Research and Therapy, 19, 279-290.

Rachman, S. \& Hodgson, R. (1984) Synchrony and desynchrony in fear and avoidance. Behaviour Research and Therapy, 12, 311-318.

Redd, W.H. \& Rusch, F.R. (1985) Behavioral analysis in Behavioral medicine. Behavior Modification, 9, 131-154.

Reulings, P.G.J (1990) Vergelijking van de Pijn Cognitie Lijst met andere internationale lijsten. Unpublished report. Maastricht: University of Limburg.

Reynolds, G.S. (1968) A primer of operant conditioning. Glenview, Ill.: Scott, Foresman. Richards, J.S., Nepomuceno, C., Riles, M. \& Suer, Z. (1982) Assessing pain behavior: the UAB pain behavior scalle. Pain, 12, 393-398.

Roberts, A.H. (1986) The operant approach to the management of pain and excess disability. In: A.D. Holzman \& D.C. Turk (Eds.) Pain Management, a handbook of psychological treatment approaches. New York: Pergamon Press.

Romano, J.M. \& Turner, J.A. (1985) Chronic pain and depression: Does the evidence support a rellationship? Psychological Bulletin, vol 97, 1, 18-34.

Romano, J.M., Syrjala, K.L., Levy, R.L., Turner, J.A., Evans P. \& Keefe, F.J. (1988). Overt pain behaviors: Relationship to patient functioning and treatment outcome. Behavior therapy, 19, 191-201.

Rosenstiel, A.K. \& Keefe, F.J. (1983) The use of coping strategies in chronic low back pain patients: Relationship to patient characteristics and current adjustment. Pain, 17, 38-44.

Roskies, E. \& Lazarus, R.S. (1980) Coping theory and the teaching of coping skills. In: Davidson P.O. \& S.M. Davidson Behavioral Medicine: Changing Health Lifestyles, 38-69, New York: Brunner-Mazel.

Rudy, T.E., Turk, D.C., Brena, S.F., Stieg, R.L. \& Brody, M.C. (1990) Quantification of biomedical findings of chronic pain patients: development of an index of pathology. Pain, 42, 167-182.

Rybstein-Blinchik, E. (1979) Effects of different cognitive strategies on chronic pain experience. Journal of Behavioral Medicine, 2, 93-101.

Salter, A. (1949) Conditioning Reflex Therapy. New York: Capricorn.

Sanders, S.H. (1979) Behavior assessment and treatment of chronic pain: Appraisal and current status. In: M. Hersen, R.M. Eisler \& P.M. Miller (Eds.) Progress in behavior modification, vol 8, 249-291, New York: Academic Press.

Sanders, S.H. (1983) Automated versus self-monitoring of 'up-time' in chronic low back pain patients; a comparison study. Pain, 15, 399-406.

Sanders, S.H. (1983) Component analysis of a behavioral treatment program for chronic low back-pain. Behavior Therapy, 14, 697-705. 
Sarno, J.E. (1978) Therapeutic exercise for backpain. In: J.V. Basmajian (Ed.) Therapeutic exercise, Ballimore, MD. Williams \& Wilkins.

Savitz, D. (1985) Medical evaluation of the chronic pain patient. In: G.M. Aronoff (Ed.) Evaluation and Treatment of Chronic Pain. Baltimore: Urban \& Schwarzenberg.

Schmiat, A.I.M. (1985) Cognitive factors in the performance level of chronic low back pain patients. I. Psychosom. Res., 19, 183-189.

Schmidt, A.J.M. (1986) Persistence behavior of chronic low back pain patients. A medical psychological study. Doctoral dissertation. Maastricht: University of Limburg.

Schmidt, A.J.M. (1986) The behavioral management of pain: a criticism of a response. Pain, 30, 285-291.

Schreurs, P.J.G., Tellegen, B. \& van de Willige, G. (1984) Health, stress and coping: The development of the Utrecht Coping List. Gedrag. Tijdschrift voor Psychologie, $12,101-117$.

Schroeder H.E. \& Rich, A.R. (1976) The process of fear reduction through systematic desesitization. Journal of Consulting and Clinical Psychology, vol 44, 2, 191-199.

Schuerman, J.A. (1977) Revalidatie van gedragsdeviaties bij cerebraal gestoorden. Doctoral dissertation. Maastricht: University of Limburg.

Schultz, J.H. (1966) Das Autogene Training: Konzentrative Selbstentspanmung. Stuttgart: Georg Thieme.

Shaffer, J.W. (1979) On the analysis of rerpeated measures over time in medical, pharmacological, and behavioral research. Journal of Behavioral Medicine, vol 2, $3,221-238$.

Sherrington, (1906) Integrative Action of the Nervous System. New York: Scribner.

Shine, L.C., Bower, S.M. (1971) A one-way analysis of variance for single-subject designs. Educational and Psychological Measurement, 31, 103-113.

Simons, D.G. (1976) Muscle pain syndromes: Part 2. American Journal of Physical Medicine, 55, 15-42.

Skinner, B.F. (1953) Science and human behavior. New York: MacMillan.

Smith, T.W., Aberger, E.W., Follick, M.J. \& Ahern, D.K. (1986) Cognitive distortion and psychological distress in chronic low back pain. I. Cons. Clin. Psych., 4,573575.

Spinhoven, Ph. (1989) Hypnosis and Pain Control. Doctoral Dissertation. Leiden: University of Leiden.

SPSS Inc. (1988) SPSS/PC+V2.0 Advanced Statistics Manual, Postbus 115, 4200 AC Gorinchem.

Sternbach, R.A. (1974) Pain Patients, Traits and Treatment. New York: Academic Press.

Sternberger, L.G. \& Burns, L. (1990) Maudsley Obsessional Compulsive Inventory: obsessions and compulsions in a nonclinical sample. Behaviour Research and Therapy, 28, 4, 337-340.

Stoll, D.J. (1985) Stress Inoculation Training in the Treatment of chronic, intractable benign Pain. Michigan: University Microfilms International.

Strong, C.A. (1895) The psychology of pain. Psychology Review, 2, 329-347.

Swanson, D. W. (1984) Chronic pain as a third pathologic emotion. American Journal of Psychiatry, 141, 210-214.

Syrjala, K.L. \& Chapman, C.R. (1984) Measurement of clinical pain: a review and integration of research findings. In: C. Benedetti et al. (Eds.) Advances in Pain Research and Therapy, vol 7, New York: Raven Press.

Szasz, T.S. (1957) Pain and Pleasure: A study of Bodily feelings. New York: Basic Books. Tan, S-Y. (1982) Cognitive and cognitive-behavioral methods for pain control: $A$ 
selective review. $\mathrm{Pain}_{f} 12,201-228$.

Taylor, S.E. \& Fiske, S.T. (1978) Salience, attention and attribution: Top of the head phenomena. In: Berkowit, L. (Ed.) Advances in experimental social psychology. New York: Academic Press.

Teske, K., Daut, R.L. \& Cleeland, C.S. (1983) Relationships between nurses" observations and patiënt's self-reports of pain. Pain, 16, 289-296.

Thorndike, E.L. (1932) The findamentals of learming. New York: Teachers College.

Toomey, T., Ghia, J., Mao, W., \& Gregg, J. (1977) Acupuncture and chronic pain mechanisms: the moderating effects of affect, personality and stress on response to treatment. Pain, 3, 137-145.

Tosi, D.J. \& Baisden, B. (1984) Cognitive experiential therapy and hypnosis. In: W. Wester \& J. Smith (Eds.) Clinical Hypnosis: A Multidisciplinary Approach. New York: Lippincott.

Tucker, (1981) Lateral brain function, Emotion, and conceptualization. Psychological Bulletin, vol 89, 1, 19-46.

Turk, D.C., Meichembaum, D. \& Genest, M. (1983) Pain and Behavioral Medicine, a Cognitive-Behavioral Perspective. New York: Guilford Press.

Turk, D.C., Wack, J.T., \& Kerns, R.D. (1985) An empirical examination of the 'painbehavior' construct. Journal of Behavioral Medicine, 8, 119-130.

Turk, D.C. \& Rudy, T.E. (1986) Assessment of cognitive factors in chronic pain: a worthwhile enterprise? Journal of Consulting and Clinical Psychology, 6, 760-768.

Turk, D.C. \& Flor, H. (1987) Pain>pain behaviors: The utility and limitations of the pain behavior construct. Pain $31,277-295$.

Turk, D.C., Rudy, T.E. \& Stieg, R.L. (1988) The disability determination dilemma: toward a multiaxial solution. Pain, 34, 217-229.

Turkat, I.D., Guise, B.J. \& Carter, K.M. (1983) The effects of vicarious experience on. pain termination and work avoidance: A reflication. Behaviour Research and Therapy, 21, 491-493.

Turner, J.A. \& Chapman, C.R. (1982) Psychological interventions for chronic pain: a Critical Review II. Operant Conditioning, Hypnosis, and Cognitive-Behavioral Therapy. Pain, 12, 23-46.

Turner, J.A. \& Romano, J.A. (1984) Evaluating psychologic interventions for chronic pain: issues and recent developments. In: Benedetti et al. (Eds), Advances in Pain Research and Therapy, New York: Raven Press.

Turner, J.A. \& Clancy, S. (1988) Comparison of operant behavioral and cognitive behavioral group treatment for chromic low back pain. Journal of Consulting and Clinical Psychology, 56, 261-266.

Udolf, J.D. (1981) Handbook of Hypnosis for Professionals, New York: Van Nostrand Reinhold.

Vällfors, B. (1985) Acute, subacute and chronic low back pain: clinical symptoms, absenteism and working environment. Scandinavian Journal of Rehabilitation Medicine, supplement nr. 11.

Van den Bergh, O. \& Eelen, P. (1984) Unconscious processing and emotions. In: M.A. Reda \& M.J. Mahoney (Eds.) Cognitive psychotherapies, recent developments in theory, research, and practice. Cambridge, Ma: Ballinger.

Van Egeren, L.F. (1971) Psychophysiological aspects of systematic desensitization: some outstanding issues. Behaviour Research and Therapy, 9, 65-77.

Van Houdenhove, B., Stans, L. \& Verstraeten, D. (1987) Is there a link between "pain-proneness' and 'action-proneness"? Pain, 29, 1, 113-118. 
Vermilyea, I.A., Boice, R., Barlow, D.H. (1984) Rachman and Hodgson (1974) a decade later: how do desynchronous response systems relate to the treatment of agoraphobia? Behaviour Research and Therapy, 22, 6, 615-621.

Vitaliano, P., Russo, J., Carr, J., Maiuro, R. \& Becker, J.(1985) The ways of coping checklist: revision and psychometric properties. Multivariate Behav. Res., 20, 326.

Vlaeyen, J. (1985) De operante behandeling van chronische pijn. Tijdschrift voor klinische psychologie, $15,24-40$.

Vla eyen, J.W.S. (1990) Reply to Yanagida. Letter to the Editor. The Clinical Journal of Pain, 6, 162-163.

Vlaeyen, I.W.S. van Eek, H., Groenman, N.H. \& Schuerman, J.A. (1985) Construction of an observation scale for the behavior of pain patients. Paper presented at the 2 nd European Conference on Research in Rehabilitation, Düsseldorf, 1819 november.

Vlaeyen, J.W.S., van Eek, H., Schuerman, J.A., \& Groenman, N.H. (1987) Dimensions and components of observed chronic pain behavior. Pain, 31, 65-75.

Vlaeyen, I.W.S., Groenman, N.H. \& Legrelle, T. (1987) Traitement multimodal et interdisciplinaire de la douleur chronique. Revue de Modification du Comportement, $17,85-113$.

Vlaeyen, J.W.S., Snijders, A.M.J., Schuerman, J.A., van Eek, H., Groenman N.H. \& Bremer, J.J.C.B. (1989) Chronic pain and the three-systems model of emotions. A critical examination. CRC Critical Reviews in Physical and Rehabilitation Medicine, 2, 67-76.

Wadell, G. (1987) A new clinical model for the treatment of low back pain. Spine, 7, 632-644.

Wallston, K.A., Wallston, B.S. \& DeVellis, R. (1978) Development of the multidimensional health locus of control (MHLC) scales. Health Educ. Monogr., 6, 161 170.

Warwick, H.M.C. \& Salkovskis, P.M. (1990) Hypochondrias. Behaviour Research and Therapy, 28, 105-117.

Weiner, B. \& Graham, S. (1984) Attributional approach to emotional development. In: C.E. Izard, J. Kagan \& R.B. Zajonc (Eds) Emotionts, Cognition and Behavior. London: Cambridge University Press.

Weisemberg, M. (1987) Psychological intervention for the control of pain. Behavioural Research and Therapy, 4, 301-312.

Welsh, G.S. (1965) MMPI profiles and factor scales A and R. Journal of Clivical Psychology, 21, 43-47.

White, B. \& Sanders, S. (1985) Differential effects on pain and mood in chronic pain patients with time versus pain-contingent medication delivery. Behavior Therapy, 16, 28-35.

Wilson, G.T. (1978) The importance of being theoretical: A commentary on Bandura's "Self-efficacy: Toward a unifying theory of behavioral change". Advances in Behaviour Research and Therapy, 1,217-230.

Wilson, P.R. (1990) Chronic Pain: Medical and Bureaucratic dilemmas [Editorial]. The Clinical Jowrnal of Pain, vol 6, 2, 85-87.

Winston, A.S. B Baker, J.E. (1985) Behavior analytic studies of creativity. A critical review. The Behavior Analyst, 8, 191-205.

Wolf, S., Nacht, M. \& Kelly, J.L. (1982) EMG feedback training during dynamic movement for low back pain patients. Behavior Therapy, 13, 395-406. 
Wolpe, J. \& Lang, P.J. (1964) A fear schedule for use in behavior therapy. Behawiour Research and Therapy, 2, 27-30.

Wolpe J. \& Lang, P.J. (1977) Manual for the Fear Survey Schedule. San Diego: Educational and Industrial Testing Service.

Woods, P.J. (1985) Learning paradigms, expectancies, and behavioral control: an expanded classification for learned behavior. British Journal of Cognitive Psychotherapy, 3, 43-58.

World Health Organization (1980) International classification of impairments, disabilities, and handicaps. Geneva.

Yanagida, H. (1990) What is chronic pain? The Clinical Journal of Pain, 6, 160-161.

Zajonc, R.B. (1980) Feeling and thinking: Preferences need no inferences. American Psychologist, 35, 151-175.

Zajonc, R.B. (1984) On the primacy of affect. American Psychologist, 39, 117-123.

Zevon, M.A. \& Tellegen, A. (1982) The structure of mood change: An idiographic/ nomothetic analysis. Journal of Personality and social psychology, vol 43, 1, 111122.

Zitman, F.G., Griez, E.J.L. \& Hooijer, Chr. (1989) Standaardisering Depressievragenlijsten. Tijdschrift woor Psychialrie, 31, 114-135. 



\section{List of frequently used abbreviations}

$\begin{array}{ll}\text { ANOVA } & \text { Analysis of Variance } \\ \text { ATAPB } & \text { Audiovisual Taxonomy for Assessing Pain Behavior } \\ \text { BDI } & \text { Beck Depression Inventory } \\ \text { C } & \text { Consequence(s) } \\ \text { CHI2 } & \text { Non-parametrical CHI square statistical test } \\ \text { CHIP } & \text { Checklist for Interpersonal Pain Behavior } \\ \text { CHIP-D } & \text { CHIP-Depressive behavior } \\ \text { CHIP-DM } & \text { CHIP-Distorted Mobility } \\ \text { CHIP-N } & \text { CHIP-Nervousness } \\ \text { CHIP-NC } & \text { CHIP-Non-verbal Complaints } \\ \text { CHIP-VC } & \text { CHIP-Verbal Complaints } \\ \text { CLBP } & \text { Chronic Low Back Pain } \\ \text { FSS } & \text { Fear Survey Schedule } \\ \text { FSS-AG } & \text { FSS-Agoraphobia } \\ \text { FSS-BI } & \text { FSS-fear of Body Injury, death and Ilness } \\ \text { FSS-HA } & \text { FSS-fear of Harmless Animals } \\ \text { FSS-SA } & \text { FSS-fear of Sex and Agression } \\ \text { FSS-SO } & \text { FSS-Social Anxiety } \\ \text { FSS-TOT } & \text { FSS-Total score } \\ \text { FU1 } & \text { Follow-up measurement 1 (6 months) } \\ \text { FU2 } & \text { Follow-up measurement 2 (12 months) } \\ \text { LBP } & \text { LOw Back Pain } \\ \text { MANOVA } & \text { Multivariate Analysis of Variance } \\ \text { MMPI } & \text { Minnesota Multiphasic Personality Inventory } \\ \text { MMPI-D } & \text { MMPI-Depression } \\ \text { MMPI-F-K } & \text { MMPI-F minus K score } \\ \text { MMPI-Hs } & \text { MMPI-Hypochondrias } \\ \text { MMPI-Hy } & \text { MMPI-Hysteria } \\ \text { MMPI-Sc } & \text { MMPI-Schizofrenia } \\ \text { MOCI } & \text { Maudsley Obsessional-Compulsive Inventory } \\ \text { MOCI-CH } & \text { MOCI-Checking } \\ \text { MOCI-CL } & \text { MOCI-Cleaning } \\ \text { MOCI-TOT } & \text { MOCI-Total score } \\ \text { MOSOS } & \text { Motoric Skills Observation Scale } \\ \text { MOSOS-AR } & \text { MOSOS-Arm Rowing } \\ \text { MOSOS-LR } & \text { MOSOS-Leg Rowing } \\ \text { MOSOS-REPL } & \text { MOSOS-Replacing objects } \\ \text { MOSOS-SIT } & \text { MOSOS-Sitting tolerance } \\ \text { MOSOS-STA } & \text { MOSOS-Standing tolerance } \\ \text { MOSOS-SWIM } & \text { MOSOS-Swimming score } \\ & \end{array}$




$\begin{array}{ll}\text { MOSOS-SWIMD } & \text { MOSOS-Swimming Distance } \\ \text { MOSOS-T } & \text { MOSOS-Time score } \\ \text { MOSOS-WD } & \text { MOSOS-Walking Distance } \\ \text { MPQ } & \text { MCGill Pain Questionnaire } \\ \text { MPQ-NW } & \text { MPQ-Number of Words chosen } \\ \text { MPQ-PRI } & \text { MPQ-Present Rating Intensity } \\ \text { NPV } & \text { Nederlandse Persoonlijkheids Vragenlijst } \\ & \text { (Dutch Personality Questionnaire) } \\ \text { NPV-IN } & \text { NPV-Inadequacy } \\ \text { NPV-VE } & \text { NPV-Indignity } \\ \text { NVM } & \text { Nederlandse Verkorte MMPI } \\ & \text { (Dutch Short MMPI) } \\ \text { NVM-NEG } & \text { NVM-Negativism } \\ \text { NVM-SOM } & \text { NVM-Somatization } \\ \text { O } & \text { Organism } \\ \text { OC } & \text { Operant-Cognitive } \\ \text { OP } & \text { Operant } \\ \text { PBS } & \text { Pain Behavior Scale } \\ \text { PCL } & \text { Pain Cognition List } \\ \text { PCL-AC } & \text { PCL-Acquiescence } \\ \text { PCL-CA } & \text { PCL-Catastrophizing } \\ \text { PCL-OE } & \text { PCL-Outcome-Efficiency } \\ \text { PCL-PI } & \text { PCL-Pain Impact } \\ \text { PCL-RH } & \text { PCL-Reliance on Health Care } \\ \text { PGS } & \text { Pijn Gedrag Schaal (Pain Behavior Scale) } \\ \text { PMT } & \text { Prestatie Motivatie Test (Achievement Motivation } \\ \text { PMT-F+ } & \text { Test) } \\ \text { PMT-F- } & \text { PMT-Debilitating anxiety } \\ \text { PMT-P } & \text { PMT-Fascilitating anxiety } \\ \text { POST } & \text { PMT-Performance motivation } \\ \text { PRE } & \text { POST-treatment assessment phase } \\ \text { R } & \text { PRE-treatment assessment phase } \\ \text { S } & \text { Response } \\ \text { SIG } & \text { Stimulus } \\ \text { SIG-TEN } & \text { Schaal voor Interpersoonlijk Gedrag (Scale for Inter- } \\ \text { SIG-FRE } & \text { personal Behavior) } \\ \text { VAS } & \text { SIG-Tension } \\ \text { WLC } & \text { SIG-Frequency } \\ & \text { Visual Analog Scale } \\ & \end{array}$




\section{Summary}

This study deals with assessment and treatment of the CLBP syndrome from a Behavioral Rehabilitation perspective. It is organized into four major sections. The first, introductory, section outlines the conceptual underpinnings of the behavioral rehabilitation perspective, and its application to the area of chronic pain. This perspective will provide the context in which the following sections are embedded. The second section presents a number of studies focussing on different aspects of chronic pain assessment. The third section examines the effectiveness of two inpatient interdisciplinary behavioral treatments that have been implemented in a Dutch rehabilitation center. Finally, in the last section, a general discussion is presented.

In chapter 1, some basic concepts are discussed. Arguments are presented in favor of the consideration of pain as an emotional experience. The importance of the frequently mentioned distinction between acute and chronic pain is challenged. Two historical landmarks are described that have enhanced the evolution from the traditional disease model to the behavioral or biopsychosocial model. A clinical model is presented in which the behavioral concepts and rehabilitation concepts are integrated in a single framework. Finally, typical features of the Chronic Low Back Pain (CLBP) syndrome are discussed.

Chapter 2 presents a critical examination of the applicability of threesystems model of emotions (thus far applied exclusively to fear and anxiety) to the area of chronic pain. Most pain researchers use a trimodal conceptualization of pain, viewing it as a condition involving behavioralmotoric, cognitive, and physiological events. However, no reference is made to the theoretical model und erlying this conceptualization. For each response system, the application of both the operant and/or classical conditioning paradigm is suggested. The interrelationships among the three pain response systems, translated into the concepts of discordance and desynchrony, are highlighted. Arguments are given in favor of the use of this model as a heuristic framework in chronic pain research, assessment, and treatment. Unresolved problems are discussed, such as the lack of a consensus about the conceptualization of the three response systems and appropriate measures, and the limited knowledge about the clinical significance of discord ance and desynchrony in chronic pain. Some of the clinical and research consequences, as well as suggestions for further research, are presented. 
Chapter 3 is a replication of a study done by Turk et al. (1985) but under different conditions. It is an attempt to empirically examine the dimensions and components of observable chronic pain behavior. A broader definition of pain behavior is chosen, namely in terms of the interaction between the patient and his or her direct enviromment. The results suggest that pain behavior can be characterized by 3 dimensions: withdrawal-approach, high arousal-low arousal, and visible-audible behaviors. Furthermore, chronic pain behavior seems to be composed of at least 9 components: anxiety, attention seeking, verbal pain complaints, medication use, general verbal complaints, distorted posture and mobility, fatigue, insomnia, and depressive mood. More dimensions and components were discovered than mentioned in the study by Turk and his colleagues. However, they correspond with the variety of psychosocial problems associated with the chronic pain synd rome.

Chapter 4 describes the development of the Checklist for Interpersonal Pain Behavior (CHIP), an observation scale which assesses overt pain behavior. Taken from the dimensions and components of observed chronic pain behavior, examined in chapter 3 , this list of pain behaviors is transformed into a 78 -item rating scale to be used by clinicians or researchers to quantify observed pain behavior in a clinical setting. Six studies examine the factor structure, and the psychometric properties of this behavioral observation method. In the first study, 6 internally reliable factors are derived using factor analytic techniques from a sample of 152 chronic pain patients. They are labeled as: 'distorted mobility', 'verbal complaints', 'non-verbal complaints', 'nervousness', 'depression' and 'day-sleeping'. Because of its poor internal consistency and its limited number of items, the factor 'day-sleeping' is removed from the preliminary CHIP. Study 2 and 3 respectively show that the intra- and the inter-rater reliability of the CHIP are acceptable: Study 4 and 5 show that CHIP ratings correspond fairly well with the number of pain behaviors counted by means of the Audiovisual Taxonomy for Assessing Pain Behavior (ATAPB) and the Pain Behavior Scale (PBS). On the other hand, significant negative correlations are found between the CHIP and measures of motoric health behavior. Finally, study 6 reveals that CHIP factors 'nervousness' and 'depression' correspond reasonably well with the Welsh Anxiety Scale and the Depression scale of the MMPI. After a discussion on the advantages and the limitations of this observation scale, the conclusion seems justified that the CHIP is a useful tool in pain assessment easily to be used by different disciplines in a variety of settings.

Chapter 5 investigated the reliability and the validity of the "Pijn Gedrag Schaal' (PGS), a Dutch version of the PBS. The PGS is an observation scale tapping 10 pain behaviors using 3-point rating scales. Ratings of 8 behaviors 
(verbal complaints, vocal complaints, facial grimaces, standing posture, mobillity, body language, use of visible supportive equipment, and stationary movement) can be obtained after a relatively short observation time. For each of these items excellent reliability coefficients are found. Ratings of the two other behaviors (down-time and medication use) are only possible after at least one day observation. These last items show poor reliability coefficients and they were removed from the scale. This resulted in improved internal consistency. Validity of the 8-item PGS proves to be acceptable. The PGS appears to correlate significantly with pain behaviors counted by means of the ATAPB. On the other hand, significant negative correlations are found between the PGS and measures of heal th behavior.

While chapters 3 to 5 deal with the assessment of behavioral excesses in the overt-motoric response system, chapter 6 describes a behavioral assessment procedure for residual health behavior, named the Motoric Skills Observation Scale (MOSOS). In contrast to many other comparable tests, the MOSOS is designed as a behavior avoidance test of which the instruction is based on the pain-rest contingency principle. The MOSOS consists of three parts. Part I focuses on activities concerning cyclic movements such as walking, bicycling and rowing. Part 2 contains activities that require a minimal amount of cyclic movements such as sitting $r$ standing and replacing objects. Finally, MOSOS part 3 includes swimming as a health behavior that is being encouraged and shaped in many pain rehabilitation centers. Both inter- and intra-rater reliability of MOSOS are excellent. Generally, MOSOS has a good discriminative value: healthy pain-free controls score significantly higher on MOSOS than CLBP patients. Finally, significant negative correlations with measures of pain behavior are found, and positive correlations with a measure of fear of bodily injury. A limitation of the MOSOS as a measure of health behavior is that it only assesses avoidance of motoric activities.

Chapter 7 describes the development of an assessment instrument representing a measure for the verbal-cognitive response system of chronic pain. Fifty items, each of which are assigned to one of five factors (pain impact, catastrophizing, outcome-efficacy, acquiescence, and reliance on health care) constitute the new Pain Cognition List (PCL). The PCL is developed using a Dutch back pain patient population and shows to be stable across sex and back pain diagnosis. By means of three studies, the PCL is shown to be reliable and sufficiently valid. Moreover, the PCL appears to be a promising tool in identifying pain patients, whose pain problem is mainly controlled by cognitive factors.

Chapter 8 reports on the effectiveness of an individualized shaping program for sitting and standing intolerance in a patient with CLBP which 
lasted until more than two years following a laminectomy for removal of an intradural tumor. Assessment of sitting and standing tolerance, observation of pain behaviors, and a self-report measure regarding the pain experience were carried out during baseline, treatment, posttreatment, and at 6-month follow-up. By the end of the 6-week inpatient treatment, the patient was able to stand still for 25 minutes and to sit for 15 minutes. The overall pain behavior diminished significantly. The reported pain intensity did not increase, but did not diminish either. At 6-month follow-up, only a small decline in the results was found. These findings underscore the importance of individualized behavioral programs for chronic pain patients.

Chapter 9 describes a controlled evaluation of an operant treatment alone (OP), and in combination with a cognitive treatment $(O C)$, following a constructive approach (A brief description of the operant and the cognitive treatment is provided in appendices $A$ and $B$ respectively). A waiting list control group (WLC) is included. Subjects were 50 CLBP patients who were successively admitted to the rehabilitation center. No differences are found among the groups on several biographical data and on their MMPI profiles. The outcome measures are composite scores based on principal component analysis of raw scores of the following instruments: PBS, CHIP, MOSOS, PCL, and McGill Pain Questionnaire. The analysis resulted in 7 composite dependent variables: "Health behavior A', "Pain behavior', 'Health behavior B', "Pain experience', 'Negative affect', 'Distorted cognitions', and 'Reliance on Health care'. Their pattern of intercorrelations suggests discordance between the response systems. A repeated measures design is chosen, including 10 measurements of which groups of 3 measurements are carried out before, during and after treatment, and 1 measurement at 6 month follow-up. For the OP group, a 12-month follow-up measurement is also carried out. The results show a significant increase in health behaviors, and a significant decrease in pain behaviors, negative affect, distorted cognitions, and reliance on health care. For the variable 'Pain experience" no significant changes are found. Analysis of the differences in outcome between both experimental groups reveals that the cognitive ingredient results in more significant decrease in negative affect, and a more significant increase in some of the health behaviors. Of interest is that no differential effect is found for the variable 'distorted cognitions', suggesting that cognitive changes follow improvements in motoric behaviors.

Chapter 10 presents an extension of the previous study, and examined the clinical significance of the treatment effects. Criteria for clinical significance are described, and the percentages of patients meeting these criteria are examined for pre-treatment vs. post-treatment change, posttreatment level, and follow-up levels. For MOSOS, the experimental groups 
are compared with a pain-free control group. The results indicate that there is an acceptable number of patients meeting the criteria for clinical significant outcome. However, no significant difference between the $O P$ and the $\mathrm{OC}$ group is demonstrable. Furthermore, at post-treatment and at follow-up, levels of health behavior are comparable to those of the painfree control group.

Chapter 11 is a second extension of the study described in chapter 9 , and focuses on aspects of the broad-spectrum analysis of CLBP. In this study the relationship is examined between certain emotional problems other than pain and treatment outcome. Based on Hierarchical Classes Analysis (HICLAS), a novel clustering technique, four classes of CLBP patients can be distinguished of which 2 classes could be merged together into one class. The resulting three classes are labeled as follows: 'phobic patients', 'severely distressed patients', and 'moderately distressed patients'. A majority of the patients score high on a measure of achievement motivation, and a considerable number of patients also report phobic complaints, of which fear of body injury is the most predominant. These findings are discussed in the light of the transition from acute to chronic pain. Measures representing the most predominant problem areas do not change across treatment. In regard to treatment outcome, no differences were found between the 'moderately distressed' and the 'phobic' patients. On the other hand, the 'severely distressed' class of patients show a smaller improvement in health behavior, and, as opposed to the two other classes, their gains are lost at follow-up. Implications for treatment design and implementation are discussed.

Chapter 12 examines desynchrony between the overt-motoric and the verbal-cognitive response system following the operant and operant-cognitive treatment. Correlations were calculated among pre-post difference scores for the 7 components described in chapter 9 . Inspection of patterns of significant correlations reveals that during the OP treatment only synchrony exists between pain behaviors and health behaviors, but not between these motoric behaviors and pain cognitions. For the $O C$ group, however, synchrony is found between certain health behaviors and selfreported pain experience. In the light of the results presented in chapter 9 , the conclusion can be drawn that, as opposed to the OP treatment, the cognitive treatment ingredient leads to a decrease in pain experience, but only for those patients who improve substantially in health behaviors.

In chapter 13, methodological justifications are provided for the studies described in chapters 8 to 12 . Several issues in regard to internal and external validity, and statistical analyses are discussed, and suggestions for improvement formulated. The relatively small sample sizes, and the associ- 
ated limitations in detecting subtle differences between the experimental groups, make it likely that more differential effects may exist than those reported in this dissertation.

In the final section, in part IV, the implications of the Behavioral Rehabilitation model, and the research findings are discussed in the context of the Dutch health care system. The need for prevention of CPS, outpatient pain managemen't programs, and appropriate treatment facilities for psycho-social problems other than pain in people with CPS, is underlined. Finally, a plea is made in favour of the bridging of basic pain research, clinical outcome research, and clinical practice. 


\section{Samenvatting}

In dit proefschrift wordt zowel de diagnostiek als de behandeling van het chronische lage rugpijnsynd room (CRLP) besproken wanuit het perspectief 'Behavioral Rehabilitation'. Het eerste, inleidende deel bespreekt de conceptuele onderbouwing van het 'Behavioral Rehabilitation' perspectief, met name daar waar het zijn toepassing vindt in het gebied van de chronische rugpijnklachten. Daarin komt het theoretische kader aan de orde voor de volgende drie gedeelten. Het tweede gedeelte bevat een aantal bijdragen die betrekking hebben op de diagnostiek van chronische pijnklachten. In het derde deel wordt de effectiviteit van twee interdisciplinaire behandelingsvormen onderzocht die werden geïmplementeerd in een Nederlands revalidatiecentrum. Tenslotte worden in een laatste deel een algemene discussie, conclusie en aambevelingen gepresenteerd.

In het eerste hoofdstuk worden de basisbegrippen geïntroduceerd. Argumenten worden geformuleerd die pleiten voor de benadering van chronische pijn als emotie. Het vaak onderlijnde belang van het onderscheid tussen acute en chronische pijn wordt gerelativeerd. Twee mijlpalen in de geschiedenis van het pijn-ondl erzoek komen aan de orde. Beide hebben een belangrijke bijdrage geleverd in de ontwikkeling van het denken in termen van het traditionele ziektemodel naar het geintegreerde gedragsmodel of het biopsychosociaal model. In dit hoofdstuk wordt een voorstel gedaan tot integratie van enerzijds de begrippen eigen aan de leertheorie, en anderzijds de begrippen uit de revalidatiegeneeskunde. Tenslotte wordt ingegaan op de specifieke kenmerken van het chronische lage rugpijn-syndroom.

Hoofdstuk 2 geeft een samenvatting van het drie-factorenmodel van emoties, dat tot dusver is toegepast binnen het onderzoek naar vrees en angst, en van de bruikbaarheid daarvan bij chronische pijn. Voor elk van de drie factoren wordt een conceptueel model gepresenteerd terwijl voor ieder daarvan wordt nagegaan of het operante en/of het klassieke leerparadigma van toepassing is. Ter beschrijving van de typische partieel onafhankelijke relatie tussen de drie factoren worden de begrippen 'discordantie' en 'desynchronie' ingevoerd. Argumenten worden geformuleerd die pleiten voor de toepassing van het drie-factorenmodel als een heuristisch modell ten behoeve van onderzoek, diagnostiek en behandeling van chronische pijn. Een aantal niet opgehelderde problemen worden ter sprake gebracht, zoals het gebrek aan consensus over de 3 factoren, en de 
beperkte kennis over de klinische betekenis van de discordante verhouding tussen de factoren bij chronische pijn. Suggesties voor verder onderzoek worden gepresenteerd.

Hoofdstuk 3 is een replicatie van een studie verricht door Turk et al. (1985), maar dan onder gewijzigde condities. Deze heeft tot doel op empirische wijze de dimensies en componenten van geobserveerd chronische pijngedrag te identificeren. In tegenstelling tot Turk en zijn medewerkers is gekozen voor een bredere definitie van pijngedrag, nl. de interactie tussen de pijnlijder en zijn directe omgeving. De resultaten van deze studie suggereren dat pijngedrag gekarakteriseerd kan worden door ten minste drie dimensies.' trekt zich terug vs. laat zich merken', 'agitatie vs. rust', en 'zichtbaar vs. hoorbaar gedrag". Op basis van een hiërarchische clusteranalyse werden negen clusters ontdekt die als volgt konden worden benoemd: 'angst', 'aandacht vragen', 'verbale pijnklachten', 'medicijngebruik', 'algemene verbale klachten', 'gestoorde houding en mobiliteit', 'moeheid', "slaapproblemen', en 'depressief gedrag.' In vergelijking met de bevindingen van Turk wordt 1 dimensie meer en ongeveer het dubbele aantal clusters gevonden. De resultaten geven aan dat chronische rugpijnpatiënten een pijngedrags-patroon vertonen dat uitgebreider is dan de typische voorbeelden van pijngedrag die in de literatuur zijn beschreven. Bovendien komen de gevonden componenten overeen met de klachten die vaak geassocieerd worden met het chronische pijnsyndroom.

Hoofdstuk 4 is een logische voortzetting van hoofdstuk 3 , en beschrijft de ontwikkeling van de Checklist voor Interpersoonlijk Pijngedrag (CHIP). De CHIP is een observatieschaal voor waarneembaar pijngedrag en is gebaseerd op de pijn-gedragsitems uit het vorige hoofdstuk. Deze lijst van 78 gedragsitems is omgevormd tot een checklist waarbij elk item op basis van een zorgvuldige observatie een waardering krijgt van 0 tot 4 . Zes studies onderzoeken enerzijds de factor structuur en anderzijds de psychometrische eigenschappen van de CHIP. In de eerste studie worden middels een factor-analyse, uitgevoerd op basis van observaties bij 152 chronische pijnpatiẻnten, de volgende 6 factoren gevonden: 'gestoorde beweeglijkheid en houding', 'verbale pijnklachten", 'niet-verbale klachten", 'nervositeit', 'depressiviteit', en 'slapen overdag". Omwille van de te lage interne consistentie en het beperkte aantal items dat tot de factor 'slapen overdag' behoort, is laatstgenoemde factor uit de voorlopige $\mathrm{CHIP}$ verwijderd. Studie 2 en 3 laten zien dat zowel de intra- als de inter-beoordelaarsbetrouwbaarheid van de CHIP bevredigend is. In studies 4 en 5 wordt aangetoond dat de CHIP scores een grote overeenkomst vertonen met het aantal pijnged ragingen die geteld worden aan de hand van de 'Audiovisual Taxonomy for Assessing Pain Behavior" en eveneens met de score op de Pijn Gedrag Schaal 
(PGS). Anderzijds worden, conform de verwachtingen, significante negatieve correlaties gevonden tussen de CHIP en metingen van gezond motorisch ged rag. Tenslotte toont de zesde studie aan dat de CHIP-factoren "nervositeit" en 'depressiviteit' redelijke associaties vertonen met zelfrapportage-scores voor respectievelijk angst (Welsh Anxiety Scale) en depressie (MMPI depressie schaal). Ieder van deze bevindingen ondersteunen op eigen wijze de begripsvaliditeit van de CHIP. Na de bespreking van zowel de kracht als de beperkingen van dit nieuwe meetinstrument, wordt geconcludeerd dat de CHIP een bruikbare aanwinst is in het arsenaal van pijn meetinstrumenten, en dat deze checklist bovendien gemakkelijk gehanteerd kan worden door verschillende disciplines in verschillende settings.

Hoofdstuk 5 onderzoekt de betrouwbaarheid en de validiteit van de 'Pijn Gedrag Schaal' (PGS), een Nederlandstalige versie van de Pain Behavior Scale (PBS). De PGS is een observatieschaal bestaande uit een lijst van 10 pijngedragingen waarvan de frequentie of de intensiteit van voorkomen door middel van een driepunts waarderingsschaal beoordeeld kan worden. Scoring van 8 pijnged ragingen ('verbaal pijnged rag', 'niet-verbale vocale klachten', 'pijnlijke gezichtsuitdrukkingen', 'staande houding', 'mobiliteit', 'lichaamstaal', 'gebruik van ondersteuning', en 'zitten') blijkt betrouwbaar mogelijk na een relatief korte observatieperiode waarin de patiënt een aantal motorische activiteiten uitvoert. Voor de twee overige items ('liggen wegens pijn' en 'medicatiegebruik') is een langere observatietijd nodig. Bovendien is de betrouwbaarheid van deze 2 laatste items onvoldoende. Verwijdering van deze items uit de schaal verbeterde de interne consistentie van de PGS. De begripsvaliditeit van de uiteindelijke PGS blijkt bevredigend, gezien de bevinding dat de PGS positief correlaeert met nauwkeurige tellingen van pijnged ragingen door middel van de ATAPB, en negatief correleert met een aantal metingen van gezond gedrag, in casu motorische activiteiten. Een voordeel van de PGS is de snelle scoring. Haar beperking ligt in de restrictie van het te meten begrip pijngedrag.

In tegenstelling tot hoofdstukken 3 tot en met 5 die betrekking hebben op de meting van gedragsexcessen van de overt-motorische factor van chronische pijn, beschrijft hoofdstuk 6 een methode voor de meting van residu gezond gedrag. De Motorische Vaardigheden Observatie Schaal (MOVOS) is, in tegenstelling tot min of meer vergelijkbare instrumenten, ontwikkeld als een gedragsvermijdingstest, waarvan de instructie berust op het pijn-rust contingentie principe: "U gaat zolang door met deze activiteit tot wanneer u vanwege pijn niet meer verder kunt". De MOVOS bestaat uit drie delen. In MOVOS I wordt aan de patiënt gevraagd activiteiten uit te voeren waarvoor cyclische bewegingen nodig zijn, zoals lopen, fietsen en roeien. MOVOS deel II betreft activiteiten waarvoor een minimum aan 
cyclische bewegingen nodig zijn zoals zitten, staan en het verplaatsen van objecten. MOVOS deel III omvat een aantal zwemvaardigheden. De interen intra-beoordelaarsbetrouwbaarheid van de MOVOS blijken zeer bevredigend te zijn. Voorts vertoont de MOVOS een goed discriminatief vermogen: gezonde pijn-vrije mensen scoren significant hoger dan chronische lage rugpijn patiënten. Tenslotte worden significante negatieve verbanden gevonden tussen MOVOS scores en pijngedrag, evenals tussen MOVOS scores en angst voor lichamelijk letsel. Een beperking van de MOVOS is dat deze enkel vermijding van motorische, en bijvoorbeeld niet van sociale activiteiten quantificeert.

Hoofdstuk 7 beschrijft de ontwikkeling van een vragenlijst, gericht op de quantificering van aspecten van de verbaal-cognitieve factor van pijn. De betreffende Pijn Cognitie Lijst (PCL) bestaat uit vijftig items, behorende tot een van de volgende 5 door middel van factor-analyse verkregen factoren: 'pijn impact", 'catastroferen", 'positieve verwachting", 'berusting' en 'vertrouwen op de gezond heidszorg'. De PCL is gebaseerd op een vrij heterogene populatie van chronische rugpijnpatiënten. Toch blijkt de PCL stabiel over de beide geslachten en over de verscheidene diagnose groepen. Drie studies tonen aan dat de betrouwbaarheid en de validiteit van de PCL bevredigend zijn. Wel dienen de validiteit van de factor 'positieve verwachting' en de gevoeligheid van het instrument voor behandelingsresultaat nader te worden onderzocht:

Hoofdstuk 8 betreft een single-case study waarin de effectiviteit van een geïndividualiseerd shaping programma wordt onderzocht bij een chronische lage rugpijnpatiënte met ernstige zit- en sta-problemen. Vanwege de ernst van haar klachten bracht deze 50-jarige vrouw de tijd meestal liggend of lopend door. Haar pijnklachten, ontstaan na een laminectomie ter verwijdering van een intradurale tumor, persisteerden tot 2 jaar na deze chirurgische ingreep. Tolerantiebepaling voor zitten en staan, observaties van pijngedrag en zelf-rapportage over de ervaren pijn-intensiteit werden uitgevoerd vóór, tijdens en na de behandeling, en bij 6 maanden follow-up. Na de 6 weken durende klinische behandeling was deze patiënte weer in staat om gedurende perioden van 25 minuten te staan, en 15 minuten te zitten. Het pijngedrag nam significant af. De ervaren pijn-intensiteit nam niet toe, noch verminderde deze na de behandeling. Desond anks bleven genoemde resultaten ten aanzien van de uitbreiding van haar gedragsrepertoire behouden tot 6 maanden na ontslag. Deze bevindingen bevestigen de waarde van geindividualiseerde gedragsmatige behandelingsprogramma's voor mensen met chronische pijnklachten.

Hoofdstuk 9 beschrijft een gecontroleerde studie die de effectiviteit onderzoekt van een operante behandeling (OP) en een gecombineerde 
operant-cognitieve behandeling $(\mathrm{OC})$. Een korte beschrijving van de operante en de cognitieve behandeling is opgenomen in appendices $A$ en B. Een wachtlijst controle groep (WLC) is betrokken bij het onderzoek. Subjecten zijn 50 chronische lage rugpijn patiënten die achtereenvolgens, meestal in groepjes van 4 , werden opgenomen in een revalidatiecentrum. De drie groepen vertonen grote gelijkenis wat betreft een aantal biografische variabelen en MMPI profielen. De effect variabelen bestaan uit samengestelde scores die gebaseerd zijn op een principale componenten analyse toegepast op de ruwe scores van de PGS, CHIP, MOVOS, PCL, en McGill Pain Questionnaire. Deze analyse resulteerde in de volgende 7 componenten die verder als afhankelijke variabelen worden gehanteerd: 'Gezond gedrag A', 'Pijngedrag', 'Gezond gedrag B', 'Pijnervaring', 'Negatief affect', 'Gestoorde cognities', en 'Vertrouwen op de gezondheidszorg'. Het patroon van intercorrelaties tussen deze variabelen suggereert discordantie tussen de motorische en de cognitieve factor van chronische lage rugpijn. Gekozen werd voor een design met herhaalde metingen: drie metingen vóór, tijdens en ná de behandeling, evenals een 6-maanden follow-up meting. Voor de OP groep werd bovendien een 12-maanden follow-up meting uitgevoerd. De resultaten tonen een significante toename in gezond gedrag en een significante afname in pijngedrag, negatief affect, gestoorde cognities, en overmatig vertrouwen op de gezondheidszorg. Ondanks de toename in gezond gedrag bleef de pijnervaring ongewijzigd. Analyse van de verschillen tussen de beide behandelingsvormen toont aan dat de cognitieve behandelingsingredient verantwoordelijk geacht kan worden voor een sterkere afname van negatief affect, en een sterkere toename van een aantal gezonde gedragingen. Opmerkelijk is dat geen differentieel effect is gevonden voor de afname in gestoorde cognities. Deze laatste bevinding suggereert dat cognitieve veranderingen bij pijnpatiënten wellicht het gevolg zijn van veranderingen in motorisch gedrag.

In hoofdstuk 10 wordt, in tegenstelling tot hoofdstuk 9 waarin statistisch significante verschuivingen worden geanalyseerd, de klinische significantie van de gevonden resultaten onderzocht. Criteria voor klinische significantie worden beschreven en percentages van patiënten wier resultaten overeenkomen met deze criteria onderzocht. De MOVOS-gegevens van beide experimentele groepen werden vergeleken met die van een gezonde controlegroep. De resultaten suggereren dat een bevredigend aantal patiënten aan de gestelde criteria voldoet, maar dat geen significante verschillen worden gevonden tussen beide behandelingsvormen. Opvallend is dat na de behandeling en bij follow-up het bereikte niveau van gezond gedrag vergelijkbaar is met dat van de gezonde controle groep.

Hoofdstuk 11 onderzoekt de gevolgen van de behandeling voor andere 
klachten dan pijn. Gebruik makend van de recent ontwikkelde Hiërarchische Klassen Analyse (HICLAS), kunnen vier groepen van patiënten worden onderscheiden, waarvan twee groepen bij elkaar gevoegd kunnen worden. De drie hieruit resulterende groepen zijn CLRP-patiënten met respectievelijk fobische klachten, ernstige en multipele psycho-sociale klachten, en matige psycho-sociale klachten. Opvallend is dat een grote meerderheid van de patiënten hoog scoren op de PMT-P, een maat voor prestatie-motivatie. Verder rapporteert een behoorlijk aantal patiënten fobische klachten, waarvan vrees voor lichamelijk letsel domineert. Deze bevindingen doen vragen rijzen met betrekking tot de rol die deze klachten hebben in de overgang van acute naar chronische pijn. Veder wordt aangetoond dat deze klachten niet veranderen als gevolg van de behandeling. Een opvallende bevinding met betrekking tot het therapie resultaat is dat geen verschillen worden gevonden tussen de patiènten met fobische klachten en die met matige psycho-sociale klachten. Daarentegen tonen de patiënten met ernstige en multipele psycho-sociale klachten minder vooruitgang, hoewel nog steeds significant, dan de patiënten uit de twee andere groepen. Bovendien is er in tegenstelling tot de twee andere groepen bij hen meer terugval bij follow-up. De implicaties van deze bevindingen voor toekomstige therapieplanning en opzet worden besproken.

Hoofdstuk 12 onderzoekt het desynchroon verloop tussen de overtmotorische en de cognitieve factor van pijn tijdens de operante en operantcognitieve behandeling. Correlaties worden berekend tussen pre-post verschilscores voor de 7 componenten die in hoofdstuk 9 zijn beschreven. Het patroon van significante correlaties maakt het waarschijnlijk dat de cognitieve behandelingsingrediënt een verlaging van de pijnervaring tot gevolg heeft bij revalidanten bij wie een duidelijke toename in gezond ged rag is vastgesteld.

Hoofdstuk 13 geeft een methodologische verantwoording van de studies beschreven in de hoofdstukken 8 tot en met 12. Maatregelen ten behoeve van de interne en de externe validiteit, en een aantal kanttekeningen betreffende de keuze van de statistische analyses worden besproken. De relatief kleine steekproeven, en de daarmee samenhangende beperkte power om reële verschillen te detecteren, maken het zeer waarschijnlijk dat in werkelijkheid meer differentiële effecten aanwezig zijn dan die welke in dit proefschrift zijn beschreven.

Tenslotte worden in het laatste gedeelte algemene conclusies getrokken met betrekking tot de onderzoeksbevindingen, en word de mogelijke plaats van de 'Behavioral Rehabilitation' in de Nederlandse gezondheidszorg besproken. Verder wordt de noodzaak bepleit van preventieve maatregelen, poliklinische behandelingsprogramma's en speciale voorzieningen voor 
de behandeling van psycho-sociale klachten bij mensen met het chronische pijnsyndroom. Tenslotte wordt opnieuw een lans gebroken voor de overbrugging van de kloof tussen fundamenteel en klinisch pijn-onderzoek. 


\section{Dankwoord}

Dit proefschrift is de vrucht van het werk van velen. Allereerst wil ik Joop Schuerman en Aja Bakker-Boerrigter danken. Zij hebben gezorgd voor een stevige voedingsbodem waarin het $\mathrm{CBP}$-project en uiteindelijk dit proefschrift is ontkiemd en gegroeid. Joop, met je jarenlange ervaring heb je voor mij het pad geëffend, en vele ideeën werden in de talloze discussies met jou geboren. Aja, jij hebt me steeds alle vrijheid gegeven op de afdeling en op bijzondere wijze wegwijs gemaakt in het leggen van contacten met verscheidene medische en para-medische disciplines.

Hugo van Eek en Ank Kole-Snijders, jullie zijn niet voor niets paranimfen. Hugo, jij bent de vaste trait d'union met het team 4 . Onze vriendschap is voor mij een persoonlijke verrijking. Ank, met je heldere kijk op dingen heb je met mij de grote lijnen bewaakt.

Joost Bremer, als promotor stimuleerde je mij van den beginne om mijn ideeën aan te scherpen door ze aan papier toe te vertrouwen. Mijn dank voor de nauwgezetheid waarmee je de vele concepten hebt weten bij te sturen tot leesbare stukken. Nico Groenman, ambassad eur van de operante pijnbehandeling in Nederland, je hebt het onderzoek een goeie start gegeven. Op de statisch-methodologische deskundigheid van Jan van Houtem heb ik verscheidene malen beroep gedaan. Dank voor je geduld en continue inzet. Wout Dingemans en Godfried Saes, jullie medische ondersteuning was onontbeerlijk. Ook Paul de Boeck, Luc Goossens en Paul Eelen uit Leuven, alsook de leden van de Vlaamse inter-universitaire werkgroep pijn-psychologen wil ik van harte danken voor de waardevolle tips en inspirerende discussies.

Nog tientallen anderen waren onmisbaar. Redmond Reams, thanks for your careful reading and suggestions. Syl Geurts en Eric Vermetten, de PCL-E blijft met jullie verbonden. Jan Thomassen, Rob Pelt, Rob Coenen, Carla en Guido Hoogervorst, Henk Prins, Jos Offermans, Marlies Wroniak, Christa Busink, Cathy Heltzel, Noël Dortu, Herman Mulder, Annelies Willen, Yvonne Jegers, Rob Smeets, de verpleegkundigen en arts-assistenten van team 4 van het $\mathrm{RCH}$, jullie waren bereid om het therapeutisch handelen af te stemmen op het onderzoek. Dank voor het vertrouwen. Tiny FasottiDumont, Carol Magnée, Fon van Knippenberg, Ben Noorloos, Dia Pernot, en Mieke Witte, zond er jullie waren er gewoonweg geen data. Marlie Hahn presteerde het om onder grote tijdsdruk toch weer zoveel vellen typewerk 
af te leveren. Ben Eisermann, je bent een meester in het maken van figuren. Dank aan jullie allen.

Tenslotte een extra woord van dank voor de revalidanten, die bereid willig meewerkten aan die vele en lange meetdagen. 


\section{Curriculum vitae}

De auteur van dit proefschrift werd geboren op 26 september 1957 te Brussel. Na het behalen van het diploma middelbaar onderwijs, richting Wetenschappelijke-A aan het H.- Hart College te Wezembeek-Oppem (België), studeerde hij Psychologische Wetenschappen aan de Vrije Universiteit te Brussel. In 1980 voltooide hij zijn studie Psychologie in de richting Klinische Psychologie. In het kader van zijn burgerdienst was hij gedurende 15 maanden verbonden aan de afdeling gedragstherapie van het Universitair Psychiatrisch Centrum St.-Jozef te Kortenberg (Prof. dir. P. Cosyns). Na enkele maanden als vrij assistent verbonden te zijn geweest aan de Katholieke Universiteit te Leuven werd hem een studiebeurs aangeboden door de Belgian American Educational Foundation, die hem in 1982 in de gelegenheid stelde een klinische opleiding te volgen aan de University of Washington te Seattle (Prof. W.E. Fordyce, PhD). In 1983 behaalde hij het APA-approved Psychology Internship Certificate. Na een tijdelijke functie aan de Université Catholique de Louvain, het Psychosociaal Centrum St. Alexius, en de Kliniek voor Multiple Sclerose te Melsbroek te Brussel, was hij vanaf 1985 verbonden aan de vakgroep Medische Psychologie van de Rijksuniversiteit Limburg te Maastricht, met detachering naar het Instituut voor Revalidatie Vraagstukken te Hoensbroek. Hij is geregistreerd klinisch psycholoog en lid van het Nederlands Instituut voor Psychologen. Verder is hij aspirant lid van de Vlaamse Vereniging voor Gedragstherapie en lid van de Vlaamse Vereniging voor Autogene Training en Hypnotherapie. 


\section{Publications (chronologically)}

Cosyns, P. \& Vlaeyen, J. (1983) La douleur chronique. In: Fontaine et al. (Eds.) Cliniques de Therapies Comportementales. Liège: Mardaga.

Cosyns, P. \& Vlaeyen, J. (1\$83) Pijn, een leertheoretische benadering Journal of Head $\mathcal{G}$ Neck Pathology, 2, 80-84.

Vlaeyen, J. \& Groenman, N.H. (1984) Chronische pijn en het operante pijnbehandelingsprogramma. In: Mattie H. et al. (Eds) Pijwinformatorium. Alphen aan den Rijn: Stafleu.

Vlaeyen, J.W.S. (1985). De operante behandeling wan chronische pijn. Tijdschrift voor Klinische Psychologie, 15, 24-40.

Vlaeyen, J.W.S. (1985). Het gebruik van de MMPI "subtle" en "obvious" subschalen bij de diagnostiek van chronische pijnpatiënten. Bulletin Klinische Praktijk van de Medische Psychologie. Erasmus Universiteit Rotterdam, jaargang 2, nr. 2.

Vlaeyen, J.W.S. (1985). Chronische pijn vanuit gedragsgeneeskundig perspectief. Readaptation-Revalidatie, Tijdschrift der Belgische seminaria voor Revalidatie, Facs. $4 / 1985$.

Groenman, N.H., J.A. Schuerman, J.W.S. Vlaeyen \& H. van Eek (1986). Chronische pijn. In: Kaptein, A.A. e.a. (Red.). Behtavioral Medicine: Psychologische behandeling wan lichamelijke aandoeninger. Alphen a/d Rijn: Samson Stafleu.

Eek, H. van, J.W.S. Vlaeyen, J.A. Schuerman, N.H. Groenman \& A.M.J. Snijders (1987). Chronische pijn: recente ontwikkelingen. Gedrag en Gezondheid, 15, 3, 99. 106.

Vlaeyen, J.W.S., H. van Eek, N.H. Groenman \& J.A. Schuerman (1987). Geobserveerd chronisch pijngedrag: dimensies en componenten. Gedragstherapie, 20, 2, 111 123.

Vlaeyen, I.W.S., N.H. Groenman \& T. Legrelle (1987). Traitement multimodal et interdisciplinaire de la douleur chronique. Revue de Modification du Comportement, $17,2,95-113$.

Vlaeyen, J.W.S., H. van Eek, N.H. Groenman \& J.A. Schuerman (1987). Dimensions and components of observed chronic pain behavior. Pain, 31, 65-75.

Vlaeyen, J.W.S., van Eek, H., Groenman, N.H. \& Schuerman, J.A. (1987). Development of an observation scale for the behavior of chronic pain patients. International Journal of Rehabilitation Research, supplement no. 5, vol 10, 4, 263.266.

Groenman, N.H., J.W.S. Vlaeyen, A. Bakker-Boerrigter, H. wan Eek, J.A. Schuerman \& A.M.J. Snijders (1988). Chronische pijn als aangeleerd gedrag. Bijblijuen cumulatief geneeskundig mascholingssysteem, themanummer Pijn, 4 (10), 18-30.

Groenman, N.H., J.W.S. Vlaeyen, H. van Eek, J.A. Schuerman \& A.M.J. Snijders (1989). Chronische Pijn. In: Breteler, M., R. Beunderman, B. Garssen \& A.A. Kaptein (Red.). Behavioral Medicine. Leiden: Boerhaave Commissie voor Postacademisch Onderwijs in de Geneeskunde.

Pelt, R.A.G.B., J.W.S. Vlaeyen, H. van Eek, J.M.C. Thomassen (1989). Fysiotherapie bij chronische benigne pijn. Nederlands Tijdschrift woor Fysiotherapie, Vol. 99, No. $6,140-142$.

Pelt, R.A.G.B., J.W.S. Vlaeyen, H. van Eek, J.M.C. Thomassen (1989). Fysiotherapy and chronic pain. Nederlands Tijdschrift voor Fysiotherapie, Vol. 99, No. 6, 173174. 
Vlaeyen, J.W.S., N.H. Groenman, J. Thomassen, J.A. Schuerman, H. van Eek, A.M.J. Snijders, J. van Houtem (1989). A Behavioral Treatment for Sitting and Standing Intolerance in a Patient with Chronic Low Back Pain. The Clinical Journal of Pain, 5: 233-237.

Vlaeyen, J.W.S., A.M.J. Snijders, J.A. Schuerman, H. van Eek, N.H. Groenman \& I. JC.B. Bremer (1989). Chronic pain and the three systems model of emotions: A critical examination. $C R C$ Critical Reviews in Physical and Rehabilitation Medicine, 1, 67-76.

Vlaeyen, J.W.S., H. van Eek, A.M.J. Snijders, N.H. Groenman \& J.A. Schuerman (1989). The treatment of chronic low back pain in rehabilitation. IRV Research Report -1988, Hoensbroek.

Vlaeyen, J.W.S. H. van Eek, A.M.J. Snijders N.H. Groenman \& J.A. Schuerman (1989). De operante behandeling van chronisch lage rugpijn: een evaluatie met herhaalde metingen. Gedragstherapie, 22, 93-105.

Vlaeyen, J.W.S. A.M.J. Snijders, J.A. Schuerman, J.J.C.B. Bremer, H. van Eek \& N.H. Groenman (1989). Chronische pijn en het drie-factorenmodel van emoties.

Een kritische beschouwing. Tijdschrift voor Psychiatrie, 31, 100-113.

Vlaeyen, J.W.S., S.M. Geurts, H. van Eek, A.M.J. Snijders, J.A. Schuerman, N.H. Groenman (1989). Pijn Cognitie Lijst, experimentele versie. Lisse: Swets \& Zeitlinger. Vlaeyen, J.W.S. \& S.M. Geurts (1989). Boekbespreking van Van Houdenhove (Red.). Mijn pijn is toch niet ingebeeld? Tijdschrift voar Psychiatrie, 31 , boekennummer 2, 84-85.

Vlaeyen, J.W.S., J.A. Schuerman, H. van Eek, N.H. Groenman \& A.M.J. Snijders (1989). Chromische benigne pijn: behandelling, meetinstrumenten, resultaten. Nederlands Tijdschrift voor Geneeskunde, 133 (3), 140-141.

Groenman, N.H., Vlaeyen, J.W.S., van Eek, H. \& Schuerman, N.H. (1990) Chronic Pain. In: A.A. Kaptein, H.M. van der Ploeg, B. Garssen, P.J.G. Schreurs \& R. Beunderman (Eds.) Behavioural Medicine, Psychological treatment of somatic disorders. Chichester: John Wiley \& Sons.

Kole-Snijders, A.M.J., J.W.S. Vlaeyen, H. van Eek, J.A. Schuerman \& N.H. Groenman (1990). Cognitie en cognitieve behandeling van chronische pijn: een bron van verwarring. Gedragstherapie, 23, 1, 3-16.

Vlaeyen, J.W.S. (1990). Boekbespreking van Van Houdenhove (Red.): Mijn pijn is toch niet ingebeeld? Gedragstherapie, 23, 3, 227-230.

Vlaeyen, J.W.S. (1990) Reply to Yanagida. Letter to the Edikor. The Climical Journal of Pain, 6, 162-163.

Vlaeyen, JW.S., Pernot, H., Snijders, A.M.I., Van Eek, H., Schuerman, J.A. \& Groenman, N.H. (1990). Betrouwbaarheid en validitteit van een nederlandse versie van de "Pain Behavior Scale". Tijdschrift voor de Psychologie en haar grensgebieden, 45, 184-189.

Vlaeyen, J.W.S., Kole-Snijders, A.M.J., van Eek, H., Groenman N.H. \& Schuerman J.A. (1990) Het chronische pijnsyndroom. Dth, Tijdschrift voor directieve Therapie en Hyprose, 10, 4, 343-358.

Vlaeyen, J.W.S., Kole-Snijders, A.M.J., van Eek, H., Groenman N.H. \& Schuerman J.A. (1990) A comment on the Schmidt/Fordyce discussion. Pain, 43, 131-132. Vlaeyen, I.W.S., S.M. Geurts, H. van Eek, A.M.J. Snijders 『.A. Schuerman \& N.H. Groenman (1990). What do chronic pain patients think of their pain: Development of a pain cognition questionnaire. British Journal of Clinical Psychology, 29, 4, 383-394. 
Vlaeyen, J.W.S. (1990). Boekbespreking van Van der Kloot \& Vertommen. De MPQDLV. Tijdschrift voor Psychiatrie, (in druk)

Vlaeyen, J.W.S., S.M. Geurts, H. van Eek, A.M.J. Snijders, J.A. Schuerman \& N.H. Groenman (1990). Attributies, zelf-effectiviteit en pijn: Ontwikkeling van een pijncognitie vragenlijst. Gedrag en Gezondheid, 18, 6, 295-299.

Vlayen, J.W.S., S.M. Geurts, H. van Eek, A.M.J. Kole-Snijders, J.A. Schuerman \& N.H. Groenman (1990). Comment la douleur chronique est-elle vécu? Development d'un questionnaire sur la cognition de la douleur. Science et comportement, 20, No. 2.

Vlaeyen, J.W.S., D. Pernot, H. van Eek, A.M.J. Snijders, J.A. Schuerman \& N.H. Groenman (1990). Assessment of the components of observed chronic pain behavior: The checklist for interpersonal pain behavior (CHIP), Pain, 43, 337347.

Vlayen, J.W.S., D. Pernot, H. van Eek, A.M.J. Snijders, J.A. Schuerman \& N.H. Groenman (1991). The checklist for interpersonal pain behavior (CHIP), in M.R. Bond, J.E. Charlton \& C.J. Woolf (Eds.). Proceedings of the VIth World Congress on Pain. Amsterdam: Elsevier Science Publishers, 531-537. 